\title{
Loreclezole : a clinical evaluation of its safety and efficacy as anticonvulsant
}

Citation for published version (APA):

Rentmeester, T. W. (1991). Loreclezole : a clinical evaluation of its safety and efficacy as anticonvulsant. [Doctoral Thesis, Maastricht University]. Rijksuniversiteit Limburg. https://doi.org/10.26481/dis.19911219tr

Document status and date:

Published: 01/01/1991

DOI:

10.26481/dis.19911219tr

Document Version:

Publisher's PDF, also known as Version of record

\section{Please check the document version of this publication:}

- A submitted manuscript is the version of the article upon submission and before peer-review. There can be important differences between the submitted version and the official published version of record.

People interested in the research are advised to contact the author for the final version of the publication, or visit the DOI to the publisher's website.

- The final author version and the galley proof are versions of the publication after peer review.

- The final published version features the final layout of the paper including the volume, issue and page numbers.

Link to publication

\footnotetext{
General rights rights.

- You may freely distribute the URL identifying the publication in the public portal. please follow below link for the End User Agreement:

www.umlib.nl/taverne-license

Take down policy

If you believe that this document breaches copyright please contact us at:

repository@maastrichtuniversity.nl

providing details and we will investigate your claim.
}

Copyright and moral rights for the publications made accessible in the public portal are retained by the authors and/or other copyright owners and it is a condition of accessing publications that users recognise and abide by the legal requirements associated with these

- Users may download and print one copy of any publication from the public portal for the purpose of private study or research.

- You may not further distribute the material or use it for any profit-making activity or commercial gain

If the publication is distributed under the terms of Article $25 \mathrm{fa}$ of the Dutch Copyright Act, indicated by the "Taverne" license above, 


\section{LORECLEZOLE}

A CLINICAL EVALUATION OF ITS SAFETY AND EFFICACY AS ANTICONVULSANT

\section{PROEFSCHRIFT}

ter verkrijging van de graad van doctor

aan de Rijksuniversiteit Limburg te Maastricht, op gezag van de Rector Magnificus, Prof. mr M.J. Cohen, volgens het besluit van het College van Dekanen, in het openbaar te verdedigen op donderdag, 19 december 1991 om 14.00 uur

door

Theodorus Willem Rentmeester geboren te Castricum 
Promotores:

Prof. dr H.A.J. Struyker Boudier

Prof. dr H. Meinardi

Beoordelingscommissie:

Prof. dr F. Spaans, voorzitter

Prof. dr P.J. Brombacher

Prof. dr C.H.M. Brunia

Prof. dr J.M.J.P. van der Linden

Prof. dr J.W. van Ree

Dr W.O. Renier

\section{CIP-DATA KONINKLIJKE BIBLIOTHEEK, DEN HAAG}

Rentmeester, Theodorus Willem

Loreclezole: a clinical evaluation of its safety and efficacy as anticonvulsant / Theodorus Willem Rentmeester. - [S.I. : \$.n.] - III.

Proefschrift Maastricht. - Met lit. opg. - Met samenvatting in het Nederlands.

ISBN 90-9004590-2

Trefw.: epilepsie / geneesmiddelen. 


\section{Contents}

List of abbreviations

1. Some aspects of epilepsy and the treatment of epilleptic seizures ........... 7

1.1. Introduction ........................................................................................... 7

1.2 Treatment of epileptic seizures …........................................................... 10

1.3 Hypotheses on the action of antiepileptic drugs ...................................... 11

1.4 The need to develop new antiepileptic drugs …....................................... 13

1.4.1 Phenobarbital ................................................................................ 14

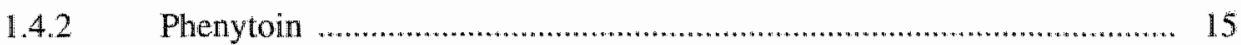

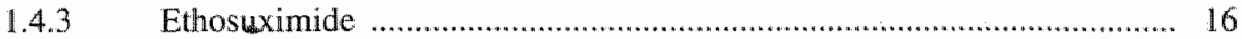

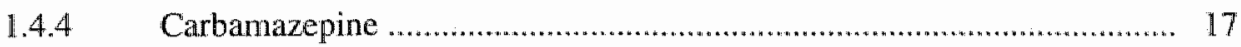

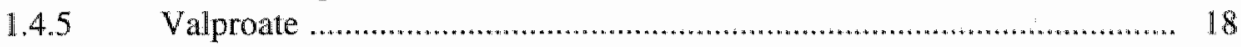

1.4.6 Benzodiazepines ...................................................................... 19

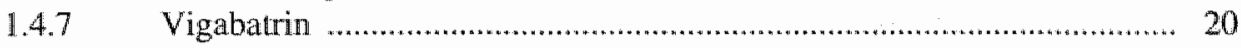

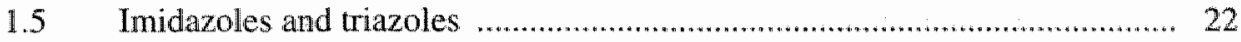

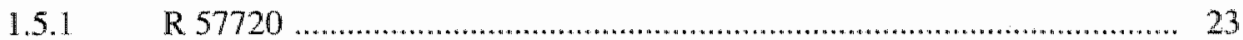

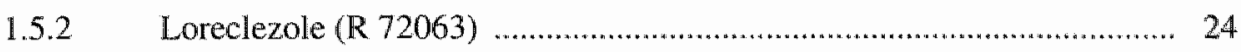

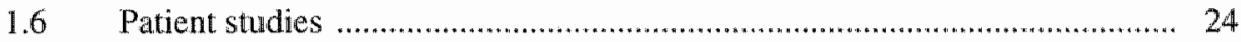

1.6.1 Various stages in patient studies ......................................................... 25

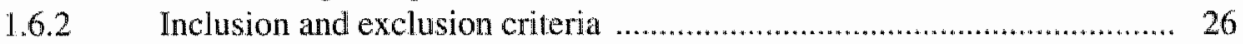

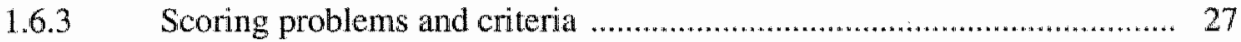

1.6.4 Ethical aspects and informed consent ................................................. 28

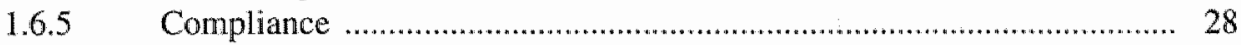

1.7 Designs for studies with loreclezole ……................................................ 29

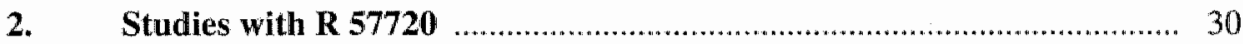

$2.1 \quad$ Volunteer studi .................................................................................. 30

$2.2 \quad$ Single dose studies in patients ............................................................. 30

2.3 Multiple dose studies with ascending doses in patients ............................. 30

2.4 Efficacy and safety evaluation of increasing dosages of R 57720

2.4.1 Methods and patients ...................................................................... 31

2.4.1.1 Inclusion and exclusion criteria ................................................. 31

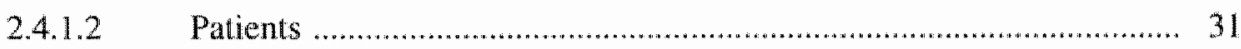

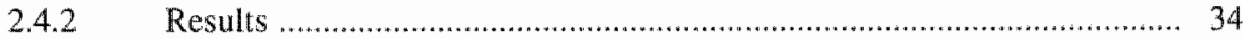

2.4.2.1 Seizure frequency ................................................................ 34

2.4.2.2 Adverse events ........................................................................... 34

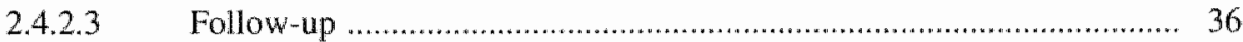

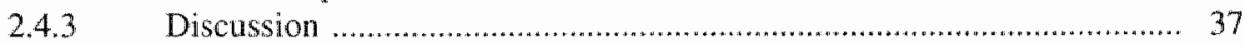

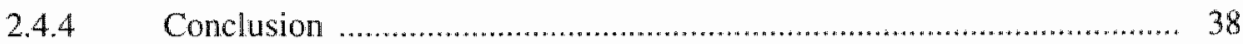

3. Open study of the efficacy and safety of loreclezole as add-on therapy in therapy resistant epilepsy patients ........................................ 39

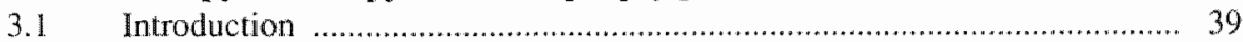

3.2 Methods and patients ......................................................................... 39

3.2.1 Inclusion and exclusion criteria ................................................. 39 


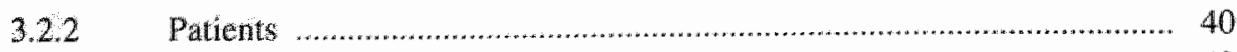

32.3. Methods of determination of Joreclezole ............................................... 43

3.2.3.1. Sample processing ....................................................................... 43

3.2.3.2. Chromatography ................................................................... 43

3.3 Results of the single dose stud .......................................................... 44

3.3.1 Plasma concentrations ....................................................................... 44

3.3.2 Adverse events ........................................................................ 45

3.4 Results of the chronic treatment study ................................................. 45

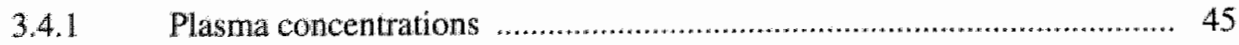

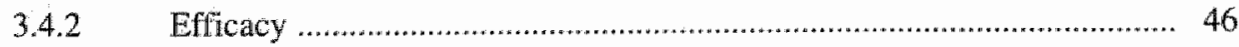

3.4.3 Adverse events .......................................................................... 48

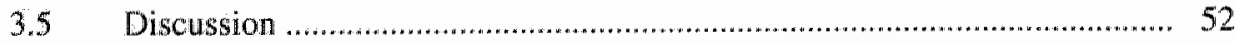

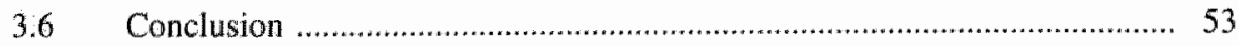

4. Evaluation of one-year treatment with loreclezole;

follow-up study of an open add-on study of the efficacy and safety of loreclezole .............................................................................................. 55

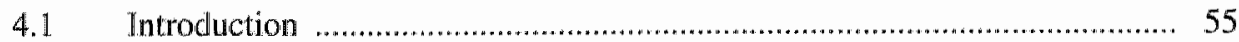

4.2 Methods and patients ...................................................................... 55

4.2.1 Inclusion and exclusion criteria ..................................................... 55

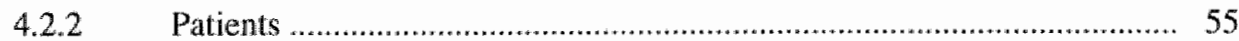

4.2.3 Seizure severity score ................................................................... 56

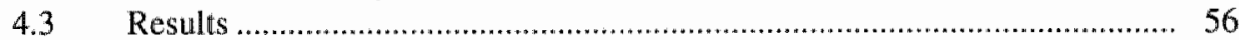

4.3.1 Plasma concentrations ..................................................................... 57

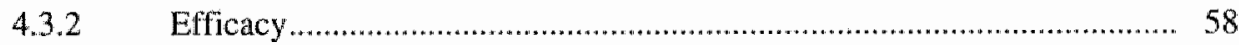

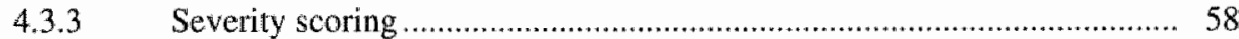

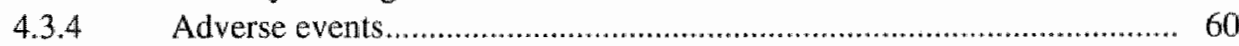

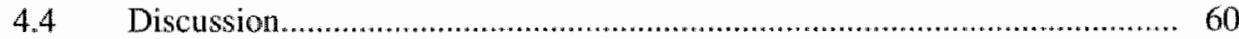

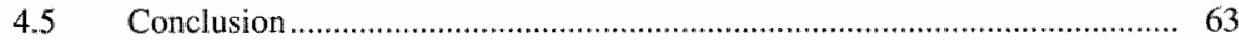

5. Double-blind placebo controlled add-on study of the efficacy and safety of loreclezole in epilepsy patients ................................................. 65

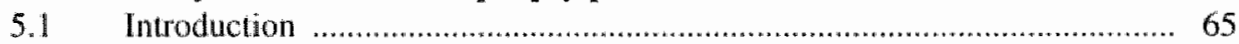

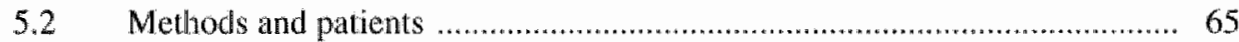

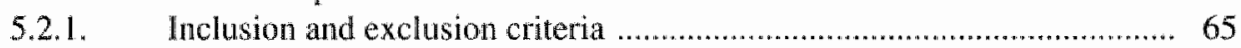

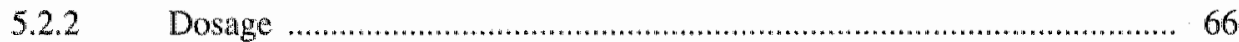

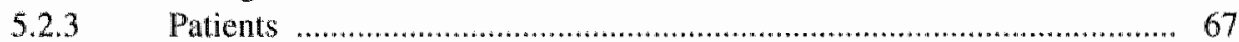

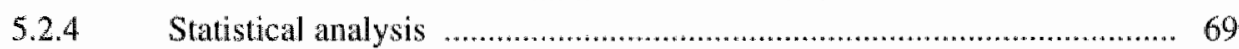

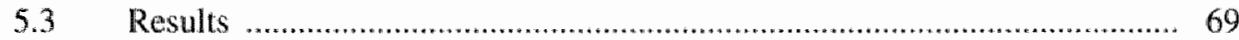

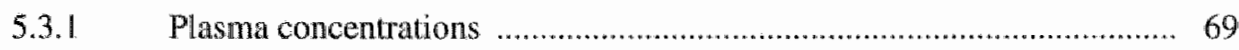

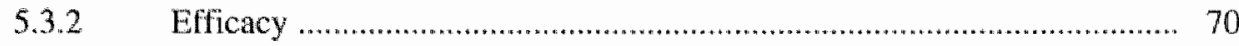

5.3.3 Adverse events ........................................................................... 71

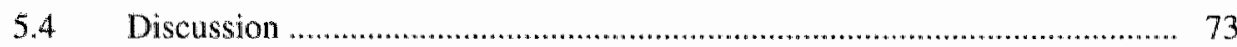

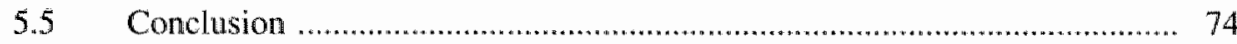


6. Open follow-up evaluation of the efficacy and safety of loreclezole as add-on therapy in patients with uncontrolled partial seizures ........... 76

6.1 Introduction .............................................................................. 76

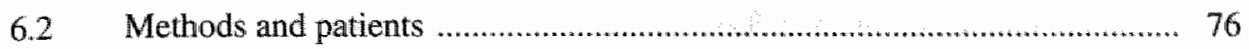

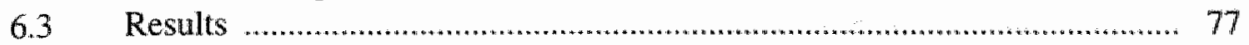

6.3.1 Plasma concentrations ……............................................................ 77

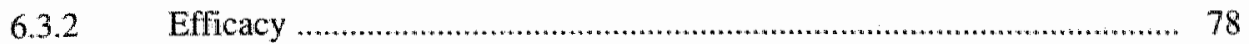

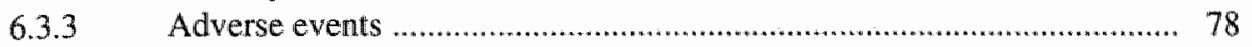

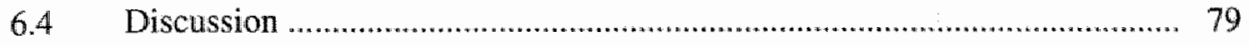

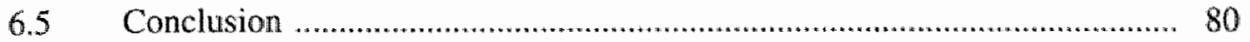

7. Gradual transition to loreclezole monotherapy .................................... 81

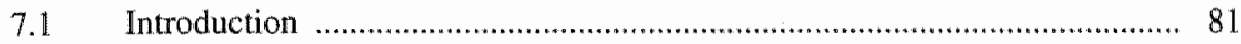

7.2 Methods and patients .............................................................................. 81

7.2.1 Inclusion and exclusion criteria ……............................................ 83

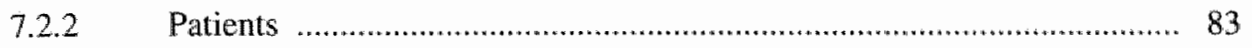

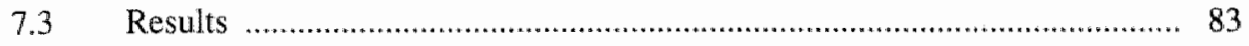

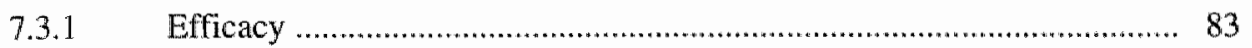

7.3.2 Plasma concentrations ………….................................................... 84

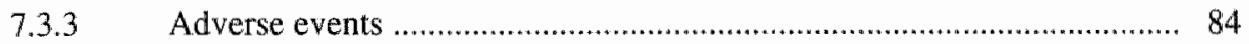

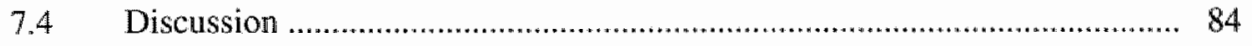

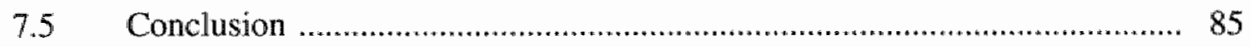

8. Evaluation of the efficacy and safety of loreclezole ............................. 86

8.1 Introduction …................................................................................. 86

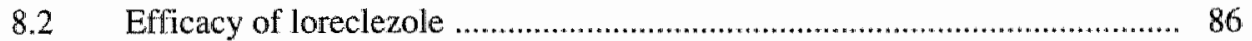

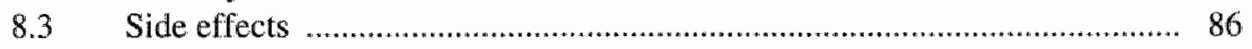

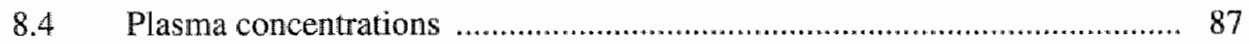

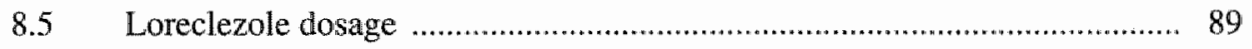

$8.6 \quad$ Methods of investigation ..................................................................... 91

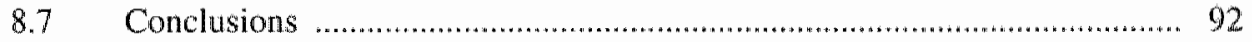

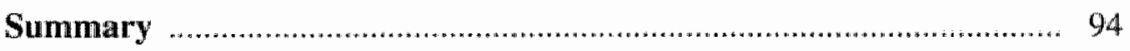

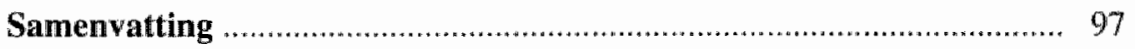

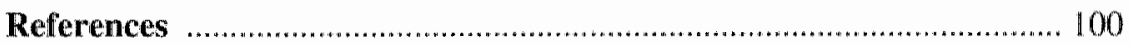

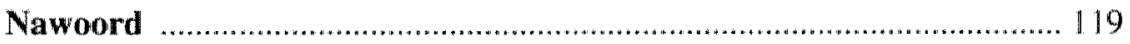

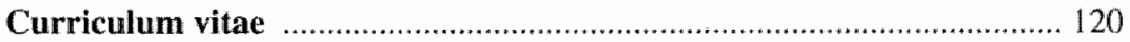




\section{List of abbreviations}

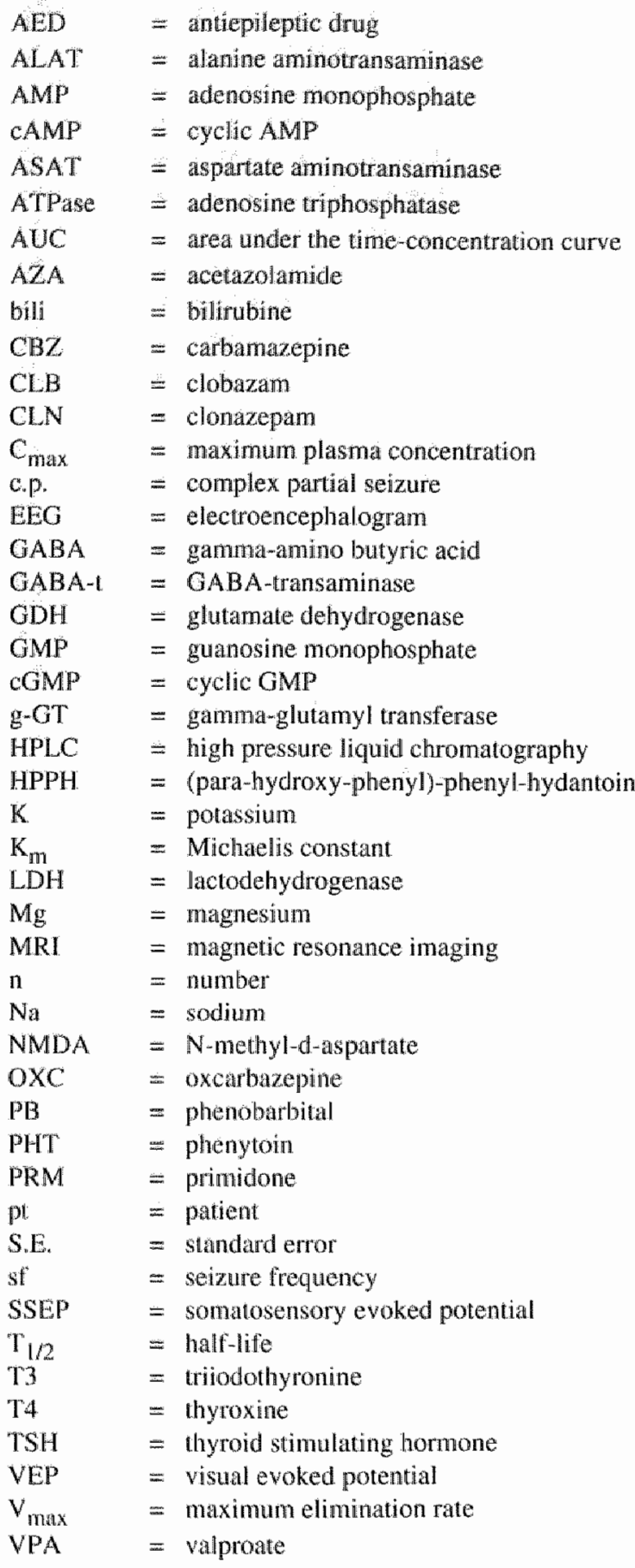

* $R 57720$ and loreclezole are symthesized by Janssen Research Foundation, Beerse, Belgium. 


\title{
Chapter 1. SOME ASPECTS OF EPILEPSY AND THE TREATMENT OF EPILEPTIC SEIZURES
}

\section{1}

\author{
Introduction
}

Between 1 in 250 and 1 in 150 people suffer from epilepsy (Robb 1977. Zielinski 1982, Li et al. 1985, Kerănen et al. 1989, Loiseau et al. 1990). Since the revised classification of epileptic seizures was prepared in 1981 the Commission on Classification and Terminology of the International League Against Epilepsy (ILAE) has been examining the classification of epilepsy syndromes (Commission on Classification and Terminology of the International League Against Epilepsy 1981, 1989). An accurate diagnosis is imperative both for medicinal and for surgical treatment of epilepsy (Janz 1969). In addition to the classification of seizures there is a classification of syndromes. Four major groups of syndromes are distinguished, with various subdivisions.

The existence of two classification systems, one for seizures and the other for epilepsy syndromes, causes much confusion. A given syndrome can manifest itself in various types of seizure, and a given type of seizure can occur in various kinds of epilepsy syndrome. Clinical observation is still invariably the starting point in the diagnosis and treatment of epilepsy (Chadwick 1990a).

The full classifications of syndromes and types of seizure are given in Tables 1.1.a and 1.1.b respectively. Epilepsy syndromes are divided into localization-related, generalized and undetermined epilepsies and special syndromes, whilst seizures are divided into partial, generalized and unclassified epileptic seizures. An epileptic syndrome is an epileptic disorder characterized by a picture of signs and symptoms that usually occur simultaneously. The difference between a syndrome and a disease is that a disease generally has the same cause and the same prognosis, whereas for a syndrome this is not necessarily true. It is important to distinguish between symptomatic (secondary) epilepsy, which has an identifiable cause; idiopathic (primary) forms, whose aetiology is unknow but in which genetic factors may play a role, whilst there is an age indication in addition to clinical and electroencephalographic characteristics; and cryptogenic epilepsies, whose cause is similarly unknown, but in which the epilepsy is assumed to be a symptom of an underlying disease. These cryptogenic epilepsies are age-associated, but have a specific EEG picture. The symptomatic temporal epulepsies include the temporal forms with amygdalo-hippocampal (mesiobasal, limbic or rhinencephalic) seizures. The symptoms include epigastric sensations, strong autonomic symptoms, anxiety and olfactory and gustatory hallucinations (Swartz et al. 1990). The temporal epilepsy category inchudes lateral temporal seizures, which are associated with auditory hallucinations, visual phenomena or speech disturbances.

For frontal epilepsies a distinction is made between seizures originating in the supplementary motor area, the cingulum, the anterior frontopolatr area, orbitofrontal, dorsolateral, opercular, and the motor cortex. Strange motor phenomena, such as complex gestures at the start of the seizure and tonic symptoms, are particular features of seizures arising from the the frontal lobe (Fusco et al. 1990). In the past these seizures were often diagnosed as psychogenic (Wilkus et al. 1990). In frontal forms of epilepsy the seizures 


\section{1. localization-related (focal, local, partial)}

1.1 idiopathic (primary)

- benign childhood epilepsy with centro-temporal spikes

- childhood epilepsy with occipital paroxysms

- primary reading epilepsy

\section{2 symptomatic (secondary)}

- temporal lobe epilepsies

- frontal lobe epilepsies

- parietal lobe epilepsies

- occipital lobe epilepsies

- chronic progressive epilepsia partialis continua of childhood

$>$ syndromes characterised by seizures with specific modes of precipitation

\section{3 cryptogenic}

defined by:

seizure type (see ICES)

clinical features

etiology

anatomical localization

\section{2. generalized}

2.1 - benign neonatal familial convulsions

- benign neonatal convulsions

- benign myoclonic epilepsy in infancy

- childhood absence epilepsy (pyknolepsy)

- juvenile absence epilepsy

- juvenille myoclonic epilepsy (impulsive petit mal)

- epilepsies with grand mal seizures (GTCS) on awaking other generalized idiopathic epilepsies

>epilepsies with seizures precipitated by specific modes of activation

\section{2 cryptogenic or symptomatic}

- West syndrome (infantile spasms, Blitz-Nick-Salaam Krämpfe)

- Lennox-Gastaut syndrome

- epilepsy with myoclonic-astatic seizures

- epilepsy with myoclonic absences

2.3.1 nonspecific etiology

- early myoclonic encephalopathy

- early infantile epileptic encephalopathy with suppression bursts other symptomatic generalized epilepsies

2.3 .2 specific syndromes

epileptic seizures may complicate any disease state

\section{3. undetermined epilepsies}

3.1 with both generalized and focal seizures

- neonatal seizures

- severe myoclonic epilepsy in infancy 
- epilepsy with continuous spike-waves during slow wave sleep

- acquired epileptic aphasia (Landau-Kleffner syndrome)

other undetermined epilepsies

3.2 without unequivocal generalized or focal features

\section{4. special syndromes}

4.1 situation-related seizures (Gelegenheitsanfälle)

- febrile convulsions

- isolated seizures or isolated status epilepticus

- seizures occurring only when there is an acute or toxic event due to fac tors such as alcohol, drugs, eclampsia, nonketotic hyperglycemia.

Table 1. I. a. Intemational classification of epilepsies, epilepsy syndromes and related seizure disorders (ICE), Commission on Classification and Terminology of the Imtemational League Against Epilepsy 1989

Intemational classification of epileptic seizures

I. partial seizures (seizures beginning locally)

A. simple partial seizures (consiousness not impaired)

1. with motor symptoms

2. with somatosensory or special sensory symptoms

3. with autonomic symptoms

4. with psychic symptoms

B. complex partial seizures

1. beginning as simple partial seizures and progressing to impaiment of conciousness

a. with no other features

b. with features as in 1.A.1-4

c. with automatisms

C. complex seizures secondarily generalized

II. generalized seizures (bilaterally symmetrical and without local onset)

A. 1. absence seizures

2. aypical absence seizures

B. myoclonic seizures

C. clonic seizures

II. D. tonic seizures

II. E. tonic-clonic seizures

II. Fi. atonic seizures

III. unclassified epileptic seizures (inadequate or incomplete data).

Table 1.J.b. Classification of seizure types - intemational classification of epuleptic seizures (ICES), Commission on Classification and Terminology of the International League Against Epilepsy, 1981. 
often occur several times per day and in slepp; they are generally of short duration, sel. dom give postictal confusion, and tend to secondary generalization.

The Kojewnikow's syndrome, both the Rasmussen syndrome and the form of rolandic partial epilepsy that occurs in adults and children, also belong to the frontal epilepsy category (Bancaud 1985).

The parictal epilepsies are characterized by sensory phenomena. Uswally these are simple partial (also known as elementary partial) seizures with secondary generallzation. A transition to a complex seizure may occur. The same is true of occipital epilepsies, which are frequently but not necessarily associated with positive (something visible) or negative (deficil) visual sensations.

In localization-related epilepsies the onset of the discharge is (in all probability) focal, whereas in generalized epilepsies both hemispheres are affected by the epileptic discharges from the outset. The epilepsy is undetermined without unequivocal focal or generalized features if there is clinical and electroencephalographic evidence of both partial and generalized seizures and there is no positive evidence of a focal or generalized onset. Mostly it is a matter of epilepsy with nocturnal seizures without aura.

The idiopathic generalized epilepsies are characterized by a generally good reaction to medication and normal development of the patient, unlike the generalized cryptogenic or symptomatic epilepsies and generalized symptomatic (age-related) epilepsies of non specific aetiology.

The classification commission reports that it is convinced that the existing proposal is not fully satisfactory and that classification problems might arise (Watanabe 1989). The commonest seizures are the partial ones, representing $60 \%$ (more than $70 \%$ if rapidly generalizing seizures are inchuded) (Forsgren 1990). A recent studly by Loiseau found that situation-related seizures are the commonest, with an incidence of 29.0 people per 100,000 (Loiseau al al. 1990). Symptomatic generalized epilepsy is relatively uncommon, but this type of epilepsy is most pharmacotherapy resistant. In any case the diagnosis may change in the course of time, with regard to both the type of seizure and the sylidione.

\section{2}

\section{Treatment of epileptic seizures}

The treatment of epilepsy depends mainly on pharmacotherapy; about $70 \%$ of epilepsy patients are kept free of seizures by the use of drugs (Rutgers 1984, Duncan 1991). The range of antiepileptic drugs available for them is limited. Antiepileptic drugs should suppress the paroxysmal clinical symptoms of epilepsy without impairing the normal functioning of the organism (Meinardi 1980).

"The pharmacotherapy of epilepsy has a long history (Dreifuss 1989a). In 1857 Sir Charles Locock reported a clear reduction in seizures after administration of potassium bromide. Although it was not tested in formal studies, this substance found widespread use. Sporadic reports still appear on the use of bromide in the treatment of tonic-clonic 
seizures (Scheunemann 1983, Steinhoff and Kruse 1990, Dehling at al. 1990). The sedadue properties of scopolamine were also put to use (Polet 1911). Later, bromide was largely replaced by sedative substances from the barbiturate group, which were easiet to dose correctly. In about 1935, using electric shock and other techniques in animal models, Merritt and Pumam studied the antiepileptic effect of variants of the phenobarbital structure. This led to the discovery of the antiepileptic effect of phenytoin, which was introduced into clinical practice as an antiepileptic drug (Leppik 1989).

Increasingly, substances with antiepileptic activity are sought systematically on the basis of working hypotheses about substances that have proved effective (Meinardi 1987, Chadwick 1990b). This does not alter the fact that one of the newer, invernationally accepted antiepileptic drugs, valproate, which was synthesized as long ago as 1882 by Burton, was identified as an antiepileptic drug by chance by Meunier in 1963 (Burton 1882, Meunier et al. 1963).

If epileptic seizures are defined as sudden paroxysmal excessive neuronal discharges, it is evident that the discharges are a result of excessive excitation of cerebral systems or of insufficient inhibition of such excitation (Jackson 1890. Fariello 1985, McDonald et a. 1991). From experimental models and human studies it is clear that gamma-aminobutyric acid (GABA), an inhibitory neurotransmitter, plays an important role in epileptic seizures (De Deyn et al. 1990). Another factor is the excitatory action of the glutamate-aspartate complex. How GABA acts in the mammalian cerebrum is only partly known (Meldrum 1990). The GABAergic neurotransmission of the GABA receptor complex is manifested in various effects on the flux of potassium ions, chloride ions and calcium ions (Dichter 1989, Macdonald 1989a). Benzodiazepine receptors form part of the GABA receptor complex (DeLorenzo 1988). There are at least two central types of benzodiazepine receptor, but the specific functions of the various receptors have still not been clarified (Wamsley et al. 1985. Sieghart 1988). Administration of CABA at warious sites in the brain has an inhibitory or excitatory effect, depending on the GABA receptors (Gale 1989). In the caudate nucleus (striatum) and certain regions of the colliculus superior GABA acts as a proconvulsant. Elevation of GABA-levels in the substantia nigra potentiates anticonvulsant activity (Gale 1988). GABA has inhibiting potential on nigral projections from the pars reticularis (Grace and Bunney 11979 . Melis and Gale 1983, Zhang et al. 1991). The net effect of GABA activity on the substantia nigra is disinhibition of the target meurons in thatamus, tectum (colliculus superior) and teginentum. The substances associated with GABA synthesis, and also glutamate dehydrogenase $(\mathrm{ODH})$ and tyrosine hydrox ylase, are important in research into the triggering effect of amino acids in the spike activity of the cerebral cortex (Sherwin et al. 1984, Snead, 1990). Substances that potentiate the inhibitory action of GABA are assumed to be potential antiepileptic drugs. Substances with potential antiepileptic activity are thus GABA agonists (prodrugs), GABA transaminase inhibilors, GABA uptake inhibitors and substances that act at the GABA/benzodiazepine allosteric site (Meldrum 1989).

Three glutamate receptor systems are known: N-methyl-d-aspartate (NMDA), quisqualate and kainate receptors. The NMDA subtype is of greatest interest because 
specific drugs acting on it are avalable (McNamara 1989, Chapman et al. 1991, Liljequst 1991). WMDA receptor blockers inhibit kindling (Callaghan and Schwark 1980 , Bowyer 1982). Several substances with this effect have no antiepileptic activity, however. Similarly, endogenous noradrenaline strongly inhibits epileptogenesis in the kindling model, but has no anticonvulsant activity. The anti-epileptogenic effect is controlled via alpha 2 subtype adrenergic receptors. Clonidine, an agonist of these receptors, does not raise the kindling seizure threshold, however (Gellman et al: 1987). Binding of glutamate to the receptor results in a change in membrane conductance, particularly for sodium and calcium ions. Much is still unknown about the role of glutamate and aspartate in the onset of seizures (Fartello 1985, Marrosu et al. 1990). The relationship between glutamate, aspartate, $\mathrm{GABA}$ and taurine is still unclear.

There are several hypothetical activity models for antiepileptic drugs: substances that act at the level of membranes and synapses, substances that work at the molecular level, modulators, substances that influence receptors, neurotransmitters and peptides, substances that work at cellular level, substances that act at the level of multicellular neuronal synchrony, and substances whose activity has been established on a solely empirical basis (Porter 1985a, 1989a. Dichter 1989). Three types can be distinguished: peptides, monoamines, and aminoacids.

To develop new antiepileptic drugs three strategies can be followed: investigation and imitation of the mechanism of effective antiepileptic drugs, research into membrane receptors and channels and ways in which they may be influenced, and research into the physiology of membrane potentials and the associated spreading of epileptic potentials (Kupferberg 1989). This can be done by systemical investigation of derivatives and analogues of existing antiepileptic drugs, known agonists, and antagonists, and by study in depth of forementioned hypothesis. Antiepileptic activity may be studied by suppression of convulsions, either electrically or pharmacologically generated. Other methods of investigation are in vitro methods: slices of brain cortex, especially mentioned hippocampal slices, and isolated neurons. Kindling models are prominent in these studies (Bolwig 1989, MoNamara 1989). Even the kindling model is no more than a simplification of the complex cerebral mechanisms that come into play in epilepsy (Racine et al. 1989).

For various antiepileptic drugs, working hypotheses were drawn up which had to be rejected after a number of years of study. For instance, it was initially thought that phenytoin acted by influencing the sodium channels, then that it acted on the calcium influx through the cellular and subcellular membrame ends, and subsequently that the subsitance acts by influencing calcium-dependent phosphorylation of synaptosomal proteins (Morselli and Lloyd 1985). A common mechanism in the activity of various antiepileptic drugs in the treatment of tonic, tonic clonic and partial seizures seems to be an influence on the GABA-receptor-ionophore macromolecular complex. Despite continually changing viewpoints, research into the activity of antiepileptic drugs still seems the appropriate way to gain more insight into epilepsy and its treatment (Porter 1990). 
The nature of the disorder for which antiepileptic drugs are prescribed means that they must often be used for many years. For antiepileptic drugs more than for any other drug it is therefore imperative to ensure that they are safe in chronic use (Kuschinsky and Lüllmann 1970, Rall and Schleifer 1990). Some patients receiving pharmacotherapy experience tiresome adverse events. The few epileptics who are treated neurosurgically receive post-operative medication which in some cases can be withdrawn after a number of years (Earl Walker 1977, Polkey 1988).

Most patients obtain sufficient seizure control from just one antiepileptic drug (Reynolds and Shorvon 1981). Monotherapy is simpler for the patient (confusion between different types of drug is impossible); monotherapy is less likely to cause side effects, and both factors improve compliance (Wilder 1987, Porter 1989b. Brodie 1990, Gillham et al. 1990, Sabers 1990). Nevertheless, about $20 \%$ of epilepsy sufferers benefit from the use of more than one antiepileptic drug, which however does not always lead to complete freedom from seizures (Henriksen 1984, Mirales and Leppik 1985, Callaghan and Goggin 1988). To give more epileptics better control of their seizures with fewer adverse reactions, better antiepileptic drugs must be developed that are effective as monotherapy (Koella and Schmutz 1981).

The side effects of antiepileptic drugs may be divided into idiosyncratic and dosedependent (Porter 1985b, Smith and Bleck 1991). In a recent article, however, Keyser et al. described a study of side effects in outpatients in which there was no clear relationship with plasma concentration (Keyser et al. 1990). Side effects were reported in $50 \%$ of the patients on monotherapy and in $65 \%$ of the patients on polytherapy. The idiosyncratic side effects were generally independent of dose and occurred mostly in the first weeks of the treatment. In the course of chronic treatment the dose-dependent side effects became more prominent. Teratogenic effects in pregnant women taking antiepileptic drugs are a separate problem (Bossi et al. 1980, Lindhout and Meinardi 1984, Lander and Eadie 1990). Most side effects of antiepileptic drugs are mild, and there is little variation in side effects from one antiepileptic drug to another. Some antiepileptic drugs have specific side effects (see below). The most important are the side effects on the central nervous system, particularly on higher cerebral functions, such as sedation, drowsiness and impairment of cognition (Eadie 1980b, Trimble 1990, Forsythe et al. 1991). Cerebellar effects such as eye movement disturbances, dizziness and ataxia occur with many antiepileptic drugs in cases of acute intoxication, but can also occur during chronic treatment at prescribed doses. In addition there are side effects on the haematopoietic apparatus such as chronic anaemia due to folic acid deficiency, a negative influence on liver and kidney function, and effects on the skin and skeleton and on endocrine function. By taking an accurate history and performing a physical examination and laboratory tests, the effects of adverse reactions can be traced (Doose 1983). With combinations of antiepileptic drugs the risk of intoxication is much greater owing to drugdrug interactions (Richens 1977, Breimer 1991). What in practice can lead to unrecognized or unexpected adverse events is the use of "household remedies" to treat intercurrent illnesses such as influenza. Though regarded as innocuous, these remedies can interfere with the metabolism and protein binding of the antiepileptic medication. A par- 
ticular problem is interaction with drugs that are prescribed, sometimes chronically, for other diseases. Many people, particularly the elderly, are treated for several diseases with drugs which, in combination with the epilepsy treatment, can lead to intoxication symptoms.

The ideal antiepileptic drug gives complete control of seizures, is effective in all types of epilepsy, has no side effects, is easy to detect in the body, is administered only once or twice per day, is inexpensive, and does not interact with other drugs (Gram 1989). Not surprisingly, the ideal antiepileptic drug has still not been found. Currently the main antiepileptic drugs are carbamazepine, valproate, phenytoin, phenobarbital and related barbiturates, various benzodiazepines and ethosuximide. In addition to numerous experimental antiepileptic drugs there are the less commonly used substances such as acetazolamide, mesuximide and primidone, whilst ACTH is in a category of its own (Famacotherapeutisch Kompas 1990). Vigabatrin has recently become available.

\subsubsection{Phenobarbital}

Phenobarbital, which is still used worldwide, was the first safe and effective antiepileptic drug with relatively few side effects in comparison with bromide (Hauptmann 1912). It is effective against tonic-clonic seizures and to a lesser extent against partial seizures, and is ineffective against absences. It is used to treat status epilepticus, despite the slow onset of its efficacy against this condition (Shaner et al. 1988, Osorio and Reed 1989, Painter 1989, Gabor 1990).

Phenobarbital suppresses abnormal focal discharges in experimental models (Prichard 1980, Prichard and Ransom 1989). It reinforces presynaptic inhibition, but more chiefly it lengthens the duration of postsynaptic inhibitory potentials (Nicoll et al. 1975). At high concentrations phenobarbital suppresses transcellular transport of sodium and potassium; it also reduces calcium influx (Schwartz 1979, Sohn and Ferrendelli 1976). Phenobarbital reportedly influences neurotransmitters, GABA, glutamate, acetylcholine and aspartate (Macdonald and McLean 1982). At high doses there is also suppression of the cytochrome oxidase system, which takes part in the synthesis of high-energy phosphate compounds necessary for the maintenance of membrane potentials (Prichard. 1982). At so-called therapentic plasma concentrations phenobarbital seems to exert its effect by increasing postsynaptic GABAergic inhibition and simultaneously reducing glutamate excitation. The effects seen at high concentrations, especially on ion transport and on high-energy phosphate synthesis, seem to be responsible for the sedative properties of phenobarbital (Morselli 1985). The commonest (about $40 \%$ ) and most harmful side effect is sedation and impairment of cognitive function (Schmidt 1982a). School performance in particular can suffer considerabiy in children treated with phenobarbital (Van der Pol et al. 1991). This may also oecur in cases of intrauterine exposition to phenobarbital (Farwell et al. 1990). Aggressive behaviour and character changes are also common, particularly in children. Although these phenomena are reversible, they may have profound negative effects on personality (Trimble and Cull 1988). If phenobarbital is taken at the end of the day the sedative effect may be less troublesome. Since phenobarbital is no longer the first choice, the recommended plasma concentration in most countries is $10-20 \mathrm{mg} / \mathrm{l}$, which seldom produces serious sedation. Higher doses leading 
to plasma concentrations of $40 \mathrm{mg} / \mathrm{l}$ often give better control of seizures if the low dose is ineffective. Phenobarbital is a potent enzyme inducer (Mattson and Cramer 1989a). Plasma protein binding of phenobarbital, playing a minor role in distribution, is about 50\% (Rust and Dodson 1989). Phenobarbital is about 50\% metabolized to hydroxyphenobarbital and subsequently conjugated to glucuronic acid. Parahydroxylated and about $25 \%$ unmetabolized phenobarbital are excreted in urine (Anderson 1989). The clearance of phenobarbital is much higher when urine is alkaline than when it is acidic (Johannessen 1989). Phenobarbital has an unpredictable effect on the plasma concentration of phenytoin and reduces the plasma concentration of carbamazepine (Section $1.4 .2,1.4 .4)$. In combination with valproate, phenobarbital produces a sharp increase in sedation (Section 1.4.6) (Kutt 1989a). Effective treatment is usually attained at doses of $2-4 \mathrm{mg} / \mathrm{kg} /$ day (Eadie $1980 \mathrm{a}$ ). Thanks to its long plasma half-life of $20-80 \mathrm{~h}$, phenobarbital can be dosed once per day, although more frequent administration is sometimes preferred (Schobben 1979). Phenobarbital should be withdrawn with caution owing to the risk of an increase in seizures or status epilepticus.

\subsubsection{Phenytoin}

Since its introduction in 1938 phenytoin has remained a favourite antiepileptic drug despite its disfiguring side effects such as coarse skin, acne, gingival hyperplasia and hirsutism (Merritt and Putnam 1938). Owing to the favourable ratio between effect, side effects and price it is still commonly prescribed. Phenytoin seldom causes sedation at usual dosages (Reynolds 1989). Sedation occurs at high plasma concentration; dizziness, nystagmus and ataxia are also common at high dosages (Schmidt 1982a). Phenytoin has negative effects on cognition, especially on problem solving and visuomotor tasks (Trimble 1987, Vining 1987). The drug is effective against partial and generalized tonic-clonic seizures (Wilder and Rangel 1989). Phenytoin is also used in the treatment of status epilepticus; it is available for intravenous use (Ramsay 1989). Since phenytoin can induce absences, it is contra-indicated in the treatment of idiopathic generalized epilepsy with seizures of this type (Marescaux et al. 1989).

Although there has been much research into the effects of phenytoin, it is unclear what mechanism is important in its mode of action (Morselli 1985, DeLorenzo 1989). At plasma concentrations at the so-called toxic level, effects on calcium and sodium flux are detectable (Kutt 1977. Woodbury 1982). The effect on calcium and sodium flux is presumably due to phenytoin's property of inhibiting the accunnulation of CAMP and cGMP after depolarization (Ferrendelli 1980). The activity of phenytoin probably depends on a complex of actions: potentiation of GABA-regulated postsynaptic inhibition via a direct effect on chloride ion channels and modification of calcium and sodium transport via the cellular and subcellular membranes.

The usual adult dose of phenytoin is $300-400 \mathrm{mg} / \mathrm{day}(6-10 \mathrm{mg} / \mathrm{kg} / \mathrm{day}$ ) (Eadie 1980a). Since the metabolism of phenytoin can become saturated and the safety margin between therapeutic and toxic concentrations is narrow, dosing is difficult (Rimmer and Richens 1988, Pryka et al. 1991). The so-called therapeutic dose is between 10 and $20 \mathrm{mg} / \mathrm{l}$ (Buchthal et al. 1960). Patients exist, however, who have been effectively treated with much lower plasma concentrations (2-3 mg/l) (Feldman and Pippinger 1976, 
Kur 1982). There are also patients who have benefited from much higher dosages giving plasma concentrations of about $30 \mathrm{mg} / \mathrm{l}$, which however were associated with thresonne side effects in $75 \%$ of the patients (Schmid 1977, Cobos 1987). Phenytoin is $90 \%$ bound to plasma protein (Woodbury 1989). It is largely metabolized to (p-hydroxyphenyl)-phenyl-hydantoin (HPPH) (Browne and Chang 1989). HPPH is eliminated as glucuronide. Since the conversion of phenywoin to HPPH can become saturated, the half-1ife apparently depends also on the plasma concentration (Glazko 1989). An approximate estimation of the "half-life" is tound as $1.5 \mathrm{x}$ plasma concentration (mg/l) $+10 \mathrm{~h}$ (Schobben 1979). Thus the half-life is usually between 24 and $40 \mathrm{~h}$. Phenytoin causes enzyme induction and interacts unpredictably with phenobarbital (both raising and lowering the plasma concentration), with carbamazepine (usually lowering of both concentrations) and with valproate, hastening the onset of adverse events (Kutt 19896 , Ichikou et al. 1990). When phenytoin is combined with valproate, phenytoin plasma protein birding decreases and the concentration in the brain increases (Meinardi et al. 1983). Recently, Zifkin e.a warned against combination of phenytoin and clobazam, whereas this combination can cause severe intoxication (Zifkin et al. 1991). Although some patients readily tolerate a single daily dose (owing to the long plasma half-life), two doses per day are usually preferred so that an absorption peak can be avoided.

\subsubsection{Ethosuximide}

Ethosuximide is the most effective member of the succinimide group, which was synthesized around 1950.

The mechanism of action of ethosuximide is unknown (Morselli 1985 , Ferrendelli and Holland 1989). At low doses it inhibits the activity of $\mathrm{Na}^{+}$and $\mathrm{K}+$ ATPase, but not $\mathrm{Mg}^{2+}$ ATPase (Gilbert and Wyllie 1974). In chronic use ethosuximide reduces the level of $\gamma$-hydroxybutyric acid in the brain, which is interesting because $\gamma$-hydroxybutyric acid can induce absence-like attacks in animal models (Erwin and Dietrich 1973 , Godschalk et al. 1977, Snead $1978 a, b, c)$. The possibility that ethosuximide might exert its effect by increasing the GABA-controlled inhibition of cortical neurons is fairly improbable since ethosuximide causes a reduced GABA response in cortical cell cultures (Bames and Dichter 1984). Action via a reduction in the quantity of $\gamma$-hydroxybutyrate as mentioned above is more likely. Ethosuximide is the first safe and effective drug against absences in idiopathic generalized epilepsy (Sherwin 1989). It is not indicated in tonic-clonic seizures and is not very effective against partial seizures (Subirana and Ollet-Daurella 1964). Ethosuximide is generally well tolerated; gastrointestinal side effects, irritability and sleep disturbances may occur (Dreifuss 1989b). Psychosis is a possible serious side effect, occurring mainly in adults in whom there is a drastic reduction in seizures. It has also been reported accompanying extremely high plasmalevels (Van Wieringen and Vrijlandt 1983). The substance is rapidly absorbed and the plasma halt-life is between 49 and $66 \mathrm{~h}$ in adults and about $30 \mathrm{~h}$ in children (Buchanan et al. 1973). Plasma protein binding is negligible (Perucca and Richens 1980). Ethosuximide is converted into various metabolites; less than $20 \%$ is excreted unchanged in the urine (Chang 1989a,b). Plasma concentrations in effective treatment are between 40 and $90 \mathrm{mg} / \mathrm{l}$. In children the usual dose is $750-1000 \mathrm{mg} / \mathrm{day}(25-35 \mathrm{mg} / \mathrm{kg} /$ day) (Eadie 1980 a). Two doses per day are usual at higher dosages. 
Carbamazepine is a popular antiepileptic drug which was synthesized in 1957 and has been available in most west European countries since the sixties (Kraemer and Hopt 1987). Carbamazepine is only sparingly soluble in water and in fat, but is readily solu ble in benzene and absolute allcohol (Sillanpiata 1981). Once absorbed, the substance is distributed rapidly and evenly thronghout the body (Schobben 1979). Absorption depends on the dosage form of carbamazepine; the various formulations can produce different plasma concentration profiles over time, which can lead to intermittent side effects (Morselli 1989, American Academy of Neurology 1990, Nuwer al al. 1990, Rentmeester ett al. 1990, Rentmeester 1991). Although the efficacy profile of carbamazepine is similar to that of phenytoin, the assumed mechanism of action is different (Porter 1982). In particular, the effect on transmembrane ion transport is detectable only at very high doses (Schauf et al. 1974). Carbamazepine has an effect on cyclic nucleotides and adenosine receptors (Morselli 1985). It seems to be a partial agonist of adenosine receptors (Skerritt et al. 1983a,b, Marangos et al. 1985). This, together with interaction with the noradrenergic system, is probably relevant to the antiepileptic activity of carbamazepine. Action of carbamazepine is likely to be both pre-and postsynaptically by blocking release of neurotransmitter by firing action potentials and postsynaptically by blocking the development of high frequency repetitive discharge initiated at cell bodies (Macdonald 19896).

Carbamazepine has good efficacy, particularly in partial epilepsy, and the side effects are usually mild (Sullivan et al. 1981, Gram and Klosterskov Jensen 1989, Spiller et al. 1990). The incidence of adverse events is $33 \%$ in monotherapy and $47 \%$ in polytherapy (Schmidt 1982a). Most side effects are transient and are reversible after withdrawal of carbamazepine. Some are dose-dependent, such as somnolence, nausea, double vision, dizziness and ataxia (Höppener et al. 1980). Only 5\% of patients have serious side effects requiring withdrawal of the medication. In about $4 \%$ there is exanthem; bone marrow depression, aplastic anaemia, leucopenia or lupus-erythomatosus like syndrome are rare (De Giorgio et al 1991). In the event of rash, this side effect can be suppressed by giving prednisone, if necessary in combination with an antihistanine, and the therapy can be continued (Murphy et al. 1991). Administration of carbamazepine must lake account of changes in the patient's metabolism. In healthy wolunteers the half-life is 19 55 h (Faigle and Feldmann 1989). In chronic treatment the half-life is $14-27 \mathrm{~h}$ and can fall still further at the high doses that are sometimes necessary for effective treatment (Theisohn et al. 1983). When phenytoin or phenobarbital is used as comedication, halflives of less than $7 \mathrm{~h}$ can occur. Binding to plasma proteins is $70-80 \%$; at high dosages in combination with a strong protein binder such as valproate, intoxication symptoms are observed at so-called therapeutic values (Pitlick and Levy 1989). An important met. abolite with antiepileptic activity is carbamazepine-10,11-epoxide, which is assumed to be responsible for some of the side effects (Lindhout et al. 1984, Kerr and Levy 1989, Pisani et al. 1990, Tomson et al. 1990, Dhuna et al. 1991). Actually, carbamazepine is currently believed to be the least harmful antiepileptic drug if an antiepileptic drug is indicated in pregnancy, although spina bifida is more frequent than with the most commonly used antiepileptic drugs, except valproate (Dam and Philbert 1984, Dravet et al. 1989, Rosa 1991). Besides being effective in partial epilepsy, carbamazepine is also 
prescribed in generalized epilepsy, including the idopathic form with tonic-clonic seizures (Loisean and Duche 1989). Carbamazepine is contraindicated in the treatment of absence seizures in diopathic generalized epilepsy, as it can provoke absence seizures and absence status (Shields and Saslow 1983, Horn et al. 1986, Marescaux et al. 1989). In adults the carbamazepine dosage is generally $600-1200 \mathrm{mg} /$ day $(15-20 \mathrm{mg} / \mathrm{kg} / \mathrm{day})$. The number of doses per day depending on the dosage form (Hartley et al. 1991). In the most favourable case only one dose per day is required; conventional dosage forms are usually taken three or four times per day (Hulsman et al 1989, Dhalla et al. 1991).

\subsubsection{Valproate}

Valproate has been the drug of first choice in all forms of idiopathic generalized epilepsy since its introduction as an antiepileptic drug in about 1970 (Bourgeois et al. 1987 , Borgeois 1989).

For many years the antiepileptic activity of valproate was attributed to the increase in GABA in the brain resulting from delayed metabolism of GABA (Godin et al. 1969, Sawaya et al, 1975). Valproate is a weak GABA transaminase inhibitor, but is a much stronger inhibitor of succinyl semialdehyde dehydrogenase (Van der Laan et al. 1979). Valproate enhances nigral GABA levels and limits sustained repetitive firing at therapeutical levels (Fariello and Smith 1989). Otherwise the increase in GABA is modest, and the antiepileptic effect of valproate is still detectable at GABA concentrations that are much lower than the usual anticonvulsant concentrations (Lacolle et al. 1978). The effect of valproate on the GABA system is difficult to isolate from the other effects that occur during chronic treatment with valproate (Gram 1988, Gram et al. 1988). Increases in aspartate, glutamate, taurine and glutamine in the brain are particularly striking (Williams et al. 1980). It is possible that metabolites may determine the efficacy of valproate in the treatment of epilepsy (Morselli 1985, Semmes and Shen 1991). About 25\% of users experience side effects, some of which are innocuous as regards toxicity but do impair the quality of life, e.g. gastric discomfort, nausea and alopecia (Schmidt 1982a). Increase in body weight can be severe. Usually reduction of caloric intake is sufficient to counteract this problem (Dreifuss 1989c). On the other hand there are also patients who lose weight during valproate treatment, although they are fewer. In combination with phenobarbital, valproate can lead to serious sedation due to an increased plasma concentration; the sedation subsides when the phenobarbital dose is reduced (Gram et al. 1977). As valproate is about $95 \%$ bound to plasma protein, it interacts competitively with other drugs that allso bind strongly to plasma protein (Haidukewych and Rodin 1981. Hulsman et al. 1984, leti et al. 1990). Combination with phenytoin produces adverse events in virtually every case, just like combination with carbamazepine (Rentmeester and Hulsman 1988). Valproate is metabolized in a 20 metabolites (Baille and Rettenmeier 1989). "The plasma half-life is about $16 \mathrm{~h}$, but seems to be of little value in calculating the dosage interval. Valproate is given 1-4 times per day, sometimes even more frequently (Cenraud et al. 1981, Covanis et al. 1981, Rowan et al. 1981). Valproate is absorbed rapidly after oral administration (Levy and Shen 1989a). The maximum plasma concentration is reached after $0.5-5 \mathrm{~h}$, depending on the properties of the dosage form (Schobben 1979, Berger and Loiseau 1986). The strong fluctuations in plasma valproate concentration during the day are presumably the cause of the intermit- 
tent infoxication that occurs during combination therapy with valproate (Rodin and Haidukewych 1981, Mattson and Cramer 19896). Side eflects can be reduced by changing to a long-acting formulation which produces much less fuctuation in blood levels (Rentmeester and Hulsman 1989). Other side elfects are thrombocytopenia, lymphopenia and - at higher dosages - tremor. In babies and toddlers caution is necessary in view of the risk of lethal liver function disturbances (Scheffner et al. 1988, Dreifuss 1990, Hulsman et al. 1984, Hulsman 1989). Such disturbances are possible in older patients too, but are then reversible if diagnosed promptly. Polyphamacy in young patients leads to a much greater risk of lethal complications. In young children, aggressive and agitated behaviour has been reported with an incidence of up to $64 \%$ (Herranz et al. 1982). Use during pregnancy is contra-indicated in view of the greatly elevated risk of serious foetal malformations, especially neural tube defects (often with hydrocephalus, and frequently with other midline or fusion defects) (Jeavons 1984, Lindhout and Meinardi 1984, Lindhout and Comel 1989). This serious malformation can often be detected prenatally, however (Van Gool and Theunissen 1990). Only when the threat to mother and foetus from relapsing tonic-clonic seizures leaves no alternative is there an indication for valproate in pregnancy. In Lennox-Gastaut syndrome valproate is effective in combination with benzodiazepines (clonazepam, nitrazepam) (Matthes 1977). In very high dosages (200-300 $\mathrm{mg} / \mathrm{kg}$ body weight/day) given for a short period, valproate has produced a favourable effect in West syndrome (Kotlarek et al. 1989, Garaizar and Prats 1989). However, this form of epilepsy occurs at just the very age at which the risk of hepatotoxicity is greatest. Valproate is also prescribed for partial epilepsy, in which its efficacy seems to be comparable to that of carbamazepine (Chadwick 1987, Dean and Penry 1988, Scollo-Lavizzarri 1989). The usual adult dose is $15-30 \mathrm{mg} / \mathrm{kg} / \mathrm{day}$. In elderly epilepsy patients the substance is well tolerated and is effective in all forms of epilepsy (Andersen and Pedersen 1989, Pedersen and Anderson 1990).

\subsection{6}

Benzodiazepines

Since their introduction in the sixties benzodiazepines have formed a separate group of antiepileptic drugs inasmuch as they all show some degree of anticonvulsant efficacy (Lanoir et al. 1964). In nearly every case the effectiveness of benzodiazepines is transient, owing to the onset of tolerance (a reduction or disappearance of the effect), although clobazam seems somewhat favourable in this respect (Sternbach 1983, Rosenberg el al. 1989, Shorwon 1989, Guberman et al. 1990, Canadian Clobazam Cooperative Group 1991).

Benzodiazepines act at specific benzodiazepine recognition sites (receptors) of the GABA macromolecule complex (Haefely 1980, 1989, Costa et al. 1983). These receptors are highly specific with regard to the anticonvulsant activity of benzodiazepines, since other anxiolytics do not exert an anticonvulsant effect at these sites (Meldrum and Braestrup 1984). Benzodiazepines modulate the GABAergic synaptic transmission in such a way that this leads to frequent opening of the chloride channels (Molnler and Richards 1983). This effect has been confirmed by the discovery of diazepam binding inhibitors, which have a regional distribution over the central nervous system and thus similarly have a modulatory effect (Ferrarrese et al. 1989). These effects are detectable both in vitro and in vivo. Whether all the effects of benzodiazepines are exerted via an 
influence on GABAergic transmission is still unknown (Morselli 1985). There are indications that a reduction in benzodiazepine receptors exists at the foci in localizationrelated epilepsy (Savic et al. 1988). In diopathic generalized epilepsy no focal reduction is detectable (Savic et al. 1990).

Benzodiazepines are particularly indicated in acute situations, especially in status epilepticus (Treiman 1989). Clonazepam, diazepam, midazolam, clorazepate and lorazepam have shown their walue here (Mitchell and Crawford 1990). Diazepam in the form of rectioles (rapidly absorbable solution in containers for rectal administration) has amply proved its value for the treatment of serial attacks or status epilepticus in unhospitalized patients and in cases of febrille convulsions (Lombroso 1989, Schmidt 1989). All benzodiazepines produce sedation as a side effect, although clobazam is favourable in this respect (Koeppen et al. 1987). Paradoxical reactions, especially psychotic states and depression, are feared side effects of benzodiazepines. Children may become agitated and irritable (Sato 1989). Hypersalivation and viscous mucus are sometimes reasons for withdrawal in babies and small children. Nevertheless, benzodiazepines are extremely safe drugs if they are prescribed as monotherapy (Prescott 1983). Benzodiazepines can be effective in all forms of epilepsy, but have proved their value above all in status epilepticus, Lennox-Gastaut syndrome and partial epilepsy (Browne and Feldmann 1980, Obeid and Panayiotopoulos 1989, Baruzzi et al. 1989, Wilenski and Friel 1989). Benzodiazepines are extensively metabolized, and many metabolites are pharmacologically active (Kaplan 1980, Kaplan and Jack 1983). Despite high plasma protein binding (about $95 \%$ ), accumulation in the brain is rapid; the substances are strongly lipophilic (Schobben 1979). The plasma concentrations necessary for effective treatment vary widely, and their practical use for the treatment of the patient is still unclear. Benzodiazepines are extremely useful for short or intermittent use (Van Heycop ten Ham 1974)

\subsubsection{Vigabatrin}

$\gamma$-Vinyl- $\gamma$-aminobutyric acid, or vigabatrin, is the first registered antiepileptic drug to have been developed on the basis of current theories and hypotheses about epilepsy (Gram et al. 1989a). $\gamma$-Aminobutyric acid (GABA) has an inhibitory effect on neurotransmission in the brain (Section 1.3). By inhibiting the degradation of GABA in the brain it should therefore theoretically be possible to suppress excessive excitatory transmission such as occurs in epilepsy (Meldrum 1989). Vigabatrin is an irreversible inhibitor of GABA transaminase (GABA-t). Vigabatrin inhibits neuronal GABA-t preferentially. Apart from a much weaker effect on glutamate decarboxylase, this effect is specific, thus emphasizing the GABAergic action of vigabatrin (Ben-Menachem 1989). Administration of vigabatrin raises the GABA concentration in the brain and cerebrospinal fluid (Halonen et al. 1991).

After chronic administration to rodents for 3 months, microvacuoles are detectable in the nerve sheaths, particularly in the optical pathways of rat brains (Graham 1989). These effects were allso seen in dogs at doses of $30-50 \mathrm{mg} / \mathrm{kg}$ body weight/day. In dogs protraction of somatosensory potentials (SSEP) was seen (Arezzo et al. 1989). These phenomena seem to be fully reversible after withdrawal of vigabatrin. In monkeys the brain changes are minimal or questionable. In humans neurological side effects of this 
Tye are not observed: evoked potentials (SSEP, VEP), nagnetic resonance inaging (MRI), neuropsychological findings and the EEG are completely normal (Cosi et al. 1989. Liegeois-Chauvel et al. 1989, Kalviänen et al. 1990, Thomas et al. 1990). About 50 patients who were treated with vigabatrin have since died, due to events not related to the use of vigabatrin. On extensive neuropathological investigation none of them showed any evidence of oedema or vacuolization (Cannon at al. 1990). Patients who have undergone brain surgery, similarly showed neither microvacuolization nor any other abnormalities attributable to vigabatrin (Trottier et al. 1987, 1989).

Vigabatrin is absorbed rapidly; it reaches a plasma concentration peak $1-2$ h after oral administration. The absorption of vigabatrin is not influenced by meals (FriskHolmberg et al. 1989). Vigabatrin does not bind to plasma proteins and is excreted largely unchanged by the renal route (Schechter 1989). The plasma half-life of vigabatrin is 5-8 h. No degradation products of vigabatrin have been detected.

The elimination of vigabatrin follows a fitst order process. The effects of vigabatrin on the GABA-t in the platelets are detectable from $1 / 2 \mathrm{~h}$ to $72 \mathrm{~h}$ after administration. The pharmacological effects in the platelets after $24 \mathrm{~h}$ are still the same as the effects after $12 \mathrm{~h}$ (Bolton et al. 1989). The effect on GABA-t in the platelets is fully reversible after withdrawal of vigabatrin. Because the effect of vigabatrin depends on the rate of synthesis of GABA-t, there is no correlation between the plasma concentration of vigabatrin and its affect. Determination of the plasma concentration of vigabatrin seems to be of little use in the treatment of epilepsy. In chronic administration there is no diminution of the effect of vigabatrin on GABA-t. In the vigabatrin studies in which more than 2000 patients were treated for periods of up to 6 years or more, more than $40 \%$ of the patients with refractory epilepsy showed a reduction of more than $50 \%$ in the rrequency of seizures (Browne et al. 1989, Ben-Menachem 1989). It was found that $20 \%$ of the patients were completely free of seizures with vigabatrin. Vigabatrin seems to be especially indicated in localization-related epilepsy (Michelucci and Tassinari 1989). It is particularly effective in the treatment of partial seizures, with or without secondary generalization (Ring et al, 1990). Vigabatrin treatment has been studied in children suffering from localization-related epilepsy; as in adults, the efficacy is sometimes sulficient to give complete freedom from seizures if vigabatrin is given as add-on therapy (Livingstone et al. 1989, Luna et al. 1989). Children with infantile spasms have also been treated (Beaumont et al. 1990); in 50\% of them the seizures were reduced by more than $50 \%$ and the effect persisted.

The usual adult dose is $2-4 \mathrm{~g}$ vigabatrin/day. In children the recommended dose is $1 \mathrm{~g} / \mathrm{day}$ between the ages of 3 and 9 and $2 \mathrm{~g} / \mathrm{day}$ for older children. Vigabatrin is given twice per day; more frequent administration cloes not seem to confer any advantage. When vigabatrin is withdrawn the dose must be reduced gradually over $2-4$ weeks to guard against the risk of withdrawal seizures, which exists even if the treatment has had no effect on the frequency of seizures (Ried and Schmidt 1989).

Vigabatrin is not free from adverse reactions: the side effects are similar to those of the older antiepileptic drugs, e.g. dizziness, headache, somnolence and weight gatn. Agitation, confusion, nervousness and psychosis appear to be serious side effects 
(Robinson and Richens 1990). In a group of more than 200 patients treated by Sander et a) 15 patients $(=7.5 \%$ ) becane pychotic (Sander et al. 1990,1991$)$. It is striking that almost all these people were taking normal doses of vigabatrin. Ten of them had no history of psychological dysfunction. Although there is still no evidence that vigabatrin is harmful to the human foetus, its administration during pregnancy is not recommanded. If therapy with vigabatrin is started shorty after delivery breast feeding is not recommanded either.

Interaction with existing antiepileptic drugs: the plasma concentration of phenytoin showed a reduction of $10-20 \%$ in a number of patients; less frequently there was a slight decrease in the concentrations of phenobarbital and primidone $(7-1.1 \%)$, whilst no effect was found on levels of carbamazepine, valproaie and benzodiazepines (Rimmer and Richens 1989). There is no evidence of enzyme induction by vigabatrin.

\subsection{Imidazoles and triazoles}

Many substances have now been discovered that have antiepileptic properties in animal studies. Various drugs have also been developed that theoretically should have anticonvulsant activity. Examples of these are calcium entry blockers such as flunarizine, the glycine prodrug milacemide, and the glutamate antagonist lamotrigine (Overweg et al. 1984. Houtkoper et al. 1986, Gram 1989b). The new class of antiepileptic drugs also includes the imidazoles and trizzoles. The imidazoles include nafimidone, which is rapidly converted in animals and in man and has shown antiepileptic activity in animal studies (Graham et al. 1983). Nafimidone exhibits antiepileptic activity in animalmodels and in human investigations (Treiman et al. 1985). Kapetanovic and Kupferberg investigated nafimidone for inhibition of microsomal metabolism of phenytoin and carbamazepine (Kapetanovic and Kupferberg 1984). Rat liver microsomes pretreated with phenytoin were extracted and incubated with nafimidone and reduced nafimidone. Both were found to inhibit the p-hydroxylation of phenytoin, the inhibition depending on concentration. Similar results were found in studies of carbamazepine.

Nafimidone was investigaled as an antiepileptic drug in 6 epilepsy patients (Treiman et al. 1984). In add-on treatment the plasma concentrations of carbamazepine and phenytoin rose. Durng treatment with nafimidone the carbamazepine clearance liell 4.2 to 10 times and phenytoin clearance fell 1.8 to 3.6 times compared to baseline clearance before adding nafimidone. It was concluded that nafimidone appears to be a potent inhibitor of carbamazepine and phenytoin metabolism. Owing to interaction with the existing antiepileptic drugs, antiepileptic efficacy could not be evaluated in this study. In a study by Kapetanovic and Kupferberg the influence of nafimidone on the metabolism of phenytoin and carbamazepine was demonstrated (Kapetanovic and Kupferberg 1985). An unknown metabolite of phenytoin was found. Also an accelerated metabolism of carbamazepine was observed, leading to elevated plasma concentrations of carbamazepine-10,11-epoxide. The antiepileptic efficacy of this metabolite of carbamazepine has been repeatedly reported (Graves et al. 1988, Tomson et al. 1990). By raising the blood level of this substance the efficacy can be wrongly attributed to the test drug itself, whereas the therapeutic effect might just as easily be attributed to the existing antiepileptic drugs. This problem can be only partly obviated by maintaining constant blood 
levels of the existing antiepileptic drug (parent substance) because the degradation products may themselves have a considerable antiepileptic effect. Moreover, maintenance of constant plasma concentrations of the comedication poses great problems in designing double-blind studies. The experimental antiepileptic drugs R 57720 and loreclezole described in this study are an imidazole and a triazole respectively.

The great affinity of 4(5)-imidazoles for cytochrome P-450 is apparently responsible for the inhibition of epoxidation and of hydroxylation (Wilkinson et al. 1972, 1974, Palmer and Cawthorne 1973, Hajek et al. 1982). The great lipophilicity of the 4(5)-substituted substances facilitates penetration of lipid membranes and the hydrophobia facilitates binding to cytochrome P-450. Enzyme inhibition depends primarily on lipophilicity (Workman et al. 1983). Imidazoles stimulate cyclic nucleotide phosphodiesterase. This has led to the hypothesis that imidazoles can potentiate the action of substances that supposedly act via cAMP. In studies of the mechanism of action of imidazoles the effect on the metabolism of other substances must be evaluated critically (Kapetanovic 1990).

\subsubsection{R $\quad R 7720$}

R $57720\left(=(\mathrm{Z})\right.$-1- $\left[2\right.$-chloro-2-(2,4-dichlorophenyl) ethenyl]-1 $\mathrm{H}$-imidazole $\left.=\mathrm{C}_{11} \mathrm{H}_{7} \mathrm{Cl}_{3} \mathrm{~N}_{2}\right)$ (Fig. 1.5.a) is an imidazole derivative with anticonvulsant and antihypoxic properties which have been demonstrated in various animal experiments (Wauquier et al. 1983). The substance has an antiepileptic spectrum similar to that of diazepam or phenobarbital, and is more effective than phenytoin, carbamazepine, valproate and ethosuximide. In acute administration to animals the lethal dose $\left(\mathrm{LD}_{50}\right)$ was found to be about 13 times the effective dose $\left(\mathrm{ED}_{50}\right)$. In chronic administration slight changes in haemoglobin, haematocrit and erythrocyte count were observed only at high doses in rats; in dogs the weight gain was lower than in the controls. Rat studies showed no enzyme induction. Mutagenicity was undetectable even at cytotoxic doses (Ames Salmonella microsomal activity test). Chapter 2 gives further details of clinical studies of R 57720 . All that is known about the mechanism of action of R 57720 is that, like substances that are known to exert GABA-agonistic activity, it increases the binding of diazepam and flunitrazepam.

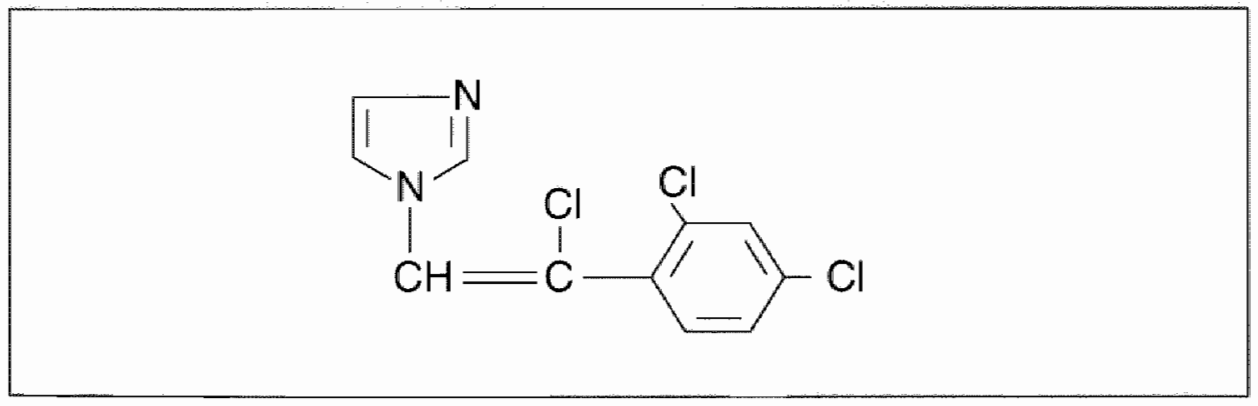

Fig. 1.5.a. Two-dimensional representation of the molecular structure of $R 57720$. 
Loreclezole $(=(Z)-1-[2$-chloro-2-(2,4-dichlorophenyl $)$ ethenyl]-1] $\mathrm{H}-1,2,4$-triazole $=$ $\mathrm{C}_{10} \mathrm{H}_{6} \mathrm{Cl}_{3} \mathrm{~N}_{3}=\mathrm{R} 72063$ ) (Fig. 1.5.b) is a triazole derivative with powerful anticonvulsant properties which have been demonstrated in various animal models and various seizure models (Wauquier et al. 1988a, Jenkins et al. 1988).

Loreclezole is effective in so-called genetic models, in experimental animals with reflex epilepsy, and in all models in which seizures are triggered by electric or chemical stimulation (Wauquier et al. 1990). Loreclezole protects the experimental animals against tonic and clonic seizures; in these studies the antiepileptic profile is comparable to that of diazepam or phenobarbital. Provocation tests with allyl glycine reveal a profile comparable to that of valproate, trimethadione or ethosuximide. Thus loreclezole is both effective in studies designed to investigate the spreading of epileptic activity, as those in which the excitation threshold is investigated. From these profiles it may be hypothetically inferred that loreclezole might have some action on the $\mathrm{GABA}_{\mathrm{A}}$ receptor complex (Wauquier et al. 1990). The overall effect of loreclezole suggests efficacy through an action on the benzodiazepine receptor. Although the competitive benzodiazepine antagonist flumazenil does not reverse the effect of loreclezole, the pyrazoloquinoline derivative CGS 8216 , a substance that acts noncompetitively at the benzodiazepine receptors, does antagonize the effect of loreclezole (Czernik et al. 1982). Loreclezole seems to influence the $\mathrm{GABA}_{\mathrm{A}}$-receptors in a unique way. A plasma concentration of about $1 \mathrm{mg} / \mathrm{l}$ was effective in dogs (Wauquier et al. 1988b,c). Single doses and long-term administration of loreclezole were well tolerated in volunteers. Single dose administration of $100 \mathrm{mg}$ loreclezole in volunteers resulted in slight sedation in some subjects (Arends et al. 1988). Single dose administration of $100-150 \mathrm{mg}$ in patients with photosensitive epilepsy resulted in decreased paroxysmal activity on EEG in all patients tested (Overweg and De Beukelaar 1990). Loreclezole is absorbed rapidly and efficiently; maximum blood levels are reached $1-2 \mathrm{~h}$ after oral administration (Van de Velde et al. 1987, 1988a,b). The terminal half-life is of the order of 25 days. This thesis is mainly devoted to loreclezole; Chapters 3 to 8 deal with the clinical aspects of the substance.

As stated in Section 1.4, there is a limited arsenal of effective antiepileptic drugs with which a proportion of epilepsy sufferers can be effectively treated without the stress of

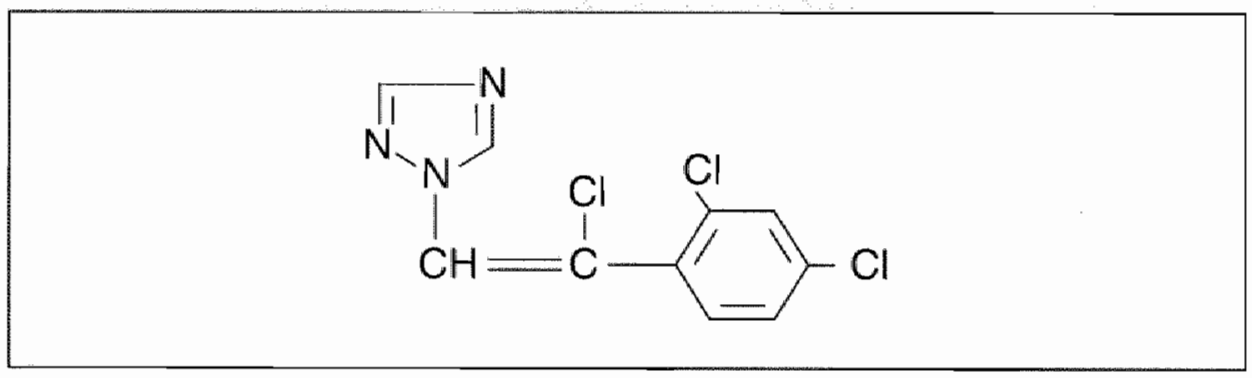

Fig. 1.5.b. Two-dimensional representation of the molecular structure of loreclezale. 
harmful side effects. Those patients who are beyond the scope of the beneficial effects of available antiepileptic drugs, are suitable for investigations with experimental drugs. The Commission on Antiepileptic Drugs of the ILAE has drawn up guidelines for the various phases of research into new antiepileptic drugs (Commission on Antiepileptic Drugs 1989). Special problems rise in the treatment with experimental antiepileptic drugs in children, due to age-related aspects (Commission on Antiepileptic Drugs 1991).

\subsubsection{Various stages in patient studies}

When an experimental substance has demonstrated its safety in animal experiments, it is administered to volunteers in single and multiple doses and at various dosages. Once thorough investigation in volunteers has shown that the substance is also safe in man, initial studies are performed in which the substance is administered to patients with epilepsy. Antiepileptic drugs must be used for long periods. The patient will not obtain freedom from seizures from a short course of treatment, and so the overriding requirement is that the substance must be safe. Short-term administration is useful, however, in giving some indication of acute toxicity and of interactions with existing antiepileptic medication. Electroencephalography after a single dose of the substance in photostimulation-sensitive patients gives an indication of whether or not a hurnan antiepileptic effect exists (Binnie 1982, Kasteleijn-Nolst Trenité 1989).

As already stated, the pharmacotherapy of epilepsy requires chronic administration of antiepileptic drugs. Withdrawal of the medication carries the risk of an increase in the frequency of seizures or of onset of status epilepticus (Reynolds and Shorvon 1980). Accordingly, experimental antiepileptic drugs are usually introduced by adding them to the existing medication. This is known as add-on therapy. Only when seizure control has shown a definite improvement after addition of the experimental antiepileptic drug can reduction of the polypharmacy be considered. Initial studies of a potential antiepe ileptic drug in patients are usually performed in the open mode in small groups of patients (about 10) so that adverse reactions and interactions with existing antiepileptic drugs can be identified as promptly as possible (Meijer 1991). In addition an open study gives some indication of dosage and efficacy. To confirm the efficacy, however, randomized, placebo-controlled double-blind studies are necessary (Gram et al. 1981, White and Cereghino 1981). The size of the sample will be determined on the basis of various items, like trial design, expected efficacy and profile of the new drug. The study design may be parallel or cross-over (Scoville et al. 1981). One of the advantages of a crossover design is that it makes more effective use of the available patients. To prevent a carry-over effect a cross-over study must include a wash-out period, which depends on the half-life of the test substance (Gram et al. 1982, Johnson 1984). A cross-over study is of course unpractical if the plasma half-life of the test substance (like loreclezole) is longer than a few days. In follow-up studies persistence of antiepileptic effect over a longer time can be evaluated, as well as efficacy of the new drug in monotherapy by withdrawal of previous therapy. For better assessment of efficacy and long term safety studies in larger groups should be performed. Various, randomized designs can be employed. In general a number of $300-400$ patients should be included; at least 100 - 150 patients should be treated for more than a year. Intensive monitoring of possible effects on all systems should be performed. After these studies the drug may be marketed. 
In the first instance, new or potential antiepileptic drugs are used in patients in whom the existing antiepileptic drugs give insufficient suppression of seizures or induce intolerable side effects. Therefore, a new drug always has a difficult start if it is investigated in patients who evidently have a type of epilepsy that is not helped by conventional drugs. Such an investigation carries the risk that a good antiepileptic drug might not be recognized as such because the epilepsy of the treated group of patients is resistant to all forms of phamacotherapy (Johnson 1984, Binme 1990). Ethically it is more responsible to treat people in whom every known and demonstrably safe therapy has failed. The aim of the extensive animal and volunteer studies is to show with the greatest possible probability that an experimental drug will not be harmful when administered to patients (Kupferberg et al. 1981). Assessment of risk is far more difficult than is the determination of the efficacy of a new drug. In the last phase before marketing about 500 - 2000 selected patients will receive the new drug. The number of patients on long term therapy is even more limited, so medically impontant risks, that are delayed or less frequent than once per 1000 administrations, may be revealed only after the drug is used broadly (Nies 1990). The same can be stated for use during pregnancy. In fact at present there is a dilemma with respect to pregnancy. Its use is not permitted in pregnancy unless an indication of possible risks has been obtained. On the other hand no ethical committee will agree with a study design testing the safety of a drug during pregnacy. Only in monotherapy can a clear side effects profile of the substance be obtained. For these reasons post-marketing surveillance is mandatory (Stricker 1989).

\subsubsection{Inclusion and exchusion criteria}

To minimize the risks associated with the study and to guarantee that the results will be capable of being evaluated scientifically, a number of inclusion and exclusion criteria are imposed. These criteria may vary from one study to another. Drop-outs must be prevented as far as possible to avoid problems with the evaluation of the study group (Schoonbrood-Lenssen and Starrenburg 1990). For the testing of antiepilleptic drugs a number of inclusion and exclusion criteria are more or less self-evident. For most investigations there are minimum and maximum ages. The epilepsy must be properly diagnosed and well defined. To enable the effect of the new treatment to be measured, a new situation must arise - or be capable of arising - within a reasonable period after addition of the new drug. In practice this means that before the start of the study a certain number of seizures must have occured within a given period of time (commonly used numbers are 4 - 10 seizures in one monih) so that it is possible to check whether there is any measurable change in the number of seizures after administration of the new drug. The ideal goal is of course that there will be no more seizures. Resistance of the disease to classical pharmacotherapy is an important reason for allowing a patient to take part in studies of the antiepileptic properties of new substances. It is usual to invite both men and women to participate in treatment with experimental antiepileptic drugs. Since safety with regard to teratogenic effects is lar less certain for experimental drugs than for established antiepileptic drugs, women must take effective contraceptive precautions and lactating women must be excluded because the test substance secreted with the breast milk will be ingested by the baby. In addition, participants must take their medication consistently and, depending on the purpose of the study, be capable of reporting their seizures and any adverse events. In order that efficacy and adverse events may be 
ascribed to the experimental substance, it is undesirable for the patient to be treated simultaneously with another experimental drug. Serious diseases, especially liver, kidney and cardiovascular diseases, naturally require exclusion of the patient from treatment with an experimental antiepileptic drug because animal and volunteer studies provide only limited information on safety. Progressive diseases such as tumours preclude evaluation of the safety and efficacy of a potential antiepileptic drug, particularly if their progression is rapid, and thus represent grounds for exclusion. Drug and alcohol abusers are regarded as too unreliable in complying with the study design and as too great a health risk to take part.

\subsubsection{Scoring problems and criteria}

The first aim in the treatment of epilepsy is to prevent seizures. In evaluating the effect of a drug that is expected to possess antiepilieptic properties, it is therefore logical to use the number of seizures as a scoring criterion. This poses numerous problems, however: In the first place the patient must be capable of recognizing the seizures himself, or they must be observed by others. If the patient has an aura during seizures, there is a risk that the aura will disappear on treatment with the new drug and the patient will then wrongly believe that he is no longer having seizures. A shift of seizures from day to night is favourable and less stressful for many patients, but impedes observation. The counting of classical absences is difficult, even for experts. Seizures that occur in series must be scored consistently: are seizures that occur in rapid succession to be scored as a single seizure if consciousness does not fully return? Is each seizure of the series counted, or is a series regarded as a different type of seizure (namely "series"), to be scored separately? How rapidly must the seizures follow each other to qualify as a series? And what about the duration of different series of seizures? Is a status epilepticus assigned the same score as an absence of a few seconds? If the patient"s quality of life is examined too, the answer does not seem very obvious. A patient who previously had severe tonicclonic seizures and now, since using the experimental drug, has somewhat more frequent but not very troublesome complex partial seizures shows a deterioration in terms of the number of seizures, even though the patient may be very satisfied with the outcome of the treatment.

Various investigators have proposed that, in addition to counting the number of seizures, a weighting should be assigned to the individual seizures to provide a more precise evaluation of the effect of antiepileptic treatment (Meinardi 1971, Cramer at al. 1983, Baker et al. 1991). In outpatients the problem of scoring the severity and duration of the seizure is greater than in hospitalized patients. A seizure recording system that can be kept accurately and reliably by the patient is therefore required. Such a system does not yet exist. The Chalfont Seizure Severity Scale determines the score of 11 factors: loss of consciousness, warning, dropping of objects held in the hand, falling, injury, incontinence, automatism, convulsions, duration of the seizure, time until normal activities can be resumed (Duncan and Sander 1990). For many patients, particularly for outpatients living alone, the items on this scalle cannot be properly monitored. An alternative endpoint measure is comparison of time to $k$ th seizure. $K$ is predetermined (up to 12) (Shofer and Temkin 1986). Comparing time-to-next seizure before and during treatment with the new drug appears to add information about efficay (Arnoldussen 1989). In an 
endeavour to obtam such a simple method, in this study an attempt was made to weigh the seizure on the basis of the patient"s description (Sections 4.23 and 4.3.3).

\subsubsection{Ethical aspecrs and informed consent}

For each treatment the patient is informed of the nature of the treatment and the associated risks in order to be able to weigh up the expected results and the risk (Dupuis 1988). This information must be given both for conventional drugs and for experimental drugs. Conventional drugs are supplied with a package insert in which the patient can read what the advantages and disadvantages of the treatment are. Package inserts are generally unavailable for experimental drugs because in most cases the available data on the substance is incomplete. Preclinical studies are performed in an attempt to obtain sufficient information on the safety of a drug to permit its administration to a group of selected patients. The investigator and the patient enter into a contract governing treatment with the experimental substance. Both parties must know what they have committed themselves to (Engberts 1988). Accordingly, an informed consent form is drawn up which must state simply and clearly what is required of the patient and what the risks for the patient are (Vermeij 1988). This has already been partly covered in the section on inclusion and exclusion criteria (Section 1.6.1). The patient has the right to withdraw from the study withoul giving reasons. For the purposes of the study, however, it is often useful to know why a patient no longer wishes to participate. For example, if undesirable adverse events have occurred, the implications for the outcome of the treatment are very different than if the patient has moved house and the journey to the investigator has become too llong. The selection of patients takes account of this noncompliance, and this forms a little-reported bias in many studies. The text of the informed consent is assessed by the ethics committee.

\subsubsection{Compliance}

Measurement of compliance is complicated. Regular measurement of plasma concentrations provides some insight into compliance (Meijer 1991). There are factors that impair compliance, such as polyphamacy and the occurrence of adverse reactions (Benet 1990). A simple dosage scheme, on the other hand, improves compliance. In the selection of patients for a study a role is played by the relationship between the investigator and the putient, based on mutual trust. The investigator will select patients whose compliance he expects to be good. This may introduce bias (Pullar et al. 1988). In principle, counting of returned unused medication at each visit tells the investigator how much medication the patient has used, i.e., whether the patient has taken the medication correctly. This method seems rather unreliable, however (Jonkers and Merkus 1978). The drawback of counting in this way is that the patient may feel that the investigator distrusts him. Throughout the studies described in this thesis it was assumed that the patient was taking the medication as instructed by the investigator. By incorporating a preurial period in which the patient has the opportunity to become accustomed to compliance with the study requirements, the reliability of the patient can be verified to some extent. 
Patients who do not respond well to conventional antiepileptic drugs, are selected for studies with new drugs (Section 1.6.1). These are generally patients with a serious, pharmacotherapy-resistant form of epilepsy. Thus it is clear from the outset that few of these patients are likely to respond favourably to the new drug; a modest success may therefore indicate that the drug is particularly effective in other patients. After the open study with loreclezole had taken place (Chapter 3 ) and encouraging results had emerged, a more extensive study of the safety and efficacy of loreclezole was performed to provide more objective data. A double-blind placebo-controlled design was chosen (Chapter 5). As that study too showed that loreclezole was effective and safe, a further study in an even larger number of patients was performed. This phase usually entails add-on treatment. Whether or not loreclezole is also effective as monotherapy can only be discovered after a much longer time; often a drug is only investigated in monotherapy once it has appeared on the market as add-on treatment. This does not alter the fact that in patients from the early study who persisted with the use of loreclezole the comedication was sometimes reduced, with maintenance of the effect or reduction of side effects (Chapter 7). Because patients from the early study are the most resistant to pharmacotherapy, they are also most likely to require persistent polypharmacy. If the comedication can nevertheless be reduced to monotherapy, loreclezole must be regarded as an effective antiepileptic drug. 


\section{Chapter 2. STUDIES WITH R 57720}

$\mathrm{R} 57720$ is an imidazole derivative with anticonvulsant properties as described in Section 1:2 on imidazoles and triazoles. On the basis of animal findings and the volunteer studies to be described below, clinical research in patients with epilepsy was regarded as justified.

Studies in healthy wolunteers showed that R 57720 is absorbed rapidly (Van Peer et al. 1984). The peak blood concentration occurs $45 \mathrm{~min}$ after a single oral dose. The doseconcentration relationship is not linear. Single doses up to $20 \mathrm{mg}$ give a linear relationship between dose and AUC (area under curve) as well as Cmax (maximum plasma concentration). Higher doses up to $80 \mathrm{mg}$ give disproportionate values of AUC and Cmax. The elimination half-life is dose-dependent: low doses up to $20 \mathrm{mg}$ give a half-life of 2$3 \mathrm{~h}$ and higher doses up to $80 \mathrm{mg}$ give a half-life of $4-5 \mathrm{~h}$. Although there are large interindividual differences, it was concluded that nonlinear enzyme kinetics are responsible for the dose-dependency of the half-life that was found. After single doses of 10, 40,80 and $240 \mathrm{mg}$ no side effects were reported by the volunteers. After 1 week of treatment with $40 \mathrm{mg}$ R 57720 there were no changes in biochemical or haematologicall parameters.

\section{$2.2 \quad$ Single dose studies in patients}

A study was conducted into the neurophysiological effects of a single dose of $R 57720$ in patients with photoconvulsive discharges and the effects on the metabolism of the existing antiepileptic drug regimen and on the pharmacokinetics of $R 57720$ itself (Binnie et al. 1985). Single doses of 80,160 and $240 \mathrm{mg} \mathrm{R} 57720$ were given to 4 patients. It was concluded that $80 \mathrm{mg}$ and $160 \mathrm{mg}$ R 57720 suppress the epileptic activity in the EEG of photostimulation-sensitive patients and that there is no interaction with antiepileptic comedication at these doses. This is also true for the studies with $240 \mathrm{mg} \mathrm{R}$ 57720. It was noticeable that the plasma concentration was considerably lower in these patients taking antiepileptic drugs than in volunteers receiving the same dose. The apparent influence of comedication on the plasma level of $\mathbb{R} 57720$ was later confirmed in another study (De Beukelaar et al. 1984). At these dosages plasma half-life times of about $6 \mathrm{~h}$ were found.

Multiple dose studies with ascending doses in patients

Next a safety study was performed in 10 patients with pharmacotherapy-resistant epilepsy (De Beukelaar et al. 1984). These patients were treated for 4 weeks with $40 \mathrm{mg} \mathbb{R}$ 57720 in addition to the existing antiepileptic medication. The EEG, ECG and haematological and biochemical parameters were similar before and after the study. The medication was well tolerated, only one patient reporting a transient rash. No effect on seizure frequency was observed, nor would it be expected at this low dose. On the basis of these findings it was decided to perform an open add-on study with an ascending dosage of $\mathrm{R}$ 57720 in therapy-resistant epilepsy patients. 

"add-on" therapy in therapy-resistant epileptic patients"

This open dose-finding study was designed to evaluate efficacy, safety, the relationship between dose and plasma concentration, and the variability occurring in this relationship. The planned number of patients was 10 .

\subsection{Methods and patients}

\subsubsection{Inclusion and exclusion criteria}

The inclusion criteria for this study were that participants should be epilepsy patients who:

- had not achieved adequate control of their seizures despite optimal therapy with the antiepileptic drugs already prescribed

- suffered from partial seizures in the sense of the international seizure classification, i.e. simple partial, complex partial and partial evolving to tonic-clonic seizures were aged 18 to 60 years

- had given their informed consent to participation in the study

- were inpatients or outpatients prepared to undergo frequent and sometimes stressful investigations

- had been taking stable antiepileptic medication since one month before the study and would continue during the study

- were taking no further medication other than antiepileptic drugs and any oral contraceptives.

The exclusion criteria were:

- elementary partial seizures with only subjective symptoms

- mental retardation and/or diffuse brain damage

- pregnancy or risk of pregnancy

- poor compliance.

Drop-out criteria were:

- withdrawall of consent

- unacceptable changes in biochemical or haematological parameters and/or unacceptable interaction with the existing antiepileptic drugs

- unacceptable side effects

- serious intercurrent disease poor compliance.

\subsubsection{Patients}

Eleven inpatients and outpatients ( 6 women and $5 \mathrm{men}$ ) suffering from partial seizures with complex symptomatology were recruited to the study. Three of them had partial

\footnotetext{
* Investigators in this study were: Hulsman $J$, Declerck $A C$, Janssen $A$, Oet $T$, Rentmeester Th, Startenburg R, Van Ham-Veltman E, De Beukelaar F, Vanden Bussche G.
} 
seizures with secondary generalization (Tables $2.4 . a$ and 2.4 .6 ). In this thesis the 5 parients who subsequently took part in studies with loreclezole can be recognized by their patient identification numbers $(0701,0703,0708,0709$ and 0713). The final two digits can, but do not correspond mecessarilly with the patient identification numbers in the studies with R 57720 . The patients were aged 18 to 40 years (mean 27 years). They had been resistant to pharmacotherapy for 4 to 35 years (mean 15 years). The comedication consisted of carbamazepine ( $5 x)$, phenytoin $(5 x)$, valproate $(4 x)$ and clonazepam (3x). Less common antiepileptic drugs were also prescribed, such as oxcarbazepine (1 $x)$, acetazolamide $(1 x)$, clobazam $(2 x)$, and clonazepam $(3 x)$. There were no patients

\begin{tabular}{|llllll|}
\hline pt & sf/4 weeks & sz.type & AED & age (yrs) & sex \\
10 & 7 & c.p. & CBZ, PHT & 21 & $\mathrm{~F}$ \\
4 & 8 & c.p. ${ }^{*}$ & OXC, CLN & 19 & $\mathrm{M}$ \\
6 & 11 & c.p. & CBZ, VPA & 18 & $\mathrm{~F}$ \\
7 & 12 & c.p. & CBZ, AZA & 24 & $\mathrm{~F}$ \\
0713 & 12 & c.p. & CBZ, CLN & 28 & $\mathrm{~F}$ \\
0701 & 12 & c.p.* & CBZ, VPA, CLB & 35 & $\mathrm{M}$ \\
0709 & 12 & c.p. & PHT, VPA & 40 & $\mathrm{~F}$ \\
0703 & 20 & c.p. & CBZ, VPA & 23 & $\mathrm{M}$ \\
2 & 20 & c.p. & CBZ, PHT, PB, CLN & 28 & $\mathrm{~F}$ \\
0708 & 20 & c.p.* & CBZ, PHT & 34 & $\mathrm{M}$ \\
8 & 108 & c.p. & CBZ, PHT, CLB & 28 & $\mathrm{M}$ \\
\hline
\end{tabular}

Table 2.4.a. Patient characteristics (1): $C B Z=$ carbamazepine, $P H T=$ phenytoin, $V P A=$ valproate, $O X C=$ axcarbazepine,$C L B=$ clabazam, $C L N=$ clonazepam, $A Z A=$ acerazolanide: $P B=$ phenobarbital, c.p. = complex partial seizure, sztype = seizure type, * = secondarily generalized. $s f / 4$ weeks $=$ seizure frequency per 4 weeks

\begin{tabular}{|llllll|}
\hline pt & $\begin{array}{l}\text { age of } \\
\text { onset (yrs) }\end{array}$ & dur.th.rst & etiology & $\begin{array}{l}\text { height } \\
(\mathrm{cm})\end{array}$ & $\begin{array}{l}\text { weight } \\
(\mathrm{kg})\end{array}$ \\
10 & 10 & 11 & unknown & 165 & 55 \\
4 & 9 & 9 & unknown & 176 & 70 \\
6 & 2 & 16 & birth tr. & 160 & 67 \\
7 & 20 & 4 & unknown & 157 & 53 \\
0713 & 12 & 16 & unknown & 166 & 60 \\
0701 & 0 & 35 & birth tr. & 175 & 75 \\
0709 & 8 & 32 & birth tr. & 163 & 83 \\
0703 & 19 & 4 & cer.conc. & 184 & 80 \\
2 & 13 & 13 & unknown & 155 & 66 \\
0708 & 11 & 23 & unknown & 174 & 80 \\
8 & 14 & 14 & unknown & 176 & 66 \\
\hline
\end{tabular}

Table 2.4.b. Patient characteristics (2), dur.th.rst. = dwation of therapy resistance, birth tr. $=$ birth wawn, cerconc. = cerebral concussion 


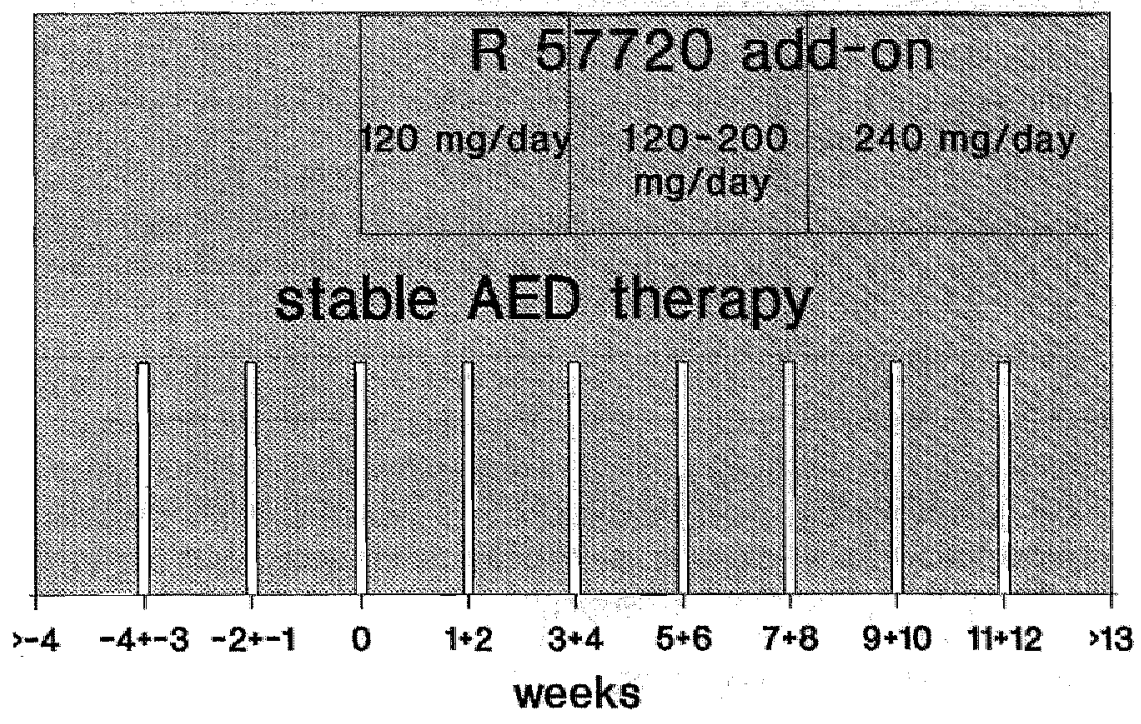

Fig. 2.4.c. Study design. $R 57720$ add-on study on safery and efficacy. Evaluation every 2 weeks.

- plasma concentrations of R 57720 and other antiepileptic drugs, including metabolites, immediately after the first dose of the day

- haematological investigations: red and white blood cells, platelets, haemoglobin, haematocrit, sedimentation, differential blood count

- biochemical investigations: glucose, urea, creatinine, bilirubin, ASAT, ALAT, $\gamma$-GT, alkaline phosphatase, sodium, potassium, calcium, chloride, phosphate, total protein

Table 2.4.d. Laborahory determination.

with monotherapy; 8 patients took 2, I. patient took 3 and 2 patients took 4 antiepileptic drugs. After a 4-week pretrial period in which the seizure frequency was 7 -108 seizures per 4 weeks (mean $12 / 4$ weeks), R 57720 was added to the existing medication. On the account of the plasma half-life time of about $6 \mathrm{~h}$ R 57720 was administered 3 times per day in the form of capsules. After each 4-week period while $R 57720$ was being administered, a decision was made with regard to further treatment; this is shown in the study design (Fig. 2.4.c). The seizure frequency and any adverse events were evaluated after each period. If the reduction of the seizure frequency was insufficient and $R 57720$ was well tolerated the initial dose of $120 \mathrm{mg}$ ( 3 times $40 \mathrm{mg} /$ day) was raised to 160 or 200 $\mathrm{mg} / \mathrm{day}$, depending on tolerance, efficacy, interaction with comedication and changes in labarotory data. These criteria also applied to the third 4-week phase in which the medication was raised to $240 \mathrm{mg} /$ day. Every 2 weeks the haematological and biochemical parameters and plasma concentration of AED's were determined before the first dose of 
the day (Table 2.4.d). At the end of the trial the participating palients were given the opportunity to continue $\mathrm{R} 57720$ treatment if the patient derived benefit from the drug.

\section{$2.4 .2 \quad$ Results}

\subsubsection{Seizure frequency}

As stated above, at the end of each 4-week phase a decision was made regarding further treament (in particular the $\mathbb{R} 57720$ dosage) in the following 4 weeks. In phase 1 (in which the $R 57720$ dose was $120 \mathrm{mg} /$ day) the seizure frequency was 4 to 43 per 4 weeks (mean $13.5 / 4$ weeks) $(\mathrm{p}=0.44)$; in 4 of the 11 patients the seizure frequency fell by more than $50 \%$. In phase 2 (160 to $200 \mathrm{mg}$ R 57720 day) the serzure frequency was 1 to 25 seizures per 4 weeks (mean 13 seizures per 4 weeks) $(p=0,30)$; with respect to the pretrial period there was a seizure reduction of $50 \%$ in a total of 3 of the 10 remaining patients ( 1 patient had dropped out on account of rash). In the third phase (in which the maximum dose of $240 \mathrm{mg} / \mathrm{day}$ was given) the seizure frequency was 1 to 13 seizures per 4 weeks (mean 7.3 seizures per 4 weeks) $(p=0.008)$; there was a reduction of more than $50 \%$ in a total of 5 of the 10 patients (Wilcoxon matched pairs-signed rank test, two-tailed) (Figs. 2.4.e, 2.4.f, and 2.4.g).

In Figs. 2.4.e, 2.4.f and 2.4.g the seizure frequency per 4 weeks is plotted on the vertical axis. The horizontal axis in Fig. 2.4.e gives the identity of the patient (patients who later took loreclezole are identified by a number beginning with 07 ), the study phases being plotted from left to right (weeks $1-4,5-8$ and 9-12). Fig. 2.4.g shows that, on average, a higher plasma concentration of $\mathrm{R} 57720$ is associated with better seizure control, a reduction in frequency was achieved not only in patients with a very high seizure frequency but also in patients whose initial seizure frequency was already fairly low. Patient 0713 experienced a obvious reduction in seizure severity, without reduction in seizure frequency. Of the 10 patients who completed the study, 3 stopped using $R$ 57720 on account of insufficient motivation to continue the investigation (patient 2 ) or insufficient effect on seizure frequency (patients 4 and 0703).

\subsubsection{Adverse events}

The R 57720 medication was tolerated well by all patients except 1 (patient 10) who cleveloped a generalized papulous exanthem aften 14 days. The rash disappeared rapidly on withdrawal of $\mathrm{R} 57720$. Two patients reported mild pyrosis during the study, but this did not necessitate withdrawal of the treatment. In 1 patient (patient 0709) the plasma concentration of comedication (phenytoin) rose (with intoxication symptoms), so that adaptation (lowering of the phenytoin dose) was necessary, whereupon the phenytoin concentration nomalized and the symptoms disappeared.

In 4 patients $\gamma$-GT was elevated (up to $60 \mathrm{U} / 1$, normal range up to $30 \mathrm{U} / 1$ ) in the pretrial period, while alkaline phosphatase, ALAT, ASAT and bilirubin remained within the normal limits in these 4 patients (patients 2, 0708, 0709 and 6) (Table 2.4.h). During the study no patient showed any significant changes in the biochemical and haematological parameters. 


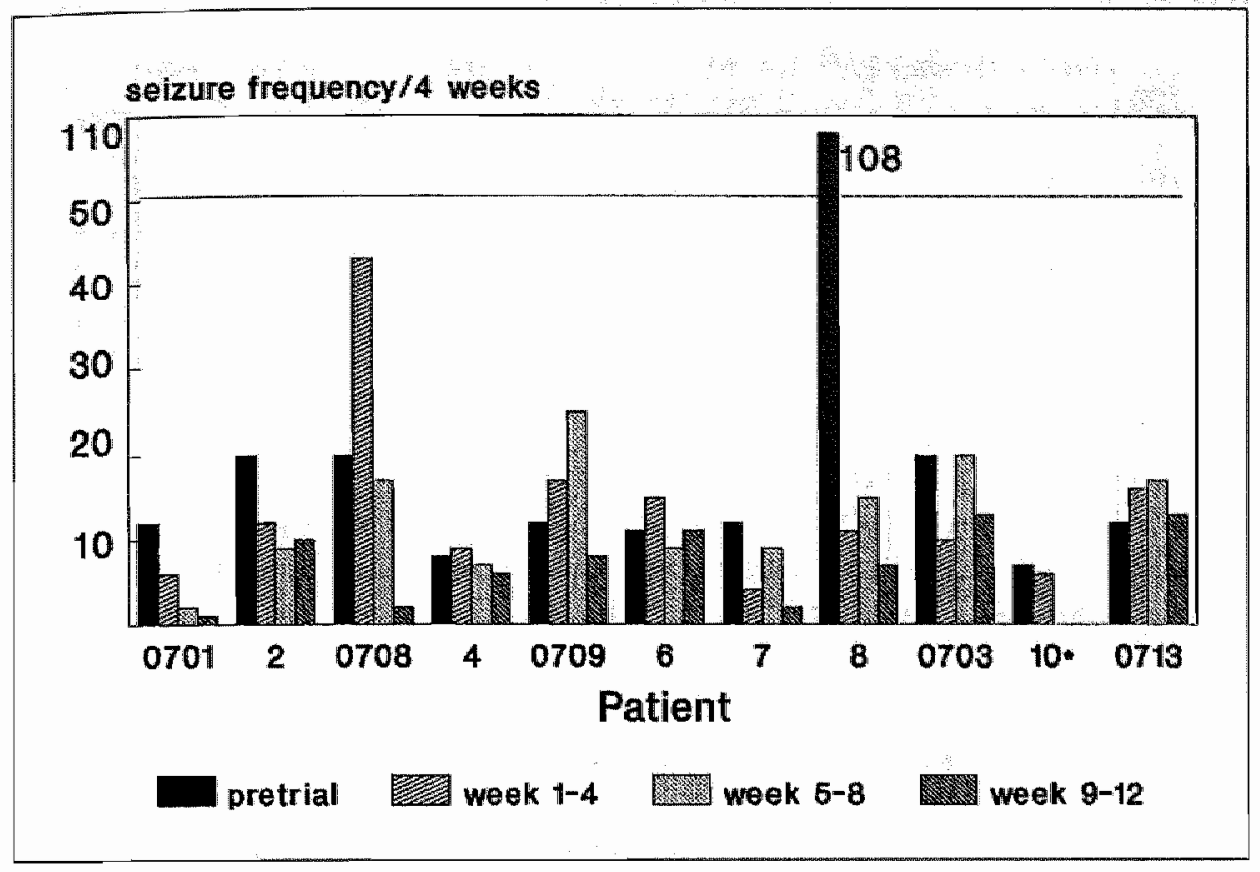

Fig. 2.4.e. Efficacy of $R$ 57720. Seizure frequency/4 weeks in treatment with $R 57720$ as addon therapy.

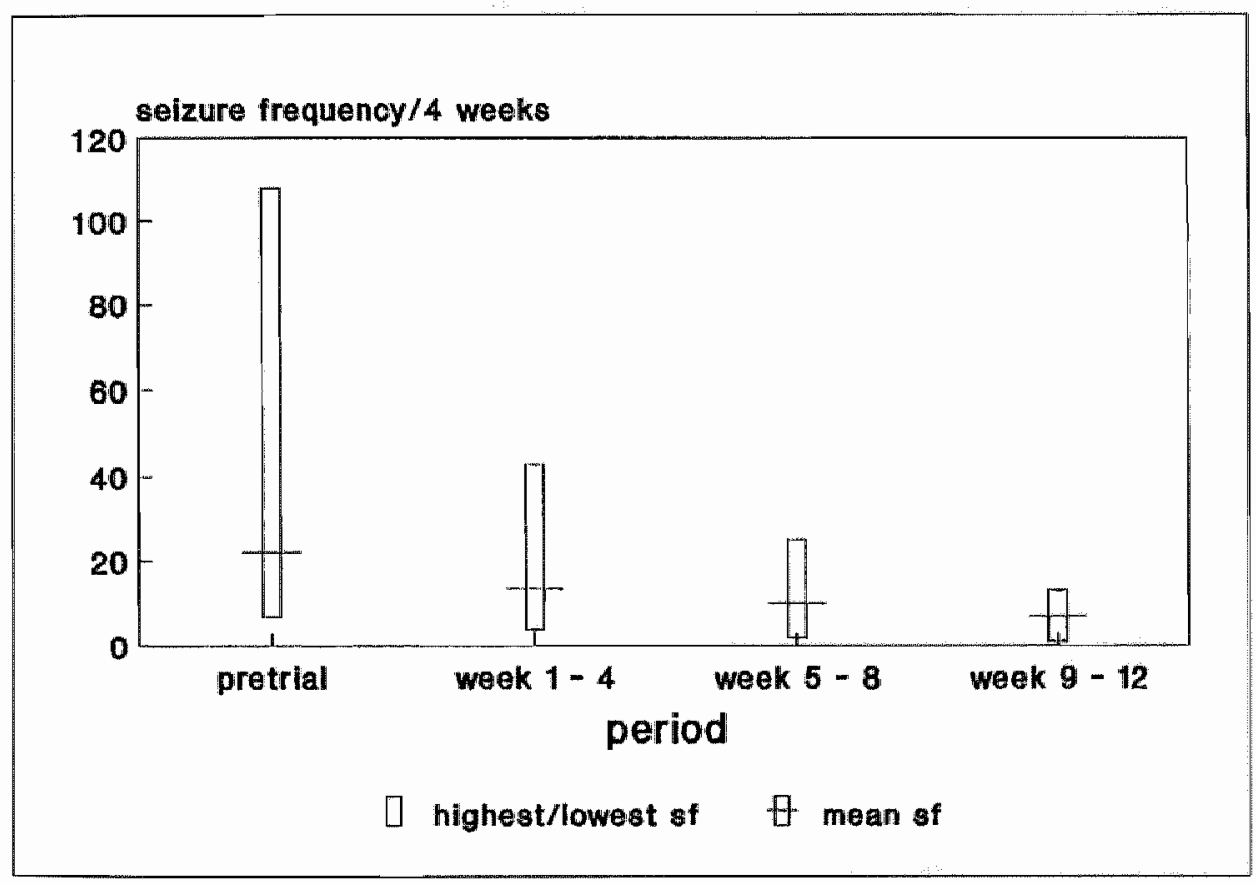

Fig. 2.4.f. Seizure frequency (sf) rate. $R 57720$ as add-on the rapy. 


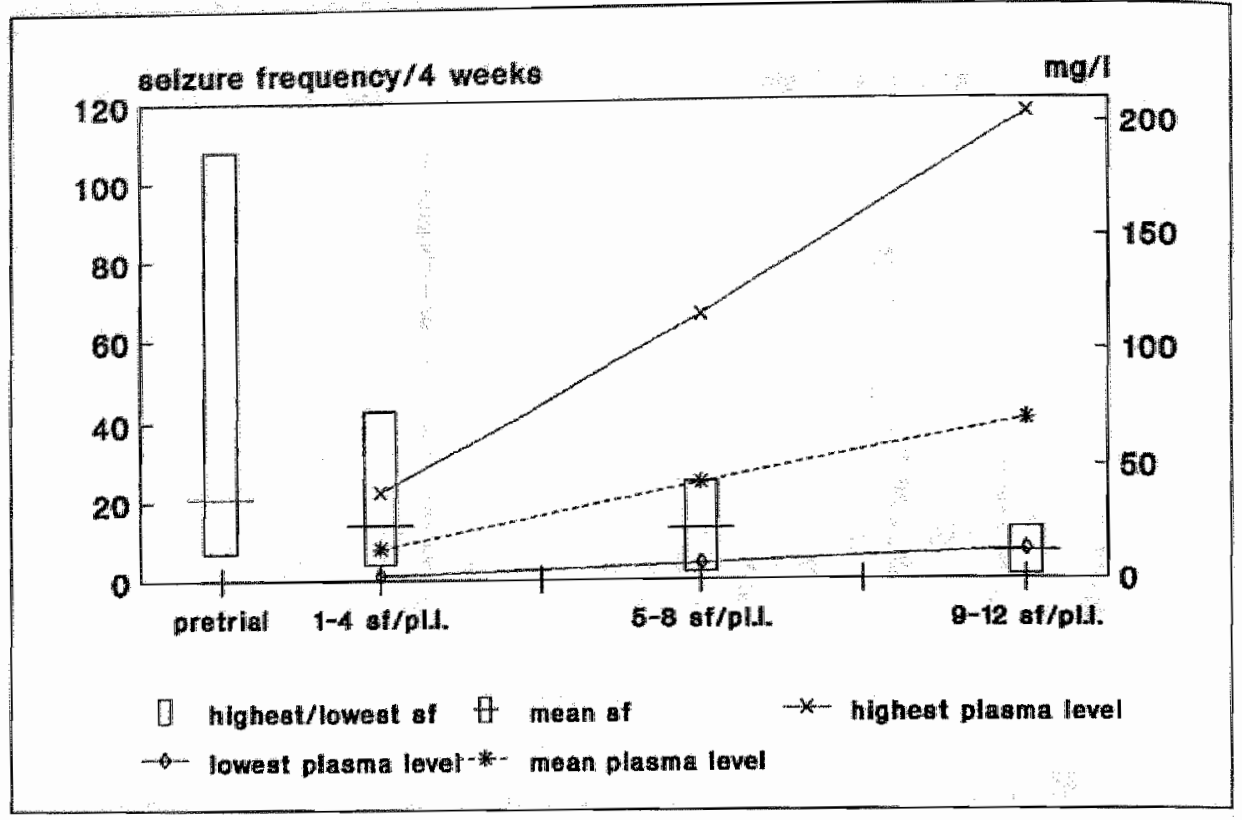

Fig. 2.4.g. Setzure frequency (sf) ws. plasma concentrations of $R 57720$.

bar $=$ seizure frequency/4 weeks line = plasma concentration (pl.c.)

\subsubsection{Follow-up}

Seven patients continued the medication because the treatment was considered to be successful. Two patients continued the R 57720 treatment for a good 2 years. When they stopped after these 2 years (because no more substance was available) the seizures returned immediately in one of them (patient 0701) while the other remained seizurefree for some months. In 1 patient the seizures returned after 3 months despite continuation of the $R 57720$ medication. The patient consequently stopped the R 57720 treatment. One other patient was not motivated to continue treatment with $R 57720$. In 3 patients (patients 0708, 0709 and 0713) hepatotoxic signs appeared after 4 months of use: elevation of $\gamma$ GT to $87 \mathrm{U} / \mathrm{L}$, ALAT to $196 \mathrm{U} / \mathrm{h}$ (normal range up to $35 \mathrm{U} / \mathrm{l}$ ) and ASAT to $110 \mathrm{U} / 1$ (normal range up to $25 \mathrm{U} / 1$ ). Two patients (patients 0708 and 0709 ) had already had an elevated $\gamma$ GT (to $43 \mathrm{U} / \mathrm{l}$ ) in the pretrial period. During the hepatotoxicity phase, evident clinical symptoms (general malaise, extreme tiredness, nausea and vomiting) appeared in patient 0709. One patient (patient 0708) complained of vague malaise and 1 patient (patient 0713 ) remained asymptomatic despite the abnomal laboratory findings. Nevertheless, the hepatotoxic phenomena led to withdrawal of R 57720 in all 3 patients. In all 3 these parameters normalized after a few days, i.e. after the period that would be expected on the basis of the half-life of R 57720 . In all 3 of these patients the seizures returned to their original frequency after withdrawal of the $R$ 57720 . In patient 0709 the $\mathrm{R} 57720$ had had a favourable effect on the frequency of seizures, which relspsed after its withdrawal, and so $R 57720$ treatment was resumed. Almost immediately the clinical symptoms and chemical findings of hepatotoxicity returned, confirming the relationship between these phenomena and the use of $R 57720$. The use of R 57720 was subsequently discontinued. 


\begin{tabular}{|c|c|c|c|c|c|}
\hline pt & $\gamma \mathrm{GT}$ & $\mathrm{AF}$ & ALAT & $\mathrm{ASAT}$ & bili \\
\hline 0701. & $19 / 33$ & $24 / 24$ & $12 / 15$ & $12 / 16$ & $7.6 / 82$ \\
\hline 2 & $59 / 74$ & $62 / 60$ & $20 / 36$ & $13 / 20$ & $7.0 / 4.1$ \\
\hline 0708 & $52 / 87$ & $76 / 74$ & $24 / 58$ & $18 / 36$ & $7.6 / 7.3$ \\
\hline 4 & $19 / 20$ & $92 / 79$ & $17 / 12$ & $13 / 15$ & $6.1 / 9.7$ \\
\hline 0709 & $43 / 54$ & $98 / 94$ & $10 / 47$ & $8 / 32$ & $7.2 / 5.3$ \\
\hline 6 & $36 / 28$ & $40 / 36$ & $12 / 7$ & $10 / 8$ & $8.2 / 7.7$ \\
\hline 7 & $24 / 30$ & $44 / 61$ & $14 / 11$ & $10 / 7$ & $11.6 / 4.4$ \\
\hline 8 & $21 / 23$ & $84 / 97$ & $7 / 6$ & $8 / 9$ & $11.2 / 12.9$ \\
\hline 0703 & $12 / 24$ & $60 / 89$ & $17 / 16$ & $25 / 16$ & $10.7 / 10.2$ \\
\hline $10^{*}$ & $90 / 90$ & $88 / 90$ & $28 / 20$ & $20 / 12$ & $5.9 / 8.0$ \\
\hline 0713 & $24 / 38$ & $42 / 42$ & $8 / 12$ & $8 / 8$ & $5.9 / 5.6$ \\
\hline \multicolumn{6}{|c|}{ *Determination on day 15 , drop-out } \\
\hline pt & $\gamma-\mathrm{GT}$ & $\mathrm{AF}$ & ALAT & ASAT & bili \\
\hline 0701 & 21 & 23 & 16 & 17 & 8.0 \\
\hline 0708 & 87 & 70 & 134 & 55 & 8.5 \\
\hline 0709 & 69 & 100 & 196 & 110 & 8.0 \\
\hline 0703 & 26 & 103 & 8 & 9 & 4.1 \\
\hline 0713 & 70 & 42 & 96 & 35 & 8.2 \\
\hline \multicolumn{6}{|c|}{ Determination 120.} \\
\hline
\end{tabular}

Table: 2.4.h. Biochemical parameters pretrial/on day 85 and on day $120:$ pretrial; $\gamma$ GT, alkaline phosphatase, ALAT, ASAT, and bilirubin. Bold = abnormal value.

The 2 patients (patients 0701 and 8 ) in whom R 57720 also had a very favourable therapeutic effect and who had no adverse events continued the R 57720 treatment as long as the supply lasted (a good 2 years). In one of them (patient 0701) $\gamma$-GT had been elevated before administration of R 57720. Both patients showed no clinical or biochemical signs of toxicity at any stage in the R 57720 treatment. In patient 0701 the seizures relapsed rapidly after withdrawal of $R 57720$.

\subsubsection{Discussion}

The imidazole derivative $\mathbb{R} 57720$ appeared to be an effective antiepileptic drug, with dose-dependent activity in 7 of 11 therapy-resistant patients. One patient dropped out on account of rash, attributed to an allergic reaction. Two patients reported adverse events in the form of mild pyrosis, possibly due to $\mathrm{R} 57720$. This did not necessitate their withdrawal from the study. The seizure severity did not worsen in any of the patients. Four of the 11 patients showed a considerable (greater than $50 \%$ ) reduction in seizures (which however failed to persist in 1 patient) at a dose of $120 \mathrm{mg} /$ day. At the maximum final dose of $240 \mathrm{mg} /$ day, 5 of the 10 patients had a seizure reduction of more than $50 \%$ 
(Fig. 2.4.). The mean seizure frequency fell from 23 seizures per 4 weeks in the pretrial period to 7 seizures per 4 weeks in the third phase. There was no difference in treatment of responders and nonresponders. The mean $R 57720$ dose did not differ substanhally between the responders and the nomesponders: $1.7,2.3$ and $3.5 \mathrm{mg} / \mathrm{kg} /$ day in the respective periods. A better mean effect was observed as the plasma level rose, however (fig. $2.4 \mathrm{~g})$. In 1 patient the phenytoin comedication was modified on account of intoxication symptoms ascribed to an increase in the plasma phenytoin concentration. After reduction of the phenytoin dosage to give the origiral plasma concentration the seizure control persisted; from which it must be concluded that the favourable effect was due to the $\mathbb{R} 57720$ medication. As the R 57720 treatment continued, 3 patients (of whom 1 , as stated above, had had elevated $\gamma$-GT in the pretrial period and another had had a borderline value) showed serious elevation of the enzymes ALAT and ASAT, whilst bilirubin remained normal. Two of these 3 patients also showed clinical signs of hepatotoxicity. All 3 patients recovered immediately, within the expected time, after withdrawal of $R$ 57720 . In all 3 the seizures returned within a few days, at the same frequency as before the $\mathrm{R} 57720$ treatment.

\subsubsection{Conclusion}

From the studies that have been performed, R 57720 seems to be an effective antiepileptic drug, both in animal studies and in patients with serious partial epilepsy. When administered for a short period, the substance had no substantial side effects - apart from possible hypersensitivity reactions - and no influence on biochemical and haematological parameters. A conspicuous finding in the open add-on trial is the onset of evident hepatotoxic signs in 3 responders after treatment for more than 4 months. In the pretrial phase these 3 patients had had high normal or slightly elevated $\gamma-G T$ walues. It would be wrong to infer that this might have some predictive value for the onset of a hepatotoxic reaction, however, particularly since $\mathbb{1}$ patient whose $\gamma$-GT was elevated before the R 57720 treatment tolerated the substance well for a long period. On withdrawal of $R 57720$ the enzymes normalized rapidly. The immediate return of the hepatotoxic signs alter reintroduction of R 57720 in one of the patients shows that a relationship between these disturbances and the use of $R 57720$ is very probable. In 1 patient the seizures relapsed despite continued use of $R 57720$. Two patients used R 57720 for 2 years and remained virtually free of seizures, with no signs of hepatotoxicity. These favourable results provided the motivation for further research into substances from the imidazole and triazole groups which have a stucture similar to $\mathrm{R} 57720$ but which are likely to be less hepatotoxic and may therefore form the basis for development of a new generation of antiepileptic drugs. The triazole loreclezole was chosen and studies with this compound are described in the next chapters. 


\section{Chapter 3. OPEN STUDY OF THE EFFICACY AND SAFETY OF \\ LORECLEZOLE AS ADD-ON THERAPY IN THERAPY-RESISTANT EPILEPSY PATIENTS*}

Introduction

The aim of this study was to investigate the safety, efficacy and side effects of loreclezole given as long-term antiepileptic treatment. In the first instance the kinetics of a single loreclezole dose were investigated. The findings with regard to half-life, maximum plasma concentration and interaction with comedication provided the starting point for establishing a dosage scheme for long-term loreclezole treatment. On the basis of data that were already available from volunteer studies and from other investigations (Section 1.5.2), the dose was set at a concentration equal to one twentieth to one tenth of the toxic dose in experimental animals and equal to the dose that was well tolerated by volunteers, giving a plasma loreclezole concentration of $1-2 \mathrm{mg} / \mathrm{l}$ with a maximum of 3 $\mathrm{mg} /$, which on the basis of the animal studies was expected to prove effective. Thirteen patients were treated in the open mode for 6 months.

\section{2}

\section{Methods and patients}

\section{2 .1}

\section{Inclusion and exclusion criteria}

The inclusion criteria were:

- well defined pharmacotherapy-resistant epilepsy

- despite optimal antiepileptic drug therapy at least 4 seizures per month

- age 17 - 60 years

- stable antiepileptic drug regimen since one month before the study and during the study

- given informed consent to participation in the study.

The exclusion criteria were:

- patients with serious or progressive liver or kidney dysfunction

- patients with progressive neurological abnormalities

- insufficient compliance

- mentall deficiency to the extent that assessment of treatment results is inadecuate

- risk of pregnancy

- use of comedication other than antiepileptic drugs or contraceptives

- recent participation in a study with another experimental drug, except $\mathrm{R} 57720$ (Section 2.4)

- withdrawal of consent.

* The resulss of this study are published as: Rentmeester T, Hulsman J. Efficacy and safety evaluation of loreclezole as add-sn treatment in the rapy-resistent epilepsy patients. Epilepsy Res 1991; 8:166-169 
Thirteen pharmacotherapy-resistant patients with 4 or more seizures per month were selected. Both inpatients and outpatients were included. One patient had symptomatic generalized epilepsy and 12 had epilepsy manifested as partial seizures with complex

\begin{tabular}{|lrllll|}
\hline pt & sfl/ weeks & sz.type & AED & age (yrs) & sex \\
0701 & 24 & c.p. & CBZ, VPA, CLZ & 37 & $\mathrm{M}$ \\
0702 & 8 & c.p.* & CBZ, VPA, AZA & 38 & $\mathrm{~F}$ \\
0703 & 49 & c.p. & PHT, VPA & 26 & $\mathrm{M}$ \\
0704 & 22 & c.p.* & CBZ, AZA & 20 & $\mathrm{~F}$ \\
0705 & 6 & c.p.* & CBZ & 52 & $\mathrm{M}$ \\
0706 & 24 & c.p.* & CBZ, PRM & 36 & $\mathrm{M}$ \\
0707 & 29 & c.p. & OXC, PRM & 30 & $\mathrm{~F}$ \\
0708 & 16 & c.p. & PHT, AZA & 37 & $\mathrm{M}$ \\
0709 & 44 & c.p. & PHT & 44 & $\mathrm{~F}$ \\
0710 & 62 & c.p.* & CBZ, VPA & 41 & $\mathrm{M}$ \\
0711 & 17 & c.p.* & CBZ, CLB & 42 & $\mathrm{M}$ \\
0712 & 7 & c.p.* & CBZ, PHT & 31 & $\mathrm{~F}$ \\
0713 & 7 & c.p. & CBZ, CLN, AZA & 31 & $\mathrm{~F}$ \\
& & & & & \\
\hline
\end{tabular}

Table 3.2.a. Patient characteristics (1). CBZ $=$ carbamazepine, $P H T=$ phenytoin, $V P A=$ valprow ate, $O X C=$ axcarbazepine,$C L B=$ clobazam, $C L N=$ clonazepam, $C L Z=$ clorazepate,$A Z A$ $=$ acetazolamide, $P B=$ phenobarbital, $c . p=$ complex partial seizure, sz type $=$ seizure type, * secondarily generalization, sf/4 weeks = seizure frequency/4 weeks

\begin{tabular}{|lcrlll|}
\hline pt & $\begin{array}{l}\text { age of onset } \\
\text { (yrs) }\end{array}$ & dur.th.rst. etiology & height $(\mathrm{cm})$ & weight(kg) \\
& & & & & \\
0701 & 8 & 29 & unknown & 178 & 78 \\
0702 & 10 & 27 & unknown & 167 & 72 \\
0703 & 20 & 6 & cer.conc. & 184 & 80 \\
0704 & 11 & 9 & A.V.M. & 165 & 60 \\
0705 & 45 & 7 & unknown & 168 & 60 \\
0706 & 16 & 20 & br.abscess & 181 & 79 \\
0707 & 14 & 16 & unknown & 172 & 49 \\
0708 & 11 & 17 & unknown & 174 & 79 \\
0709 & 8 & 36 & unknown & 163 & 62 \\
0710 & 25 & 10 & birtla tr. & 181 & 83 \\
0711 & 8 & 34 & unknown & 193 & 83 \\
0712 & 6 & 18 & enceph. & 175 & 73 \\
0713 & 12 & 19 & unknown & 171 & 56 \\
& & & & & \\
\hline
\end{tabular}

Table 3.2.b. Patient characteristics (2). durth.rst $=$ duration of therapy resistance, cer.conc $=$ cerebral concussion, birth $t r .=$ birth trauma, brabscess = brain abscess, enceph = encephalitis 
symptomatology, some with secondary generalization (Table 3.2.a and 3.2.6). Age ranged from 20 to 52 years (mean 37 years); duration of epilepsy ranged from 6 to 36 years (mean 20 years). Six women and 7 men took part in the study. Five of the participants had previously used R 57720 (patients 0701, 0703,0708,0709 and 0713). Patient 0701 had actually been taking R 57720 until shortly before the study, and had been virtually free of seizures as a result. As R 57720 was no longer available, that treatment had come to an end and the seizures had retumed, with the same frequency as before the use of R 57720 . Patient 0703 had stopped using R 57720 owing to inefficacy. Patients 0708,0709 and 0713 had long since stopped using R 57720 on account of hepatotoxic symptoms (Section 2.4).

All patients were taking antiepileptic drugs, some in combination with oral contraceptives, but no other medication; during the study, incidental medication was avoided wherever possible. Two patients were on monotherapy, 8 were taking 2 antiepileptic dirugs and 3 were taking 3 antiepileptic drugs. Nine were taking carbamazepine, 4 phenytoin, 4 valproate, 2 primidone, 4 acetazolamide, 1 clobazam, 1 clonazepam, 1 clorazepate and 1 oxcarbazepine (although this drug had still not been officially registered in the Netherlands when the loreclezole study was started, in view of the long-standing familiarity with oxcarbazepine in the epilepsy centres it was not regarded as an investigational drug). During the study the dosage scheme of the comedication was kept constant unless side effects occurred, and the patient was asked to keep an accurate record of seizures and adverse events. The study design is shown schematically in Fig. 3.2.c. Loreclezole was given in capsules. As regards the existing medication, blood samples were taken every $2 \mathrm{~h}$ from 8.00 a.m. (before the morning dose) to 6.00 p.m. on one day

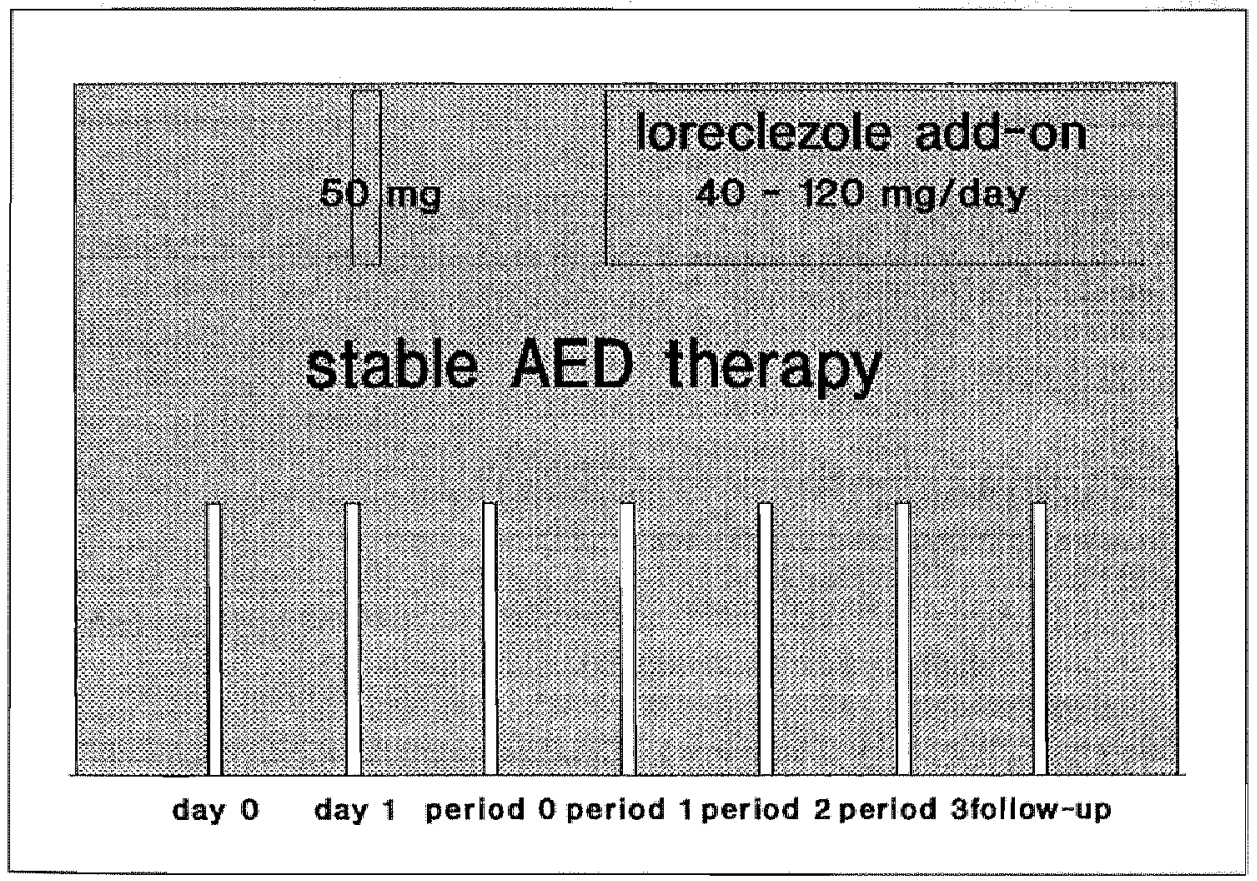

Fig. 3.2.c. Siudy design of the open add-on study on safety and efficacy of loreclezole. 
- plasma concentrations of loreclezole and other antiepileptic drugs, including metabolites and any free fractions

- haematological investigations: red and white blood cells, platelets, haemoglobin, haematocrit, sedimentation, differential blood count

- biochemical investigations: glucose, urea, creatinine, uric acid, total and direct bilirubin, ASAT, ALAT, $\gamma$-GT, alkaline phosphatase, LDH, cholesterol, sodium, potassium, calcium, chloride, phosphate, total protein, albumin

Table 3.2.d. Laboratory determination for the study with a single dose of loreclezole.

(= day 0) to determine various haematological and biochemical parameters and the plasma concentration of the antiepileptic drugs used (Table $3.2 \mathrm{~d}$ ). On day 1 the same investigation was carried out, but immediately after the 8.00 a.m. blood sampling $50 \mathrm{mg}$ loreclezole was given orally. Single blood samples were taken on days $2,3,8,15$ and 22 . The data from this study were to be used to calculate the dose for chronic loreclezole treatment, in which a plasma concentration of 1-2 $\mathrm{mg} / 1$ was targeted. Loreclezole was given once per day. As mentioned in the Introduction, the plasma concentration of 1-2 mg/l was chosen on account of its efficacy in animal studies (Section 1.2).

The patients were evaluated neurologically at intervals of no more than 1 month. In the first instance the dose was adjusted to give a plasma concentration of approximately 1 $\mathrm{mg} / \mathrm{l}$. If this gave insufficient effect and there were no side effects the loreclezole dose was raised to a plasma concentration of $2 \mathrm{mg} / \mathrm{l}$ and, if necessary, to a maximum of about $3 \mathrm{mg} / \mathrm{l}$. In the event of side effects the dose was reduced to the previous level which had been well tolerated. If the plasma concentration of the comedication moved outside the patient's usual range, the comedication dose was adjusted to restore the initial plasma concentration. During the first 4 months of the study the biochemical and haematological parameters were checked on a limited scale every 14 days and comprehensively every month. Plasma concentrations of the antiepileptic drugs were checked every 2 weeks, before ingestion of the medication (Table 3.2.e).

- plasma concentrations of loreclezole and other antiepileptic drugs, including metabolites and any free fractions, immediately after the first dose of the day

- haematological investigations: red and white blood cells, platelets, haemoglobin, haematocrit, sedimentation, differential blood count

- biochemical investigations: glucose*, urea*, creatinine* , bilirubin, ASAT, ALAT, $\gamma$-GT, alkaline phosphatase, sodium*, potassium* ${ }^{*}$, calcium*, chloride*, phosphate*, total protein*.

Table 3.2.e. Laboratory determination for the open add-on study with chronic administration of loreclezole ${ }^{*}=$ determined only in the comprehensive investigations). 
In all studies described in this thesis loreclezole plasma concentrations were determined by HPLC.

\subsubsection{Procedure}

To a glass-stoppered tube $10 \mu \mathrm{l}$ internal standard diazepam is transferred with a microlitre syringe (Hamilton $701 \mathrm{NWG} 10 \mu \mathrm{l}$ ) and $400 \mu \mathrm{l} 0.1 \mathrm{~N}$ sodium hydroxide solution is added with a dispenser (Nichiryo model 8100). Then $500 \mu \mathrm{l}$ plasma is pipetted (Oxford no 3004 , adjustable from $200-1000 \mu \mathrm{L}$ ). The sample is stored till analysis at $-20^{\circ} \mathrm{C}$. After defrosting, the sample is thoroughly mixed (Eckli type 600). Eight $\mathrm{ml}$ dichloromethane (Baker 9315 Baker Analyzed HPLC Reagent) is added with a dilutor (Oxford dillutor model 450). Then close the tube with a glass stopper and shake well for 10 minutes (Infors AG type TU-1, 111 shakings per minute). Next the tube is centrifuged with a thermostatic centrifuge (Hettich 4200 Rotixa RP) at 4000rpm (corresponding with $3400 \times \mathrm{g}$ ) at $20^{\circ} \mathrm{C}$ for 10 minutes. The aqueous layer is aspirated and discarded by means of a Pasteur pipette (WU Mainz $150 \mathrm{~mm}$ ) connected to a water jet pump (Haake NP $631 / 2$ ). The tubes are placed in a thermostat (Thermochem type $\mathrm{CH} 20$ ) and the organic layer is evaporated to dryness at $40^{\circ} \mathrm{C}$ in nitrogen (HoekLoos S 70 nitrogen 3.0 technical). After evaporation the tubes are immediately transferred from the metal block thermostat. The residue is diluted in $200 \mu \mathrm{l}$ Mobile Phase (62\% water, $38 \%$ acetonitrile) and after intensive mixing (Eckli type 600) the solution is transferred to a $200 \mu \mathrm{l}$ vial (Chromacol vial 03-CVG) and capped (Chromacol Cap/seal 8-AC-TST1) with a capitator (Wheaton type 224300 Millville, N.J.). Samples of $20 \mu \mathrm{l}$ are injected on a liquid chromatograph.

\section{Materials}

* $0.1 \mathrm{~N} \mathrm{NaOH}$ Baker 4687 Dillut-it sodium hydroxide $0.1 \mathrm{~mol} / \mathrm{l}$

* water, for HPLC (Baker 4218)

Baker Analyzed HPLC Reagent.

* acetonitrile, for HPLC (Baker 8143)

Baker Analyzed HPLC Reagent.

\subsubsection{Chromatography}

The HPLC system consisted of an HP 1090 LUSI series II with a diode array detector, an automatic injection system, a heating system (Hewlett Packard) and an integrator (HP 3396 A Hewlett Packard). The separation is done at $50^{\circ} \mathrm{C}$ on a $100 \times 3.0 \mathrm{~mm}$ (I.D.) prepacked chromsphere $\mathrm{C} 18$ cartridge column (particle size $5 \mu \mathrm{m}$ ) (Chrompack 28267). The mobile phase consists of a mixture of $620 \mathrm{ml}$ water (Baker 4218) and $380 \mathrm{ml}$ acetonitrile (Baker 8143). After mixing, the solution is filltrated in a vacuum filtration apparatus (Schleicher and Schuell Selectron) with a membrane filter (Schleicher und Schuell membrane filter RC $550.45 \mu \mathrm{m} 50 \mathrm{~mm}$ ). First $\mathrm{S} 70$ nitrogen is led over a wash bottle fil led with Molecular Sieve $5 \mathrm{~A}^{\circ}$ 1/16 pellets. Degasing is done with helium (HoekLoos E 70). 


\section{Conditions:}

flow: $0.80 \mathrm{~m} / \mathrm{min}$

* heating column compartment: $50^{\circ} \mathrm{C}$

* injection volume: $20 \mu 1$

* run time: 6.70 minutes

wavelength: $230 \mathrm{~nm}$

Under these chromatographical conditions loreclezole is separated from other antiepileptic drugs and from the internal standard (diazepain).

\subsection{Results of the single dose study}

The results obtaned from the single dose study are given in Table 3.3.a.

\subsubsection{Plasma concentrations}

In this study a single oral dose of $50 \mathrm{mg}$ loreclezole was given. After $2-5 \mathrm{~h}$, maximum plasma loreclezole concentrations of $0.17-0.51 \mathrm{mg} / \mathrm{l}$ (mean $0.36 \mathrm{mg} / \mathrm{l}$ ) were found, which served as starting values for calculating the appropriate loreclezole dose for chronic treatment. The half-life of Joreclezole was calculated as 7.5 days (1.2-14.0 days) and the clearance was found to be 38.8-302.6 $\mathrm{V} / \mathrm{day}$ (mean $99.1 \mathrm{l} / \mathrm{day}$ ). On the basis of these data the initial loreclezole dose was set at $40 \mathrm{mg} /$ day in 4 patients and $50 \mathrm{mg} /$ day in 9 patients. These data will be considered again in Chapter 8.

\begin{tabular}{|lllr|}
\hline $\mathrm{pt}$ & $\mathrm{T}_{\max }$ & $\mathrm{C}_{\max }$ & $\mathrm{T}_{1 / 2}$ \\
0701 & & & \\
0702 & 2 & 0.50 & 14.0 \\
0703 & 2 & 0.11 & 5.9 \\
0704 & 4 & 0.20 & 1.2 \\
0705 & 2 & 0.22 & 10.7 \\
0706 & 2 & 0.44 & 8.5 \\
0707 & 4 & 0.37 & 10.2 \\
0708 & 2 & 0.51 & 8.9 \\
0709 & 2 & 0.32 & 5.0 \\
0710 & 5 & 0.18 & 9.5 \\
0711 & 2 & 0.17 & 9.2 \\
0712 & 4 & 0.38 & 2.1 \\
0713 & 2 & 0.17 & 7.5 \\
& 4 & 0.31 & 4.9 \\
\hline
\end{tabular}

Table 3.3.a. Maximum plasma concentrations of loreclezole after a single oral dose of $50 \mathrm{mg}$. $T_{\text {max }}=$ time from ingestion to maximum plasma concentration in $h_{*} C_{m a x}=$ maximum plasma concentration in $m g / l_{1 / 2}=$ half life in days 
None of the patients reported adverse events and all tolerated the single dose of loreclezole well. No clinical, haematological or biochemical side effects were observed.

\subsection{Results of the chronic treatment study}

\subsubsection{Plasma concentrations}

On the basis of the data from the single dose study, 4 patients were treated with an initial loreclezole dose of $40 \mathrm{mg} / \mathrm{day}$ and 9 with $50 \mathrm{mg} / \mathrm{day}$ (Section 3.3.1). The dailly lorecllezole dose was about $0.78 \mathrm{mg} / \mathrm{kg} / \mathrm{day}$. When these doses had been given for about a month, the plasma concentrations were $0.45-1.97 \mathrm{mg} / \mathrm{J}$ (mean $\pm \mathrm{S} . \mathrm{E},=0.90 \pm 0.40$ $\mathrm{mg} / \mathrm{l})$, i.e. somewhat lower than the target plasma concentration of $1-2 \mathrm{mg} / \mathrm{l}$.

In 11 of the 13 patients the loreclezole dose was raised (to a daily dose of 50 to 100 $\mathrm{mg} /$ day) after about a month (second period). As a result the plasma concentration exceeded $1 \mathrm{mg} / \mathrm{in}$ all but one of the patients (range: $0.7-3.43 \mathrm{mg} / \mathrm{l}$, mean \pm S.E. $=1.70 \pm$ $0.80 \mathrm{mg} / \mathrm{l}$ ). Finally, after a further month (third period), the dose was raised $(70-120$

\begin{tabular}{llllllll|}
\hline $\mathrm{pt}$ & $\mathrm{I} / \mathrm{d}$ & $\mathbf{I} / \mathrm{I}$ & $\mathrm{II} / \mathrm{d}$ & III & III/d & III/I \\
& & & & & & \\
0701 & 0.64 & $\mathbf{0 . 6 9}$ & 0.64 & $\mathbf{1 . 1 7}$ & 0.77 & $\mathbf{2 . 0 2}$ \\
0702 & 0.69 & $\mathbf{0 . 6 6}$ & 0.97 & $\mathbf{1 . 9 6}$ & 0.97 & $\mathbf{2 . 0 2}$ \\
0703 & 0.63 & $\mathbf{0 . 4 5}$ & 1.25 & $\mathbf{0 . 7 1}$ & 1.50 & $\mathbf{1 . 1 6}$ \\
0704 & 0.74 & $\mathbf{1 . 3 5}$ & 0.74 & $\mathbf{2 . 7 0}$ & 0.74 & $\mathbf{3 . 4 0}$ \\
0705 & 0.83 & $\mathbf{0 . 7 8}$ & 1.00 & $\mathbf{1 . 3 1}$ & 1.17 & $\mathbf{1 . 9 7}$ \\
0706 & 0.51 & $\mathbf{1 . 0 3}$ & 0.63 & $\mathbf{1 . 8 0}$ & 0.63 & $\mathbf{1 . 8 9}$ \\
0707 & 0.82 & $\mathbf{1 . 2 8}$ & 1.02 & $\mathbf{2 . 5 0}$ & 1.02 & $\mathbf{2 . 5 0}$ \\
0708 & 0.63 & $\mathbf{0 . 5 3}$ & 0.89 & $\mathbf{1 . 0 3}$ & 1.27 & $\mathbf{2 . 8 4}$ \\
0709 & 0.65 & $\mathbf{0 . 7 7}$ & 0.65 & $\mathbf{1 . 1 1}$ & 0.81 & $\mathbf{1 . 5 1}$ \\
0710 & 0.60 & $\mathbf{0 . 7 4}$ & 0.72 & $\mathbf{1 . 7 9}$ & 0.84 & $\mathbf{2 . 1 7}$ \\
0711 & 0.60 & $\mathbf{1 . 9 7}$ & 0.60 & $\mathbf{3 . 4 4}$ & 0.60 & $\mathbf{3 . 6 4}$ \\
0712 & 0.68 & $\mathbf{0 . 7 5}$ & 0.82 & $\mathbf{1 . 1 8}$ & 0.96 & $\mathbf{1 . 2 4}$ \\
0713 & 0.71 & $\mathbf{0 . 9 9}$ & 0.89 & $\mathbf{1 . 3 4}$ & 0.89 & $\mathbf{1 . 5 6}$ \\
\hline
\end{tabular}

Table 3.4.a. Periods of the open add-on siudy. $I=$ first, $I I=$ second, $I I I=$ third period, $. . . / d=$ daily dose in $\mathrm{mg} / \mathrm{kg}$ body weight, .../ = plasma concentration in $\mathrm{mg} / \mathrm{l}$.

$\mathrm{mg} / \mathrm{day}$ ) in 5 patients (Table 3.4.a), so that the plasma concentration exceeded $1 \mathrm{mg} / \mathrm{lin}$ all the patients (range: 1.14-3.63 $\mathrm{mg} / \mathrm{l}$, mean $\pm \mathrm{SE} .=2.14 \pm 0.80 \mathrm{mg} / \mathrm{l})($ Fig. 3.4.b). In addition to measuring plasma concentrations and various parameters, pharmacokinetic calculations were performed. During chronic administration the half-life of loreclezola increased from, on average, 7.5 days after a single dose to 29 days (6.1-54.2 days), comparable to the value found in healthy wolunteers. The clearance fell from, on average, 99.1 to $35.3 \mathrm{~V}$ day. This justifies the administration of loreclezole once per day (Table. 3.4.c). 


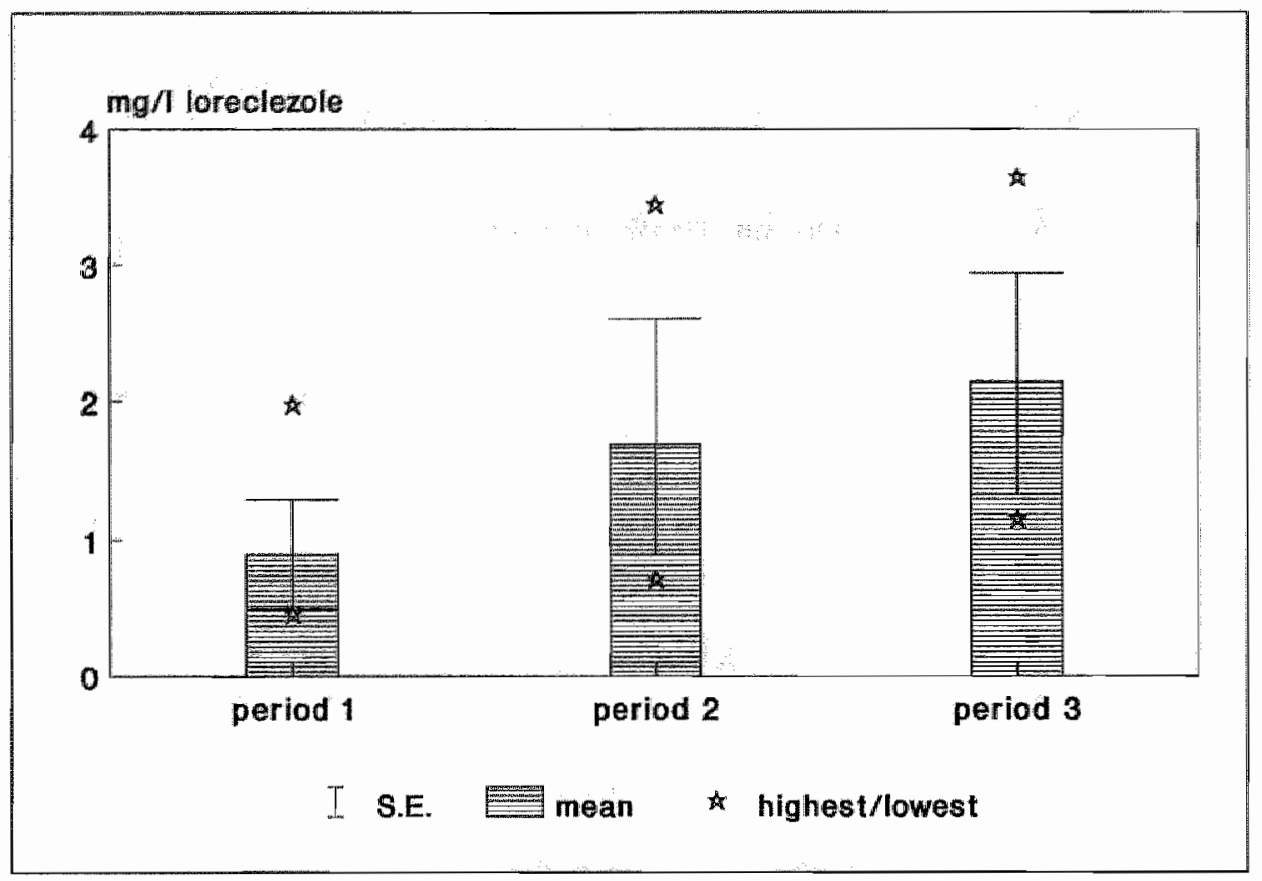

Fig. 3.4.6. Plasma concentrations of loreclezole, period 1 and $2: n=11$, period $3: n=10$

\begin{tabular}{|lrcc|}
\hline & Vd (I) & Clearance $(1 /$ day) & $T_{1 / 2}$ (days) \\
& & & 29.2 \\
mean: & 1322 & 35.3 & 16.3 \\
S.E.: & 930 & 17.4 & 6.1 \\
range & & & 54.2 \\
min.: & 340 & 9.0 & \\
\hline
\end{tabular}

Table 3.4.c. Kinerics in the open add-on study. $V d=$ volume of distribution, $T_{1 / 2}=$ half life in dons

To relate the effects of chronic loreclezole treatment on seizures to the existing medication, the seizure frequency was first calculated during a 3 -month baseline period. Comparison with the loreclezole treatment period was then possible. In the first period the mean seizure frequency fell from 0.61 seizures per day $(0.20-2.23 /$ day $)$ to $0.34 /$ day (0.06-2.32/day). After the loreclezole dose had been raised (to a daily dose of $50-100$ $\mathrm{mg} / \mathrm{day})$ in 11 of the 13 patients in the second period so that all but one of the patients had a plasma concentration greater than $\| \mathrm{mg} / 1$, the dose was again raised (70-120 mg/day) in 5 patients in the third period so that all patients then had a plasma concentration above $1 \mathrm{mg} / \mathrm{l}$ (mean $2.14 \mathrm{mg} / \mathrm{l}$ ). At the end of the study (after 6 months, in the third 
period), i.e. after raising of the loreclezole dose in 11 of the 13 patients, the metan daily dose was $0.94 \mathrm{mg} / \mathrm{kg}$ body weight (range $0.60-1.50 \mathrm{mg} / \mathrm{kg} /$ day) (Table $3.4 . a$ and Fig. 3.4.d). At 2, 4 and 6 months of treatment with loreclezole, the mean seizure frequency were 0.34 seizures/day (range $0.06-2.32$ ), 0.27 seizures/day (range $0.02-2.97$ ) and

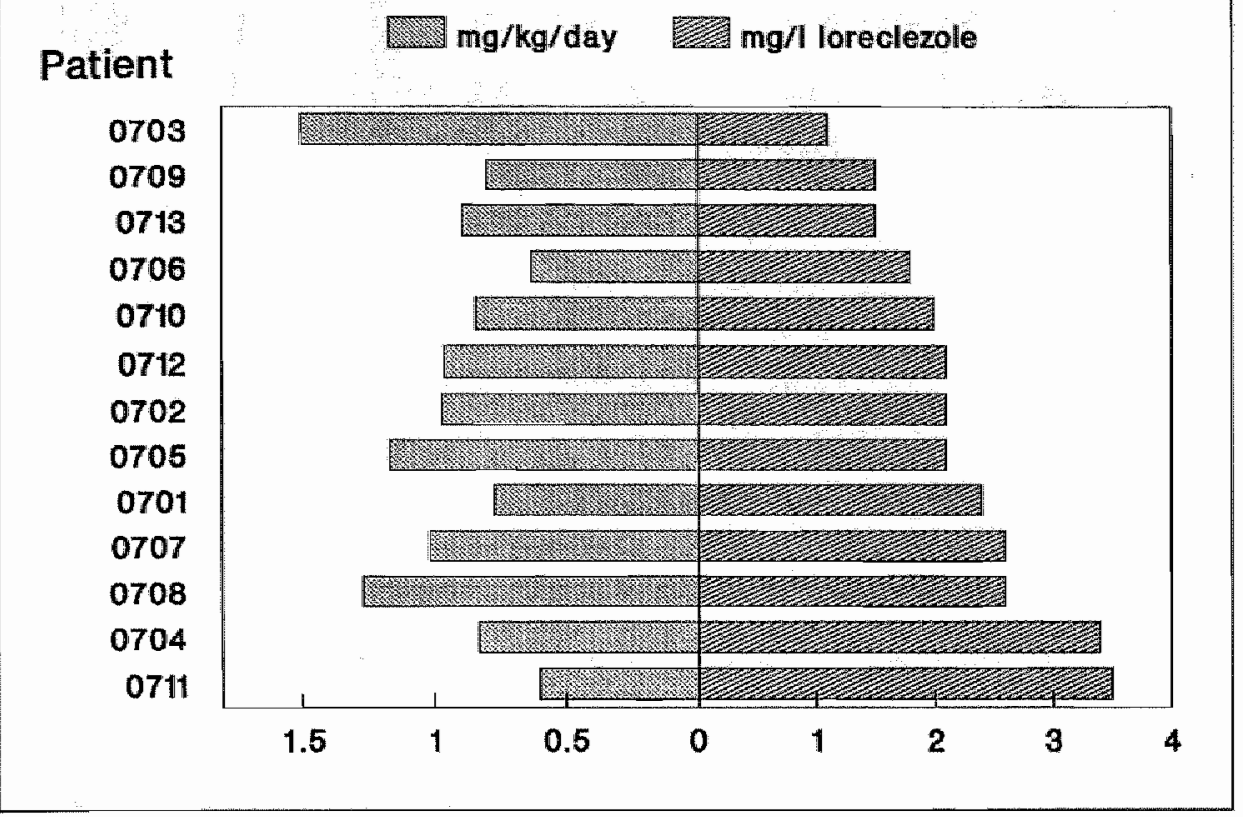

Fig. 3.4.d. Dose and plasma concentrations of loreclezole in period 3.

0.33 seizures/day (range 0.07-2.69). All 3 changes from baseline are signilicant (twotailed Wilcoxon test for intragroup comparisons: $p=0.001, p=0.003$ and $p=0.05$, respectively). During this study there was a mean seizure reduction of about $30 \%$; in 4 of the 13 patients the reduction in seizure frequency was at least $50 \%$ and remained unchanged in 9 patients (Fig. 3.4.e) Two patients reported a considerable shortening of the seizure duration: series of seizures lasting, on average, $2 \mathrm{~h}$ now lasted $10 \mathrm{~min}$ (patient 0705) and patient 0712 had seizures of shotter duration, albeit at a somewhat higher frequency. One patient (patient 0710) stopped using loreclezole owing to inadequate effect. Initially this patient experienced an increase of $32 \%$ in seizures, which however was within the usual range of fluctuation of frequency for this patient. This patient (patient 0710) and one other patient (patient 0707) decided to stop using loreclezole after the study because it produced no substantial reduction in the severity and ftequency of seizures.

The data for the patients who had used R 57720 (patients 0701, 0703, 0708, 0709 and 0713) are given in Table 3.4.f and Fig. 3.4.g. The antiepileptic efficacy of both substances seems evident. On the basis of this efficacy, 11 of the 13 participants wanted to continue using loreclezole after the end of the study. The results of the continued treatment. are discussed in Chapter 4. 


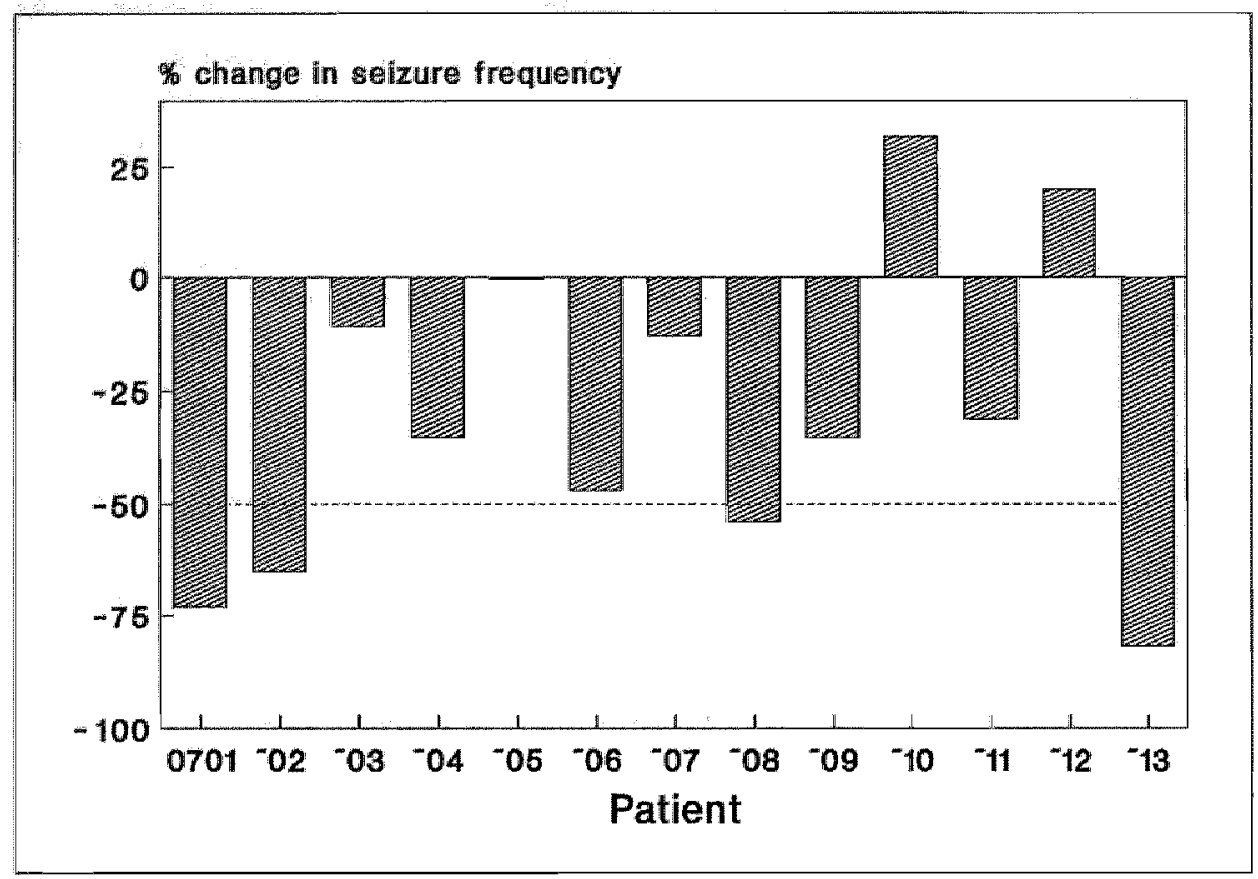

Fig. 3.4.e. Efficacy of loreclezole. Percentage change in seizure frequencyl 4 weeks compared to baseline period.

\begin{tabular}{|lccc|}
\hline pt & base & R 57720 & loreclezole \\
0701 & 23 & 1 & 7 \\
0703 & 49 & 13 & 41 \\
0708 & 16 & 2 & 10 \\
0709 & 13 & 8 & 9 \\
0713 & 17 & 13 & 4 \\
\hline
\end{tabular}

Table 3.4.f. Comparison of the resuls obtained with $R 57720$ and with loreclezole. base = baseline period, seizure frequency/4 weeks, $R 57720=$ mean seizure frequency/4 weeks during optimum (last period) treatment with $R 57720$, loreclezole = mean seizure frequency/4 weeks during treatmen with loreclezole (last period).

\subsubsection{Adverse events}

None of the patients reported adverse events and all tolerated the chronic loreclezole treatment well. There were no clinically rellevant side effects. One patient had headache, attributed to a plasma carbamazepine concentration that was somewhat too high for this patient. The symptoms disappeared after reduction of the carbamazepine dosage. One patient who had nausea and vomiting was found to be intolerant of capsules. This patient was considered as a drop-out. 


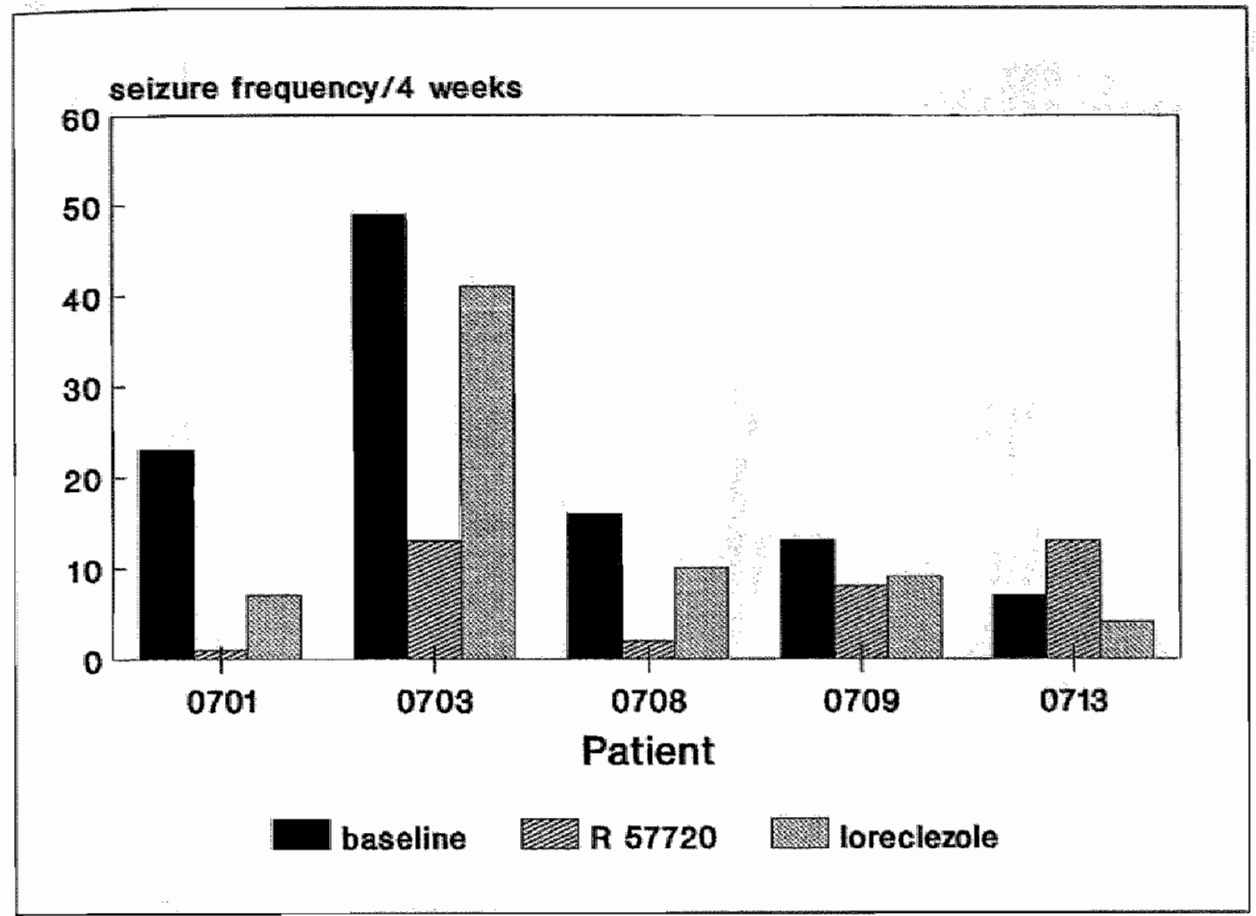

Fig. 3.4.g. Efficacy of $R 57720$ compared with loreclezole and baseline period.

During the study there was no effect on haematological or biochemical parameters, nor was there any notable influence on comedication apart from a slight increase in plasma phenytoin concentration (Figs. 3.4.h, 3.4.i, 3.4.j and 3.4.k). There were no signs of hepatotoxicity in this study. 


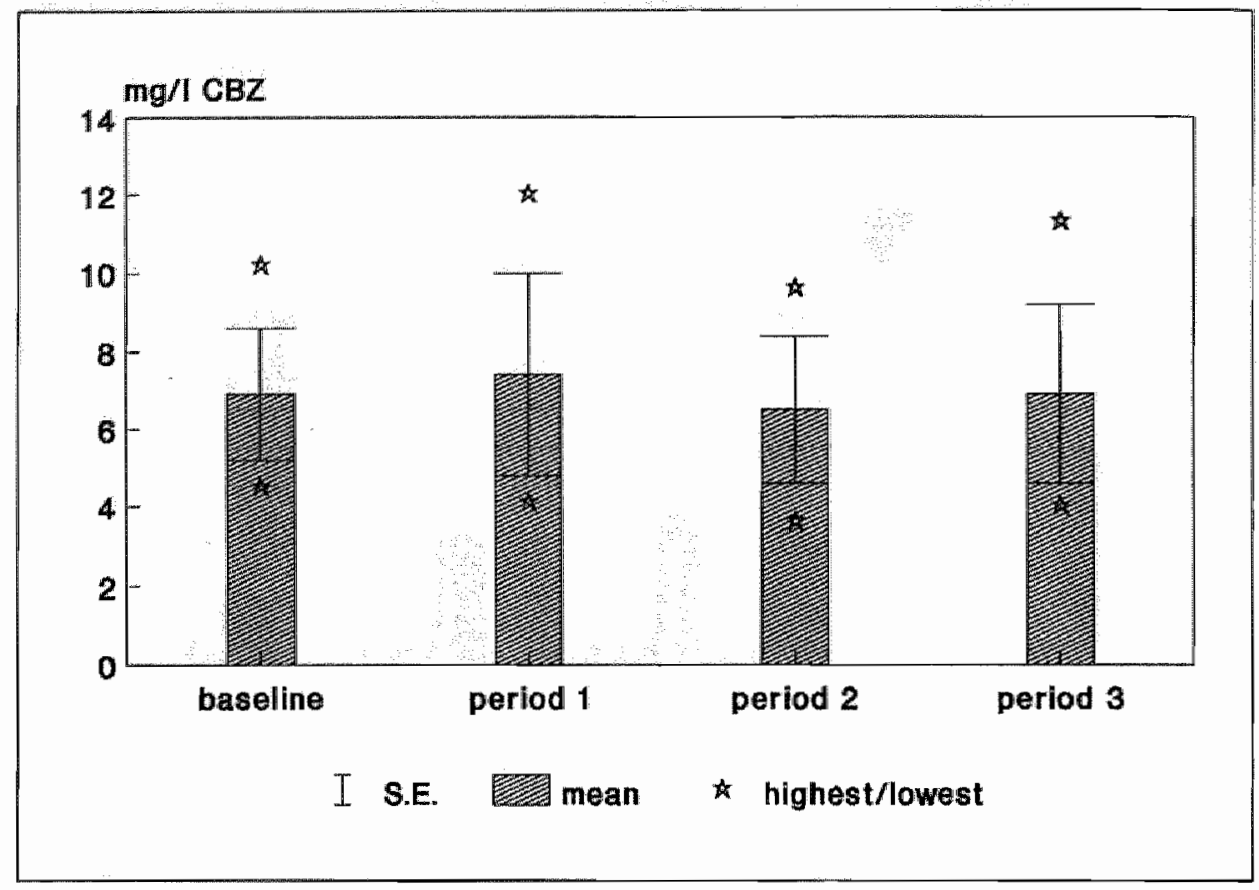

Fig. 3.4.h. Plasma concentrations of carbamazepine in treatment with loreclezole.

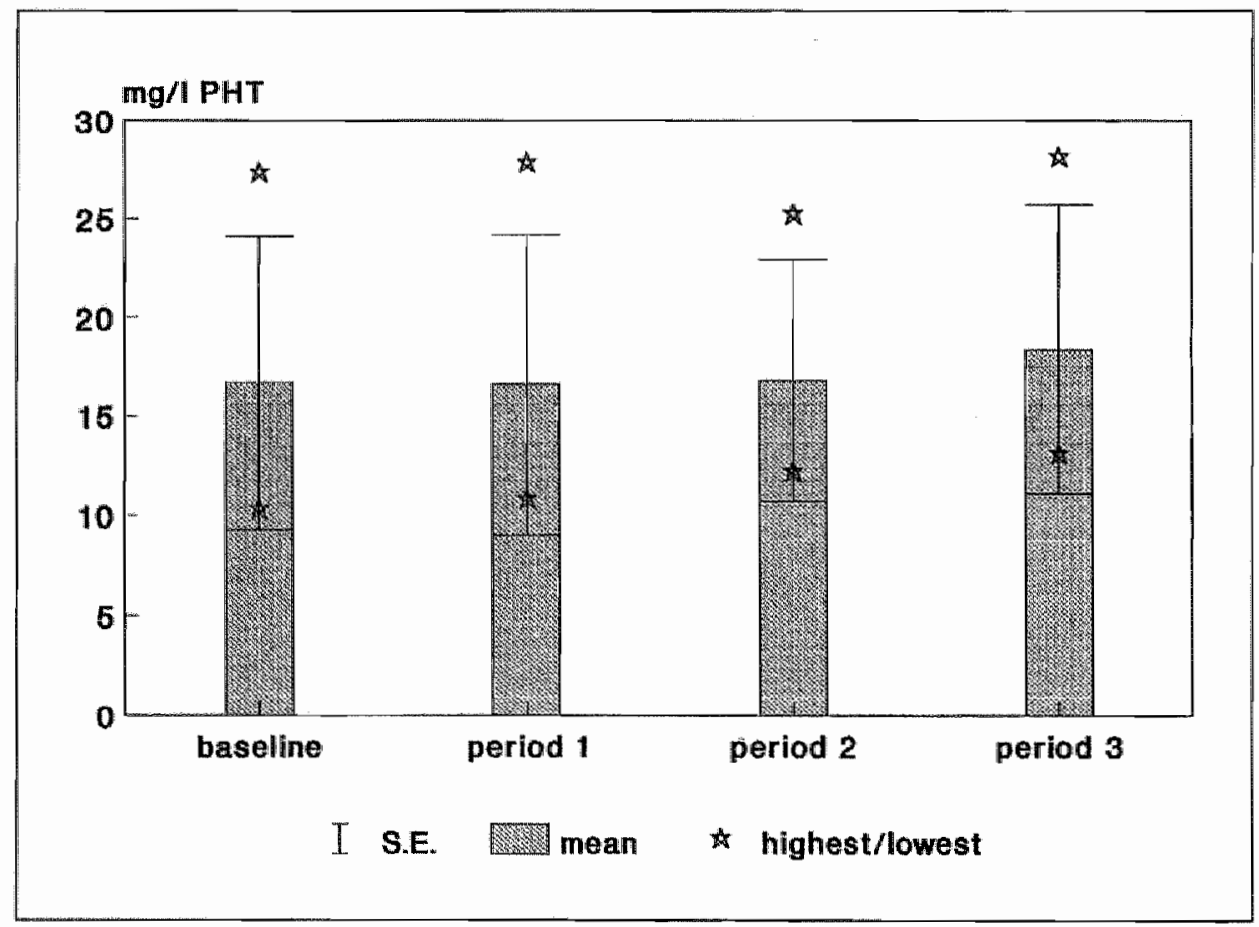

Fig. 3.4. i. Plasma concentrations of phenytoin in treatment with loreclezole. 


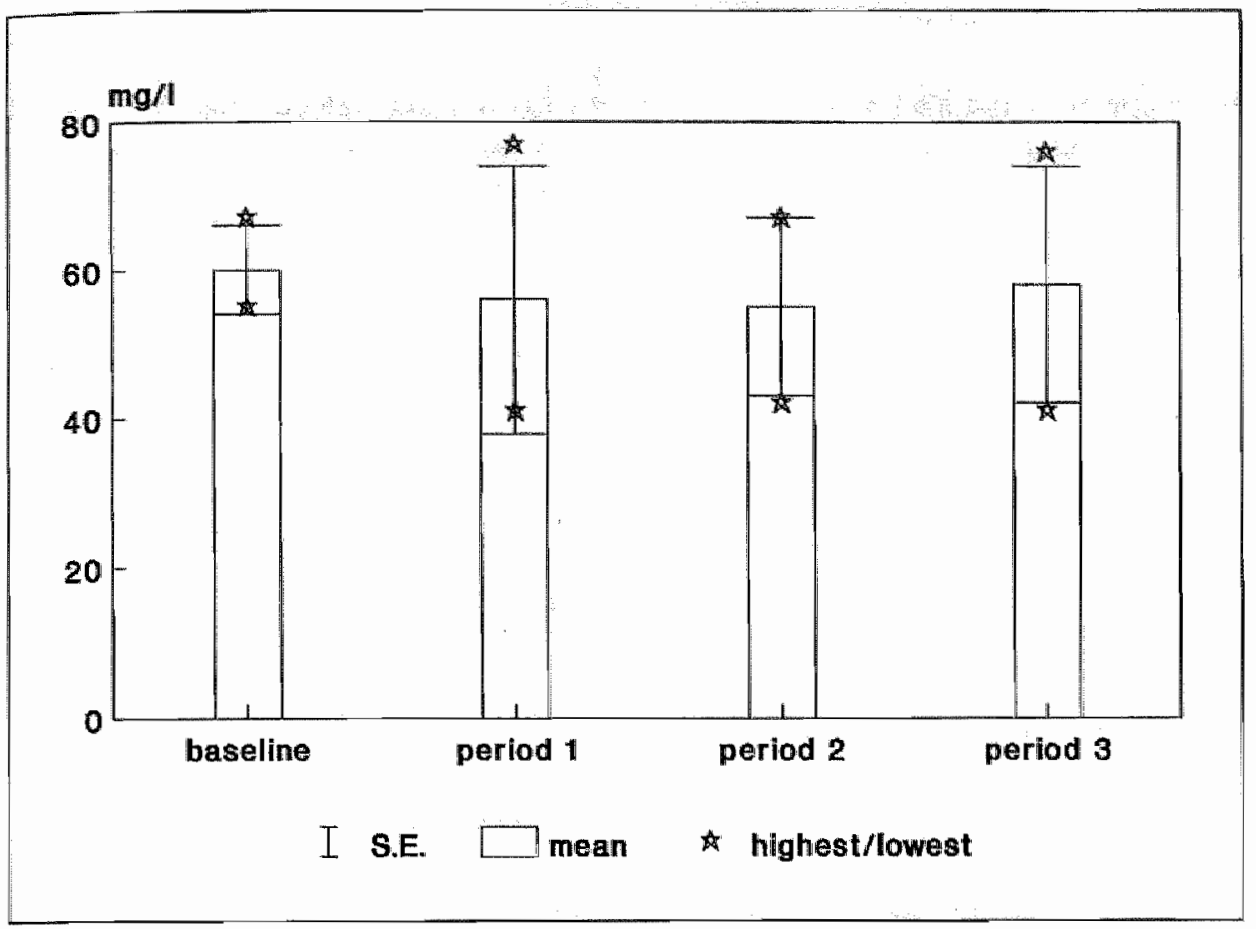

Fig. 3.4j. Plasma concentrations of valproate in treament with loreclezale.

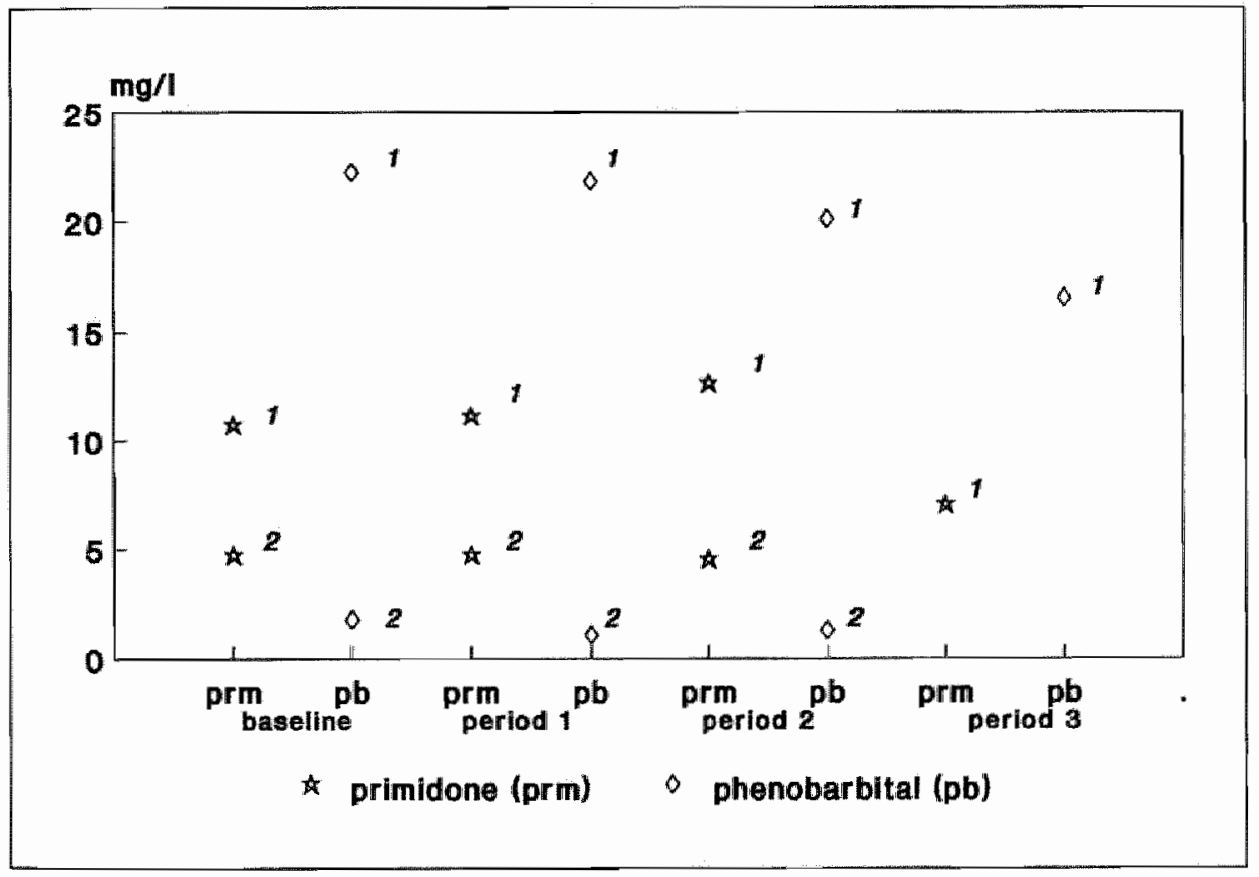

Fig. 3.4.k. Plasma concentrations of primidone in treatment with loreclezolebaseline, period I and $2: n=2$, period $3: n=1$ 
In this open study loreclezole was added to the existing antiepileptic medication of 13 pharmacotherapy-resistant epilepsy patients. The single dose and chronic administration of loreclezole were well tolerated by all patients and there was no influence on haematological or biochemical parameters. There was no notable influence on the plasma concentrations of the comedication (Fig. 3.5.a). The small differences that were observed in the mean plasma concentrations before and during loreclezole treatment did not displace the values used beyond the therapeutic range. For an individual patient, however, a small change in plasma concentration might indeed be noticeable and require a dose adjustment.

None of the patients reported side effects attributable to the use of loreclezole, nor were any such side effects detectable clinically. The dosage of $0.60-1.50 \mathrm{mg} / \mathrm{kg} / \mathrm{day}$, corresponding to daily doses of 50-120 mg given once per day, was tolerated well by all the patients.

It is possible that the low plasma concentrations found on administration of the single $50 \mathrm{mg}$ loreclezole dose for callculating the dose for chronic administration may have been caused by nonlinear kinetics, giving a discrepancy between the plasma concentrations reached at low doses and at high doses. This seems to differ from one individual to another (Section 8.2).

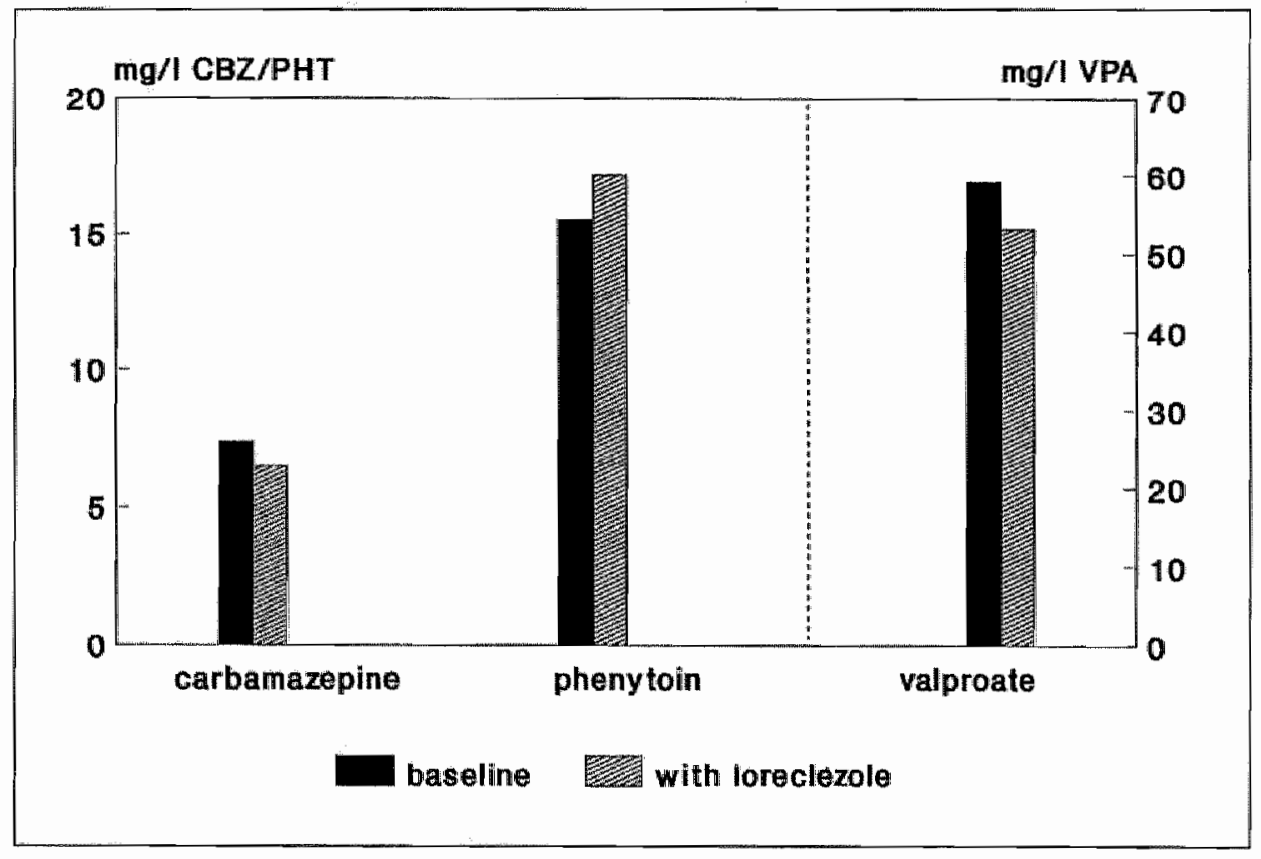

Fig. 3.5.a. Druginteractions: mean plasma concentrations in baseline and loreclezale treatment 
During chronic treatment the dose was gradually adjusted to such a level $(50-120$ $\mathrm{mg} / \mathrm{day})$ that the plasma concentration was between 1 and $3 \mathrm{mg} / \mathrm{l}$. The resulting mean dose of $0.9 \mathrm{mg}$ loreclezole $/ \mathrm{kg} /$ day $(0.60-1.50)$ was tolerated well by the 13 patients in this study. By raising the loreclezole dose the effect could be improved still further in 3 patients. In 1 patient there was no reduction in seizure frequency but the seizure duration was shortened. Although this is not reflected in an improved (reduced) seizure score, clinically it is a favourable result. In 2 patients the seizure frequency increased somewhat, but remained within the usual range for these patients. This increase in seizure frequency cannot be attributed to the use of loreclezole. In 1 of these patients the seizures were shifted from day to night, which the patient regarded as a favourable effect.

One patient had headache, attributed to an excessive plasma carbamazepine concentration. The symptoms disappeared when the carbamazepine dosage was reduced. One patient who reported nausea and vomiting was found to be intolerant of capsules.

\section{6}

\section{Conclusion}

In this open study in patients with serious epilepsy, loreclezole as add-on medication was tolerated well. In the first instance a single low dose $(50 \mathrm{mg})$ was given. After this single dose the half-life was relatively short (about 7.5 days) in comparison with the 28 days in volunteers in chronic treatment and about 25 days in patients continuing treatment with loreclezole. The explanation for this must lie in the difference in the rate and level at which the distribution volume is reached in induced patients. A single dose of $50 \mathrm{mg}$ loreclezole proved to be an unsuitable basis for calculating the dose for chronic treatment. The plasma concentrations that are found give no indication of the plasma concentration that will be produced by chronic administration of the same dose or a linearly calculated higher dose.

The patients in this study were suffering from pharmacotherapy-resistant epilepsy with partial seizures with complex symptomatology, sometimes with secondary generalization, or from symptomatic generalized epilepsy. In 10 of the 13 patients a reduction in seizure frequency was obtained at a plasma concentration of only about $1 \mathrm{mg} / 1$. The dosage did not differ substantially between the patients with an inadequate effect on seizures and the patients in whom the result was favourable. Neither the loreclezole dose in $\mathrm{mg} / \mathrm{kg} /$ day nor the plasma concentration differed, and so the difference in effect was not caused by a difference in dosage/treatment. It is possible, therefore, that at a higher dose and plasma concentration an improvement in seizure control might have been obtained. According to the protocol, however, a dose that would raise the plasma concentration above $3 \mathrm{mg} / \mathrm{l}$ was impermissible.

This study shows that, at the dosage used, loreclezole is a safe and effective antiepileptic drug in combination with existing antiepileptic drugs. On the basis of this study it was decided that a plasma loreclezole concentration of $1 \mathrm{mg} / \mathrm{l}$ represents the minimum effective value, whilst many patients can achieve further improvement by raising the dose. Owing to the long half-life of loreclezole an adequate dosage interval is necessary (1 dose per day in this study). Less frequent dosing that once per day is inadvisable in 
view of the increased risk of non-compliance.

From the findings of this study it was concluded that a single dose of $50 \mathrm{mg}$ is unsuitable as a basis for determining the dose for chronic treatment.

The study justified a double-blind placebo-controlled study to confirm the efficacy of loreclezole. 


\section{Chapter 4. EVALUATION OF ONE-YEAR TREATMENT WITH LORECLEZOLE;}

follow-up study after an open add-on study of the efficacy and safety of loreclezole

\section{1}

\section{Introduction}

The patients who took part in the roughly 4-month study into the safety and efficacy of loreclezole as add-on therapy generally benefited from the loreclezole therapy (Chapter 3). Approximately half of them experienced a reduction of $40 \%$ or more in the frequency of seizures, and the severity of the seizures was reduced too. The patients were therefore asked to continue with the loreclezole treatment. The aim of the present study was to check whether the benefit and safety persist and to investigate whether tolerance of loreclezole develops as it does with benzodiazepines (Section 1.4.6). The study objecti-

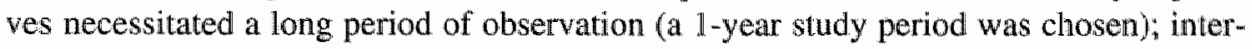
action with comedication could be assessed together with any biochemical or haematological effects or side effects and any clinical adverse reactions. In experimental medication the patients must be properly monitored so that any undesirable effects can be detected swiftly. That is one reason why the protocol for this follow-up study stipulated frequent observations.

\section{Methods and patients}

\section{Inclusion and exclusion criteria}

Patients who had taken part in the studies described in Chapter 3 were considered for the present study. Apart from the minimum seizure frequency, the inclusion and exclusion criteria were the same as in Section 3.2.1.

During the study the patient was to be withdrawn from the study in the event of:

- refusal of the patient to participate further (withdrawal of consent)

- clinically significant changes in thaematological and/or biochemical parameters

- any other serious disease or operation.

\subsubsection{Patients}

Patients were considered for this study if they had experienced a reduction of at least $40 \%$ in seizure frequency or an evident lessening of the severity of the seizures. This criterion was satisfied by 11 of the 13 patients. To exclude the possibility that any deterioration might be due to withdrawal of the conventional antiepileptic drugs, it was decided that in principle the comedication should be kept constant throughout the study year.

Loreclezole was given as a capsule $(10,25$ or $50 \mathrm{mg})$, in principle in the same dose with which the previous study, described in Section 3.4, closed. If it seemed likely that a higher dose would give a better result, the dose could be raised, the maximum permissible plasma concentration being $7 \mathrm{mg} / \mathrm{l}$. As mentioned above, the existing comedication (antiepileptic drugs) was maintained at the same dosage for the entire year unless there 
were important reasons for changing it, e.g. stressful or dangerous side effects possibly atributable to the comedication.

Investigations were performed monthly during the study period. These comprised an anamnesis (seizure frequency, seizure severity and adverse events), examination by the neurologist, thaematological and biochemical investigations, determination of plasma concentration of the antiepileptic drugs and determination of the plasma loreclezole concentration (Table 4.2.ai).

\section{$42.3 \quad$ Seizure severity score}

In an endeavour to obtain a simple method for weighting a change in the severity of seizures, an attempt was made to weigh the seizure on the basis of the patient's description. With the scoring system given below it was also possible to make a weighting retrospectively on the basis of the seizure diary if the patient had distinguished beween the various seizures. The system was so simple that the patient, the family or bystanders could also make use of it. The system comprised 5 scores:

- score $=1$ for absences and elementary partiall seizures,

- score $=2$ for complex partial seizures without incontinence and with a duration of less than 10 minutes (including post-ictal confusion),

- score $=3$ for all other complex partial seizures and for tonic-clonic seizures,

- score $=4$ for series of complex partial seizures and

- $\mathbf{s c o r e}=\mathbf{5}$ for status epilepticus.

For nocturnal seizures 1 point was subtracted from the weighting factor, with the exception of status epilepticus, for which the score remained 5 points.

\section{3}

\section{Results}

Of the 13 patients from studies described in Chapter 3,11 took part in the present study. These patients were of normal build (body mass index = Quetelet index: 19.1-26.1 $\mathrm{kg} / \mathrm{m} 2)$ and condition. During this study year none of the patients stopped the loreclezole treatment prematurely. Five patients required a change of comedication because

- plasma concentrations of loreclezole and other antiepileptic drugs, including metabolites and any free fractions, immediately after the first dose of the day

- haematological investigations: red and white blood cells, platelets, haemoglobin, haematocrit, sedimentation, differential blood count

- biochemical investigations: glucose, urea, creatinine, bilirubin, ASAT, ALAT, Y-GT, alkaline phosphatase, sodium, potassium, calcium, chloride, phosphate, total protein

Table 4.2.a. Laboratory determination. 
adverse events were suspected of being due to the comedication. These side effects were gastric symptoms attributed to acetazolamide in 3 patients (patients 0702, 0704, 0708; gradually withdrawn in all) and drowsiness in patients 0706 (attributed to primidone; which was slowly withdrawn), 0708 and 0712 (attributed to phenytoin in both cases; the daily dose was reduced by 25 and $50 \mathrm{mg}$ respectively). To cope with excessive stress caused by radical changes in his private life, patient 0701 took clorazepate as extra medication for 2 months. In 5 patients (patients 0703, 0709, 0711,0712 and 0713) the loreclezole dose was raised in the hope of obtaining better seizure control at a higher plasma concentration. The loreclezole dosage ranged from 50 to $150 \mathrm{mg} / \mathrm{day}$ with a

\begin{tabular}{|lllllrl|}
\hline pt & hgt & wgt & Q & $\mathrm{mg} / \mathrm{k}$ & $\mathrm{mg} / \mathrm{d}$ & $\mathrm{mg} / \mathrm{l}$ \\
0701 & 178 & 78 & 24.6 & 0.77 & 60 & 2.50 \\
0702 & 167 & 72 & 25.8 & 0.97 & 70 & 2.69 \\
0703 & 184 & 80 & 23.6 & 1.50 & 120 & 1.27 \\
0703 & 184 & 80 & 23.6 & 1.87 & 150 & 2.67 \\
0704 & 165 & 60 & 22.0 & 0.83 & 50 & 4.31 \\
0705 & 168 & 60 & 22.3 & 1.17 & 70 & 2.89 \\
0706 & 181 & 77 & 23.5 & 0.65 & 50 & 1.86 \\
0708 & 174 & 79 & 26.1 & 1.27 & 100 & 3.20 \\
0709 & 163 & 62 & 23.3 & 0.97 & 60 & 2.62 \\
0709 & 163 & 62 & 23.3 & 1.21 & 75 & 4.43 \\
0711 & 193 & 83 & 22.6 & 0.60 & 50 & 3.26 \\
0711 & 193 & 83 & 22.6 & 0.72 & 60 & 3.14 \\
0712 & 175 & 73 & 23.8 & 0.96 & 70 & 1.46 \\
0712 & 175 & 73 & 23.8 & 1.37 & 100 & 4.87 \\
0713 & 171 & 56 & 19.1 & 0.89 & 50 & 1.35 \\
0713 & 171 & 56 & 19.1 & 1.07 & 60 & 2.73 \\
& & & & & & \\
\hline
\end{tabular}

Table 4.3.a. Relationship between herght, weight and body bulld and dosage and plasma concentration. $Q=$ body mass index, (italics $=$ same patient after change of dose), $m g / k=$ dose in $\mathrm{mg} / \mathrm{kg} / \mathrm{day}, \mathrm{hgt}=\mathrm{he} \mathrm{ght}, \mathrm{mg} / \mathrm{d}=$ dose $\mathrm{in} \mathrm{mg} / \mathrm{day}, \mathrm{wg} t=$ weight, $\mathrm{mg} / \mathrm{l}=$ mean plasma concentration in $\mathrm{mg} / \mathrm{l}$

mean of $72.5 \mathrm{mg} /$ day. Referred to body weight, the dosage was $0.60-1.87 \mathrm{mg} / \mathrm{kg} / \mathrm{day}$ (mean $0.83 \mathrm{mg} / \mathrm{kg} / \mathrm{day}$ ) (Table 4.3.a).

\subsubsection{Plasmaconcentrations}

During the study the plasma loreclezole concentration ranged from 0.90 to $7.19 \mathrm{mg} / 1$, with a mean of $2.39 \mathrm{mg} / \mathrm{l}$. When the plasma concentrations were averaged intraindividually, the values ranged from 1.27 to $4.87 \mathrm{mg} / \mathrm{l}$. In patient 0703 an increase in loreclezole dosage from $120 \mathrm{mg} /$ day (= $1.5 \mathrm{mg} / \mathrm{kg} / \mathrm{day}$ ) to $150 \mathrm{mg} /$ day (= $1.9 \mathrm{mg} / \mathrm{kg} / \mathrm{day}$ ) produced a disproportionate increase from 1.27 to $2.67 \mathrm{mg} / \mathrm{l}$. On average the plasma concentrations of the comedication remained unchanged, although slight modification of the phenytoin dose was necessary in 2 patients (Section 4.3). 
Seizure frequency ranged from 0 per 4 weeks to 57 per 4 weeks. The mean seizure frequency per patient per 4-week period and change in seizure frequency are shown in Fig. 4.3.b and Table 4.3.c. The absolute seizure frequency per 4-week periods is shown in Table 4.3.d. The change in seizure frequency relative to the baseline period is given in Table $4.3 \mathrm{e}$, which also gives the corresponding values for the study described in Section 3.4. The table shows that 6 of the 11 patients had a reduction of $40 \%$ or more in seizure frequency, and the more stringent reduction of $50 \%$ was attained by 5 of the 11 . Relative to the baseline value, the mean number of seizures was $60.2 \%$ (range 35.1$97 \%)$, i.e. a mean improvement of about $40 \%(\mathrm{p}=0.001)$. Table 4.3.e shows that, relative to the study described in Section 3.4, the seizure frequency increased in patients 0701,0708,0709 and 0711, but fell further in patients 0702,0705 and 0706. On average, the change in seizure frequency during the year of the study represented an increase of $3 \%$ (range $-64.3 \%$ to $+53.4 \%$ ) relative to the study described in Section 3.4 $(p=0.83)$. In patients 0703 and 07.12 there was no clear reduction in mean frequency. but the seizures were of much shorter duration in patient 0703 and became shifted into the night in patient 0712 . On completion of this study year, all the patients continued to use loreclezole.

\subsubsection{Severity scoring}

For this studly the scoring system described in Section 4.2 .3 was monitored as thorough-

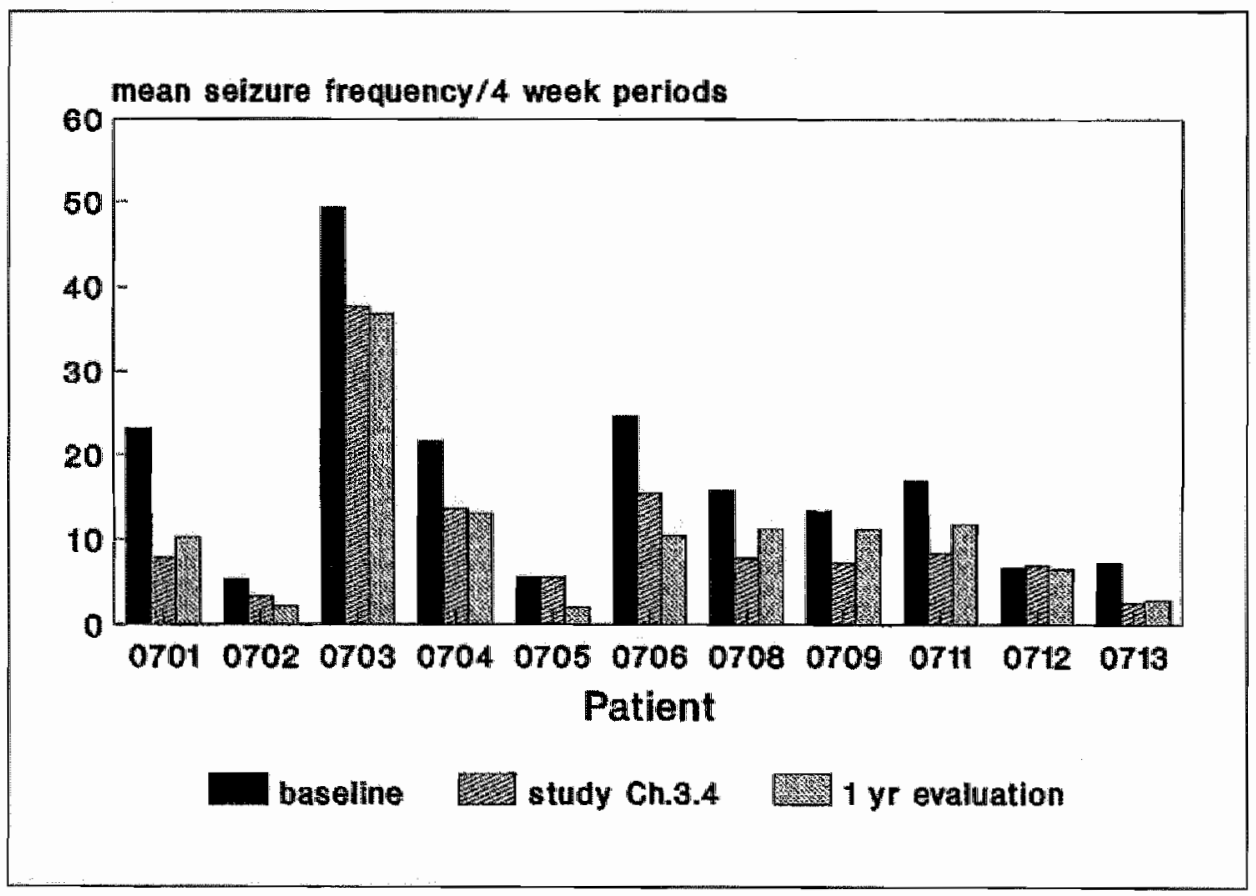

Fig. 4.3.b. Efficacy of lorectezole. Seizure frequency/4 weeks in baseline period compared with treatment with loreclezole (sindy described in Ch. 3.4) and 1 year follow-lip study. 


\begin{tabular}{|lrrr|}
\hline pt & sf 3 & sf 4 & \% ch \\
0701 & 7.9 & 10.2 & 129.1 \\
0702 & 3.3 & 2.2 & 66.7 \\
0703 & 37.7 & 36.8 & 97.6 \\
0704 & 13.6 & 13.1 & 96.3 \\
0705 & 5.6 & 2.0 & 35.7 \\
0706 & 15.4 & 10.4 & 67.5 \\
0708 & 7.9 & 11.3 & 143.0 \\
0709 & 7.3 & 11.2 & 153.4 \\
0711 & 8.5 & 11.8 & 138.8 \\
0712 & 7.0 & 6.5 & 92.9 \\
0713 & 2.6 & 2.9 & 111.6 \\
\hline
\end{tabular}

Table 4.3.c. Change in seizure frequency: seizure frequency in the study described in Section 3.4 versus the 1-year open add-on loreclezole study. sf $3=$ mean seizare frequency per 4 weeks in the study described in Section 3.4, sf $4=$ mean seizure frequency per 4 weeks in the study described in Chapter $4 \%$ ch = percentage change (sf 4 as a percentage of $s f 3)$.

\begin{tabular}{|c|c|c|c|c|c|c|c|c|c|c|c|c|c|}
\hline 0701 & 9 & 10 & 2 & 21 & 12 & 5 & 5 & 8 & 7 & 16 & 14 & 6 & 17 \\
\hline 0702 & 3 & 1 & 2 & 1 & 1 & 3 & $\mathbb{1}$ & 1 & 1 & 1 & 5 & 5 & 3 \\
\hline 0703 & 48 & 30 & 38 & 45 & 47 & 45 & 47 & 44 & 18 & 22 & 40 & 28 & 26 \\
\hline 0704 & 9 & 9 & 10 & 21 & 15 & 10 & 16 & 17 & 7 & 17 & 14 & 14 & 13 \\
\hline 0705 & 7 & 4 & 4 & 1 & 2 & 2 & 2 & 0 & 0 & 1 & 3 & 0 & 0 \\
\hline 0706 & 10 & 20 & 29 & 18 & 6 & 6 & 10 & 5 & 6 & 8 & 4 & - & . \\
\hline 0708 & 13 & 18 & 8 & 9 & 4 & 18 & 11 & 6 & 15 & 9 & 13 & 11 & 11 \\
\hline 0709 & 12 & 6 & 11 & 3 & 6 & 8 & 15 & 17 & 7 & 18 & 12 & 13 & 19 \\
\hline 0711 & 16 & 14 & 19 & 10 & 5 & 10 & 11 & 6 & 14 & 7 & 11 & 17 & 114 \\
\hline 0712 & 5 & 12 & 8 & 6 & 5 & 5 & 5 & 7 & 9 & 2 & 3 & 5 & 9 \\
\hline 0713 & 6 & 3 & 3 & 1 & 4 & 1 & 1 & 6 & 1 & 3 & 5 & 2 & 1 \\
\hline
\end{tabular}

Table 4.3.d. Number of seizures per 4-week periods.

ly as possible. It was found that the weighted score differed by roughly a factor of 2 from the score based on the frequency of seizures alone (Table 4.3.f and Table 4.3.g). If the score showed a clinically relevant improvement, however, this agreed with the improvement that was detectable in the frequency of seizures. Even the patients who reported a reduction in the severity of their seizure type showed no evident change in their weighted score unless the frequency of seizures had decreased too. It must be concluded that there is no detectable difference between the two methods of measurement. In this form the method seems useless for evaluating the effect. As it represented an additional burden for the patient it was not used in subsequent studies. The conventional seizure frequency measurement was retained as the criterion. 


\begin{tabular}{|lll|}
\hline pt & $3 / \mathrm{bl} \%$ & $4 / \mathrm{bl} \%$ \\
0701 & 34.1 & 44.0 \\
0702 & 62.3 & 41.5 \\
0703 & 76.5 & 74.6 \\
0704 & 62.7 & 60.4 \\
0705 & 98.2 & 35.1 \\
0706 & 62.3 & 42.1 \\
0708 & 50.3 & 72.0 \\
0709 & 54.4 & 84.2 \\
0711 & 50.0 & 69.4 \\
0712 & 104.5 & $97.0 *$ \\
0713 & 35.6 & 39.7 \\
\hline
\end{tabular}

Table 4.3.e. Efficacy of loreclezole: seizure frequency expressed as a percentage relative to the baseline period. $3 / \mathrm{bl}$ \% $=$ seizures in the study described in Section 3.4, as a percentage relative to baseline, $4 / \mathrm{bl} \%=$ seizures in the study described in Chapter 4 as a percentage relative to baseline. patien $07 / 2$ had milder seizures relative to baseline

\subsubsection{Adverse events}

There were no adverse events that could be attributed to the use of loreclezole. The addon loreclezole therapy was tolerated well by all patients. In 5 patients it became possible to reduce the comedication, which had led to adverse events in the form of drowsiness and gastric symptoms, without any detrimental effect on seizure frequency (Section 4.3).

\subsection{Discussion}

The 11 patients reported in Section 3.4 as having attained a satisfactory therapeutic outcome continued the loreclezole treatment. In 3 patients the loreclezole dosage remained unchanged. In 5 patients the comedication (phenytoin and acetazolamide) was reduced owing to adverse events, and in 1 patient the primidone medication was gradually withdrawn altogether. During the study year the seizure control remained stable at the level attained in the study described in Section 3.4. In 5 of the 11 patients the seizure frequency fell by more than $50 \%$ relative to the pretrial period (Fig. 4.4.a); the remainder showed a less favourable result in terms of frequency alone, but were satisfied with a less spectacular result or had shorter or less violent seizures.

Fig. 4.4.a shows the seizure frequency as a percentage of baseline value, whilst Fig. 4.4.b shows that during the study year the seizure frequency per 12-week period was the same as in the 12 weeks of the study described in Section 3.4. With the exception of just 1 patient the seizure control after 15 months' treatment with loreclezole was better than the pretrial value, i.e. there was no evidence of tolerance. Only in patient 0709 was there a trend towards an increase in seizure frequency relative to the baseline period; in the other participants in this study the seizure frequency in the last three 4-week periods 


\begin{tabular}{|c|c|c|c|c|c|c|}
\hline $\mathrm{pt}$ & $\begin{array}{l}\text { pretr } \\
\text { sf } / 4 w\end{array}$ & $\begin{array}{l}\text { pretr } \\
\text { se/4w }\end{array}$ & $\begin{array}{l}\text { oao } \\
\text { sf/4w }\end{array}$ & $\begin{array}{l}\text { oao } \\
\mathrm{sc} / 4 \mathrm{w}\end{array}$ & $\begin{array}{l}1 \mathrm{YT} \\
\mathrm{sf} / 4 \mathrm{~W}\end{array}$ & $\begin{array}{l}\text { lya } \\
\text { se/4w }\end{array}$ \\
\hline 0701 & 23.2 & $?$ & 7.9 & 18.0 & 10.2 & 21.1 \\
\hline 0702 & 5.3 & 16.3 & 3.3 & 5.5 & 2.2 & 4.4 \\
\hline 0703 & 49.3 & 90.7 & 37.7 & 68.4 & 36.8 & 60.6 \\
\hline 0704 & 21.7 & 44.7 & 13.6 & 30.8 & 13.1 & 22.1 \\
\hline 0705 & 5.7 & 11.3 & 5.6 & 10.3 & 2.0 & 4.0 \\
\hline 0706 & 24.7 & 73.3 & 15.4 & 44.9 & 10.4 & 22.4 \\
\hline 0707 & 29.0 & 56.0 & 25.0 & 46.9 & - & L. \\
\hline 0708 & 15.7 & 33.3 & 7.9 & 18.0 & 11.3 & 21.5 \\
\hline 0709 & 13.3 & 22.0 & 7.3 & 14.9 & 11.2 & 22,0 \\
\hline 0710 & 62.3 & 127.0 & 76.4 & 155.0 & - & 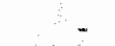 \\
\hline 0711 & 17.0 & 34.0 & 8.5 & 17.0 & 11.8 & 22.8 \\
\hline 0712 & 6.7 & 13.3 & 7.0 & 12.4 & 6.5 & 11.8 \\
\hline 0713 & 7.3 & 16.0 & 2.6 & 5.0 & 2.9 & 5.8 \\
\hline total & 258.0 & 537.9 & 210.3 & 423.6 & & \\
\hline total\# & 166.7 & 354.9 & 1089 & 221.7 & 108.2 & 197.4 \\
\hline
\end{tabular}

Table 4.3.f. Proposed scoring system for epileptic seizures, pretr $=$ pretrial, oao $=$ open add-on study with loreclezole (Chapter 3), lyr = one-year evaluation (Chapter 4), sfldw = seizure frequency per period of 4 weeks, sc $4 \mathrm{w}=$ score per period of 4 weeks, * = total score minus pt 0701, \# = total score minus pt 0701, 0707, and 0710. (for 0701 there were no exact seizure type data for the pretrial period; 0707 and 0710 stopped using loreclezole)

1. oao : pretr sf/4w (pt 070l excluded $)=0.815$

\section{2. oa0 : pretr score $/ 4 \mathrm{w}($ pt 0701 excluded $)=0.787$}

3. oao : pretr sf/4w (pt 0701, 0707, and 07/0 excluded) $=0.653$

\section{4. oa0 : pretr score $/ 4 \mathrm{w}$ (pt 0701,0707 , and 0710 excluded) $=0.625$}

5. 1yr : pretr sf $/ 4 \mathrm{w}(\mathrm{pt} 0701,0707$, and 0710 excluded $)=0.649$

\section{6. $1 \mathrm{yr}$ : pretr score $/ 4 \mathrm{w}$ (pt 0701,0707 , and 0710 excluded $)=0.556$}

Table 4.3.g. Ratios of scoring system; group comparisons. See Table 4.3.f.

was lower than in the baseline period.

The correlation between the prescribed daily loreclezole dosage and plasma concentration is not quite obvious: the patient with the lowest daily dosage $(50 \mathrm{mg} / \mathrm{day}=0.60$ $\mathrm{mg} / \mathrm{kg} /$ (day) had a mean plasma concentration of $3.26 \mathrm{mg} / 1$, whilst the patient with the highest dosage ( $150 \mathrm{mg} / \mathrm{day}=1.87 \mathrm{mg} / \mathrm{kg} / \mathrm{day})$ reached a plasma concentration of only 
\% selzure frequency/4 weeks period

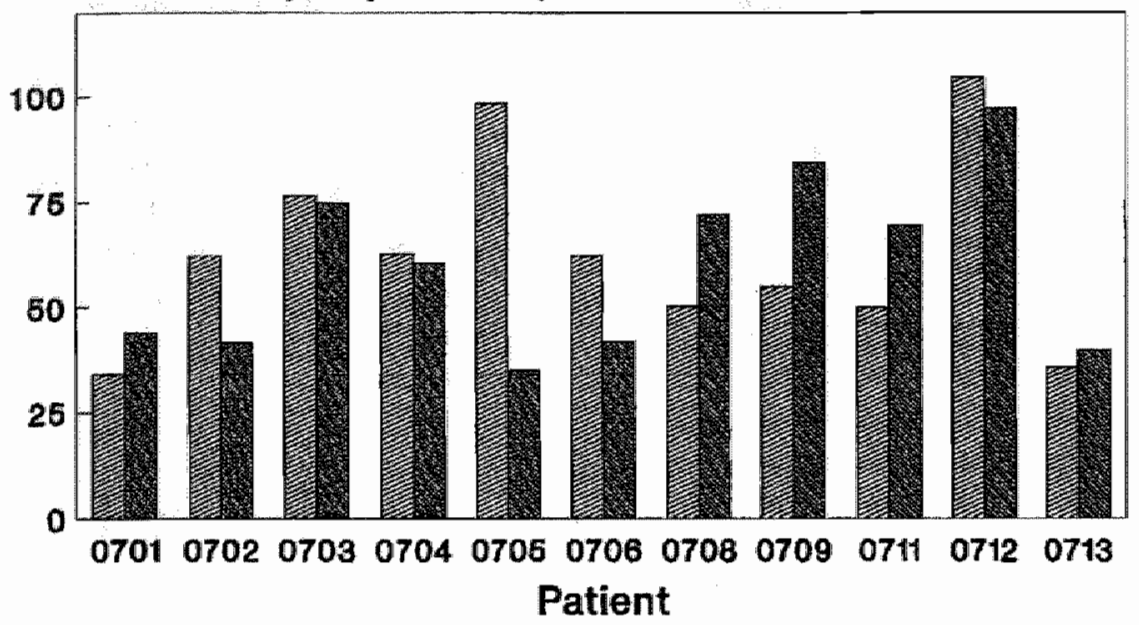

F of in 3.4/baseline of $1 \mathrm{yr} / \mathrm{baselin}$

Fig. 4.4.a. Efficacy of loreclezole. Seizure frequency/4 weeks, 12 weeks (Ch. 3.4) and l year trearment compared with baseline period.

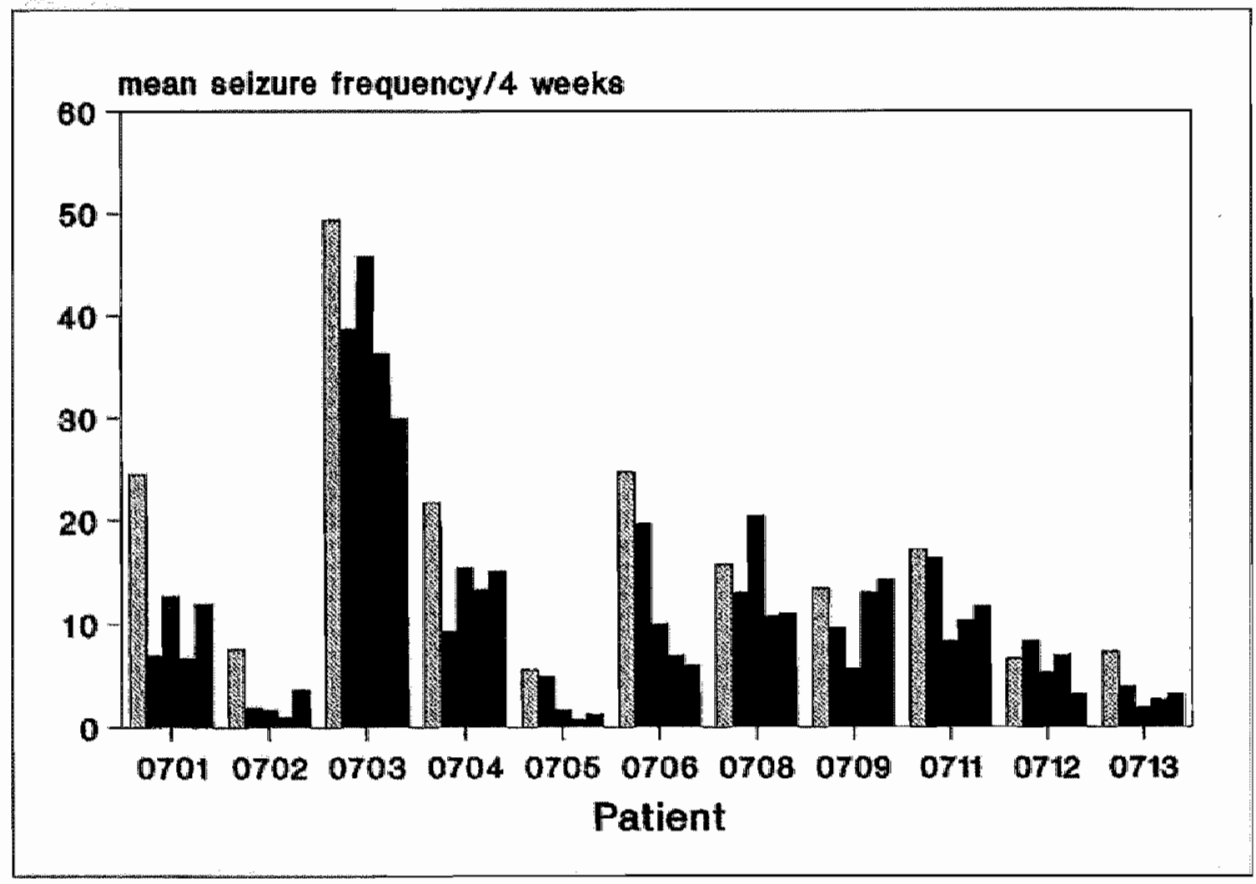

Fig. 4.4.b. Efficacy of loredezole. Seizare frequency/4 neeks in 1 year follow-up treatment compared with baseline period first barl. 
$2.67 \mathrm{mg} / \mathrm{l}$. There is no relationship between plasma loreclezole concentration and dose: The mean daily dose was $72.5 \mathrm{mg} / \mathrm{day}$, or $0.83 \mathrm{mg} / \mathrm{kg} / \mathrm{day}$; the mean plasma concentration was $2.4 \mathrm{mg} / \mathrm{l}$. An increase of $20 \%$ or $25 \%$ in the loreclezole dosage resulted in a doubling of the plasma concentration in patients 0703 and 0709 respectively, whereas a comparable dose increase in patient 0711 produced no detectable change in plasma concentration. This may be seen in Fig. 4.4.c, in which the plasma concentration per patient is shown in sequence of increasing dosage; for patients in whom the dose was changed the new dose is shown next to the original dose. As far as side effects are concerned, loreclezole distinguished itself well; there were no adverse events attributable to loreclezole.

After the end of the study described in Section 3.4, responders to loreclezole therapy were given the opportunity to continue with the same treatment. Eleven patients took part in this follow-up study. Some patients already had adequate seizure control at an initial dosage resulting in a plasma concentration of $1-2 \mathrm{mg} / \mathrm{l}$. Others were better treated at higher doses. During the year the loreclezole dose was raised in several patients and. the mean plasma concentration rose to $2.39 \mathrm{mg} / \mathrm{l}$. Thus the mean loreclezole dosage was $0.8 \mathrm{mg} / \mathrm{kg} / \mathrm{day}$, or $72.5 \mathrm{mg} / \mathrm{day}$. At this dosage the plasma concentration showed wide interindividual scatter, which is probably due to the wide interindividual variation in the metabolic clearance of this lipophilic substance. In addition, the clearance kinetics may be nonlinear, the degree of clearance depending on the actual plasma concentration. Loreclezole was tolerated well by all the patients in the study at the reported higher dosages. During this study year the seizure frequency was 10.8 seizures per 4 weeks as

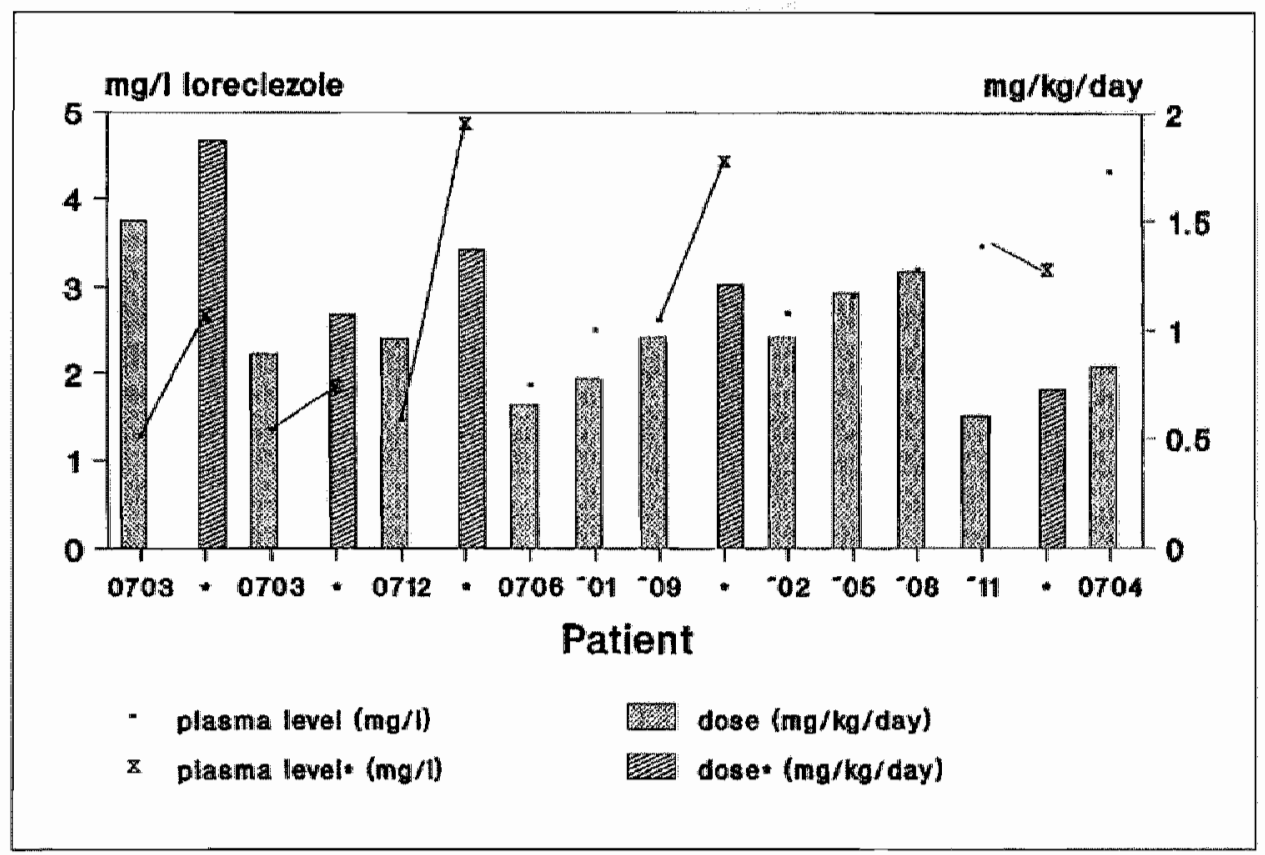

Fig. 4.4.c. Plasma concentrations (conc) and dose of loreclezole. asterix* indicates higher dose. 
apposed to 17.5 seizures per 4 weeks before the use of loreclezole and 10.6 seizures per 4 weeks in the study described in Section 3.4. There was no trend towards an increase in seizuire frequency during the year of the study. It may therefore be concluded that no tolerance of the therapeutic effeet of loreclezole developed during the 15 months of treatment. If should also be noted that several patients had shorter or less serious seizures. In some patients the comedication could be reduced somewhat without any impairment of seizure control. All patients wished to continue using loreclezole. This study too showed loreclezole to be a promising antiepileptic drug. 


\section{Chapter 5. DOUBLE-BLIND PLACEBO-CONTROLLED ADD-ON STUDY OF THE EFFICACY AND SAFETY OF LORECLEZOLE IN EPILEPSY PATIENTS*}

\section{1}

\section{Introduction}

From the open studies it was concluded that loreclezolle seems a promising antiepileptic drug, not only from animal studies but also from open add-on studies in pharmacotherapy-resistant patients (Chapter 3). To evaluate the efficacy of loreclezole objectively it was decided to perform a double-blind placebo-controlled, parallel study. In view of titration of loreclezole up to the desired plasma concentration an unblinded investigator was adopted to the study. To ensure an adequate number of patients a multicentre design was chosen. The aim of the study was to obtain a better understanding of:

1. the efficacy of loreclezole against epileptic seizures versus placebo, added to the existing antiepileptic medication,

2. the safety of loreclezole as add-on therapy, on the basis of clinical side effects, physical examination and laboratory findings,

3. the pharmacokinetic parameters of loreclezole after addition to the existing antiepileptic medication,

4. possible interactions between loreclezole and other antiepileptic drugs.

5.2

\section{Methods and patients}

\section{2 .1}

\section{Inclusion and exclusion criteria}

The inclusion criteria were comparable to those in the previous studies:

- well defined pharmacotherapy-resistant epilepsy

- at least 4 seizures of one type per month and no more than 14 conseculive days without a seizure

- plasma concentrations of antiepileptic drugs within the so-called therapeutic range, or such that they are well tolerated

- seizures that can be classified using the international classification

- participants must be capable of reporting adverse events carefully

- age 16 to 65 years

- good compliance.

The exclusion criteria were:

- seizure type with unclear clinical symptoms or such frequent seizures that accurate coumling is impossible

- mental retardation sufficient to prevent reliable reporting of adverse events

- progressive neurological diseases

\footnotetext{
"The results of this sndy are published as: Renmeester T. Janswen A, Hulsman I, Scholles" F, Van der Kleij B. Overweg J. Meijer J, De Bevkelaar F. A double-blind, placebo-controlled evaluation of the efficacy and safety of loreclezole as add-on therapy in patients with uncontrolled partiat seizurex. Epilepsy Res 1991:9:59-64
} 
- pregnancy or risk of pregnancy

- changes in the antieplleptic nedication in the month preceding the start of the trial

- history of serious physical disease (renal, hepatic, cardiovascular, gastrointestinal, haematological or malignant)

- drug or alcohol abuse

- participation in another study with experimental medication in the year preceding the present trial.

The participant was to be withdrawn from the study and regarded as a drop-out in the event of:

- a serious intercurrent disease

- serious adverse events

- influence on the comedication, causing a serious deterioration of the patient's condition

- pregnancy

- patient's decision to discontinue participation.

\subsubsection{Dosage}

From the results of the single administration of loreclezole and the subsequent open add-on chronic treatment of patients with loreclezole (Chapter 3), it was concluded that considerable differences in kinetics exist between a low single dose and chronic administration at higher doses in patients who are already taking antiepileptic drugs. A different dosage scheme was therefore chosen for the present study. The patients in the active substance group were treated with $100 \mathrm{mg}$ loreclezole once per day for 10 days. On the basis of the plasma concentrations measured on the 7th and the 14th day the dosages for the period from the $10 \mathrm{th}$ to the $17 \mathrm{th}$ day and for the subsequent period were determined in accordance with a scheme (Fig. 5.2.a), using the following criteria:

\section{if the plasma concentration on day 7 is:}

- greater than $0.75 \mathrm{mg} / \mathrm{l}$ : continue with $25 \mathrm{mg}$ loreclezole/day

- less than $0.75 \mathrm{mg} / \mathrm{l}$ : continue with $100 \mathrm{mg}$ loreclezole/day

\section{if the plasma concentration on day 14 is:}

- greater than $0.75 \mathrm{mg} / \mathrm{l}$ : continue with $25 \mathrm{mg}$ loreclezole/day

- less than $0.75 \mathrm{mg} / \mathrm{l}$ : continue with $50 \mathrm{mg}$ loreclezole/day.

The dosages were chosen to avoid loreclezole plasma concentrations over $2 \mathrm{mg} / \mathrm{l}$. The aim was a plasma loreclezole concentration between 1 and $2 \mathrm{mg} / \mathrm{l}$, taking the line that the daily dose of loreclezole had to be $25-50 \mathrm{mg}$. This target was chosen in view of the proven efficacy of loreclezole at this concentration in animal studies and the plasma concentrations of about $2 \mathrm{mg} / \mathrm{l}$ that proved to be effective and were well tolerated in a number of patients in the study described in Section 3.4.

Accurate registration of the seizure and side effect diary began 3 months before administration of placebo or loreclezole, to provide baseline values and accustom the patient to the study situation (baseline period). From 1 month before baseline period, so for a 


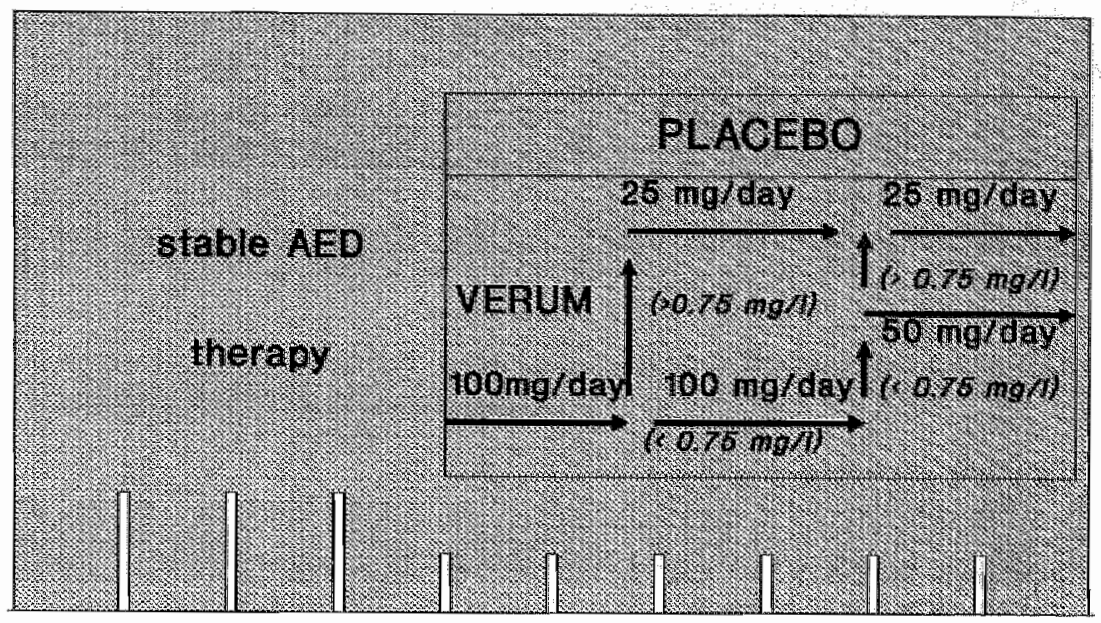

-12 wks-8 wks -4 wks day 0 day 7 day 10 day 14 day 17 ? day 17

Fig. 5.2.a. Study design of the double-blind, placebo-conrolled study. Days of evaluation are indicated.

period of 4 months before administration of loreclezole or placebo in all patients the medication was kept constant.

\subsubsection{Patients}

Sixty-six patients, some hospitalized, took part in the study. As 4 of them could not be evaluated the study results are based on 62 patients. The reasons for excluding the 4 patients were inaccurate recording of seizures ( 2 patients), insufficient motivation to follow the protocol ( 1 patient) and inadequate seizure frequency (1 patient). The evaluable patients comprised 19 women and 43 men. One patient was using no other antiepileptic drugs because all commercially available antiepileptic drugs had proved ineffective, 7 patients had monotherapy, 31 were taking 2 antiepileptic drugs, and the remaining 23 were taking 3 (Table 5.2.b). Fourty-one patients had epilepsy in the form of partial seizures with complex symptomatology, 19 had partial seizures with secondary generalization, and 2 had generalized epilepsy with various types of seizure (symptomatic generalized epilepsy). The patients were matched in pairs on the basis of their seizure frequency. The overall seizure frequency was the same for both groups. The patients were randomly given identical capsules containing placebo or $25 \mathrm{mg}$ active substance (loreclezole). After introduction of the new medication (active substance or placebo) the blood was tested every 14 days (Table 5.2.c) and, on the basis of the resulting plasma concentration, the loreclezole dosage was adjusted to a plasma concentration between 1 and 2 $\mathrm{mg} / 1$ and the placebo dosage was randomly adjusted by the unblinded investigator. The "blind" investigator examined the patients every 14 days in the first weeks and every 4 weeks thereafter, more frequently if necessary. In addition to the physical examination 


\begin{tabular}{|lll|}
\hline & $\begin{array}{l}\text { Ioreclezole } \\
(\mathrm{n}=32)\end{array}$ & $\begin{array}{l}\text { placelbo } \\
(\mathrm{n}=30)\end{array}$ \\
sex & $9 \mathrm{~F}, 23 \mathrm{M}$ & $10 \mathrm{~F}, 20 \mathrm{M}$ \\
age & $32(18-49) \mathrm{yrs}$ & $33(18-62) \mathrm{grs}$ \\
height & $177(160-208) \mathrm{cm}$ & $174(161-198) \mathrm{cm}$ \\
weilght & $71(47-97) \mathrm{kg}$ & $70(49-103) \mathrm{kg}$ \\
daily seiz. freq & $0.44(0.13-2.00)$ & $0.35(0.14-1.84)$ \\
number a.e.d.: & & \\
0 & & \\
1 & 1 & 0 \\
2 & 2 & 5 \\
3 & 19 & 12 \\
antiepileptic drugs: & 10 & 13 \\
CBZ/OXC & & \\
PHT & 25 & 28 \\
PB/PRM & 13 & 13 \\
VPA & 9 & 7 \\
CLB & 4 & 5 \\
other & 14 & 14 \\
& 3 & 3 \\
\hline
\end{tabular}

Table 5.2.b. Patient characteristics. $C B Z=$ carbamazepine, $P H T=$ phenytoin, $P R M=$ primidone, $C L B=$ clobazam, $O X C=$ oxcarbazepine, $P B=$ phenobarbital, $V P A=$ walproate.

- plasma concentrations of loreclezole and other antiepileptic drugs, including metabolites and any free fractions

- haematological investigations: red and white blood cells, platelets, haemoglobin, haematocrit, differential blood count

- bilochemical investigations: glucose, urea, creatinine, uric acid, cholesterol, bilirubin, ASAT, ALAT, $\gamma$-GT, LDH, alkaline phosplatase, folic acid, sodium, potassium, calcium, chloride, phosphate, total protein, albumin

Table 5.2.c. Laboratory determination.

the seizure diary was assessed and adverse events were noted.

After 3 months the patient and investigator were informed of the identity of the medication, but not until both the patient and the investigator had given their evaluation of the efficacy and tolerability of the treatment. Before the code was broken the investigator and the patient were asked to give an opinion of the treatment: was it active substance or placebo, and on what is your judgement based? All the patients were then given the 
opportunity to continue loreclezole treatment or - if they had been receiving placebo - to start it (Chapter 6).

\subsubsection{Statistical analysis}

In this study the numbers of responders ( $\geq 50 \%$ decrease in seizure frequency) in the two treatment groups (loreclezole vs placebo) were compared using Fisher ${ }^{*}$ exact probability test. For within-group comparisons of the changes in seizure frequency versus baseline, a Wilcoxon matched-pairs signed-ranks (mpsr) test was applied. For intergroup comparisons (loreclezole ws placebo) of the changes in seizure frequency. a Mann-Whitney U-test was applied (Siegel and Castellan 1988). A p-value $\leq 0.05$ (twotailed) was considered statistically significant.

\section{3}

\section{Results}

The active substance group and placebo group comprised 32 and 30 patients respectively. The patient characteristics are given in Table 5.2.b. In addition to sex, age, etc. the table gives data on the antiepileptic drugs used. The two groups were of comparable composition.

\subsubsection{Plasma concentrations}

On the basis of the plasma loreclezole concentration measured on day 7 the unblinded investigator decided whether the loreclezole dose to be giwen from day 10 should be reduced to $25 \mathrm{mg} / \mathrm{day}$ (for plasma concentrations exceeding $0.75 \mathrm{mg} / \mathrm{l}$ ) or should be kept at $100 \mathrm{mg} / \mathrm{day}$ (for concentrations less than $0.75 \mathrm{mg} / \mathrm{l}$ ). Similarly, on the basis of the plasma concentrations measured on day 14 , the dose to be given from day 17 was set at $25 \mathrm{mg} /$ day for 9 patients and at $50 \mathrm{mg} /$ day for 13 patients. In addition, 2 patients were prescribed $12.5 \mathrm{mg} /$ day and 8 patients $75 \mathrm{mg} /$ day and 3 patients $100 \mathrm{mg} /$ day on the basis of loreclezole plasma concentrations, in order to reach the preset target plasma concentration of $1-2 \mathrm{mg} / \mathrm{l}$.

By performing monthly plasma concentration measurements an attempt was made to keep the plasma loreclezole concentration between $\|$ and $2 \mathrm{mg} / \mathrm{l}$. To achieve this an adjustment of the loreclezole dosage (from 12.5 to a maximum of $125 \mathrm{mg} / \mathrm{day}$ ) was necessary in 24 patients. At the end of the study the most commonly prescribed dosage was $75 \mathrm{mg} / \mathrm{day}$ and the mean dosage was $67 \mathrm{mg} /$ day. The mean plasma loreclezole concentration was $1.70 \mathrm{mg} /$ (range $1.05-2.69 \mathrm{mg} / \mathrm{l}$ ). The protocol excluded a change of comedication dosage in the absence of any compelling reason such as serious intaraction. Averaging over the whole group, there was no change in plasma carbamazepine concentration or in dihydroxy - carbamazepine/carbamazepine ratio or carbamazepine10,11-epoxide/carbamazepine ratio or in mean phenytoin concentration. At an individual level $\mathbb{1}$ patient required a reduction of carbamazepine dosage and 2 patients required a reduction of phenytoin dosage on account of adverse events attributed to carbamazepine or phenytoin respectively. Despite a wide scatter in plasma clobazam concentrations there was evident interaction of loreclezole with the desmethylclobazam/clobazam ratio, which was significantly lower in the active substance group than in the placebo group 


\begin{tabular}{|lllll|}
\hline AED & P/V & $n$ & base & end \\
CBZ & & & & \\
& $\mathrm{V}$ & 28 & $7.35 \pm 0.35$ & $6.54 \pm 0.35$ \\
& $\mathrm{P}$ & 24 & $6.88 \pm 0.32$ & $6.54 \pm 0.35$ \\
$\mathrm{epx} / \mathrm{CBZ}$ & $\mathrm{V}$ & 23 & $0.19 \pm 0.01$ & $0.22 \pm 0.02$ \\
& $\mathrm{P}$ & 21 & $0.22 \pm 0.02$ & $0.24 \pm 0.03$ \\
diol/CBZ & $\mathrm{V}$ & 23 & $0.41 \pm 0.04$ & $0.47 \pm 0.04$ \\
$\mathrm{PHT}$ & $\mathrm{P}$ & 21 & $0.43 \pm 0.04$ & $0.43 \pm 0.04$ \\
& $\mathrm{~V}$ & 13 & $14.8 \pm 1.11$ & $16.1 \pm 1.22$ \\
& $\mathrm{P}$ & 14 & $12.7 \pm 0.80$ & $13.6 \pm 0.87$ \\
$\mathrm{CLB}$ & $\mathrm{V}$ & 13 & $144.5 \pm 26.8$ & $31.4 .9 \pm 43.5$ \\
& $\mathrm{P}$ & 14 & $131.4 \pm 25.2$ & $154.4 \pm 28.2$ \\
$\mathrm{DMC} / \mathrm{CLB}$ & $\mathrm{V}$ & 13 & $15.3 \pm 2.5$ & $7.8 \pm 0.9$ \\
& $\mathrm{P}$ & 14 & $16.6 \pm 3.0$ & $15.0 \pm 2.8$ \\
& & & & \\
\hline
\end{tabular}

Table 5.3.a. Plasma anitepileptic drug concentrations and metabolic ratios before and at the end of the trial (mean \pm S.E.). CBZ = carbamazepine $(m g / l), c p x=C B Z-10,11$-epoxide, diol $=$ dihydroxy $C B Z, P H T=$ phenytoin $(m g / l), C L B=\operatorname{clobazam}(n g / l), C L B=\operatorname{clobazam}(n g / l), D M C=$ deswrethylclobazam, $P=$ placebo group, $P=$ placebo group, $V=$ loreclezole group, $n=$ number, base $=$ baseline walue, end $=$ nalwe at the end of the trial.

(p<0.0001) (Table 5.3.a). Adjustment of the clobazam dosage was unnecessary, however, as the relevance of this observation is not clear (Schmidt et al. 1986). Regarding valproate plasma concentrations no changes were observed. However, the number of patients using valproate was too small to permit statistical evaluation with regard to interaction.

After 3 months of treatment the mean plasma loreclezole concentration in the active substance group was $1.69 \mathrm{mg} / \mathrm{l}(1.05-2.69 \mathrm{mg} / \mathrm{l})$ and the mean loreclezole dosage was $67 \mathrm{mg} / \mathrm{day}(25-125 \mathrm{mg} / \mathrm{day})$.

\subsubsection{Efficacy}

On screening for age, weight, height, seizure frequency and number of antiepileptic drugs, no differences were found between the two groups (Mann-Whitney U-test, $\mathrm{p}<0.05$, two tailed). In the pretrial phase the seizure frequency was somewhat higher in the active sulbstance group than in the placebo group but the difference was not significant: $0.44(0.13-2.00)$ seizures per day in the active substance group and $0.35(0.14-$ 1.84) seizures per day in the placebo group. Both in the active substance group and in the placebo group there were changes in seizure frequency in the course of the 3-month study period. In the loreclezole group the median seizure frequency fell from 0.44 to 0.36 (scatter $0.07-2.63$ ) seizures per day $(\mathrm{p}=\mathrm{N}$.S. Wilcoxon matched-pairs, signed rank test) (Fig. 5.3.b). In the placebo group there was a nonsignificant increase from 0.35 to 0.38 (scatter $0.13-2.11$ ) seizures per day $(\mathrm{p}=\mathrm{N} . \mathrm{S}$ ) (Fig. 5.3.c). In the placebo group the 


\section{$\%$ change in seizure frequency}

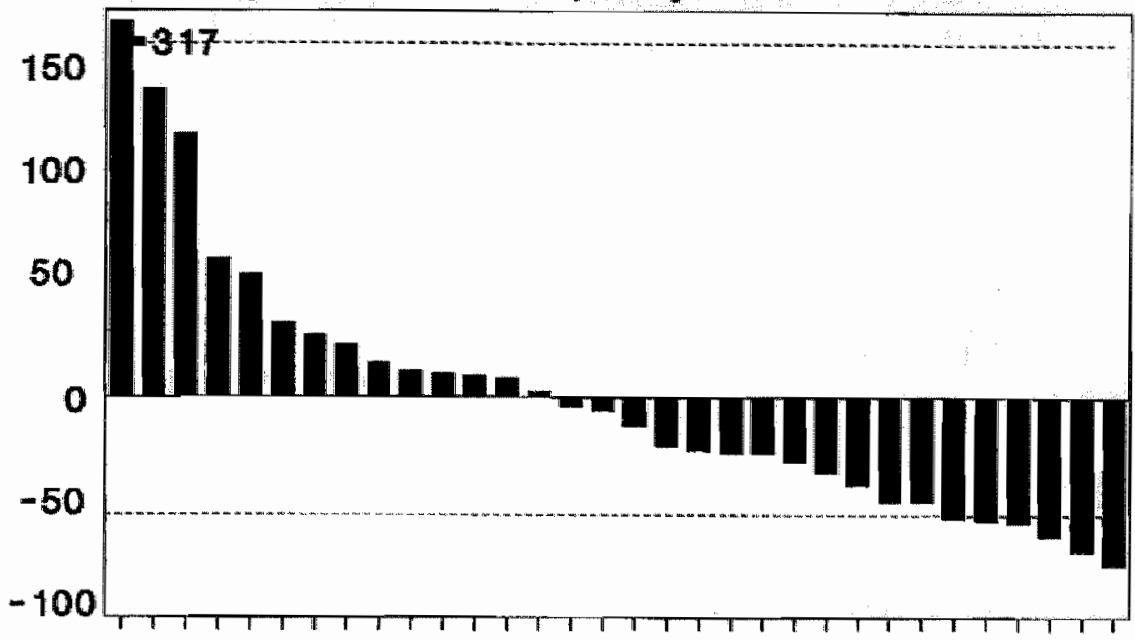

patients

Fig. 5.3.6. Efficacy of loreclezole. Seizure frequency/4 weeks in patients treated with verum.

greatest reduction in seizure frequency was $42 \%$, whilst in the loreclezole group 8 patients achieved a better result than this $42 \%$ (Fisher's exact test, $8 / 32$ versus $0 / 30, p=$ 0.005 ). In the loreclezole group 6 patients attained a reduction of more than $50 \%$ in seizure frequency, in the placebo group nobody did $(\mathrm{p}=0.002)$ (Fig. 5.3.d).

Plasma loreclezole concentrations did not differ substantially between patients with insufficient reduction in seizure frequency and patients with a seizure frequency reduction of $50 \%$ or more Iplasma concentration (mean \pm S.E.) $1.73 \pm 0.38 \mathrm{mg} / \mathrm{in}$ responders and $1.60 \pm 0.43 \mathrm{mg} / \mathrm{l}$ in nonresponders] (Fig. 5.3.e). There was no difference in seizure type either. This analysis takes no account of the fact that some patients reported a reduction in the severity of their seizures during loreclezole treatment and that the seizures no longer generalized. This improvement, which had allready been seen in the study described in Section 3.4.2, is not apparent simply from the seizure frequency diaries.

\subsubsection{Adverse events}

As stated in Section 5.3.1, there was no significant influence on mean plasmat carbamazepine or phenytoin concentrations. Although there was thus no significant decrease or increase in the mean plasma concentrations of these substances (or of carbanazepine10,11-epoxide or dihydroxycarbamazepine), there were 3 patients in whom the comedication had to be reduced on account of adverse events (ataxia, diplopia and muscle stiffness) which, on the basis of the plasma concentrations, was attributed to the medication. The symptoms disappeared in all 3 patients after this dose reduction. They all 
\% change in selzure frequency

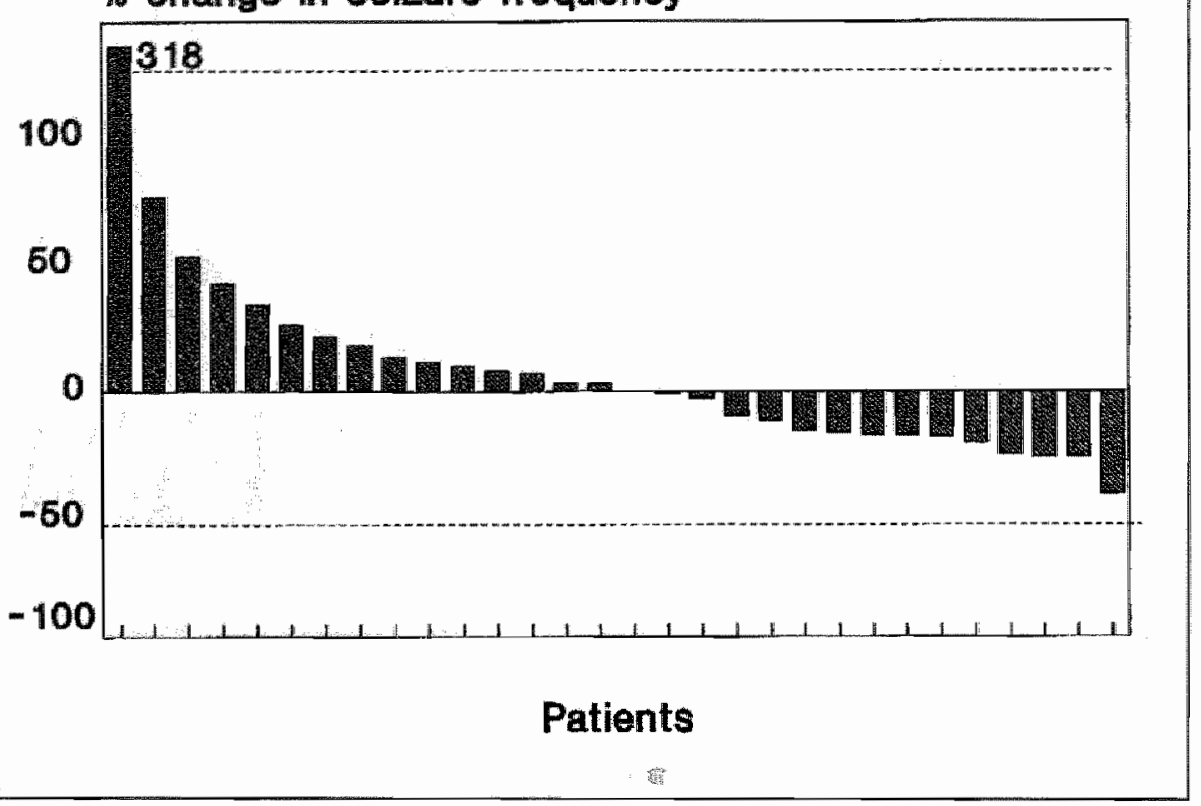

Fig. 5.3.c. Efficacy of loreclezole. Seizure frequency/4 weeks in patients treated with placebo.

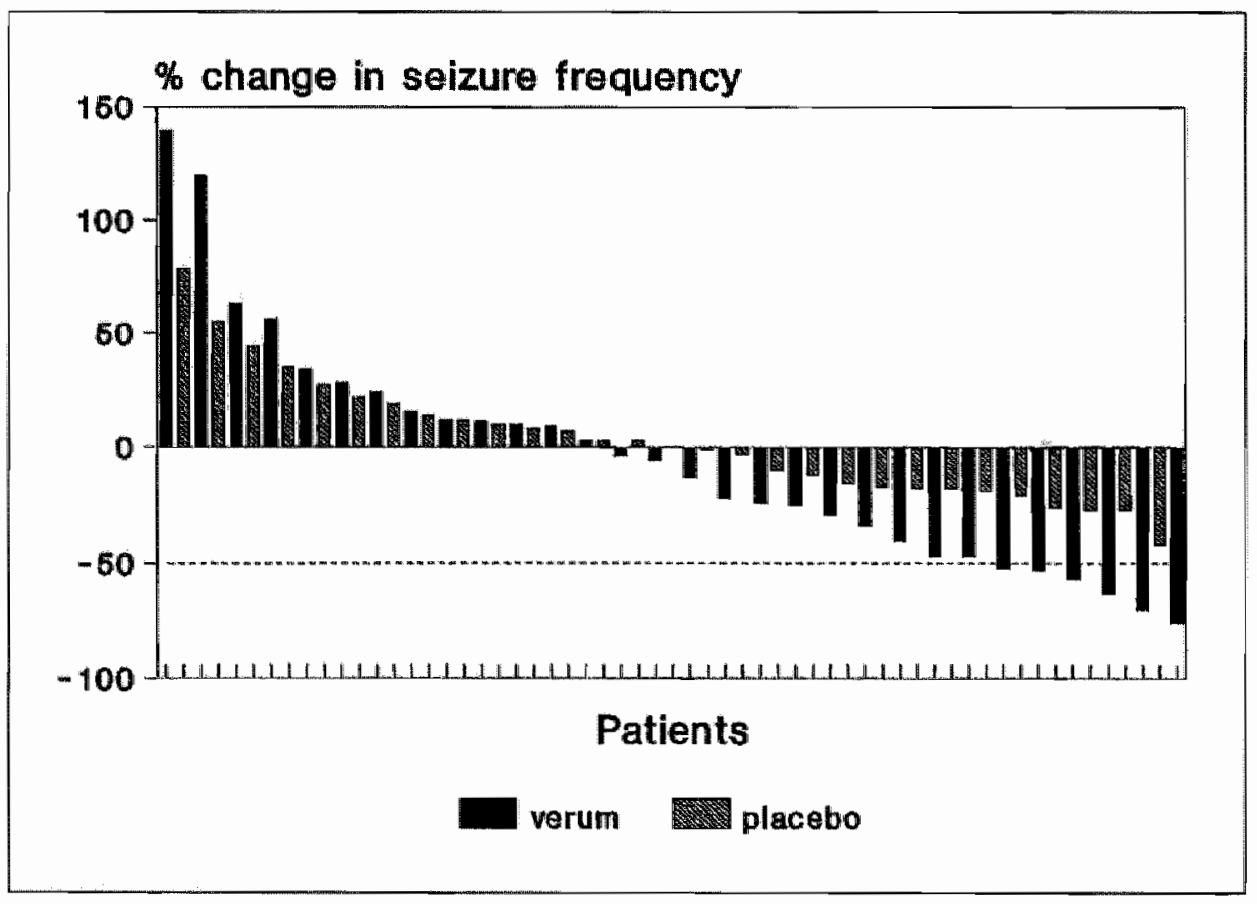

Fig. 5.3.d Efficacy of loreclezole. Percentage change in seizure frequency/4 weeks compared to boseline periond. 


\section{Patlents}

\section{change in sf mg/l loreclezole}

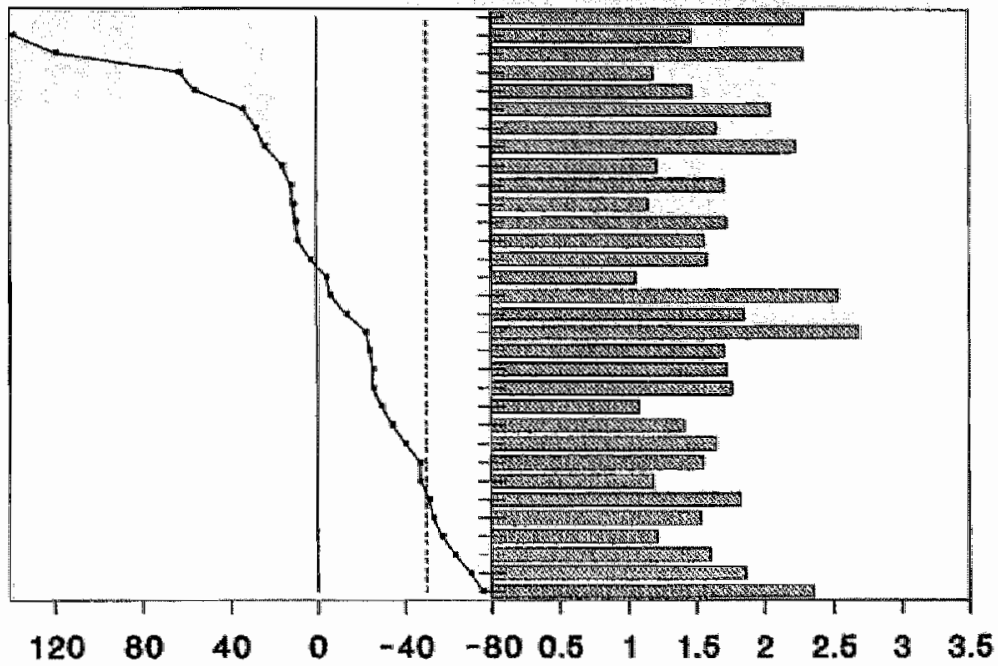

Fig. 5.3.e. Plasma concentrations and efficacy of loredezole.

belonged to the active substance group; in 1 of them it was carbamazepine that was reduced and in 2 it was phenytoin. Except these 3 patients, 7 other patients reported adverse events.

Somnolence occurred in 1 patient in the active substance group and 1 patient in the placebo group; similarly, mild headache occurred in 1 patient in each group. There was mild nausea and itchy eyes in 1 patient and some loss of appetite in another, and mild fatigue in 1 patient, although this could not be ascribed with any certainty to the lorecle. zole medication. One patient receiving placebo complained of irritability (Fig. 5.3.f). Analysis of the biochemical and haematological parameters showed that there were no abnormal values or changes in any of the parameters measured. Before inclusion in the study 50 of the patients had already elevated $\gamma$-GT concentrations, attributed to the use of the antiepileptic medication. The patient who was not taking any other medication had normal $\gamma$-GT values before and during the study. In 65 patients there was a further sight increase in this value during the loreclezole treatment.

\section{4}

Discussiion

To permit a more reliable comparison between placebo treatment and active lreatment, patients were selected on the basis of the number of seizures in the baseline period. This prevented a situation in which the groups were found to be noncomparable at the end of the study because of differences that had existed at the start of the study. Despite these precautions the active treatment group had, on average, somewhat more seizures than the placebo group $(0.44$ and 0.35 seizures per day respectively). In both groups the 

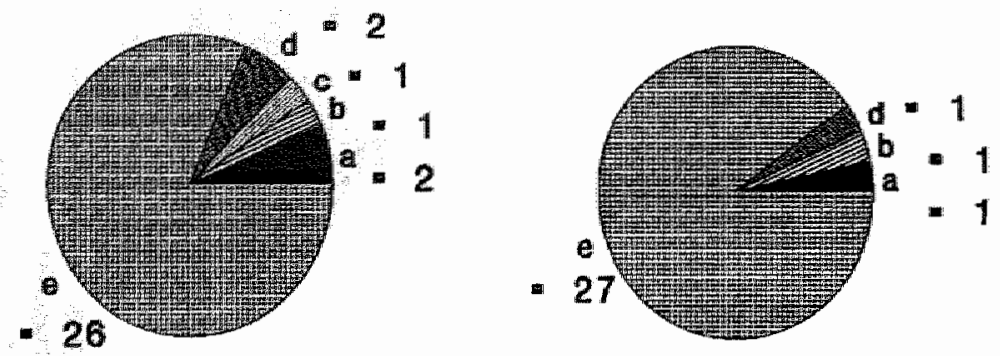

Loreclezole group $(n=5 / 32)$ Placebo group $(n=2 / 30)$

Fig. 5.3. Adverse events. $a=$ sleepiness/fatigue $b=$ headache, $c=$ mausea, $d=$ other, $e=$ none

patients had at least 4 seizures per month, but some patients had very many more. A patient receiving active treatment was therefore matched with a placebo treated patient who initially had the same seizure frequency.

The initial loreclezole dose was sufficient to produce a plasma concentration of $1-2 \mathrm{mg} / \mathrm{l}$ within 14 days in most patients. In most patients, however, a maintenance dose of 25 mg/day proved insufficient to maintain this plasma level. This is discussed in the section on loreclezole dosage in volunteers and induced patients (Section 8.5). In the placebo group an improvement of more than $40 \%$ was observed in 1 patient. In the active treatment group there were 6 patients who obtained a seizure reduction of $47-76 \%$. The patients with insufficient seizure reduction had plasma loreclezole concentrations comparable to those of the patients who obtained a seizure reduction of $50 \%$ or more, and the plasma concentrations of the existing antiepileptic drugs remained unchanged.

In contrast to previous studies, adverse events were reported, in the form of somnolence, whilst dizziness, double vision and ataxia in 3 patients were attributed to carbamazepine and/or phenytoin plasma concentrations that had become raised, despite maintenance of constant daily dosages. Adjustment of the dosages of these drugs to restore the plasma concentrations that had existed at the start of the study resulted in rapid disappearance of the symptoms. Somnolence occurred in 1 patient in each of the 2 groups. Some vague symptoms that could not be properly identified were seen in both groups. Loreclezole was tolerated well in all cases.

\section{Conclusion}

Although monotherapy is preferable in epilepsy treatment, polypharmacy is widely used in pharmacotherapy-resistant patients and is regarded as inevitable. In this double-blind placebo-controlled study, in which loreclezole or placebo was added to the existing antiepileptic medication, there were only 7 patients on monotherapy and 1 patient who had no existing medication at all. The dosage scheme began with at least 10 days on a loreclezole dose of $100 \mathrm{mg} / \mathrm{day}$, which if necessary was then adjusted on the basis of 
plasma concentration. The dosage scheme was sufficient to reach the target plasma concentration of about $1 \mathrm{mg} / \mathrm{l}$, but on chronic administration the plasma concentration was found to fall, so that intermediate dosage increments had to be introduced.

Significant interaction occurred only with clobazam; adjustment of the dosage was unnecessary. In individual cases there were interactions with carbamazepine and phenytoin.

The occurrence of changes in seizure frequency in intractable epilepsy patients, not only during active treatment but also during placebo treatment, is a familiar phenomenon in epilepsy treatment (Wilenski 1990). In the group treated with loreclezole there were 6 patients who obtained a seizure reduction of $47-76 \%$, whereas none of the placebo treated patients reached this percentage. Mild adverse events, particularly sedation and headache, were reported in both groups, but did not necessitate modification of the treatment in any of the patients.

As in the open add-on study reported in Chapter 3 , the loreclezole dosage in this doubleblind placebo-controlled study was limited to a plasma concentration of about $2 \mathrm{mg} / \mathrm{l}$. It is possible that a higher loreclezole dosage might have had an effect in a number of nonresponders. The protocol prohibited a higher loreclezole dosage, however. 


\section{Chapter 6 ONE-YEAR EVALUATION OF THE EFFICACY AND SAF- ETY OF LORECLEZOLE FOLLOWING THE DOUBLE- BLIND PLACEBO-CONTROLLED STUDY*}

\section{1}

\section{Introduction}

The patients who had taken part in the double-blind placebo-controlled parallel study of the efficacy and safety of loreclezole were given the opportunity to continue with the treatment if they had been taking loreclezole, or to start loreclezole treatment if they had been taking placebo (Chapter 5). The aim was to study the safety and efficacy of loreclezole in the long term and to investigate the interaction of the substance with existing antiepileptic drugs. A further aim was to investigate the effects of loreclezole at higher dosages, if such doságes became necessary.

\section{Methods and patients}

Of the original group of 62 patients, who were admitted to the double blind trial (Chapter 5) 56 patients elected to participate in this long-term follow-up study, 29 from the original toreclezole group and 27 from the placebo group. They fulfilled the inclusion criteria that had been stipulated for the double-blind placebo-controlled study (Chapter 5 ), except of course for the requirement concerning the minimum frequency of seizures.

The patients who had not previously taken loreclezole (i.e. who had been treated with placebo in the previous study) were given loreclezole in accordance with the same dosage scheme that had been used in the double-blind study: $100 \mathrm{mg}$ per day for about 10 days, followed by dose adjustments as described in Section 5.3.1, depending on the plasma concentration on days 7 and 14. The seizure frequency was evaluated each month and, in the event of insufficient efficacy, the loreclezole dose was raised to a level corresponding to a plasma concentration of about $5 \mathrm{mg} / \mathrm{l}$.

In the patients who had already received loreclezole in the previous study the loreclezole dose was raised, depending on the success of the treatment, by an increment of $\mathbb{I} \mathrm{mg} / \mathrm{l}$ in plasma concentration every 3 months, until a plasma concentration of about $5 \mathrm{mg} / \mathrm{l}$ was reached.

In this study, just as in previous studies, the dosage of the comedication was kept constant. At predetermined times the plasma concentrations of loreclezole and the comedication were determined and comprehensive haematological and biochemical investigations were performed (Table 6.2.a). On fixed monthly dates the seizure frequency and adverse events were evaluated by the investigator with the patient on the basis of diaries and the loreclezole dosage was adjusted if necessary. During the run-in phase that applied to the ex-placebo users the patient was examined weekly by the investigator.

\footnotetext{
* The results of this sudy are published as: Rentmeester T. Janssen A, Hulsman X. Scholtes F, Van der Kleij B, Owerweg J, Meijer J. De Beukelaar F. Long-term evalwation of the efficacy and safety of lorechezole as add-on therapy in patients with uncontrolled partial seizares: a 1-year open follow-up. Epilepsy Res 1991: 9:65-70
} 
As in the previous studies, the effect was evaluated by examining the absolute seizure frequency without considering any changes in seizure type. Statistical analysis in this study was the two-tailed Wilcoxontest for inter-group comparison as descibed in Section 5.2.4.

- plasma concentrations of loreclezole and other antiepileptic drugs, including metabolites and any free fractions

- haematological investigations: red and white blood cells, platelets, haemoglobin, haematocrit, differential blood count

- biochemical investigations: glucose, urea, creatinine, uric acid, cholesterol, bilinbin, ASAT, ALAT, $\gamma$ GT, LDH, alkaline phosphatase, folic acid, sodium, potassium, calcium, chloride, phosphate, total protein, albumin

Table 6.2.a. Laboratory determination

\section{3}

Results

\section{3 .1}

Plasmo concentrations

At the end of the study, i.e. after 1 year of loreclezole treatment, the 2 groups showed comparable mean plasma loreclezole concentrations: $4.6( \pm 1.6) \mathrm{mg} / \mathrm{l}$ in the group that had previously received loreclezole and $4.6(\$ 1.7) \mathrm{mg} / \mathrm{l}$ in the group that had previously received placebo. During the long-term treatment, a decrease in carbamazepine concentrations accompanied by an increase in the dihydroxycarbamazepine/carbamazepine ratio was observed. The carbamazepine-10,11-epoxide/carbamazepine ratio was not changed. In 11 out of 22 patients who were on phenytoin, dose reduction of this drug was carried out in order to keep concentrations during loreclezole treatment as close as possible to pretreatment values. However, if changes of greater than $25 \%$ from baseline in either direction are considered as significant, 10 patients showed an increase in phenytoin plasma levels whereas 2 presented a decrease at the end of 1.2 months' treatment (Table 6.3.a).

\begin{tabular}{lllll|}
\hline AED & $\mathrm{n}$ & base & $6 \mathrm{~m}$ & $12 \mathrm{~m}$ \\
$\mathrm{CBZ}$ & 45 & $6.82 \pm 1.60$ & $5.31 \pm 1.98$ & $4.93 \pm 1.66$ \\
$\mathrm{epx} / \mathrm{CBZ}$ & 38 & $0.35 \pm 0.20$ & $0.39 \pm 0.24$ & $0.39 \pm 0.21$ \\
diol/CBZ & 38 & $0.43 \pm 0.18$ & $0.80 \pm 0.40$ & $0.83 \pm 0.34$ \\
$\mathrm{PHT}$ & 22 & $14.0 \pm 3.05$ & $17.6 \pm 5.43$ & $16.4 \pm 5.10$ \\
\hline
\end{tabular}

Table 6.3.a. Plasma antiepilepric drug concentrations and metabolic ratios after 6 and 12 months of weatment with loreclezole (mean \pm S.E.). base = baseline value. CBZ = carbanazepine (mg/l). epx $=C B Z-10,1$-epoxide, diol $=$ dihydroxy-CBZ. PHT $=$ phenytoin $(\mathrm{mg} / \mathrm{l}), C L B=$ clobazam $(\mathrm{ng} / \mathrm{l}), \mathrm{DMC}=$ desmertylclobazam, $6 \mathrm{~m}=$ value at 6 months, $12 \mathrm{~m}=$ walue at 12 months. 
Starting from the mean seiztre frequency of 0.44 seizures per day in the baseline period, the group that had previously received loreclezole obtained a further reduction in mean seizure frequency to 0.28 seizures per day on treatment with higher loreclezole doses. The group that had previously received placebo had a seizure frequency of $0.35(0.05-$ 2.14) seizures per day before the treatment, which fell to $0.24(0.02-1.36)$ seizures per day at the end of the loreclezole treatment. In the double-blind placebo-controlled study, 6 of the patients receiving loreclezole had had a reduction of more than $50 \%$ in seizure frequency. In the present 1-year study the number of patients with a seizure reduction of more than $50 \%$ rose to 22 of the 56 patients in the 2 groups after addition of loreclezole to the existing antiepileptic medication (Fig. 6.3.b).

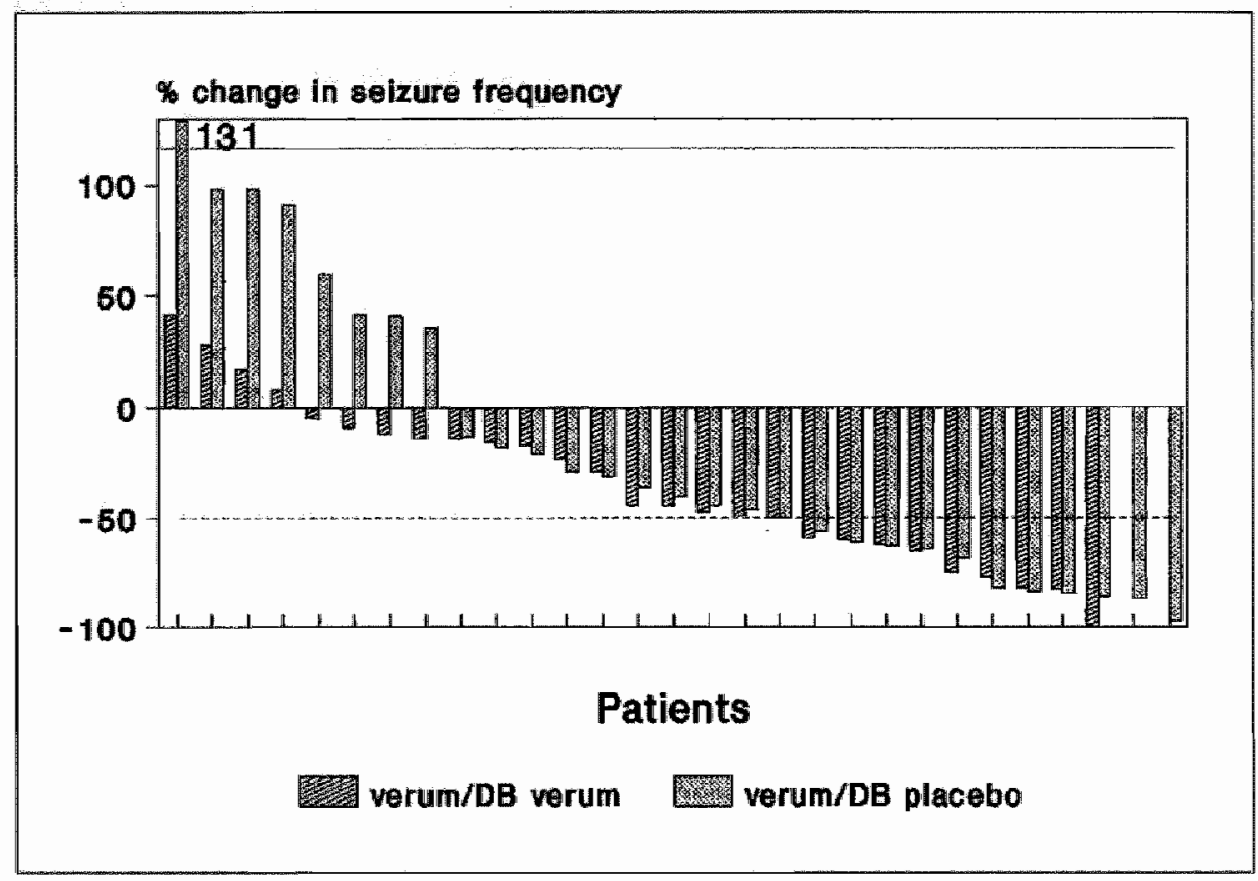

Fig. 6.3.b. Eftacty of loreclezole after 1 year treament. DB indicates treament in double-blind phase (Ch. S)

Referred to baseline, the reduction in seizure frequency was significant after 6 months $(p<0.0001)$ and 12 months $(p=0.0001)$ of loreclezole treatment. Seventeen of the 56 patients had a seizure reduction of $25-50 \%$. There were 9 patients who experienced an increase $(2-75 \%$, mean $36.5 \%)$ in seizure frequency during loreclezole treatment.

\subsubsection{Adverse events}

No serious adverse events were reported during the loreclezole treatment. Adverse events occurred in 27 patients. There were a few reports of somnolence and fatigue ( $\mathrm{n}=$ $6)$, dizziness $(n=1)$, ataxia and double vision $(n=2)$, gynaecomastia $(n=2)$, akinesia 


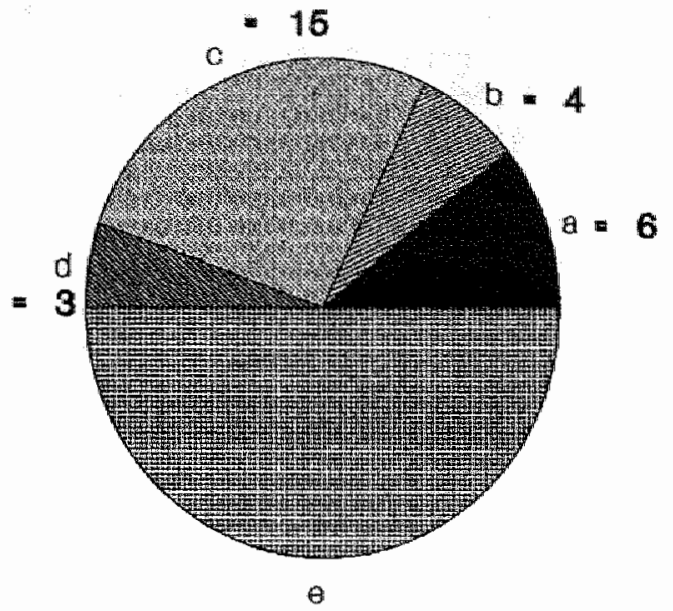

- 29

Fig. 6.3.c. Adverse events $(n=27 / 56$ patients), $a=$ sleepiness, $b=$ ataxia $/$ diplopia $c=$ werght loss, $d=$ other, $e=$ none

$(\mathrm{n}=1)$ and weight loss $(\mathrm{n}=15)($ Fig, 6.3.c). One of the patients with gynaecomastia, 1 patient with fatigue, and 1 patient with weight loss stopped the loreclezole treatment for these reasons. Thyroid function was investigated in all 15 patients with weight loss; none of them showed changes with respect to baseline. In the group as a whole the mean of the body mass indices remained normal: $23.7 \mathrm{~kg} / \mathrm{m}^{2}\left(15.9-29.1 \mathrm{~kg} / \mathrm{m}^{2}\right)$ before administration of loreclezole and $21.1 \mathrm{~kg} / \mathrm{m}^{2}\left(14.9-24.5 \mathrm{~kg} / \mathrm{m}^{2}\right)$ at the end of the studly.

6.4

Discussion

The double-blind placebo-controlled study (Chapter 5) showed that, at dosages from $12.5 \mathrm{mg} / \mathrm{day}$ (about $0.2 \mathrm{mg} / \mathrm{kg} / \mathrm{day}$ ) to $100 \mathrm{mg} / \mathrm{day}$ (about $1.4 \mathrm{mg} / \mathrm{kg} / \mathrm{day}$ ) and plasma concentrations of $1.05-2.69 \mathrm{mg} / \mathrm{l}$, loreclezole is safe and effective in the treatment of seizures in patients with pharmacotherapy-resistant epilepsy. In the present follow upstudy, loreclezole was given to patients who had received placebo in the previous study and the loreclezole treatment, at a higher dosage if necessary, was continued in patients who had previously received loreclezole and had benefited from it.

The patients who were receiving loreclezole for the first time were initially adjusted to the same plasma concentration as in the double-blind study (Chapter 5), and were then - like the patients who had already received loreclezole - gradually given a higher dosage until the effect was adequate (at plasma concentrations of about $5-10 \mathrm{mg} / \mathrm{l}$ ).

In this study too loreclezole was well tolerated; only sporadically were there reports of mild adverse events such as transient somnolence and weight loss. Loreclezole was effective against epileptic seizures. Averaged over the trated population as a whole, the seizure frequency fell from 0.40 to 0.26 seizures per day. Thirty-one of the 56 patients 
had a seizure reduction of more than $25 \%$ and 22 of these had a seizure reduction of more than $50 \%$, whilst only 9 had an increase $(2-75 \%)$ in seizures. The effect was evaluated solely on the basis of the number of seizures. A number of patients reported an effect not only on the number of seizures but also on the severity of the seizures. Thus an effect regarded as favourable by the patient, such as the disappearance of tonic-clonic seizures or of status epilepticus, is not reflected in the results.

On average, a plasma concentration of $4.6 \mathrm{mg} / \mathrm{l}$ was reached during loreclezole treatment. Carbamazepine plasma concentration decreased slightly, most probably as a result of an accelerated metabolism due to liver enzyme induction. Since there was no disproportional increase in the carbamazepine-10,11-epoxide/carbamazepine ratio, it is suggested that the epoxide- and hydroxylation metabolic pathways of carbamazepine were stimulated to the same extent. The absence of an increase in carbamazepine-10,11epoxide concentration is of great importance as this metabolite is considered to be a pharmacologically active but also toxic entity (Section 1.4.4.). This interaction is therefore is of smaller relevance in the interpretation of the results. In 10 patients a $25 \%$ or greater increase in phenytoin plasma concentration was found. The question may be raised as wether this increase influenced the results in favour of the test drug. However in 7 of these patients the elevated phenytoin plasma concentration did not result in a $50 \%$ or greater decrease in seizure frequency. One patient responded by the end of 12 months' treatment with loreclezole but had not responded by 6 months although phenytoin plasma concentrations were higher at this point than at 12 months. The beneficial effect is unlikely to be attributed to phenytoin. In two patients a possible effect of the slightly higher phenytoin plasma concentrations cannot be ruled out. However, in these patients higher phenytoin plasma concentrations in the past had not resulted in lower seizure frequency.

\section{5}

\section{Conclusion}

Patients who had taken part in the double-blind placebo-controlled parallel study were treated further with loreclezole. The existing antiepileptic therapy was kept unchanged during the treatment. Depending on the treatment results, loreclezole was dosed to a plasma concentration of approximately $5 \mathrm{mg} / \mathrm{l}$. In $42 \%$ of the patients a reduction of $50 \%$ or more in seizure frequency was attained. There were no adverse events requiring modification of the treatment. Interaction with carbamazepine and clobazam seems to be of no consequence. It is unlikely that increase of phenytoin plasma concentration has been of relevance in the results of the study. Nevertheless, this observation compels to proceed with caution.

This study confirms the efficacy and safery of loreclezole in the treatment of serious epilepsy. 


\section{Chapter 7. GRADUAL TRANSITION TO LORECLEZOLE MONO THERAPY}

\section{1}

\section{Introduction}

In modern epilepsy treatment monotherapy is vastly preferable (Parsonage 1980, Shorvon 1985). It has been shown that the majority of epilepsy sufferers (about $80 \%$ ) derive sufficient benefit from treatment with 1 drug (Schmidt 1982b). Combinations of antiepileptic drugs are seldom necessary. Polypharmacy leads more frequently and more rapidly to adverse events. The ultimate goal, freedom from seizures, is sometimes reached after withdrawal of comedication (DePew and Lubozynski 1989, Ramsay et al. 1989, Mirza et al. 1989, Salvato et al. 1989, Andersen and Pedersen 1989).

The loreclezole studies described in this study so far were concerned solely with add-on treatment. Once a new antiepileptic drug, like loreclezole has been added to the medication, the obvious next step is to make a gradual transition to withdrawal of the existing antiepileptic drugs (Wagner at al. 1991). Accordingly, as a follow-up to the studies that had already been performed - the open add-on study (Chapters 3 and 4 ) and the doubleblind placebo controlled parallel study (Chapters 5 and 6) - a protocol was drawn up for gradual transition to loreclezole monotherapy.

Patients who had taken part in the previous studies were eligible to take part in the present study. These were patients who had derived benefit from the loreclezole treatment. In assessing this benefit, account was taken not only of the percentage reduction in seizures but also of the shift in seizures from day to night. A reduction in the severity of the seizures, sufficient to be regarded as an improvement by the patient, was also acceptable as a reason for including patients in the study, even if there had been no reduction in seizure frequency. The aim of the study was to investigate whether loreclezole is effective against epilepsy when given as monotherapy.

\section{2}

\section{Methods and patients}

Before reducing the comedication in patients who were eligible for the study, the plasma concentration of the antiepileptic medication was determined during the day: blood samples for this purpose were taken every $2 \mathrm{~h}$ between $8.00 \mathrm{a} . \mathrm{m}$. (fasting) and $6.00 \mathrm{p.m}$. The findings were tised to check for fluctuations in the platsma concentrations of the medication used (Duncan et al. 1991).

In principle the patients were to be treated as outpatients; they were to be hospitalized only if there was a compel ing indication for hospitalization. The rate of reduction of medication proposed by Newmark - immediate discontinuation of antiepileptic drugs was rejected because it requires hospitalization (Newmark 1990). The slow reduction of concomitant antiepileptic drugs, proposed by Schmidt - i.e. $25 \%$ dose reduction every 2 months, also was rejected, in view of the clinical improvement experienced by the patients after addition of loreclezole to their medication (Schmidt 1985). Thus the rate of reduction of medication was comparable to what was referred to by Duncan et al. as the slow scheme and was described by Overweg in seizure-free patients (Overweg 
1985, Duncan al al 1990). The drug which, in the opinion of the investigator, was probably the least effective or induced the most adverse events was withdrawn first (Dean and Penry 1989). In principle the medication was reduced in accordance with a fixed scheme and was finally withdrawn altogether (Table 7.2.a).

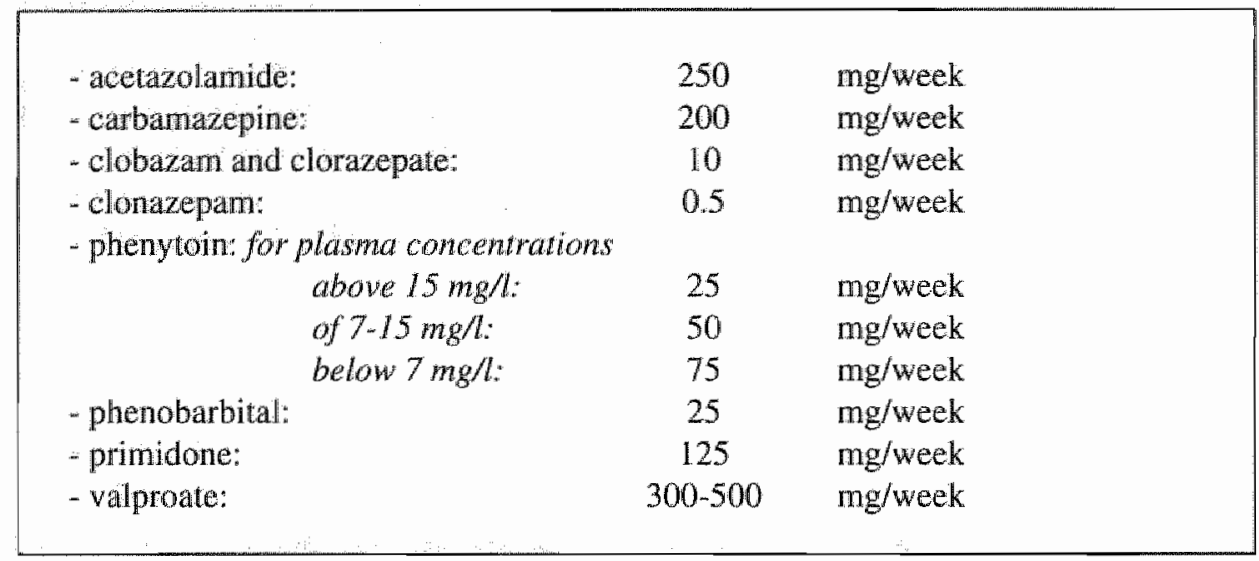

Table 7.2.a. Medication reduction scheme: drug (in alphabetical order) and reduction in total daily dose. which was then maintained for I week.

Sometimes, for the sake of the safety of the patients or for other special reasons, the medication was reduced more slowly. After 1 antiepileptic drug had been completely withdrawn in accordance with this scheme, the medication was kept constant for at least 2 months. If the patient suffered an intolerable increase in seizures the old medication was restored. Plasma loreclezole concentrations were kept between 5 and $10 \mathrm{mg} / 1$.

After the 2 months of constant medication the next antiepileptic drug was withdrawn in accordance with the same scheme, its eventual total withdrawal being again followed by a 2 -month "rest" period, until monotherapy with loreclezolle was attained. During the withdrawal of the comedication, possible changes in haematological and biochemical parameters were investigated as in the previous studies (Table 7.2.b). Once monotherapy had been neached, these determinations were limited to one every 3 months. The evaluation period was 6 months.

- plasma levels of loreclezole, other antiepileptic drugs, degradation products and any liee fractions

- haematology: haemoglobin, haematocrit, leucocytes, ery throcytes, platelets and differential blood count

- biochemical investigations: glucose, urea, creatinine, cholesterol, bilirubin, ASAT, ALAT, $\gamma$ GT, LDH, alkaline phosphatase, folic acid, sodium, potassium, chloride, calcium, phosphate, uric acid, total protein and albumin

Table 7.2.6. Laboratory determination. 
During the study there were frequent (at least once every 2 months) evaluations of seizures and any adverse events, together with neurological examinations.

\section{2 .1}

Inclusion and exchusion criteria

Both men and women who had participated in one of the studies of the efficacy and safety of loreclezole described in this thesis were eligible for participation in the present study. As some patients had become seizure-free during the preceding loreclezola treat-

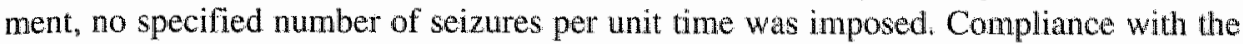
therapy remained necessary, of course.

In view of the limited number of patients suitable to the trial 2 patients with pharmacotherapy-resistant localization-related epilepsy - and considered to treatment with loreclezole - were added and treated in accordance with the protocol. In the 2 newly included patients loreclezole was added to the existing medication in accordance with the dosage scheme described in Section 5.2.2. Patients with hypersensitivity to loreclezole or with serious side effects of loreclezole were excluded from the study. Further exclusion criteria were pregnancy or risk of pregnancy, progressive neurological diseases, serious liver or kidney dysfunction, and absence of written or oral informed consent.

Nine patients were evaluable in this study: 3 patients $(0704,0709$ and 0712) from the studies described in Chapters 3 and 4,4 patients (1410, 1412, 1413 and 1416) from the double-blind placebo-controlled study (Chapters 5 and 6) and 2 patients (patient $\mathrm{ml}$ and $\mathrm{m} 2$ ) who had not previously used loreclezole. Comedication was reduced in accordance with the scheme. In addition to loreclezole, 8 patients were taking 1 antiepileptic drug 6 $\times$ carbamazepine, $2 \times$ phenytoin) and 1 was taking 2 (carbamazepine and phenytoin). Seven patients were using loreclezole monotherapy at the end of this study.

Results

The gradual withdrawal of the comedication was tolerated well by the patients; no con plications arose during withdrawal.

\subsubsection{Efficacy}

The results of the withdrawal of the antiepileptic comedication in this group of patients are shown in Fig. 7.3.a. Solely during reduction of medication, patient 0709 had a shatp increase in seizure frequency from 0.4 to 1.2 seizures/day, but no modification of treatment was necessary. On reaching monotherapy, 2 patients $(0712$ and 1410$)$ experienced a temporary deterioration in the sense of a partial status epilepticus and a series of com. plex partial seizures. In patient 1410 this did not require extra medicinal support. In patient 0712 intravenous administration of phenytoin and clonazepam proved ineffective. In both cases there was a spontaneous and complete recovery. The patients continued treatment with monotherapy. Four patients had an irrelewant change in seizure frequency after withdrawal of the comedication, 1 patient (1416) had an increase in the 
severity of the seizures, and 2 patients (0704 and $\mathrm{m} 2$ ) had a clear increase in seizure frequency. In both cases, valproate was added to the loreclezole monotherapy, resulting in restoration of the original seizure frequency. In patient $\mathrm{m} 2$ the severity of the seizures had decreased considerably and there was no longer secondary generalization of the partial seizures. In 2 patients (1412 and $\mathrm{ml}$ ) there was a clear reduction in seizure frequency (Fig. 7.3.a).

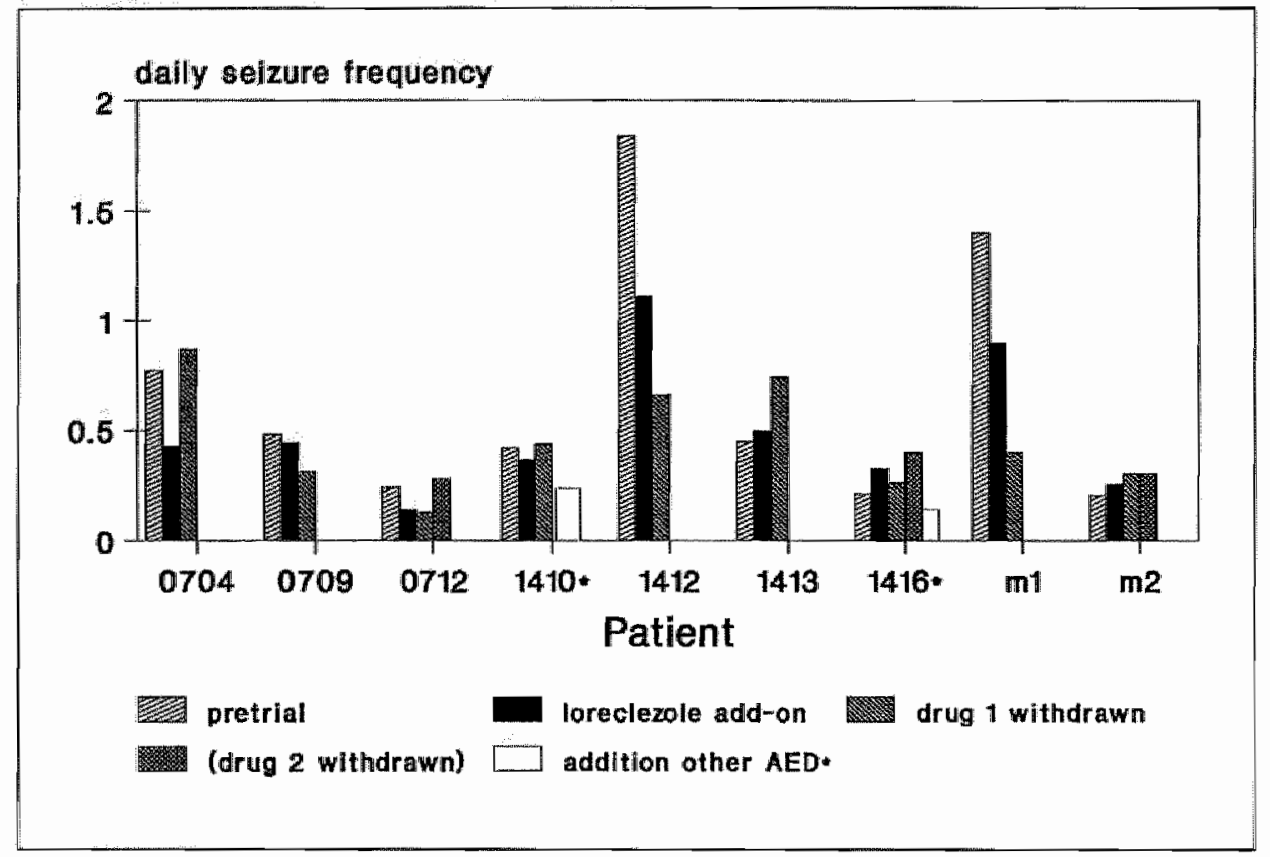

Fig. 7.3.a. Efficacy of loreclezole in monotherapy. Seizure frequency/4 weeks. Last bar is monotherapy, "except in 1410 and 1416 .

\subsubsection{Plasma concentrations}

Blood tests were performed at least every 2 months. Loreclezole plasma concentrations remained between 4 and $9 \mathrm{mg} / \mathrm{l}$ in all patients. On the whole, loreclezole was tolerated. well. Some patients reported that they felt fitter and more alert on monotherapy. This was not investigated further, e.g. by neuropsychological investigation.

\subsubsection{Adverse events}

During this study there were no adverse events that had not already been reported by the patients during the previous studies. Any existing weight loss stabilized. None of the patients had side effects requiring a change of treatment.

Discussion

In the first instance, loreclezole was added to the medication of hitherto pharmacotherapy-resistant epilepsy patients selected because their seizure frequency was hight despite 
treatment with all conventional drugs. Those who benefited from addition of loreclezole to the existing treatment were offered the opportunity to reduce the comedication, in accordance with the protocol, until they were taking loreclezole monotherapy. Nine of the patients who took part in the study were evaluable. In 1 patient, reduction of the comedication was associated with a temporary increase in seizure frequency but produced no further complications. Two patients eventually requested addition of another antiepileptic drug to their medication again, 1 on account of an increase in frequency and the other because the seizures had become more serious. Two patients had an increase in seizure frequency without requiring additional medication. Two patients had a clear decrease in seizure frequency with respect to the polytherapy.

Not mentioned among the participants in this study is patient 1401 from the studies described in Chapters 5 and 6, who was already using loreclezole monotherapy.

\subsection{Conclusion}

Monotherapy is preferable in the drug treatment of epilepsy. Accordingly, an antiepileptic drug is truly of value only if it can be used as monotherapy. The study described in this chapter shows that loreclezole is an effective antiepileptic drug in monotherapy too, even in patients suffering from serious forms of hitherto pharmacotherapy-resistant epilepsy. This opens the way to treatment of newly diagnosed patients with loreclezole in comparative studies versus conventional antiepileptic drugs. The efficacy of loreclezole in monotherapy should be investigated more closely. 


\title{
Chapter 8. EVALUATION OF THE EFFICACY AND SAFETY OF LORECLEZOLE
}

\section{1}

\author{
Introduction
}

Section 1.1 describes the necessity for the development of new antiepileptic drugs. Alhough modern antiepileptic drigs have acceptable efficacy in many patients, a considerable number of patients derive insufficient benefit from their medication. Only a small proportion of these patients are suitable for surgical treatment of their epilepsy. In addition there are patients who do obtain effective seizure control from their existing medication but suffer from its side effects. So there is a need for more effective and safer antiepileptic drugs. Loreclezole, a member of the triazole group, was developed as. a new antiepileptic drug. Animal findings had shown that loreclezole should be effective in the treatment of epilepsy. Since then, various studies have confirmed that loreclezole is also effective against epileptic seizures in humans (Chapters 3-7). This chapter discusses some aspects of loreclezole that are important in the treatment of epilepsy patients.

\section{2}

\section{Efficacy of loreclezole}

Animal experiments have shown that a plasma concentration of 1-2 mg/l gives effective protection against convulsions (Section 1.5.2). The effect of plasma concentrations of about $2 \mathrm{mg} / \mathrm{l}$ is favourable in a number of patients (Chapter 3 ): 4 (31\%) out of 13 patients obtained a seizure reduction of $50 \%$ or more. The same effect - a seizure reduction of $50 \%$ or more was obtained in $6(19 \%)$ of the 32 patients in the double-blind placebo-controlled parallel study (Chapter 5). When the loreclezole dose was raised to give a plasma concentration of about $5 \mathrm{mg} / \mathrm{l}$, the number of patients whose seizure frequency was reduced by at least $50 \%$ rose to $22(40 \%)$ of the 56 patients (Chapter 6$)$. It should be noted that the patients in this group had a long history of hitherto pharmacotherapy-resistant epilepsy, i.e. that there had been negative selection of patients who are particularly resistant to improvement (Gram et al. 1982). A number of patients reported a reduction in the severity of the seizures. There is no simple way of measuring this improvement (Section 1.6.2). The majority (72 out of a total of 75) of the patients who took part in these studies were surfering from epilepsy with partial seizures with complex symptomatology, 25 of them with secondary generalization, whilst there were only 3 patients with symptomatic generalized epilepsy. In these studies loreclezole was not administered to sufferers from generalized idiopathic epilepsy, and so no conclusions can be drawn trom this study with regard to its efficacy in primary tonic-clonic seizures and absences. Animal findings suggest that loreclezole would indeed be of value in these forms of epilepsy, but clinical research is necessary to confirm this (Wauquier et a.l. 1990 ).

\section{3}

\section{Side effects}

Loreclezole was tolerated well during the studies. In both the open and the placebo-controlled study there were adverse events, which were predominantly mild. Placebo 
seemed to produce adverse events just as often as loreclazole. The side affect of nausea was caused in one case by intolerance of capsules, the dosage form of loreclezole in these studies. Dizziness, somnolence and ataxia were more common at plasma concentrations above $10 \mathrm{mg} /$. It seems appropriate to advise against plasma concentrations exceeding $10 \mathrm{mg} / 1$, although patients do exist who tolerate higher concentrations well (Engelsman et al. 1990). This is a familiar phenomenoi, however: so-called dherapeutic windows are reference values which apply to the majority of patients but have no absolute validity. As yet there is no explanation for the weight loss of about $10 \%$ reported by a number of patients $(n=18=25 \%$ ). The study group showed no biochemical changes. Supplementary investigations of the thyroid parameters T4 and T3 and the pituitary parameter TSH were performed in this group. There was no evidence of hyperthyroidism.

\section{4}

\section{Plasma concentrations}

In the treatment of epilepsy with antiepileptic drugs, the determination of plasma concentrations is very important (Meijer 1991).

In Chapter 3 it was mentioned that $1.2 \mathrm{mg} / \mathrm{l}$ is a value associated with a measurable favourable clinical effect (reduction of seizure frequency) in some patients with pharmacotherapy-resistant epilepsy. In the following studies the loreclezole dosage was raised to give plasma concentrations of initially $5 \mathrm{mg} / \mathrm{l}$, then $7 \mathrm{mg} / 1$ and, in some patients, finally about $10 \mathrm{mg} / \mathrm{l}$. This higher dosage was associated with a further improvement in seizute control in a number of cases and was mostly still well tolerated without adverse events.

Although the dosage scheme of the double-blind study (Chapter 5) gave the desired value of about $2 \mathrm{mg} / \mathrm{l}$ in the short term, adjustment was mecessary in most patients.. Nonlinear kinetics seem to be responsible. Between 1 and $2 \mathrm{mg} / \mathrm{l}$ saturation kinetics set in, leading to a disproportionate increase in plasma concentration above $2 \mathrm{mg} / \mathrm{l}$ as the dosage increases. The rapid and effective $(80 \%)$ absorption of loreclezole from the gastrointestinal tract permits a rapid increase in plasma concentration, but the long plasma half-life of 15-25 days hinders precise adjustment of the patient. The plasma half-life depends on the comedication used: enzyme-inducing antiepileptic drugs such as carbamazepine and phenytoin reduce the half-life to 7-14 days, whereas a mean half-life of 28 days is observed when just valproate is used. There is no evidence of autoinduction. Laboratory research has shown that plasma protein binding of loreclezole is about $99 \%$.

In view of the long plasma half-life (7-30 days) the plasma concentration may be expected to be stable over the day, with a slight peak after ingestion. This was investigated in 19 patients by determining the plasma loreclezole concentration between 8.00 a.m. (before ingestion of the medication) and 6.00 p.m. (Figs. 8.4.a and 8.4.b). These figures show that the fluctuation of the plasma concentration over the day is indeed slight. The consequences of this for the treatment are examined further in Section 8.5 .

When loreclezole is withdrawn a considerable period elapses before loreclezole clears from the blood. The time course of the plasma concentration after acute withdrawal of 


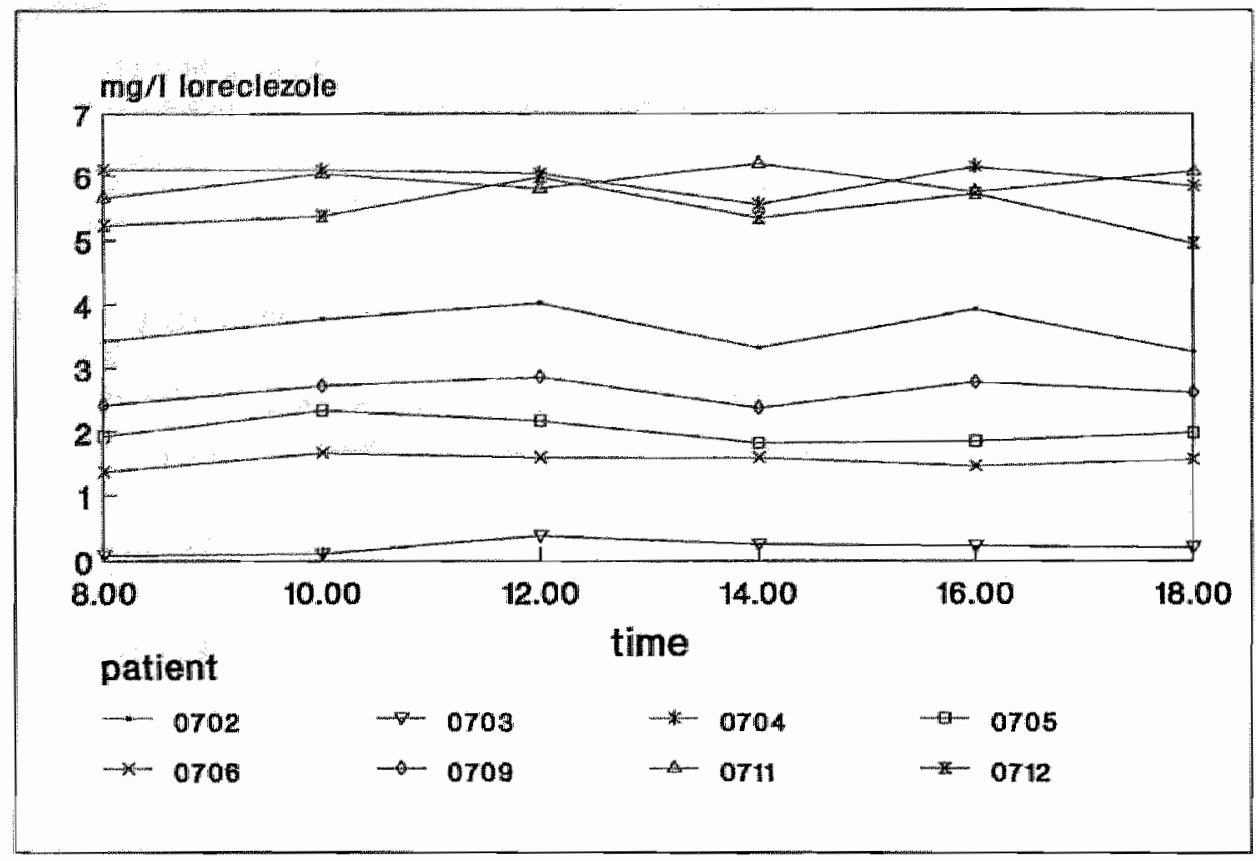

Fig. 8.4.a. Plasma concentrations of loreclezole between 8 a.m. and 6 p.m. Results from 8 patients.

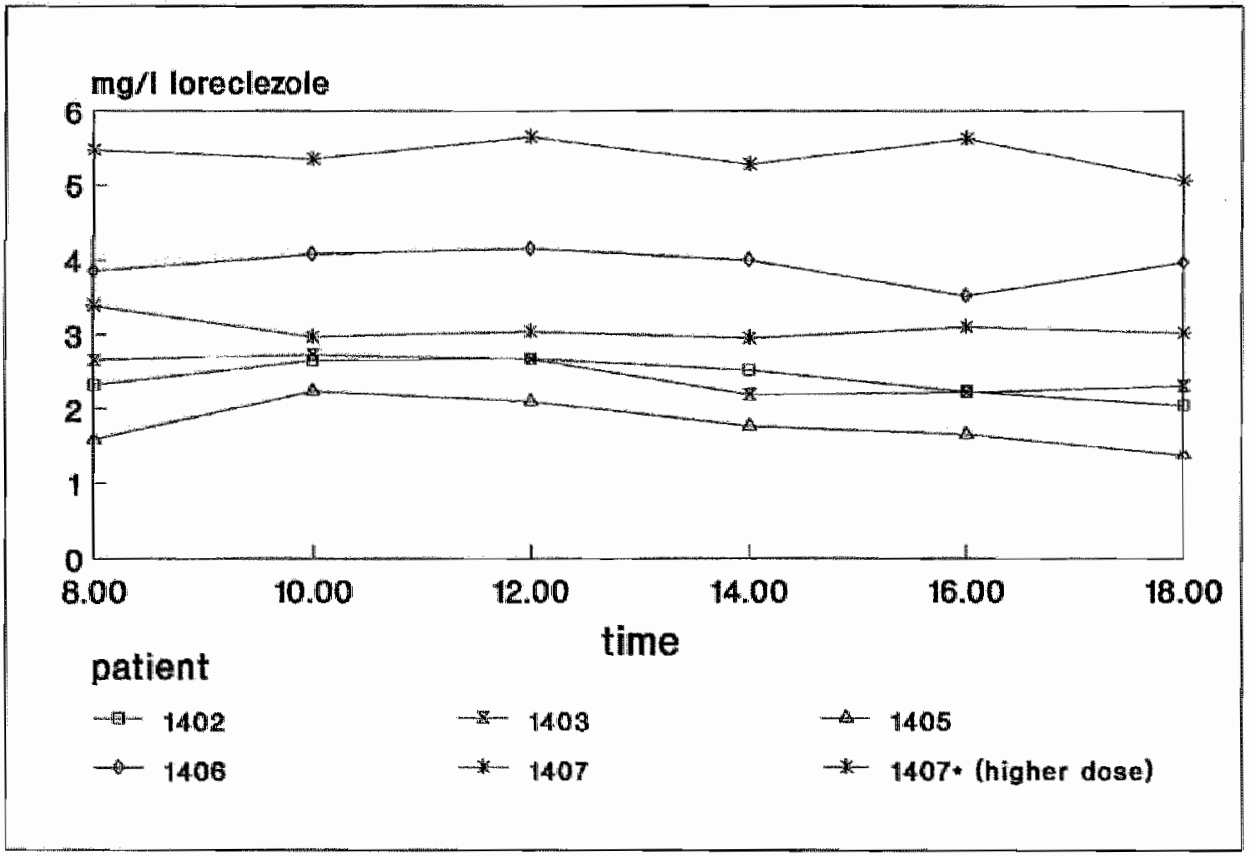

Fig. 8.4.b. Plasma concentrations of loreclezole between 8 am. and 6 p.m. Results from 5 patients. 
loreclezole was monitored in 10 patients. A kink was seen in the concentration/time curve at 1-2 mg/, just as had been seen during the build-up of the medication. The dosage or plasma concentration does not predict the speed of elimination, however (Figs. 8.4.c. and 8.4.d). The elimination rate varies widely from one individual to another.

Although loreclezole is detectable in saliva its concentration there is about 100th of its plasma concentration, and so this method of determination cannot yet be regarded as an alternative (Brunia et al. 1990).

Loreclezole dosage

In Chapter 3 (Section 3.3.1) it was stated that a single administration of a low dose of loreclezole $(50 \mathrm{mg}$ ) gives no indication of the chronic dosage that is necessary to give a plasma concentration of about $2 \mathrm{mg} / \mathrm{l}$. A mean dosage of $1 \mathrm{mg} / \mathrm{kg} /$ day gives a plasma concentration of $1-5 \mathrm{mg} / \mathrm{l}$, i.e. values that range from probably subtherapeutic to therapeutic. The risk of intoxication at this dosage is low. Determination of the plasma concentration one half-life time (Section 8.4) after introduction of $1 \mathrm{mg} / \mathrm{kg} / \mathrm{day}$ of loreclezole gives an indication of whether the patient is a rapid metabolizer requiring a high dose or whether a lower dose would be appropriate. Reassessment of the plasma concentration after about 4 weeks gives a clearer picture, allowing the dosage to be set with more certainty. Since loreclezole exhibits nonlinear pharmacokinetics the concept for reaching steady state in four times half-lives is not applicable (Benet 1990). In nonlinear pharmacokinetics steady state depends on $\mathrm{V}_{\max }$ and $\mathrm{K}_{\mathrm{m}}$ (Levy and Unadkat 1989). The long plasma half-life of loreclezole means that the dosage interval can be fairly long. A dosage scheme of once per day is tolerated well by all pattients, even at higher doses. As once daily dose regimen are prescribed, compliance improves dramatically (Eisen et al. 1990). Theoretically, 1 dose per week (i.e. minimum half-life time) should be sufficient to maintain an effective plasma concentration, but whether a very high single dose would be well tolerated requires further study (Fing】 1972). It is possible that an unacceptably high absorption peak might occur in such cases.

If it is known that the patient will be unable to take medication orally for a few days, e.g. after an operation, the period can be bridged by giving higher or multiple daily doses in advance. If an acute intervention becomes necessary or if the patient becomes unable for some other reason to take the medication when a dose becomes due, the omission of a few doses will not have serious repercussions: the plasma concentration falls only slightly in the short term and the protection given by the medication persists. Consequently, the introduction of a parenteral dosage form has low priority. For the treatment of status epillepticus might such a dosage form be useful.

After careful consideration the loreclezole treatment was stopped acutely in 10 patients in whom its effect was inadequate. In none of these cases did this lead to calamities. The seizure frequency that these patients had experienced before the loreclezole treatment, which in most of these cases was the same as the seizure frequency during the loreclezole treatment, continued unchanged after withdrawal of Joreclezole. Unlike the withdrawal of other drugs in patients who are not seizure-free, the acute withdrawal of loreclezole seems to present no problems in the sense of an excessive increase in seizu- 


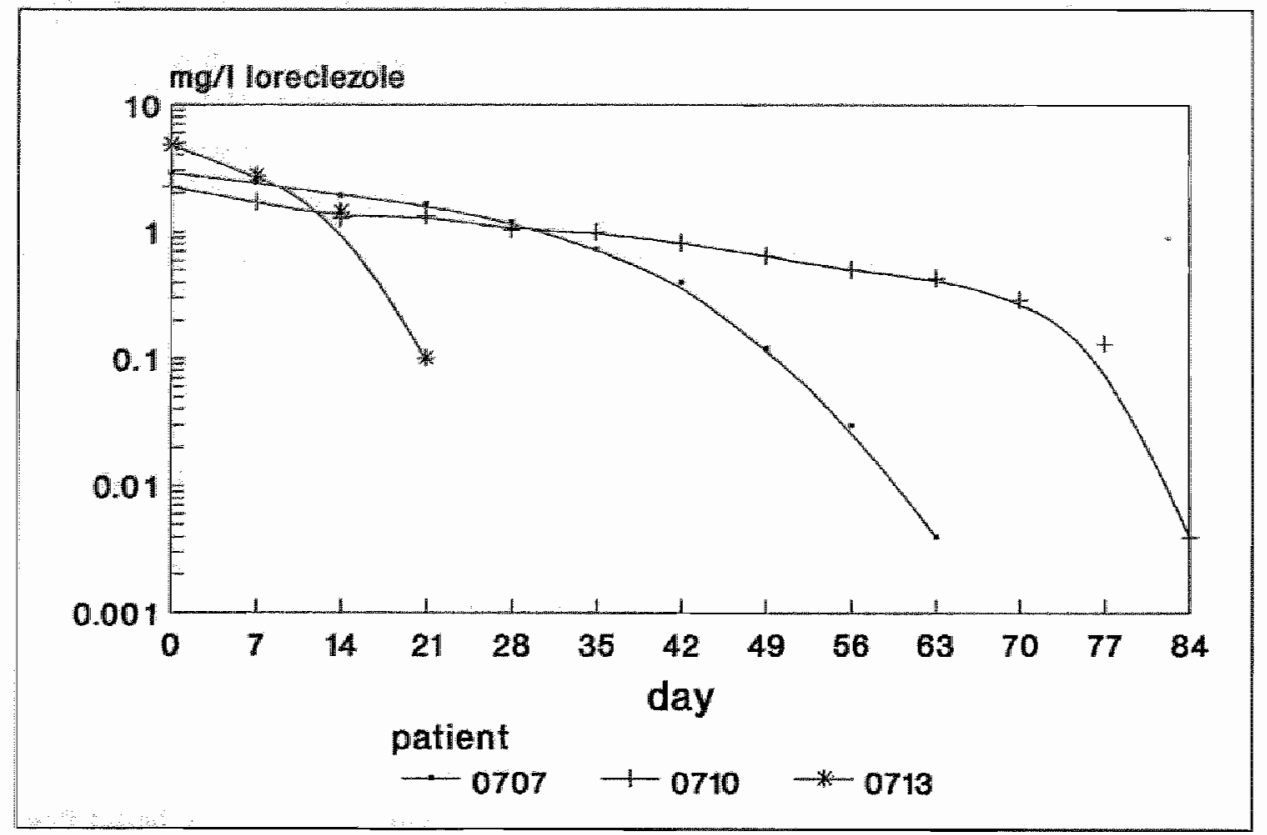

Fig. 8.4.c. Wash-out curves of plasma concentration of loreclezole. Resalts from 3 patients $1 \mathrm{Ch}$ 3.4).

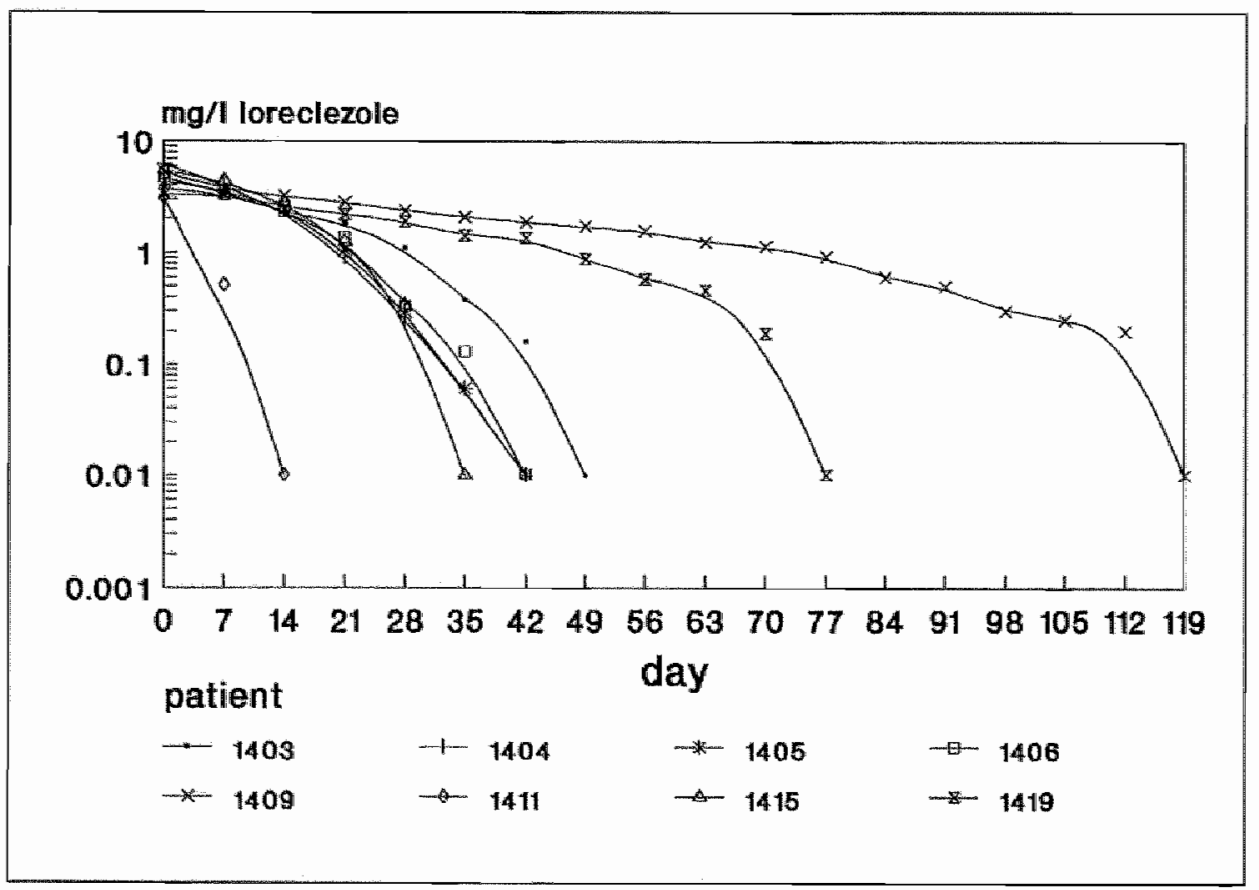

Fig. 8.4.d. Wash-ow cumes of plasma concentration of loreclezole. Results from 8 patients $(\mathrm{Ch} 5)$ 
res or in status epilepticus (Duncan et al. 1990). Whether or not this is also true in seinure-free patients is unclear from these observations. Like phenobubital, loreclezole has a long elimination half-life, but in the case of phenobarbital it is known that acute withdrawal can lead to serious complications, irrespective of whether the patient is free of seizures (Overweg 1985).

\section{6}

Methods of investigation

As mentioned in Section 1.6.1 there are several methods of investigation for new antiepileptic drugs (Porter 1985a). Once R 57720 and loreclezole have progressed through the antiepileptic drug development program in animals the drugs were introduced to healthy volunteers to prove safety and tolerability. Following these investigations the drugs were introduced to patients. According to the guidelines of the Commission on Antiepileptic Drugs of the ILAE, R 57720 and also loreclezole were first added to concomitant medication in patients for preliminary evaluation of the safety and efficacy and to determine the dose range within which the drugs can be safely tested in further studies (Commission on Antiepileptic Drugs 1989). Pharmacokinetics can also be evaluated in these studies, as well as drug-drug interactions. Knowledge of drug-drug interactions is mandatory for designing further studies, particularly in double-blind, placebocontrolled studies. The number of possible interactions is almost indefinite. Nevertheless reasonable choices can be made, taking into account combinations of drugs likely to be used together in daily practise (Sitsen 1991).

Initially R 57720 in the studies about this drug, was given as a single dose, in a longterm study with low dose, and followed by stepwise increase of the dose. The studies on loreclezole described in this thesis begin with a single dose study with a presumed efficacious dose to determine pharmacokinetics. Afterwards, it turned out that the dosages given to the patients were too low to predict the dose regimen in the following open add-on study. Due to non-linear kinetics of Joreclezole the dose of loreclezole in induced patients must be higher than in volunteers. Increase to the maximum tolerated dose of loreclezole was not given, because of the lack of adverse reactions in animals at protective dosages of $1-2 \mathrm{mg} / 1$ and higher. Increasing the dose to the maximum tolerated dose incorporate the risk of toxic effects at plasmalevels of loreclezole that are not necessary to reach maximum antiepileptic effect. However, the change in plasma halflife from some days to approximately 20 days during chronic treatment resulted in increasing and well tolerated plasmalevels, also resulting in better seizure control. These observations were not sufficient used in the set-up of the double-blind, placebo controlled study (Chapter 5). Otherwise at low dosages of loreclezole no important drug interactions were seen, whereas some beneficial effects were. This observation leads to the conclusion that loreclezole seems a safe antiepileptic drug even in severe cases of epilepsy. In these initial studies the recommended, small number of patients (about 10 ) were optimally used, first to investigate pharmacokinetics, and afterwards to evaluate safety and preliminary efficacy. In this way also the ethical considerations for using patients for investigational reasons were covered. Patients who benefit from the treatment with loreclezole, continued the use in a long term evaluation, and gradually withdrawal of concomitant antiepileptic medication. Preferably these studies are designed in a randomized fashion. Unfortunately the patients will get bored with study after study 
with potential risks, so cnly a small number of patients will be included. This was the reason for an open whitrawal desugn, in which the most toxic or least efficacious drug was withdrawn first. It is presumable that randomized withdrawal of concomitant antiepileptic drugs in all responders had led to more reliable results about efficacy of loreclezole in monotherapy, but also to several drop-outs. Spontaneous variation, in the case of increase in seizure frequency will stress the patient to violate the protocol by reverting to a previous medication.

In the double-blind, placebo-controlled study it turned out to be not necessary to adjust concomitant medication. Nevertheless in the follow-up studies with loreclezole interaction with phenytoin resulted in the necessity to change its dosage. In a double-blind setup this problem can be solved by adding concomitant medication in venum and dummy tablets. Another option in double-blind trials is randomized variation of concomitant medication within its therapeutic level by the unblinded investigator. In this way the blinded set-up of the trial will also be respected. Another possibility in avoiding pro. blems with changing plasmalevels of concomitant antiepileptic drugs is in advance of a placebo-controlled study performing of an open label loading trial to maximum tolerated dose or significant reduction in seizure frequency. By dropping-out those patients who need change in concomitant medication, the remainders will not need correction of their antiepileptic drugs during the trial. Although this design is atractive, there is the risk of sellection bias. The remaining group of patients can be genetically determined, e.g. slow metabolizers of phenytoin (Ferrari et al. 1990).

The double-blind, placebo-controlled study was designed as a parallel study. Crossover studies are much more efficient compared to parallel studies; parallel studies generate to same statistical power by including between 2.7 to 6.4 as many patients as in crossover (Scoville et al. 1981). However, in the case of loreclezole this design will be unpractical. With a half-life of about 20 days the washout period will result in an unacceptable long course of the trial.

Responders to an investigational drug mostly want to continue on the drug, even if it is not yet marketed. For ethical reasons this must be allowed. Its is reasonable to design follow-up trials for all patients treated with investigational antiepileptic drugs like loreclezole. Primarily for safety reasons repeating control of clinical and laboratory parameters must be perfomed. Another reason for follow-up protocols is investigation of less easy to analyse effects, e.g. on performance, tolerance to the beneficial effects, or toxicity. At random continuation of the treatment erase the possibility of evaluating important effects on parameters. For better long term compliance to investigations, trial designs must be as easy and unburdening as possible.

\subsection{Conclusions}

In the studies reported in this thesis, loreclezole has proved to be a safe and usefuldrug against previously pharmacotherapy-resistant epilepsy. It is effective against partial seizures, and probably also against generalized seizures. Loreclezole is tolerated well by most patients and seldom leads to side effects if the plasma concentration does not exeeed $10 \mathrm{mg} / \mathrm{l}$. Investigation of loreclezole monotherapy has been restricted by the 
unsuitability of the patient population for such treatment. Nevertheless, in the patients who were finally adjusted to loreclezole monotherapy the therapeutio outcone was good. Interaction with existing comedication seems unimportant for valproate, of minor importance for carbamazepine and of some importance for phenytoin. In some patients using loreclezole the plasma phenytoin concentration rose higher than during placebo treatment. Reduction of the phenytoin dose is indicated if intoxication symptoms appear. The desmethylclobazam/clobazam ratio becomes higher after introduction of loreclezole. Although this finding is of chemical interest, its clinical impact is probably negligible.

At a plasma concentration of $2-5 \mathrm{mg} / 1$ the seizure frequency is reduced by $50 \%$ or more in about $30 \%$ of the patients. This result can become even better at higher dostges. Loreclezole is generally well tolerated. As yet, the weight loss that occurs in some patients is inexplicable. In view of the zero order kinetics above 1 - $2 \mathrm{mg} / \mathrm{l}$. plasma concentration, the dosing of loreclezole requires regular laboratory determinations of the plasma concentration in addition to evaluation of the clinical picture.

In the studies described in this thesis the mean loreclezole dose was $75-125 \mathrm{mg} / \mathrm{day}$, given once per day. The long plasma half-life certainly has advantages, but also has disadvantages such as the slow disappearance of side effects (somnolence and ataxia) at excessive doses. In the studies reported here there were no important changes in chemical or haematological findings. At a dosage giving plasma concentrations of $5-10 \mathrm{mg} / \mathrm{l}$, loreclezole is evidently an effective antiepileptic drug and represents an advance in the treatment of epilepsy. Results available from a limited number of patients show that loreclezole is also effective as monotherapy. Animal experiments show that the efficacy of loreclezole probably extends beyond partial seizures to other types of seizure too. How broad the indication for loreclezole is will become clear from further investigation. In view of the profile of efficacy and safety, a positive role in the treatment of patients with epilepsy is to be expected. 


\section{SUMMARY}

In Chapter 1 a description of the problems posed by pharmacotherapy-resistant epilepsy is followed by a survey of commonlly used antiepileptic drugs. A group of drugs previously unused in epilepsy treatment, the imidazoles and triazoles, is then introduced and various animal experiments with R 57720 and loreclezole (R 72063) are mentioned. Problems posed by clinical trials are discussed: the selection of patients, the evaluation of the results, some ethical aspects, and the hurdles that a new substance has to clear before being recognized as an effective drug.

Chapter 2: After its safety and efficacy had been demonstrated in animal studies, $R$ 57720 was administered to volunteers. The influence of a single dose on comedication and on sensitivity to light flashes was then investigated in patients. In a 4-week study with a low add-on dose in antiepileptic drug users there was no detectable influence on biochemical or haematological parameters. The substance was tolerated well. In an open add-on treatment $R 57720$ was given to 11 sufferers from partial epilepsy at dosages rising from 120 to $240 \mathrm{mg} / \mathrm{day}$, the need for a dosage increment being decided on the basis of efficacy criteria. One patient developed what was presumed to be an allergic reaction to the substance and in 1 patient the concentration of the comedication rose to toxic levels. In 6 patients the seizure frequency was reduced by more than $50 \%$. In the weeks following the trial, 3 patients whose $\mathrm{R} 57720$ treatment was continuing developed hepatotoxic symptoms, which vanished rapidly after withdrawal of $R 57720$. Two patients used R 57720 for more than 2 years with good therapeutic effect and no side effects. In wiew of the hepatotoxic symptoms, R 57720 was not developed or investigated further.

In Chapter 313 patients suffering from pharmacotherapy-resistant epilepsy were treated with loreclezole at a dosage giving a plasma concentration of $1-3 \mathrm{mg} / \mathrm{l}$. There were no side effects attributable to the test substance. Seizure frequency fell by $30 \%$ on average, and by more than $50 \%$ in $40 \%$ of the patients. Two patients obtained an unfavourable effect, but none of the patients experienced severe adverse events. Two patients had a considerable reduction in severity of seizures without any reduction in frequency. Eleven patients decided to continue using loreclezole after the end of this study.

Patients reeruited to the study described in Chapter 4 on the basis of either an undeniable reduction in seizure frequency or a reduction in the severity of the seizures were treated for a further year with loreclezole, the comedication remaining - in principle unchanged. All the patients tolerated loreclezole well throughout this year; no serious side effects were reported. In some patients the loreclezole dosage was raised on the basis of clinical effect and plasma concentration. Mean seizure control was maintained; relative to the baseline value the mean seizure reduction was about $40 \%$, relative to the study reported in Chapter 3 there was a negligible increase of $3 \%$ in seizure frequency. Four of the 1 I patients obtained a seizure reduction of $50 \%$ or more relative to baseline. 
To provide an objective assessment of the efficacy and safety of loreclezole; in Chapter $\mathbf{5}$ a double-blind placebo-controlled parallel study was designed. The study was a multicentre one. Sixty-two pharmacotherapy-resistant patients with 4 or more seizures per month were evaluable. The patients were matched in pairs according to seizure frequency. Nevertheless, the active treatment group had a somewhat higher seizure frequency than the placebo group. Although both increases and decreases in seizures were seen in each group, in the placebo group the maximum seizure reduction was $42 \%$ and this $42 \%$ reduction was only seen in 1 patient, whereas in the active treatment group 6 of the 32 original patients had a seizure reduction of $50 \%$ or more. The maximum reduction in seizure frequency in this group was $76 \%$. There were no serious adverse events or significant interactions with valproate, carbamazepine or phenytoin. In 3 patients in the active treatment group the comedication (carbamazepine and phenytoin) was rediced somewhat on account of intoxication symptoms. Although there was interaction with clobazam, notably a reduction in desmethylclobazam/clobazam ratio in the active treatment group, this interaction was of no consequence for the treatment. Haematological and biochemical parameters were not adversely affected.

At the end of the double-blind placebo-controlled study of the efficacy and safety of loreclezole reported in Chapter 5, patients who had participated in that study were given the opportunity (if they had been taking placebo) to begin loreclezole treatment or (if they had been taking loreclezole) to continue the loreclezole treatment, at a higher dosage if appropriate (Chapter 6). The study aim was to evaluate the efficacy and safety of loreclezole in long term treatment and with more patients. The inclusion and exclusion criteria were the same as for the double-blind placebo-controlled study, except for seizure frequency. Existing antiepileptic therapy was retained unchanged during the treatment. Loreclezole was dosed to a plasma concentration of about $5-7 \mathrm{mg} / \mathrm{l}$, depending on the results of the treatment. Loreclezole was effective in reducing the seizure frequency in 22 of the 56 patients (a seizure reduction of more than $50 \%$ with respect to baseline), whilst there were another 17 patients who experienced some reduction in their seizure frequency (a reduction of $25-50 \%$ ). In this study too the severity of the seizures was not considered in evaluating the effect. There were no adverse events requiring modification of the treatment. There were no important interactions with the existing antiepileptic drugs. The conclusion from this study was that loreclezole is a safe and effective drug in the treatment of serious epilepsy.

Chapter 7: In the pharmacotherapy of epilepsy, monotherapy is preferable among others on account of better compliance, reduced side effects, absence of drug-drug interactions. An antiepileptic drug has true value only if it is effective as monotherapy. Accordingly, patients who had been treated with loreclezole as add-on therapy in previous studies were invited to take part in a study in which the comedication would be gradually withdrawn. A few patients who had not previously taken loreclezole were also treated in accordance with the same protocol. Under the control of clinical monitoring and clinicochemical investigations, the comedication was reduced in accordance with a dosage reduction scheme. In 7 out of 9 patients the therapeutic effect of loreclezole was adequately maintained during the monotherapy that followed the withdrawal of comedication. It can be concluded that loreclezole is not just useful as add-on medication; in monotherapy too it is well tolerated and has a good therapeutic effect. 
The final Chapter 8 gives an overview of the research reported in this thesis and its clinical importance for the treatment of epilepsy patients. Loreclezole is effective in the treatment of patients with serious epilepsy. Although the studies comprised almost exclusively patients with partial seizures, animal experiments show that loreclezole is likely to be a brond-spectrum antieplleptic drug. Further research is therelore desirable. For partial seizures the reduction in seizures is about $30 \%$ if all patients are laken together. None of the patients showed serious deterioration. In the patients who obtained improvement from the loreclezole treatment no tolerance of the therapeutic effect of loreclezole developed, even after more than a year of treatment. The minimum effective loreclezole dose correlated with a plasma concentration of more than $2 \mathrm{mg} / \mathrm{l}$. The greatest chance of successful treatment, however, is to be expected at plasma concentrations between 5 and $10 \mathrm{mg} / 1$. The dosage necessary to reach such concentrations was $1 \mathrm{mg} / \mathrm{kg}$ body weightday on average, but showed strong interindividual variations. The loreclezole dose should be adjusted individually in accordance with the clinical picture; plasma concentration determinations may be helpful. Plasma concentrations above $10 \mathrm{mg} / \mathrm{l}$ generally lead to side effects in the form of somnolence and ataxia. These symptoms were fully reversible on reduction of the dose. The weight loss observed in some loreclezole users has not been explained.

Owing to the long plasma half-life a certain time must elapse before the effect of a change of dose can be evaluated. On the other hand it is an advantage that loreclezole does not need to be taken frequently. For most patients one dose per day is convenient. In the event of an acute problem that prevents oral administration, the plasma concentration remains at a therapeutic level for a period that is sufficiently long to obviate the need for a parenteral dosage form to bridge the timegap. When loreclezole was withdrawn on account of inefficacy, 10 pacients stopped the medication abruptly without experiencing withdrawal seizures.

Loreclezole seems to be a valuable supplement to the arsenal of antiepileptic drugs. 


\section{SAMENVATTING}

Na de problemen rond farmacotherapie resistente epilepsie patiènten beschreven te hebben, volgt in hoofdstuk 1 een overzicht van de thans gebruikelijke anti-epileptica. Vervolgens wordt aandacht besteed aan een niet eerder in de epilepsie bestrijding gebruikte groep middelen, te weten de imidazolen en triazolen en aan diverse dierexperimenten met R 57720 en loreclezole. In het laatste deel van het hoofdstuk worden problemen trond patiënten onderzoek belicht. Achtereenvolgens komen aan de orde de selectie van patiënten, het beoordelen van de resultaten, enkele medisch-ethische aspecten alsmede de weg die een nieuw geneesmiddel heeft af te leggen voor erkenning als effectief medicijn.

Hoofdstuk 2: R 57720 werd na gebleken veiligheid en effectiviteit in dierproeven toegediend aan vrijwilligers. Vervolgens werd in eenmalige dosering bij patiënten onderzoek verricht naar invloed op comedicatie en lichtflitsgevoeligheid. In een vier weken durende proef met een lage dosis R57720, toegediend aan gebruikers van anti-epileptica (add-on) kon geen invloed gevonden worden op biochemische en hematologische parameters.De stof werd goed verdragen. In een open add-on behandeling werd $R 57720$ aan elf lijders aan partiële epilepsie toegediend in een oplopende dosering van 120 to 240 mg per dag. Daarvoor was er een beslisschema aan de hand van de effectiviteitscriteria. Een patiënt ontwikkelde een vermoedelijk allergische reactie voor de stof en bij een patiënt steeg de comedicatie tot toxische waarden. Bij zes patiënten werd een aanvalsreductie van meer dan vijftig procent gemeten. Drie patiënten ontwikkelden in de weken na de trial bij continueren van $\mathrm{R} 57720$ hepatotoxische verschijnselen, die na staken van $R 57720$ snel verdwenen. Twee patiënten hebben met goed therapeutisch effect en zonder bijwerkingen ruim twee jaar R 57720 gebruikt. De hepatotoxische verschijnselen waren reden R 57720 niet verder te ontwikkelen of te onderzoeken.

In hoofdstuk 3 werden dertien patiënten lijdende aan farmacotherapie resistente epilepsie behandeld met loreclezole. Gestreefd werd naar een plasmaconcentratie van 1 tot 3 $\mathrm{mg} / \mathrm{l}$. Er werden geen bijwerkingen geconstateerd die toe te schrijven waren aan de onderzochte stof. De aanvalsfrequentie daalde gemiddeld $30 \%$, bij $40 \%$ van de patiën. ten meer dan $50 \%$. Bij twee patiënten werd een ongunstig effect verkregen, doch geen van de behandelden ervoer ernstige bijwerkingen. Twee patiènten hadden cen aanzienlijke vermindering van aanvalsernst, zonder een frequentiereduktie. Elf patiënten besloten na beëindiging van deze studie door te gaan met het gebruik van loreclezole.

In hoofdstuk 4 werden patiënten die daarvoor in aanmerking kwamen, hetzij door een ontegenzeglijke vermindering van de aanvalsfrequentie, dan wel door een vermindering van de ernst van de aanvallen gedurende een jaar verder behandeld met loreclezole, waarbij de comedicatie in principe niet veranderd werd. Alle patiënten hebben ook gedurende dit jaar loreclezole goed verdragen, emstige bijwerkingen werden niet gemeld. Bij enkele patiënten werd de dosering loreclezole verhoogd op geleide van klinisch effect en plasma concentratie. De gemiddelde aanvalscontrole bleef gehandhaafd, ten opzichte van de uitgangswaarde was de gemiddelde aanvalsreductie circa $40 \%$, ten opzichte van de studie genoemd in hoofdstuk 3 was er een verwaarloosbare toename van de aanvalsfrequentie van $3 \%$. Vier van de elf patiënten hield een aanvalsreductie van $50 \%$ of meer ten opzichte van de uitgangswaarde. 
Hoofdstuk 5: Om de effectiviteit en veiligheid van loreclezole te objectiveren werd een dubbelblind placebo gecontroleerd onderzoek opgezet. Het onderzoek werd multicentrisch verricht: Tweeénzestig farmacotherapie resistente patiënten met vier of meer aanvallen per maand waren voor het onderzoek evalueerbaar. De patiènten werden in paren gematched naar aanvalsfrequentie. Dit kon echter niet verhinderen dat uiteindelijk de verumgroep een iets hogere aanvalsfrequentie bleek te hebben dan de placebogroep. In beide groepen waren zowel toename als afname van anvallen waar te nemen. In de placebogroep was echter de maximale aanvalsreductie $42 \%$ bij slechts een patiënt, in de verumgroep waren er zes van de 32 aanvankelijke patiënten die een aanvalsreductie hadden wan $50 \%$ of meer. De maximale aanvalsreductie was $76 \%$. Enstige bijwerkingen of significante interactie met valproaat, carbamazepine en phenytoine werden niet gemeld. Bij drie patiènten in de verum groep werd de comedicatie (carbamazepine en phenytoine) wegens intoxicatieklachten enigszins verminderd. Er was wel interactie met clobazam, met name de ratio desmethylclobazam/clobazam verminderde in de verum groep, maar consequenties voor de behandeling had dat niet. Haematologische en biochemische parameters werden niet nadelig beïnvloed.

Patiënten die meegedaan hadden aan de dubbelblind, placebo gecontroleerde studie naar de effectiviteit en veiligheid van loreclezole, konden na beëindiging van de studie genoemd in hoofdstuk 5 beginnen met het gebruik van loreclezole, indien zij behandeld waren met placebo of doorgaan met het gebruik van loreclezole, eventueel in een hogere dosering (hoofdstuk 6). De studie was erop gericht op langere termijn en bij meer patiënten de effectiviteit en veiligheid van loreclezole te evalueren. In- en uitsluitingscriteria waren gelijk aan die voor de dubbelblind uitgevoerde, placebo-gecontroleerde studie, met uizondering van het bepaalde ten aanzien van de aanvalsfrequentie. Tijdens de behandeling werd de bestaande anti-epileptische therapie ongewijzigd gelaten. Loreclezole werd afhankelijk van de behandelingsresultaten gedoseerd tot een plasmaconcentratie van ongeveer 5 tot $7 \mathrm{mg} / \mathrm{l}$. Loreclezole was effectief in het reduceren van de aanwalsfrequentie bij 22 van de 56 patiënten (een aanvalsreductie van meer dan $50 \%$ vergeleken met de uitgangswaarde), terwijl er 17 patiënten waren die enige reductie van hun aanvalsfrequentie ervoeren ( 25 tot $50 \%$ minder). Ook in dit onderzoek werd niet getet op verandering in de ernst van de aanvallen bij het beoordelen van het effect. Bijwerkingen die noopten tot ingrijpen in de behandeling werden niet gemeld of geconstateerd. Belangrijke interacties met de bestaande anti-epileptica werden niet gevonden. De conclusie uit dit onderzoek is dat loreclezole een veilig en effectief middel is bij de behandeling van ernstige epilepsie.

Hoofdstuk 7: Bij de farmacotherapeutische behandeling van epilepsie geniet monotherapie de voorkeur ${ }_{*}$ onder andere wegens belere therapietrouw en vermindering van bijwerkingen. Een anti-epilepticum heeft pas dan werkelijk waarde als het in monotherapie effectief is. Dat was de reden in aansluiting op de gedane studies patiënten, die behandeld waren met loreclezole als add-on therapie, uit te nodigen voor een studie waarin de comedicatie geleidelijk gestaakt werd. Tevens werden enkele patiënten, die niet eerder loreclezole hadden gebruikt, volgens hetzelfde protocol behandeld. Volgens een schema werd de comedicatie onder controle van klinische verschijnselen en klinisch-chemisch onderzoek gereduceerd. Het therapeutisch effect van loreclezole bleek in monotherapie, bereikt na onttrekken van comedicatie, bij zeven van negen patiënten voldoende 
gehandhaafd. Hieruit kan geconcludeerd worden dat loreclezole niet alleen als add-on medicatie nuttig is; ook in monotherapie wordt het goed verdragen met goed therapeutisch effect.

In het afsluitende hoofdstuk 8 werd een overzicht gegeven van het in dit proefschrift verrichte onderzoek voor zover het van klinisch belang is in de behandeling van patiënten met epilepsie. Loreclezole blijkt effectief bij de behandeling wan patiënten met ernstige epilepsie. In de onderzoeken zijn weliswaar vrijwel uitsluitend patiènten behandeld met partiële aanvallen, maar op grond van dierexperimenteel onderzoek mag verwacht worden dat loreclezole een breed spectrum anti-epilepticum zal blijken. Verder onderzoek is daarom gewenst. Bij partiële aanvallen lijkt circa $30 \%$ aanvalsreductie op te treden als alle patiënten tezamen worden genomen. Enstige verslechtering trad bij geen van de patiënten op. Bij de patiënten die baat hadden bij behandeling met loreclezole trad ook na meer dan een jaar behandelen geen tolerantie van het therapeutische effect van loreclezole op. De minimale effectieve dosis loreclezole correleerde met een plasmaconcentratie van meer dan $2 \mathrm{mg} / \mathrm{l}$. De grootste kans op succes van de behandeling werd echter verkregen bij plasma concentraties tussen de 5 en $10 \mathrm{mg} / \mathrm{l}$. Deze waarde werd bereikt bij een dosering van gemiddeld $1 \mathrm{mg} / \mathrm{kg}$ lichaamsgewicht/dag, maar kan door sterk individueel bepaalde omstandigheden hiervan afwijken. De dosis loreclezole dient individueel aangepast te worden op geleide van het klinische beeld, waarbij plasmaconcentratie bepalingen behulpzaam kunnen zijn. Bij plasmaconcentraties boven $10 \mathrm{mg} / \mathrm{l}$ traden in de regel bijwerkingen op in de zin van slaperigheid en ataxie. Deze klachten waren volledig reversibel na vermindering van de dosis. Niet verklaard is het gewichtsverlies dat bij een aantal gebruikers van loreclezole optrad. Door de lange plasmahalfwaarde tijd is het effect van dosis verandering pas na geruime tijd te evalueren. Anderzijds is het voordeel dat loreclezole weinig frequent ingenomen hoeft te worden. Voor de meeste patiënten is eenmaal daags innemen van de medicatie gemakkelijk. Bij (acute) problematiek die oraal innemen onmogelijk maakt, blijft de plasmaconcentratie dermate lang op therapeutisch niveau dat een parenterale toedieningsvorm niet noodzakelijk is om problemen in deze te bestrijden. Bij staken van loreclezole wegens ineffectiviteit werd bij tien patiënten van de ene op de andere dag de medicatie gestaakt, zonder optreden van onttrekkingsinsulten.

Loreclezole lijkt een waardevolle aanvulling op het arsenaal van de medicamenteuze behandeling van epilepsie. 


\section{REFERENCES}

- American Academy of Nearology (the Therapeutic and Technology Assessment Subcommittee Assessment: generic substitution for antiepileptic medication. Neurology 1990; 40:1541-1643 - Andersen G, Pedersen B. Valproate monotherapy in elderly patients with epilepsy. Epilepsia $1989,30,663$

- Anderson GD. Phenobarbital - Biotransformation. In: Levy RH, Dreifuss FE, Mattson RH, Meldrum BS, Penry JK, eds. Antiepileptic drugs. New York: Raven, 1989:305.312

- Arends J, Declerck AC, Janssen F, De Beukelaar F. R 72063 . Het effect van $50 \mathrm{mg}$ en $100 \mathrm{mg}$ op EEO-activiteit. Janssen Research Proklucts Information Service, report $R$ 72 063/67-NL, Tilburg: mart 1988

- Arezzo JC, Schroeder CE, Litwak MS, Steward DL. Effects of vigabatrin on evoked potentials in dogs. Br J Clin Pharmac 1989; 27 (suppl 1):53S-60S

- Arnoldussen WJ. Trileptal (oxcarbazepine, OCB) versus Tegretol (CBZ) - Alternative methods 10 meastre seizure control. 18 th International Epilepsy Congress, Dethi, 1989

- Baille TA, Rettenmeier AW. Valproate - Biotransformation. In: Levy RH, Dreifuss FE, Mattson RH, Meldrum BS, Perry JK, eds. Antiepileptic drugs. New York: Raven, 1989:601-619

- Baker GA, Smith DF, Dewey M, Morrow J, Crawford PM, Chadwick DW. The development of a seizure severity scale as an outcome measure in epilepsy. Epilepsy Res $1991 ; 8: 245-251$

- Bancaud J. Kojewnikov"s syndrome (epilepsia partialis continua) in Children. In: Roger $J$, Draver $C$, Bureau $M$, Dreifuss FE, Wolf $P$, eds. Epilleptic syndromes in infancy, childhood and arlolescence. London: John Libbey Eurotext, 1985:286-298

- Banes DM, Dichter MA. Effects of ethosuximide and tetramethylsuccimide on cultured cortical neurons. Neurology 1984; 34:620-624

- Baruzzi A, Michelucci R, Tassinari CA. Benzodiazepines - Nitrazepam. In: Levy RH, Dreifuss FE, Mattson RH, Meldrum BS, Penry JK, eds. Antiepileptic drugs. New York: Raven, 1989:785804

- Beaumont D, Chiron C, Palacios L, Luna D. Dulac O, Murnford J. Vigabatrin in the management of drug resistant infantile spasms. Acta Neurol Scand 1990; 82 (suppl 133):34 - Bener LZ, Mitchell JR, Sheiner LB. Pharmacokinetics: the dynamics of drug absorption, distribution, and elimination. In: Goodman Gilman A. Rall TW, Nies AS, Taylor P. Goodman and Gilman"s the pharmacological basis of therapeutics. New York: Perganon, 1990:3-32

- Benet L.Z. Principles of prescription order writing and patient compliance instructions. In: Goodman Gilman A, Rall TW, Nies AS, Taylor P. Goodman and Gilman's the pharmacological basis of therapeatics. New York: Pergamon, 1990:1640-1649

- Ben-Menachem E. Vigabatrin in epilepsy - an irreversible GABA-transaminase inhibitor. Goteborg: University of Göteborg. 1989

- Betger Y. Loisea P. Depakine Chrono 500, comprimés pelliculés a libération prolongée; rapport d"expert sur la documentation clinique. Montpelier: Sanofi 491.6004 , part I C 3, 1986 - Binnie CD. The use of the inter-ictal EEG in the study of antiepileptic drugs. In: Buser PA, Cobb WA, Okuma T, eds Kyoto Symposia. Electroenceph Clin Nenrophysiol 1982; (suppl 36): $504-512$

- Binnie CD. De Beukelar F, Meijer JWA, Overweg J, Vanden Bussche G. Pilot single dose interaction- and efficacy study of R 57720 administered as "add-on" therapy in therapy resistant epileptic patients. Janssen Research Produci Information Service, Clinical Research Report, R 57720/. Beerse March 1985 
Binnie CD. Progress in the treatment of epilepsy. J Neurol Neurosurg Psychiatry 1990:53:273274

- Bolton JB, Rimmer E, Williams $J$, Richens A. The effect of vigabatrin on brain and platelet GABA-transaminase activities. Br J Clin Pharmac 1989; 27 (suppl 1):35S-42S

- Bolwig TG. Do kindling-like phenomena unify hypothesis of psychopathology? In: Bolwig TG, Trimble MR, eds. The clinical relevance of kindling. Chichester: Wiley, 1989;1-13

- Bossi L, Battino D, Caccamo ML, Canger R, Como ML, De Giambetista M, Marini MA. Pardi $G$, Pifarotti $G$, Porro MG, Rovei V, Sanjuan P, Sereni F. Klinische und pharmakologische Beobachtungen bei 31 Neugeborenen won epileptischen Müttern. In: Doose H, Dam M, GrokSelbeck G, Meinardi H, eds. Epilepsie 1979, Ehe, Schwangerschaft, Geburt, genetische Beratung. Stuttgart: Thieme, 1980:23-27

- Bourgeois B, Beaumanoir A, Blajev B, De la Cruz N, Despland PA, Egli M, Geudelin B, Kaspar U, Ketz E, Kronauer $\mathrm{Ch}$, Meyer Ch, Scollo-Lavizzari G, Tosi C, Vassella F, Zagury $S$. Monotherapy with valproate in primary generalized epilepsies. Epillepsia 1987; 28 (suppl 2):S8-S11 - Bourgeois BFD. Valproate - Clinical use. In: Levy RH, Dreifuss FE, Mattson RH, Meldrum BS, Penry JK, eds. Antiepileptic drugs. New York: Raven, 1989:633-642

- Bowyer JF. Phencyclidine inhibition of the rate of development of amygdaloid kindled seizunes. Exp Neurol 1982; 75:173-183

- Breimer DD. Drug-drug interactions: summary of general principles. In: Breimer DD, Merkus FWHM, eds. Drug-drug interactions and food-drug interactions. Boerhave Commissie voor Postacademisch Onderwijs in de Geneeskunde, Boerhaave cursus. Leiden: Rijksuniversiteit Leiden 1991:1-4

- Brodie MJ. Established anticonvulsants and treatment of refractory epilepsy. Lancet 1990; $336: 350-354$

- Browne TR, Feldman RG. Clinical experience with benzodiazepines in neurological disorders. In: Priest RG, Vianna Filho U. Amrein R, Skreta M, eds. Benzodiazepines, today and tomorrow. Lancaster: MTP, 1980:113-122

- Browne TR, Chang T. Phenytoin - Biotransformation. In: Levy RH, Dreifuss FE, Mattson RH, Meldrum BS, Penry JK, eds. Antiepileptic drugs. New York: Raven, 1989:197-213

- Browne TR, Mattson RH. Penry JK, Smith DB, Treiman DM, Wilder BJ, Ben-Menachem E, Miketa RM, Sherry KM, Szabo GK. A multicentre study of vigabatrin for drug-tesistant epilepsy. Br J Clin Pharmac 1989;27 (suppl 1):95S-100S

- Brunia CHM, Arends J, De Beukelaar F. Smakman J, Tritsmans L. Tolerability study of ascending plasma levels of loreclezole (R72063) in volunteers. Part I: Safety evaluation. Janssen Research Product Information Service, Clinical Research Report, R 72063/13 (N 71375), Beerse: December 1989

- Buchanan RA, Kinkel AW, Smith TC. The absorption and excretion of ethosuximide. Int I Clin Pharmacol 1973:7:213-218

- Buchthal F, Svensmark O, Schiller PJ. Clinical and electroencephalographic correlations with serum levels of diphenylhydantoin. Arch Neurol $1960 ; 2: 624-630$

- Burton BS. On the propyl derivates and decomposition products of ethyl acetoacetate. Am Chem J 1882; $3.385-395$

- Callaghan DA, Schwark WS. Pharmacological modification of amygdaloid.kindled seizures. Neuropharmacology 1980; 19:1131-1136

- Callaghan N, Goggin T. Adjunctive therapy in resistant epilepsy. Epilepsia 1988; 29 (suppl 1): $\$ 29-\$ 35$ 
- Canadian Clobazan Cooperative Group. Clobazam in treatment of refractory Epilepsy: the Canadian experience. A retrospective study. Epilepsia 1991:32:407-416

- Camnon DJ, Mumford JP, Butler WH. Neuropathological investigations in vigabatrin treated patients. Acta Newrol Scand 1990; 82 (suppl 133):38

- Cenrud B, Guyou M, Levy RH, Brachet-Liemain, Morselli PL. Loiseau P. Effect of dosage regimen on walproic acid plasma levels. In: Dam M, Gram L, Perry JK, eds. Advances in epileptology: XIIth Epilepsy International Symposium. New York: Raven, 1981:549-553

- Chadwick DW. Valproate monotherapy in the management of generalized and partial seizures. Epilepsia, 1987: 28 (suppl 2):\$12-\$17

- Chadwick D. Diagnosis of epilepsy. In: Epilepsy, a Lancet Review 1990a:15-18

- Chadwick D. Prospects for new drug treatment in epillepsy: a review. I R Soc Med 1990b; $83: 383-386$

- Chang T. Ethosuximide - Absorption, distribution, and exeretion. 1n: Levy RH, Dreifuss FE, Mattson RH, Meldrum BS, Penry JK, eds. Antiepileptic drugs. New York: Raven, 1989a:671-678 - Chang T. Ethosuximide - Biotransformation. In: Levy RH, Dreifuss FE, Mattson RH, Meldrum BS, Penry JK, eds. Antiepileptic drugs. New York: Raven, 1989b:679-683

- Chepman AG, Graharn JL. Patel S, Meldrum BS. Anticonvulsant activity of two orally active competitive $N$ methyl-D-aspartate anatagonists, CGP 37849 and CGP 39551, against soundinduced seizures in DBA/2 mice and photically induced myoclonus in Papio papia. Epilepsia $1991 ; 32: 578-587$

- Cobos JE. High-dose phenytoin in the treatment of refractory epilepsy. Epilepsia 1987; 28:1111.14

- Commission on Antiepileptic Drugs of the International League Against Epilepsy. Guidelines for clinical evaluation of antiepileptic drugs. Epilepsia 1989; 30:400-408

- Commission on Antiepileptic Drugs of International League Against Epilepsy. Workshop on antiepileptic drug trials in children. Epilepsia $1991 ; 32: 284-285$

- Commission on Classification and Terminology of the International League Against Epilepsy. Proposal for revised clinical and electroencephalographic classification of epileptic seizures. Epilepsia 1981; 22:489-501

- Conmission on Classification and Terminology of the International League Against Epilepsy. Proposal for revised classification of epilepsies and epileptic syndromes. Epilepsia 1989:30:389399

- Cosi V. Callieco R, Galimberti CA, Manni R., Tartara A, Mumford J, Penucca E. Effects of vigabatrin on evoked potentials in epileptic patients. Br J Clin Pharmac 1989; 27 (suppl 1): 61S$68 \mathrm{~S}$

- Costa E. Corda MG Epstein B. Forchett C, Guidotti A. GABA-benzodiazepine interactions. In: Costa E, ed. The benzodiazepines: from molecular biology to clinical practise. New York: Raven Press, 1983:1117-136

- Covanis A, Jeavons PM. Gupta AK. Monotherapy with once daily sodium valproate (Epilim). In: Dam M, Gram L, Penry JK, eds. Advances in epileptology: XIIth Epilepsy International Symposium. New York: Raven, 1981:527-532

- Cramer JA, Smith DB, Mattson RH, Delgado Escueta AV, Collins JF, and the VA Epilepsy Cooperative Study Group. A method of qualification for the evaluation of antiepileptic drug therapy. Neurology 1983; 33 (suppl 1):26-37

- Czernik AJ, Petrack B. Kalinsky HJ, Psychoyos S, Cash WD. Tsai C, Rinehart RK, Granat FR. Lovell RA. Brundish DE. Wade R. CGS 8216 : receptor binding characteristics of a potent benzodiazepine antagonist. Life Sci 1982; 30:363-372 
- Dam M. Philbert A. Drug treatment of pregnant epileptic patients. In: Shorvon $S$, Birdwood GFB, eds. Rational approaches to anticonvulsant drug therapy. Bern: Hans Huber Publishers, $1984: 55-58$

- Dean JC, Penry JK. Valproate monotherapy in 30 patients with partial seizures. Epilepsia 1988; 29:140-144

- Dean $\mathbb{~} C$, Penry JK General principles - Discontinuation of antiepileptic drugs. In: Levy RH, Dreifuss FE, Mattson RH, Meldrum BS, Penry JK, eds. Antiepileptic drugs. New York: Raven, 1989:133-142

- De Beukelaar F, Declerck AC, Hulsman JARJ, Janssen AMAI, Nujjten STM, Vanden Bussche G, Van der Ham-Veltman PHM. R 57720 - $40 \mathrm{mg}$ as add-on therapy in therapy resistant epileptic patients. Analysis of the salety data. Janssen Research Product Information Service, Clinical Research Report, R 57720/5, Beerse: November 1984

- De Deyn PP, Marescau B, Macdonald RL. Epillepsy and the GABA-hypothesis a brief review and some examples. Acta Neurol Belg. 1990; 90:65-81

- De Giorgio CM, Rabinowicz AL, Olivas RD. Carbamazepine-induced antinuclear antibodies and systemic lupus erythematosus-like symdrome. Epilepsia 1991: 32:128-129

- Dehling E, Giuccioli D, Leitenberger J, Stich K, Wolf P. Treatment with bromide in adult patients with epilepsy: a retrospective study. Epilepsia 1990;31:226

- DeLorenzo RJ. Mechanisms of action of anticonvulsant drugs. Epilepsia 1988; 29 (suppl 2):\$35 $\$ 47$

- DeLorenzo RJ. Phenytoin - Mechanisms of action. In: Levy RH, Dreifuss FE, Mattson RH, Meldrum BS, Penry JK, eds. Antiepileptic drugs. New York: Raven, 1989:143-158

- DePew C, Lubozynski M. Efficacy, toxicity and compliance in the reduction of antiepileptic drugs from polytherapy to monotherapy. Epilepsia 1989;30:680

- Dhalla Z, Bruni J, Sutton J. A comparison of the efficacy and tolerability of controlled-release carbamazepine with conventional carbamazepine. Can J Neurol Sci 1991; 18:66-68

-Dhuna A, Pascual-Leone A, Talwar D. Exacerbation of partial seizures and onset of nonepileptic myoclonus with carbamazepine. Epilepsia 1991; 32:275-278

- Dichter MA. Cellular mechanisms of epillepsy and potential new treatment strategies. Epilepsia 1989; 30 (suppl 1):S3-SI2

- Doose H. Zerebrale Anfälle im Kindesalter. Flensburg: Severin, 1983

- Dravet C, Legra C, Julian C, Aymé S, Trentin G, Magaudda A. Teratogenity of anticonvulsant drugs: results of the southern of France prospective study. 18th International Epilepsy Congress. Dellhi, 1989

- Dreifuss FE Foreword - Gamma vinyl GABA (vigabatrin) in epilepsy: the international experience. Epilepsia 1989a; 30 (suppl 3); wii

- Dreifuss FE. Ethosuximide - Toxicity. In: Levy RH, Dreifuss FE Mattson RH, Meldrum BS, Penry JK, eds. Antiepileptic drugs. New York: Raven, 19896:699-705

- Dreifuss FE. Valproate - Toxicity. In: Lewy RH, Dreifuss FE, Mattson RH, Meldrum BS, Penry JK, eds. Antiepileptic drugs. New York: Raven, 1989bc:643-651

- Dreifuss FE. Hepatic side effects of valproate. In Chadwick D, ed. Fourth International Symposium on sodiun walproate. London: Royal Society of Medicine, 1990:150 153

- Duncan JS. Sander JWAS. The Chalfont Seizure Severity Scale. Acta Neurol Scand 1990; 82 (suppl 133):31

- Duncan JS, Shorvon SD, Trimble MR. Discontimuation of phenytoin, carbamazepine, and walproate in patients with active epilepsy. Epilepsia 1990; 31:324-333 
- Duncan IS. Modern treatment strategies for patients with epilepsy; a review. J R Soc Med 1991; $84159 \times 162$

- Duncan JS, Patsalos PN, Shorvon SD. Effects of discontinuation of phenytoin, carbamazepine. and walproate on concomitant antieplleptic medication. Epilepsia 1991; 32:101-115

- Dupuis HM: Infourmed consent; verdwazing van onze tijd? In: Engberts DP. Bennebroek Graventionst I. Dupuis HM, Vermey $P$, Wintzen $A R$, eds. Informed consent: schijn en werkelijkheid. Boerhave Conmissie voor Postacademisch Onderwijs in de Geneeskunde, Boethanwe cursus. Leiden: Rijksuniversiteit Leiden 1988:81-87

- Earl Walker A. Surgery for epliepsy. In: Magnus O, Lorentz de Haas AM, eds. The epilepsies. NorthHolland Publishing Company Amsterdam 1977:491-497 (Vinken PJ, Bruyn GW, Handbook of clinical nearology, wol 15 )

- Eadie MJ. Pharmacokinetics of the anticonvulsant drugs. In: Tyrer JH, ed. The treatment of epilepsy. Lancaster: MTP, 1980a:95-128

- Eadie MJ. Unwanted effects of the anticonvulsant drugs. In: Tyrer $\mathbb{H}$, ed. The treatment of epillepsy. Lancaster: MTP, 19806:129-160

- Eisen SA, Miller DK, Woodward RS, Spitznagel E, Przybeck TR. The effect of prescribed daily dose frequency on patient medication compliance. Arch Intern Med 1990; 150:1881-1884.

- Engelsman M, Plesman P, Meijer JWA, Meinardi H. Single-dose escalation trial with loreclezole as comedication. A safety and pharmacokinetic study. Acta Neurol Scand 1990;82 (suppl 133):40

- Engberts DP. Overwegingen ten overwloede; het toestemmingsvereiste en het medisch experiment. In: Engberts DP, Bennebroek Gravenhorst J, Dupuis HM, Vermey P, Wintzen AR, eds. Informed consent: schijn en werkelijkheid. Boerhaave Commissie voor Postacademisch Onderwijs in de Geneeskunde, Boerhave cursus. Leiden: Rijksuniversiteit Leiden, 198:11-19 - Erwin VG, Dietrich RA. Inhibition of bovine brain aldehyde reductase by anticonvullsant compounds in vitro. Biochem Pharmacol 1973; 22:2615-2624

- Faigle JW, Feldmann KF. Carbamazepine - Biotransformation. In: Levy RH, Dreifuss FE, Matson RH, Meldrum BS, Penry JK, eds. Antiepileptic drugs. New York: Raven, 1989:491-504 - Fariello RG. Biochemical approaches to seizure mechanisms: the GABA and gltamate systems. 111. Porter RU, Morselli PL, eds. The Epilepsies. London: Butterworths, 1985:1-19

- Fariello R, Smith MC. Vatproate - Mechanisms of action. In Levy RH, Dreifuss FE, Mattson RH, Meldrum BS, Penty JK, eds. Antiepileptic drugs. New York: Raven, 1989:567-575

- Farmacotherapeutisch Kompas 1990 - 1991. Medisch farmaceutische voorlichting/uitgave van de centrale Medisch Pharmaceutische Commissie van de Ziekenhuisraad, 1982. Van der Kuy A. ed. Amstelween, 1990:68-83

- Farwell JR, Lee Y., Hirtz DO, Sulzbacher SI, Ellenberg JH, Nelson KB. Phenobarbital for rebrile seizures - effects on intelligence and on seizure recurrence. N Eng J Med 1990;322:364. 369

- Feldman RG, Pippinger CE. The relation of anticonvulsant drug lewels to complete seizure control. J Clin Pharmacol 1976; 16:51-59

- Forrari MD, Peeters AEJ, Han J, Roos RAC, Vermey P. De Wolff FA, Buruma OJS. Cylochrome P-450 and Parkinson's disease - Poor parahydroxylation of phenytoin. J Neurol Sci $1900: 96: 153-1.57$

- Ferrarese C, Appollonio 1. Frigo M, Piolti R, Tamma F. Frattola L. Distribution of a putative endogenous modulator of the GABAergic systen in human brain. Neurology 1989; 39:443-445 
- Ferrendelli JA. Phenytoin cyclic nucleotide regilation in the brain. Adv Neurol 1980; $27: 429$. 433

- Ferrendelli JA, Holland KD. Ethosuximide - Mechanisms of action In: Levy RH, Dreifuss FE, Mattson RH, Meldrum BS, Penry JK, eds. Antiepilleptic drugs. New York: Raven, 1989:653-661 - Fingl E General principles - Absorption, distribution, and elimination: practical pharmacokinetics. In: Woodbury DM, Penry IK, Schmidt D, eds. Antiepileptic drugs. New York: Raven, 1972: 7-21

- Forsgren L. Prospective incidence study and clinical characterization of seizures in newly referred adults. Epilepsia 1990; 31:292-301

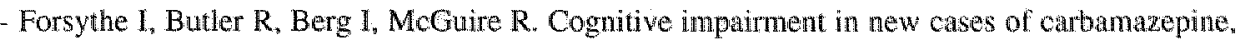
phenytoin and sodium valproate. Dev Med Child Neurol 1991; 33.524-534

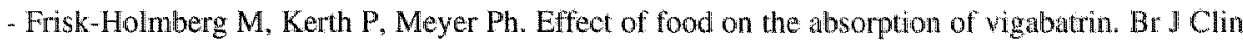
Pharmac 1989; 27 (suppl 1):23S-25S

- Fusco L, Iani C, Faedda MT, Manfredi M, Vigevano F, Ambrosetto G, Ciamatori C, Tassinari CA. Mesial frontal lobe epilepsy: a clinical entity not sufficiently described. J Epilepsy 1990; 3:123-135

- Gabor AJ. Lorazepam versus phenobarbital: candidates for drug of choice for treatment of status epilepticus. J Epilepsy 1990; 3:3-6

- Gale K. Progression and generalization of seizure discharge: anatomical and neurochemical substrates. Epilepsia 1988; 29 (suppl 2):S15-S34

- Gale K. GABA in epilepsy; the pharmacologic basis. Epilepsia 1989; 30 (suppl 3):SI-SII - Garaizar C, Prats JM. Tratamiento del sindrome de West con valproato a altas dosis. Epilepsia le Curso de formacion continuada, Accion medica. Barcelona, 1989:35-40

- Gellman RL, Kallianos JA, McNamara JO. Alpha-2 receptors mediate an endogenous noradrenergic suppression of kindling development. "Pharmacol Exp Ther 1987; 241:891-898 - Gillham RA, Williams N, Wiedmann KD, Butler E, Larkin JG, Brodie MJ. Cognutive function in adult epileptic patients established on anticonvulsant monotherapy. Epilepsy Res. 1990; 7:219. 225

- Gilbert JC, Wyllie MG. The effects of anticonvulsant ethosuximide on adenosine triphosphatase activities of synaptosomes prepared from rat cerebral cortex. Br J Pharmacol 1974:52:139P-140P - Glazko AJ. Phenytoin - Chemistry and methods of determination. In: Levy RH. Dreifuss FE, Matson RH, Meldrum BS, Penry JK, eds. Antiepileptic drugs. New York: $\mathbb{R}$ aven, 1989:159-176 - Godin Y, Heiner L. Mark $J$, Mandel P. Effects of di-ti-propylacetate, an anticonvulsan compound, on GABA metabolism. J Neurochem 1969 ; 16:869-873

- Godschalk M, Dzoljic MR, Bonta IL. Slow wawe sleep and a state resembling absence epilepsy induced in the rat by $\gamma$-hydroybutyrate. Eur J Phatrmacol 1977; 44:105-111

- Grace AA, Bunney BS. Paradoxal GABA excitation of nigral dopaminergic cells: indirect mediation through reticulata inhibitory neurons. Eur J Pharmacol 1979;59:211-218

- Graham DIM., Eadie J, Leadbetter BM, Metcalf R. Simultaneous determination of nafimidone [1-(2-naphthoy/methy\|)imidazole], a new anticonvulsant agent, and a major metabolite in plasma by high-performance liquid chromatography. J Chromatogr 1983;275:21 1-2.16

- Graham D. Neuropathology of vigabatrin. Br J Clin Pharmac 1989: 27 (supp 1):43S-45S

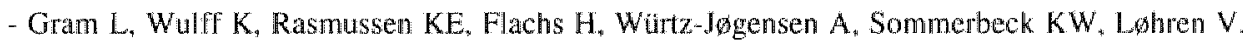
Valproate sodium; a controlled clinical trial including monitoring of drug levels. Epilepsia 1977 ; $18: 141-1.48$

- Gram L, Bentsen KD, Parnas J, Flachs $H$. Clinical trials in epilepsy: methods and results. In: 
Dam M, Gram L, Penry JK, eds. Advances in epileptology: XIth Epilepsy International Symposium. New York: Raven, 1981:105-112

- Gram L, Bentsen KD, Parnas J, Flachs H. Clinical trials in epilepsy: a review. Epilepsia 1982 ; 23:491-519

- Gram L. Experimental studies and controlled clinical testing of valproate and vigabatrin. Acta Neurol Scand 1988; 78:241-270

- Gram L, Larsson OM, Johnsen AH, Schousboe A. Effects of valproate, vigabatrin and aminooxyatetic acid on release of endogenous end exogenous GABA from cultured neurons. Epilepsy Res 1988; 2:87-95

- Gram L. Development trends in anti-epileptic treatment. 18th International Epilepsy Congress. Trileptal satellite symposium. New Delhi, 1989

- Gram I Kosterskov Jensen P. Carbamazepine - Toxicity. In: Levy RH, Dreifuss FE, Mattson RH, Meldrum BS, Penry JK, eds. Antiepileptic drugs. New York: Raven, 1989:555-565

- Gram L, Larsson OM, Johnson A, Schousboe A. Experimental studies of the influence of vigabatrin on the GABA system. Br J Clin Pharmac 1989a; 27 (suppl 1):13S-17S

- Gram L. Potentiall antiepileptic drugs, Lamotrigin. In: Levy RH, Dreifuss FE, Mattson RH, Meldrum BS, Penry JK, eds. Antiepileptic drugs. New York: Raven, 1989b:947-953

- Graves NM, Fuerst RH, Cloyd IC, Brundage RC, Welty TE, Leppik IE. Progabide-induced changes in carbamazepine metabolism. Epilepsia 1988; 29:775-780

- Guberman A, Couture M, Blaschuk K, Sherwin A. Add-on trial of clobazam in intractable adult epilepsy with plasma level correlations. Can J Neurol Sci 1990; 17:311-316

- Haefely WE. Biological basis of the therapeutic effects of benzodiazepines. In: Priest RG, Vianna Filho U, Amrein R, Skreta M, eds. Benzodiazepines, today and tomorrow. Lancaster: MTP, 1980:19\%46

- Haefely W. Benzodiazepines - Mechanisms of action. In: Levy RH, Dreifuss FE, Mattson RH, Meldrum BS, Penry JK, eds. Antiepileptic drugs. New York: Raven, 1989:721-734

- Haidukewych D, Rodin EA. Free (unbound) valproic acid in epilepsy patients medicated with coanticonvulsants: in vivo variable concentration and explanation for "nontherapeutic" plasma levels. In: Dam M, Gram L, Penry JK, eds. Advances in epileptology: XIIth Epilepsy International Symposium. New York: Raven, 1981:555-562

- Hajek KK, Cook NI., Novak RF. Mechanism ol inhibition of microsomal drug metabolism by imidazole. J Pharmacol Exp Ther 1982; 223:97=104

- Halonen T, Pitkanen A, Saano V, Riekkinem PJ. Effects of vigabatrin ( $\gamma$-winyl GABA) on neurotransmission-related animo acids and on GABA and benzodiazepine receptor binding in rats. Epilepsia 1991; 32:424:249

- Hartley R, Forsythe WI, Mclain B, Ng PC. Daily variations in steady-state plasma concentrations of carbamazepine and its metabolites in epileptic children. Clin Pharmacokinet $1991 ; 20 \div 237-244$

- Hauptmann A. Luminal bei Epilepsie. Münchener medizinische Wochenschrift 1912; 59: $1907-$ 1908

- Henriksen O. The place of combination therapy. In: Shorvon SD, Birdwood GFB, eds. Rational approaches to anticonvulsant drug therapy. Bern: Hans Huber Publishers, 1984:90-94

- Herranz JL, Arteaga R, Armijo JA. Side effects of sodium valproate in monotherapy controlled by plasmalevels: a study of 88 paediatric patients. Epilepsia $1982 ; 23: 203-214$

- Hoppener RJ, Kuyer A, Meijer JWA, Hulsman J. Correlation between dailly fluctuations of carbamazepine serum levels and intermittent side effects. Epilepsia 1980;21:341-350 
- Horn CS, Ater SB, Hurst DL. Carbamazepine-exacerbated epilepsy in children and adoleseents. Pediatr Neurol 1986;2:340 345

- Houtkoper MA, Van Oorschol CAEH, Rentmeester TW, Höppener PJEA, Onkelinx C. Doubleblind study of milacemide in hospitalized therapy-resistant patients with epilepsy. Epilepsia 1986 ; $27: 255-262$

- Hulsman JARJ, Meijers CAM, Höppener RJEA. The signifinance of the estimation of free concentration of drugs in patients on monotherapy of valproate or carbamazepine. In: Porter RJ, R.H.Porter, Ward Jr AA, Dam M, eds. Adwances in epileptology, XVth Epilepsy International Symposium. New York: Raven, 1984, 161-169

- Hulsman J, Rentmeester Th, Parijs JV, Klasterkov-Jensen P. Intraindividual comparison between conventional Tegretol USP and two controlled release Formulations, Tegretol CR Divitabs and Tegretol OROS. Epilepsia 1989; $30: 638$

- Hulsman J. Hyperammonemia and use of antiepileptic drugs including valproate. Epilepsia $1989 ; 30: 647-648$

- Ichikou N, leiri I, Higuchi S, Hirata K, Yamada H, Aoyama T. Analysis of the factors influencing antiepileptic drug concentrations - carbamazepine. Journal of Clinical Pharmacy and Therapeutics 1990; 15:337-349

- leiri 1, Higuchi S, Hirata $\mathrm{K}$, Yamada $\mathrm{H}_{0}$ Aoyama T. Analysis of the factors influencing antiepileptic drug concentrations - valproic acid. Journal of Clinical Pharmacy and Therapeutics $1990 ; 15: 351-363$

- Jackson JH. The Lumleian Lectures on convulsive seizures. Lancet 1890; 685-688

- Janz D. Die Epilepsien. Stutgart: Thieme, 1969

- Jeavons PM. Valproate non-dose-related side effects. Epilepsia 1984; 25 (suppl 1):S50-S55

- Jenkins SA, Edmonds Jr HL, Palohemo M, Zhang YP. Anticonvulsant testing of R 72063 in a rat model of postischaemic sound-induced convulsions. Janssen Research Foundation, Prechinical Report: R 72063/8 (N 62089), Beerse: December 1987

- Johannessen SI. Phenobarbital - Chemistry and methods of determination. In: Levy RH, Dreifuss FE, Mattson RH, Meldrum BS, Penry JK, eds. Antiepileptic drugs. New York: Raven, 1989:283-292

- Johnson AL. Statistical aspects of anticonvulsant trials. In: Shorvon SD. Birdwood GFB. eds. Rational approaches to anticonvulsant drug therapy. Bren: Hans Huber Publishers, 1984:13-21 - Jonkers JR, Merkus FWHM. "Patient compliance" of therapietrouw. In: Merkus FHMW, ed. Het voorschrijwen van geneesmiddelen. Utrecht: Bohn, Scheltema \& Holkema, 1978:228-237

- Kălviäinen R, Aikiä M. Saksa M, Sivenius J, Riekkinen PJ. Cognitive effects of vigabatrin monotherapy. Acta Neurol Scand 1990; 82 (suppl 133):13

- Kapetanovic IM, Kupferberg HJ. Nafimidone, an imidazole anticonvulsant and its metabolite as potent inhibitors of microsomal metabolism of phenytoin and carbamazepine. Drug Metab Dispos $1984 ; 12: 560-564$

- Kapetanovic IM, Kupferberg HJ. Jnhibition of microsomal phenytoin metabolism by nafimidone and related invidazoles. Drug Metab Dispos $1985 ; 13: 430-437$

- Kapetanovic IM. Drug metabolism and drug interactions. Acta Pharm Jugosl 1990; 40:351-362 - Kaplan SA. Phamacokinetics of the benzodiazepines. In: Priest RG, Vianna Filho U, Amrein R, Skreta M, eds. Benzodiazepines, today and tomorrow. Lancaster: MTP, 1980:47-60

- Kaplan SA, Jack ML. Metabolism of the benzodiazepines: pharmacokinetic and 
phamacodynamic considerations. In: Costa $\mathrm{E}_{i}$ ed. The benzodiazepines: from molecular biology to clinical practise. New York: Raven, 1983:173-200

- Kasteleijn-Wolst Trenite DGA. Photosensitivity in epilepsy. Utrecht: Rijksuniversiteit te Utrecht, 1989

- Keranen T, Riekkinen $\mathrm{PJ}$, Sillanpáa M. Incidence and prevalence of epilepsy in adults in eastem Finland Epilepsia 1989:30:413-421

- Kerr BM, Lewy RH. Carbamazepine - Carbamazepine epoxide. In: Levy RH, Dreifuss FE, Mattson RH, Meldrum BS, Penry JK, eds. Antiepileptic drags. New York: Raven, 1989:505-520

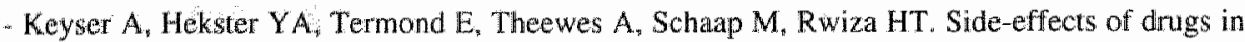
epileptic patients. Pharm WeekbI (Sci) 1990; 12:145-150

- Koella WP. Schmutz M. Preclinical development of new antiepileptic drugs - the present and the future. In: Dain M, Gram L, Penry JK, eds. Advances in epileptology: XIIth Epilepsy. International Sympositum. New York: Raven, 1981:7-12

- Koeppen D, Baruzzi A, Capozza M, Chauvel P, Courjon J, Favel P, Harmant J, Lorenz H, Oller FVL, Procaccianti G, Rucquoy-Ponsar M, Sallon C, Sideri G, Trotier S, Weber M, Wolf P. Clobazam in therapy-resistant patients with partial epilepsy: a double blind placebo-controlled crossover study. Epilepsia 1987; 28:495; 506

- Kotlarek F, Rübenstrunk U, Raemakers V, GroB-Selbeck G, Kellermann $K$, Rheingans $K$, Mortier W, Pothmann R, Von Bernuth $H$, Schauseil-Zipf $U$. A study of valproate in infants with West syndrome. In: Chadwick $D$, ed. Fourth International symposium on sodium valproate and epillepsy. London: Royal Society of medicine Services Ltd, 1989; 87-90

- Kraemer G, Hopf HC. Carbamazepin in der Neurologie. Stuttgart: Thieme, 1987

Kupferberg HJ, Swinyard EA, Gladding GD. ADD program of NINCDS: early pharmacological evaluation of new antiepileptic drugs. In: Dam M, Gram L, Penry JK, eds. Advances in epileptology: XIIth Epilepsy International Symposium. New York: Raven, 1981:13-18

- Kupferberg HJ. Antiepileptic drug development program: a cooperative effort of government and industry. Epilepsia 1989; 30 (suppl 1):\$51-\$56

- Kuschinsky G, Lüllmann H. Antiepileptika. In: Kurzes Lehrbuch der Pharmakologie. Stuttgart: Georg Thieme Verlag, 1970:162-166

- Kutt H. Mechanisms of action of antiepileptic drugs. In: Magnus O, Lorentz de Haas AM, eds. The epilepsies, Amsterdam: North-Holland Publishing Company, 1977:621-663 (Vinken PJ, Bruyn GW, eds. Handbook of clinicall neurology, vol 15)

- Kutt H., Phenytoin - relation of plasma concentration to seizure control. In: Woodbury DM, Penry JK. Pippinger CE, eds. Antiepilleptic Drugs. New York: Raven, 1982: 241-246

- Kutt H. Phenobarbital - Interactions with other drugs. In: Lewy RH, Dreifuss FE, Mattson RH, Meldrum BS, Penry JK, eds. Antiepileptic drugs. New York: Raven, 1989a:313-327

- Kutt H. Phenytoin - Interaction with other drugs. In: Lewy RH, Dreifuss FE, Mattson RH, Meldrum BS, Penry JK, eds. Antiepileptic drugs. New York: Raven, 19896:215-232

- Lacolle JY. Fertandes B, Eymard P. Profile of anticonvulsant activity of sodium valproate. Role of GABA. In: Meimardi H, Rowan AJ, eds. Advances in Epileptology. Amsterdam: Swets \& Zeitlinger, 1978:162-167

- Lander CM, Eadie MJ. Antiepileptic drug intake during pregnancy and malformed offspring. Epilepsy Res 1990; 7:77-82

- Lanoir J, Naquet R, Réquin S. Etude expérimentale comparative de l'action anticonvulsivante de la chlordiazépoxide (Librium) et du diazépan (Valium). Résultat préliminaires. Rev Neurol 1964:

$110: 22$ 
- Leppik IE. Introductory remarks and symposium overvew: Epilepsia 1989; 30 (suppl 1):S1-S2 - Levy RH, Shen DD. Valproate - Absorption, distribution, and excretion. In: Levy RH, Dreifuss FE, Mattson RH, Meldrum BS, Penry JK, eds. Antiepileptio drugs. New York: Raven, 1989 a: 583-599

- Levy RH. Unadkat JD. General principles - Drag absorption, distribution, and elimination. Ir: Levy RH, Dreifuss FE, Mattson RH, Meldrum BS, Penry JK, eds. Antiepileptic drugs New Xork: Raven, 1989b:1-22

- Li S-C, Schoenberg BS, Wang C.C, Cheng X-M; Zhou S-S, Bolis CL. Epidemiology of epilepsy in urban areas of the Peoples" Republic of China. Epilepsia 1985; 26:391-394

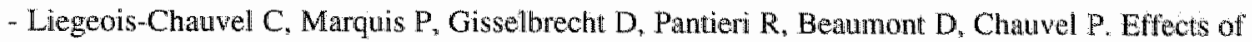
long term vigabatrin on somatosensory evoked potentials in epileptic patients. $\mathrm{Br} J$ Clin Pharmac 1989; 27 (suppl 1):69S-72S

- Liljequist S. The competitive NMDA receptor antagonist, CGP 39551 , inhibits ethanol withdrawal seizures. Eur J Pharmacol 1991; 192:197-198

- Lindhout D, Meinardi H. Gebruik van valproinezuur gedurende de zwangersehap: een indicatie voor prenataal onderzoek op spina bifida. Ned Tijdschr Geneesk 1984a; 128:2438-2440

- Lindhout D. Höppener RJEA, Meinardi $H$. Teratogenicity of antiepileptic drug combinations with special emphasis on epoxidation (of carbamazepine). Epilepsia $1984 b ; 25 ; 77-83$

- Lindhout D, Comel MC. Spectrum of neural tube defects in 26 cases prenatally exposed to antiepileptic drugs. Tenth Anniversary David W. Smith - Workshop on Malformations and Morphogenesis. Madrid, 1989

- Livingstone $\mathrm{JH}_{3}$ Beaumont D, Arzimanoglou A, Aicardi J. Vigabatrin in the treatment of epilepsy in children. Br J Clin Pharmac 1989;27 (suppl 1):109S-112S

- Loiseau P, Duche B. Carbamazepine - Clinical use. In: Levy RH, Dreifuss FE, Mattson RH, Meldrum BS, Penry JK, eds. Antiepileptic drugs. New York: Raven, 1989:533-554

- Loiseau J, Loiseau P, Guyot M, Duche B, Dartigues J-F. Aublet B. Survey of seizure disorders in the french southwest. I. Incidence of epileptic syndromes. Epilepsia 1990;31; 391-396

- Lombroso CT. Intermittent home treatment of status and clusters of seizures. Epilepsia 1989; 30 (suppl 2):S11-S14

- Luma D, Dulac O, Pajor $\mathbb{N}$, Beaumont D. Vigabatrin in the treatment of childhood epilepsies: a single-blind placebo-controlled study. Epilepsia 1989; 30:430.437

Macdonald RL, McLean MJ. Cellular bases of barbiturate and phenyloin anticonvulsant druy actions. Epilepsia 1982 (suppl 1); 23:\$7-\$18

- Macdonald RL. Antiepileptic drug actions. Epilepsia 1989a (suppl 3); 30:S19\%\$28

- Macdonald RL. Carbamazepine - Mechanisms of action. In: Levy RH, Dreifuss FE, Mattson RH, Meldrum BS, Penry JK, eds. Antiepileptic drugs. New York: Raven, 19896:447-455

- Marangos PJ, Weiss SRB, Montgomery P, Patel $\mathbb{J}$, Narang PK, Cappabianca AN, Post RM. Chronic carbamazepine treatment increases brain adenosine teceptors. Epilepsia 1985; 26:493. 498

- Marescaux C, Hirsch E, Gaultier C. Kurtz D. Carbamazepine (CBZ) and phenytoin (PHT) aggravate idiopathic generalized epilepsies of the child and adolescent. I8th International Epilepsy Congress, New Delhi, 1989

- Marrosu F. Argiolas A, Carcangiu P, Giagheddu M, Fraata W. Neonatal monosodium glutamatie abolishes corticotropin-releasing factor-induced epileptogenic activity in rats. Epilepsia 1990; 31:708-712

- Matthes A. Epilepsie. Stuttgart: Thieme, 1977 
- Mattson RU, Cramer JA. Phenobarbital - Toxicity. In: Levy RH, Dreifuss FE, Mattson RH, Meldrun BS, Penry IK, eds. Antiepileptic drugs. New York: Raven, 1989a 341-355

- Mattson RH, Cramer JA. Valproate - Interactions with other drugs. In: Levy RH. Dreifuss FE, Mattson RH, Meldrum BS, Penry JK, eds. Antiepileptic drugs. New York: Raven, 1989b:621-632 - MoDonald JW, Garofalo EA, Hood T, Sackellares JC, Gilman S. McKeever PE, Troncoso JC, Johnston MV. Altered excttatory and inhibitory amino acid receptor binding in hippocampus of patients with temporal lobe epilepsy. Ann Neurol 1991; 29:529-541

- McNamara JO. Development of new pharmacological agents for epilepsy; lessons from the kindling model. Epilepsia 1989; 30 (suppl 3):S13-\$18

- Meijer IWA. Knowledge, attitude and practice in antiepileptic drug monitoring. Nijmegen: Katholieke Universiteit, 1991

- Meinardi H. Clinical trials of anti-epileptic drugs. Psychiat Neurol Neurochir 1971; 74:141-151

- Meinardi H. Anti-epileptica. In: Lammers W, Nelemans FA, Bouwman ThJ, Van Noordwijk

J, Offerhaus L, Rosinga WM, Vermeulen A, eds. Algemene farmacotherapie. Alphen aan den Rijn: Stafleu, 1980:359-377

- Meinardi H, Binnie CD, Goedhart D, Meijer JWA. Developments in pharmacotherapy of epilepsy. In: Meinardi $H_{*}$ Porter RJ, eds. Epilepsy: an update on research and therapy. New York: Liss, 1983:249-265

- Meinardi H. Anti-epileptica. Ned Tijdschr Geneeskd 1987; 131:1292-1296

- Meldrum B, Braestrup C. GABA and the anticonvulsant action of benzodiazepines and related

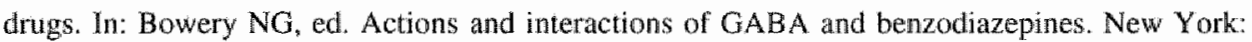
Raven, 1984:133-153

- Meldrum BS. GABAergic mechanisms in the pathogenesis and treatment of epilepsy. Br J Clin Pharmac 1989; 27 (suppl 1):3S-11S

_ Meldrum BS. Anatomy, physiology, and pathology of epilepsy. In: Epilepsy, a Lancet Review. London, 1990:11-14

- Melis MR, Gale K. Effect of dopamine agonists on GABA turn wer in the superior colliculus: evidence that nigrotectal GABAergic projections are under the influence of dopaminergic transmission. J Pharmacol Exp Ther 1983; 226:425-431

- Merritt HH, Putnam TJ. A new series of anticonvulsant drugs tested by experiments on animals. Arch Neurol Psychiat 1938; 38:1003-1015

- Meunier H, Carraz G, Meunier Y, Eymard P, Aimard M. Proprietés pharmacodynamiques de l'acíde n-dipropylacétique. Thérapie 1963; 18:435-438

- Michelucci R. Tassinari CA. Response to vigabatrin in relation to seizure type. Br J Clin Pharmac 1989; 27 (suppl 1):119S-124S

- Mireles R, Leppik IE. Valproate and clonazepam comedication in patients with intractable epilepsy. Epilepsia 1985; 26:122.126

- Miraa W. Credeur J, Penry JK. Results of antiepileptic drug reduction in institutionalized epileptic patients with multiple handicaps. Epilepsia 1989:30:663

- Mitchell WG, Crawford TO. Lorazepam is the treatment of choice for status epilepticus. J Epillepsy $1990 ; 3: 7-10$

- Molhler H, Richards JG. Benzodiazepine receptors in the central nervous system. In: Costa E, ed. The benzodiazepines" from molecular biology to clinical practise. New York: Raven, 1983:93116

- Morselli PL, Lloyd KG. Mechanisms of action of antiepileptic drugs. In: Porter RJ, Morselli PL, eds. The Epilepsies. London: Butterworths, 1985:40-81

- Morselli PL. Carbamazepine - Absorption, distribution and excretion. In: Levy RH, Dreifuss FE, 
Mattson RH, Meldrum BS, Penry JK, eds. Antiepileptic drugs. New York: Raven, 1989:473-490 - Murphy JM, Mashman J, Miller JD, Bell JB. Suppression of carbamazepine-induced rash wh prednisone. Neurology 1991; 41:144-145

- Newmark ME. Elective, rapid changes of antiepileptic drug (AED) therapy: an acceptable alternative to phased, slow withdrawal. American Academy of Neurology proceedings. Neurology 1990; 40 (suppl 1):188

- Nicoll RA, Eccles JC, Oshima T, Rubia F. Prolongation of hippocampal inhibitory postsynaptic potentials by barbiturates. Nature 1975;258:265-267

- Nies AS. Principles of therapeutics. In: Goodman Gilman A, Rall TW, Nies AS, Taylor P. Goodman and Gilman's the pharmacological basis of therapeutics. New York: Pergamon, 1990:62-83

- Nuwer MR, Browne TR, Dodson WE, Dreifuss FE, Engel Jr I, Leppik IE, Mattson RH, Penty JK, Treiman DM, Wilder BJ. Generic substitutions for antiepileptic drugs. Neurology 1990; 40:1647 1651

- Obeid T, Panayiotopoulos. Clonazepam in juvenille myoclonic epilepsy. Epilepsia 1989: 30:603606

- Osorio $\mathbb{1}$, Reed RC. Treatment of refractory generalized tonic-clonic status epilepticus with phenobarbital anesthesia after high-dose phenytoin. Epilepsia 1989;30:464-471

- Overweg I, Binnie CD, Meijer JWA, Meinardi H, Nuijten STM, Schmaltz S, Wauquier A. Double-blind placebo-controlled trial of flunarizine as add-on therapy in epilepsy. Epilepsia 1984; 25:217-222

- Overweg J. Withdrawal of antiepileptic drugs in seizure-nfee adult patients. Ansterdam: Universiteit van Amsterdam, 1985

- Overweg J, De Beukelaar F. Single-dose efficacy evaluation of loreclezole in patients with photosensitive epilepsy. Epilepsy Res 1990;6:227-233

- Painter MJ. Phenobarbital - Clinical Use. In: Levy RH, Dreifuss FE, Mattson RH, Meldrum BS, Penry JK, eds. Antiepileptic drugs. New York: Raven, 1989:329.340

- Palmer ED, Cawthorne MA. The effects of 1-alkylimidazoles on hepatic drug-metabolizing enzyme activity. Xenobiotica 1974:4:209-217

- Parsonage M. Epilepsy in adults. In: Tyrer JH,ed. The treatment of epilepsy. Lancaster: MTP. 1980: 95-128

- Pedersen B. Anderson G. Treatment of epilepsy in old age. In: Chadwick D, ed. Fourth Intemational Symposium on sodium valproate. London: Royal Society of Medicine, 1990:130132

- Perucca E, Richens A. Anticonvulsant drug interactions. In: Tyrer JH, ed. The treatment of epilepsy. Lancaster. MTP 1980: 95-128

- Pisani F, Caputo M, Fazio A, Oteri G, Russo M, Spina E, Perucca E, Bertilisson L Interaction of carbamazepine-10,11-epoxide, an active metabolite of carbamazepine, with valproate: a pharmacokinetic study. Epillepsia 1990; 31:339-342

- Pitlick WH, Levy RH. In: Levy RH, Dreifuss FE, Mattson RH, Meldrum BS. Penry JK, eds. Antiepileptic drugs. New York: Rawen, 1989:521-531

- Polet ThW. Bijdrage tot de symptomatologie der epileptische bewustzijnsstoomissen. Utrecht: Rijks-universiteit te Utrecht, 1911

- Polkey CE. Neurosurgery. In: Laidlaw J, Richens A, Oxley J, eds A texubook of epilepsy. 


\section{Edinburgh: Churchill Livingstone, $1988 ; 484-510$}

- Porter RJ. General principles - Clinical efficacy and use of antiepleptic drugs. In: Woodbury DM. Perny JK, Pippinger CE, eds. Antiepileptic drugs. New York: Raven, 1982: 167-175

- Ponter RI. The empirical use of models in the development of antiepileptic drugs. In: Porter RI. Morselli PL, eds. The Epilepsies. London: Butterworths, 1985a:82-93

- Ponter RJ. Toxicity of antiepileptic drugs. In: Dam M, Gran L, Pedersen B. Orum H. Modern approach to antiepileptic drug treatment. Kobenhavn: The Danish Epilepsy Society, 19856:45-54 - Porter $\mathbb{R} J$. Mechanisms of action of new antiepileptic drugs. Epilepsia 1989a, 30 (suppl 3):\$29534

- Porter RJ. General principles - How to use antiepileptic drugs. In: Levy RH, Dreifuss FE, Mattson RH, Meldrum BS, Penry JK, eds. Antiepileptic drugs. New York: Raven, 1989 b: 117-131 - Porrer RJ. New antiepifeptic agents; strategies for drug development. In Epilepsy, a Lancet Review. London, 1990:25-27

- Prescott LF. Safery of the benzodiazepines. In: Costa $\mathbb{E}$, ed. The benzodiazepines: from molecular bjology to clinical practise. New York: Raven, 1983:253-265

- Prichard JW. Antiepileptic drugs - phenobarbital: proposed mechanisms of antiepileptic action. In: Glaser GH, Penry JK, Woodbury DM, eds. Antiepileptic drugs." mechanisms of action. New York: Raven, $1980: 553-562$

- Prichard JW. Phenobarbital - Mechanisms of action. In: Woodbury DM, Penry IK, Pippinger CE, eds. Antiepileptic drugs. New York: Raven, 1982: 365-376

- Prichard JW, Ransom BR. Phenobarbital - Mechanisms of action. In: Levy RH. Dreifuss FE, Mattson RH, Meldrum BS, Penry JK, eds. Antiepileptic drugs. New York: Raven, 1989:267-282 - Pryka RD, Rodvold KA, Erclman SM. An updated comparison of dirug dosing methods - Part I: phenytoin. Clin Pharmacokinet 1991; 20:209-217

- Pullar T, Birtwell AJ, Wiles PG, Hay A, Feely MP. Use of a pharmacologic indicator to compare compliance with tablets prescribed to be taken once, twice, or three times daily. Clin Pharmacol Ther $1988 ; 44: 540-545$

- Racine RJ, Ivy GO, Milgram NW. Kindling: clinical relevance and anatomical substrate. In: Bolwig TG, Trimble MR, eds. The clinical relevance of kindling. Chichester: Wiley, 1989:15-34 - Rall TW, Schleifer LS. Drugs effective in the therapy of the epilepsies. In: Goodman Gilman A. Rall TW, Nies AS. Taylor P. Goodman and Gilman's the pharmacological basis of therapeutics. New York: Pergamon, 1990:436 462

- Ramsay RE, Wilder JM, Pellock JM, Dreifuss FE, Mattson RH. Smith DB, Peny JK, Sunder TR, Abmann PA, Spitz MC, Murphy JV, Leroy RF, Morris DD, Hansen RG, Pierce MW. Successful conversion from polytherapy to Depakote monotherapy in patients with primary generalized tonic-clonic seizures. Epilepsia 1989; 30:662-663

- Rumsay RE. Pharmacokinetica and clinical use of parenteral phenytoin, phenobarbital, and paraldehyde. Epilepsia 1989; 30 (suppl 2):S1\%S3

- Rentmeester T, Hulsman J. Long acting valproate versus conventional formulation of VPA. An open substitution trial, 4th British. Danish Dutch Epillepsy Congress, Heemstede, 1988

Rentmeester T, Hulsman J. Long acting valproate versus conventional formulation of valproate. In: Chadwick D, ed. Fourth International Symposium on sodium valproate. London: Royal Society of Medicithe 1989, 185-19!

- Rentmeester TW. Hulsman JARJ, Doelman JC. Carbanazepine, merk- en generiek preparaat. Pharm Weekbl 1990; 125:1108-1110

- Rentmeester TW. Carbamazepine, merk- en generiek preparat (III) Pharm Weekbl 1991; 


\section{6:100-101}

- Reynolds EH, Shorwon SD. Monothicapy or polytherapy for epilepsy? In: Canger $\mathbf{R}$, Angeleri fit Penry $J \mathrm{~K}$, eds. Advances in epileptology: XIh International Symposium. New York: Raven, $1980: 407-413$

- Reynolds EH, Shorvon SD. Monotherapy or polytherapy for epilepsy? Epilepsia 1981:22:1-10 - Reynolds EH. Phenytoin - Toxicity. In: Levy RH, Dreifuss FE, Mattson RH, Meldrum BS, Penry JK, eds. Antiepileptic drugs. New York: Rawen, 1989:241-255

- Rjchens A. Interactions with antiepileptic drugs. Drugs 1977; 13:266-275

- Ried S, Schmidt D. Withdrawal seizures after discontinuation of vigabatrin. Satellite symposium Latest advances in vigabatrin research, 18 th International Epilepsy Congress, New Dellhi, 1989 - Rimmer EM, Richens A. Clinical pharmacology and medical treatment. In: Laidlaw J, Richens

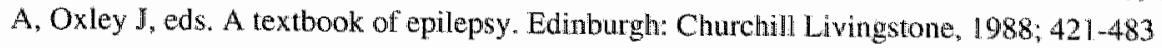

- Rimmer EM, Richens A. Interaction between vigabatrin and phenytoin. Br J Clin Pharmac 1989. 27 (suppl 1):27S-33S

- Ring HA, Heller AJ, Farr IN, Reynolds EH. Vigabauin: rational treatment for chronic epilepsy. I Neurol Neurosurg Psychiatry 1990; 53:1051-1055

- Robb P. Epidemiology of epilepsy. In: Magnus O, Lorentz de Haas AM, eds. The epilepsies, Amsterdam: North-Holland Publishing Company, 1977:491-497 (Vinken PJ. Bruyn GW, ads. Handbook of clinical neurology, vol 15)

- Robinson M, Richens. A. Behavioural disturbances related to vigabatrin treatment. Acta Neurol Scand 1990; 82 (suppl 133):38

- Rodin EA, Haidukewych D. Fluctuations in free valproic acid (VPA) levels of epileptic patients. In: Dam M, Gram L, Penry JK, eds. Advances in epileptology: XIIth Epilepsy International Symposium. New York: Raven, 1981:541-548

- Rosa $\mathbb{F W}$. Spina bifida in infants of women treated with carbamazepine during pregnancy. $N$ Engl J Med 1.991; 324:674-677

- Rosenberg HC, Tietz EI, Chau TH. Tolerance to anticonvulsant effects of diazepam, clonazepam, and clobazam in amygdala-kindled rats. Epillepsia 1989; 30:276-285

- Rowan AJ, Overweg J, Meijer JWA. Monodose therapy with valproic acid: 24-hour telemetric EEG and serum level studies. In: Dam M, Gram L, Penry JK, eds. Advances in epileptology: XIIh Epilepsy International Symposium. New York: Raven, 1981:533-539

- Rust RS, Dodson WE. Phenobarbital - Absorption, distribution, and excretion. In: Levy RH, Dreifuss FE, Mattson RH, Meldrum BS, Penry JK, eds. Antiepilleptic drugs. New York: Raven, 1989:293-304

- Rutgers MJ. Geneeskundige en matschappelijke aspecten wan de zorg voor epilepsiepatiènten in Nederland. Rotterdam: Erasmus Universiteit, 1984

- Sabers A. Cognitive function and drug treatment. In: Sillanpala M, Johannessen S1, Blennow G, Dam M. eds. Paediatric epilepsy. Petersfield: Wrightson Biomedical Publishing 1990:225-232 - Salvato JA. Torres C. Wat K. Reduction of antiepileptic drug therapy in mentally retarded epileptic patients. Epilepsia 1.989:30:663

- Sander JWAS, Hart YM, Trimble MR, Shorvon SD. Psychosis associated with the prescription of vigabatrin. Acta Neurol Scand 1990; 82 (suppl 133 ):37

- Sander JWAS, Hart YM, Trimble MR. Shorwon SD. Vigabatrin and psychosis. I Neurol Neurosurg Psychiatry 1991:54:435-439

- Sato S. Benzodiazepines - Clonazepam. In: Levy RH, Dreifuss FE, Matson RH, Meldrum BS, Penry JK, eds. Antiepileptic drugs. New York: Raven, 1989:765-784 


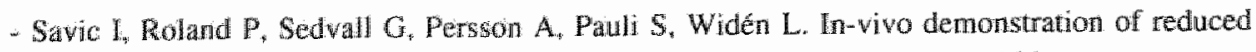
benzodiazepine receptor binding in thuman epileptic foci. Lancet 1988; 2:863-866

- Savic I, Widen L, Thorell JO. Blomqvist G, Ericson K. Rolland P. Cortical benzodiazepine receptor binding in patients with generalized and partial epilepsy. Epilepsia 1990; 31:724-730 - Sawaya MCB, Horton RW, Meldrum BS. Effects of anticonvulsant drugs on the cerebral enzymes metabolizing GABA. Epilepsia 1975; 16:649-655

- Schauf CL, Davis FA, Marder J. Effects of carbamazepine on the ionic conductances of myxicola giant arons. J Pharmacol Exp Ther 1974; 189:538-543

- Schechter PJ. Clinical pharmacology of vigabatrin. Br J Clin Phamac 1989; 27 (suppl 1):19S$22 \mathrm{~s}$

- Scheffner D, Konig St, Rauterberg-Ruland I, Kochen W, Hofmann WJ, Unkelbach St. Fatal liver failure in 16 children with valproate treatment. Epilepsia $1988 ; 29: 530-542$

- Scheunemann W. Brom - eine therapeutische Chance bei fruhkindlicher Grand-mal-Epilepsie. Jahrestagung der Deutschen Sektion der Internationalen Liga gegen Epilepsie, Mannheim, 1983 - Schmidt D. Die Behandiung der Epilepsie mit Hilfe der Blutspiegelbestimmung. Nervenarzt $1977 ; 48: 183-196$

- Schnidt D. Adverse effects of antiepileptic drugs. New York: Raven, 1982 a

- Schmidt D. Two antiepileptic drugs for intractable epillepsy with complex-partial seizures. I Neurol Neurosurg Psychiatry 1982b; 45:1119-1124

- Schmidt D. Discontinuation of antiepileptic drugs. In: Porter RJ, Morselli PL, eds. The Epilepsies. London: Butterworths, 1985:227-241

- Schmidt D, Rohde M, Wolf P, Roeder-Wanner U. Clobazam for refractory focal epilepsy. A controlled trial. Arch Neurol $1986 ; 43: 824-826$

- Schmidt D. Berzodiazepines - Diazepam. In: Levy RH, Dreifuss FE, Mattson RH, Meldrum BS, Penry IK, eds. Antiepileptic drugs. New York: Raven, 1989:735-764

- Schobben AFAM. Pharmacokinetics and therapeutics in epilepsy. Nijmegen: Katholieke Universiteit, 1979

- Schoonbrood-Lenssen AMJ, Starrenburg RThM. Tien kleine negertjes... - Mogelijkheden voor klinisch geneesmiddelenonderzoek bij geestelijk gehandicapten. Nederlands Tijdschrift voor Zwakzinnigenzorg 1990; 16:92-98

- Schwarz JR. The mode of action of phenobarbital on the excitable membrane of the node of Ranvier. Eur o Pharmacol 1979; 56:51-60

- Scollo-Lavizzarri G. Therapy of partial epilepsy. In: Chadwick D, ed. Fourth International Symposium on sodium valproate. London: Royal Society of Medicine, 1990: 102-105

- Scoville B, White BG, Cereghino JJ, Porter RJ. Suitability and efficacy of crossover drug trial designs in epilepsy. In: Dam M, Gram L. Penry JK. eds. Advances in epileptology: XIIth Epilepsy International Symposium. New York: Raven, 1981:113-122

- Semmes RLO, Shen DD. Comparative pharmacodynamics and brain distribution of E- $\Delta 2$. valproate and valproate in rats. Epilepsia 1991: 32:323-24\|

- Shaner DM, McCurdy SA. Herring MO Gabor AJ. Treatment of status epilepticus: a prospective comparison of diazepam and phenytoin versus phenobarbital and optional phenytoin. Neurology 1988; 38:202-207

- Sherwin A. Quesney F, Gauther S, Olivier A, Robitaile Y, McQuaid P, Harvey C, Van Gelder N. Enzyme changes in actively spiking areas of thuman epileptic cerebral cortex. Neurology 1984 ; $34: 927.933$

- Sluerwin AL. Ethosuximide - Clinical use. In: Levy RH, Dreifuss FE, Matson RH, Meldrum BS, Penry JK, eds. Antiepileptic drugs. New York: Raven, 1989:685-698 
Shields WD, Saslow E. Myoclonic, atonic and absence seizures following institution of carbamazepine therapy in children. Neurology 1983:33:1487-1489

- Shofer JB, Tenkin NR. Comparison of alternatiwe outcome measures for antiepileptic drug trials. Arch Neurol 1986; 43:877-881

- Shorvon SD. The role of single drug and combination drug therapy in the treatment of epilepsy. In: Dam M, Gram L, Pedersen B, Orun H, eds. Modern approach to antiepileptic drug treatment. Københaun: The Danish Epillepsy Society, 1985:45-54

- Shorvon SD. Benzodiazepines - Clobazam: In: Lewy RH, Dreifuss FE, Matson RH. Meidrum BS, Penry JK, eds. Antiepileptic drugs. New York: Raven, 1989:821-840

- Siegel S, Castellan Jr NJ. Nonparametric statistics for the behavioral sciences. New York: McGraw-Hill, 1988

- Sieghart W. Heterogeneity of benzodiazepine receptors. In: Sauvanet IP. Luanger SZ, Morselli PL, eds. Imidazopyridines in sleep disorders. New York: Raven, 1988:39-45

- Sillanpäa M. Carbamazepine. Pharmacology and clinical uses. Acta Neurol Scand 1981: 64 (suppl 88):11

- Sitsen JMA. Drug-drug interactions: CNS-drugs - possibilities and limitations. In: Breimer DD, Merkus FWHM, eds. Drug-drug interactions and food-drug interactions. Boerhaave Commissie voor Postacademisch Onderwijs in de Geneeskunde, Boerhamve cursus. Leiden: Rijksuniversiteit Leiden 1991:101-115

- Skerritt JH, Davies LP, Johnston GAR. Interaction of the anticonvulsant carbamazepine with adenosine receptors. 1: Neurochemical studies. Epilepsia $1983 a ; 24: 634-642$

- Skerritt JH, Johnston GAR, Chow SC. Interaction of the anticonvulsant carbamazepine with adenosine receptors. 2: Pharmacological studies. Epilepsia 1983b; 24:643-650

- Smith MC, Bleck TP. Convulsive disorders: toxicity of anticonvulsants. Clin Neuropharm 1991; $14: 97 / 115$

- Snead III OC. Gamma hydroxybutyrate in the monkey - I. Electroencephalographic, behavioral, and pharmacokinetic studies. Neurology 1978a; 28:636-642

- Snead III OC. Gamma hydroxybutynate in the monkey - II. Effect of chronic oral anticonvulsant drugs. Neurology 1978b; 28:643-648

- Snead III OC. Gamma hydroxybutyrate in the monkey - III. Effect of intravenous anticonvulsant drugs. Neurology $1978 c ; 28: 1173-1178$

- Snead TII OC. The ontogeny of GABAergic enhancement of the gamma-hydroxybutyrate model of generalized absence seizures. Eplepsia 1990;31:363-368

- Sohn RS, Ferrendelli JA. Anticonvulsant drug mechanisms " phenytoin, phenobarbital, and ethosuximide and calcium flux in isolated presynaptic endings. Arch Neurol 1976;33:626-629

- Spiller HA, Krenzelok EP. Cookson E. Carbamazepine overdose: a prospective study of serum levels and toxicity. Clin Toxicol 1990; 28:445-458

- Steinhoff B, Kruse R. Bromide therapy of tonic-clonic epiltepsies; a clinical study. Ejpilepsia $1990 ; 31: 225-226$

- Sternbach LH. The discovery of CNS active $\|$ 4-benzodiazepines. In: Costa $\mathrm{E}$, ed. The benzodiazepines. New York: Raven, 1983:1-6

- Stricker BHCh. Post-marketing surveillance: begrip, definitie, systemen en methoden. In:Breimer DD. De Fijter JW, Merkus FWHM, Overbosch D, eds. Post-marketing surveillance van werkingen en bijwerkingen van geneesmiddelen na 1992. Boerhave Commissie voor Postacademisch Onderwijs in de Geneeskunde, Boerhaave cursus. Leiden: Rijksuniversiteit Leiden $\rrbracket 989: 35-47$

- Subirana A, Oller-Daurella L. Laggrevation de certains cas d"epilepsie soit par les traitements 
anti-pileptiques, soit par les therapeotiques couramment associés. Rev Newrol $1963 ; 110 ; 21-22$ - Sullivan Jr JB, Rumack BH, Peterson RG. Acute carbamazepine toxicity resulting from werdose. Neurology, 1981; 31:621-624

- Swartz BE, Delgado-Esscueta AV, Maldonado HM. A stereoencephalographic study of ictal propagation producing anisocoria, auras of fear, and complex partial seizures of temporal lobe origin. J Epilepsy $1990 ; 3: 149 \% 156$

- Theisohn $\mathrm{M}_{\text {, }}$ Demant $L$, Sigmund $\mathrm{M}$. Verânderte Metabolisierungswege van Carbamazepin fach chronischer Gabe bei geunden Probanden. In: Remschmidt $H$, Rentz $R$, Jungmann $J$, eds. Epilepsie 1981 - Verlauf und Prognose, neuropsychologische und psychologische Aspekte. Stutgart: Thieme, 1983:213-220

- Thomas $J_{*}$ Jones L, Van der Vliet V, Edson A, Price S, Betts T, Harding G. Assessment of neurotoxicity of vigabatrin by serial evoked potentials and psychometric testing in patients with refractory epillepsy. Acta Neurol Scand 1990;82 (suppl J 33):38

- Tomson T, Almkvist O, Nilsson BY, Swensson J-O, Bertilsson L. Carbamazepine-10, 11 -epoxide in epilepsy. Arch Neurol 1990; 47:888 892

- Treiman DM, Ben-Menachem E, Barber KO, Chelberg R. Inhibition of carbamazepine and phenytoin metabolism by nafimidone, a new antiepileptic drug. Neurology 1984; 34 (suppl 1): 213

- Treiman DM, Wilenski AJ, Ben-Menachem E, Ojemann L, Yerby M, Barber KO, McCormick $\mathrm{KB}$, Cereghino JJ, White BG, Swisher $\mathrm{K}$. Elficacy of nafimidone in the treatment of intractable partial seizures: report of a two-center pilot study. Epilepsia 1985; 26:607-61 I

- Treiman DM. Pharmacokinetics and clinical use of benzodiazepines in the management of status epilepticus. Epilepsia 1989; 30 (suppl 2):\$4-\$10

- Trimble MR. Anticonvulsant drugs and cognitive funetion: a review of the literature. Epilepsia 1987; 28 (suppl 3):537-S45

- Trimble MR, Cull C. Children of school age: the influance of antiepileptic drugs on behaviour and intellect. Epilepsia $1988 ; 29$ (suppl 3):S15-\$19

- Trimble MR. Antiepileptic drugs, cognitive function, and behaviour in children: evidence from recent studies. Epilepsia 1990; 13 (suppl 4):530-534

- Trother S, Chodkiewicz JP. Beamont D, Chauvel P. Hauw JJ, Escourolle R. Neuropathological examination of surgical samples of brain tissue of a patient treated with vigabatrin. 17th Epilepsy Intemationat Congress. Focus on vigabatrin. Jerusalem, 1987

- Trottier S, Sazdovitch V, Chauvel P, Beaumont D. Hauw JJ. Neuropathological study of brain tissue in six patients treated with long-tern vigabatrin. 18th Epilepsy International Congress. Latest advances in vigabatrin research. New Delhi, 1989

- Van der Laan JW, De Boer Th, Brumvels J. Di-n-propylacetate and GABA degradation. Preforential inhibition of succinic semialdehyde dehydrogenase and indirect inhibition of GABAtransuminase. J Neurochem 1979; 32:1769-1780

- Var der Pol MC. Hadders-Algra M. Huisjes HJ, Touwen BCL. Antiepileptic medication in pregnancy: Late effects on the children's central nervous system development. Am J Obstet Gynecol $199 !: 164: 121-128$

- Van de Velde V, De Beukelaar F, Van Rooy P, Van Peer A, Woestenborghs R, Vanden Bussche G. Heykants J. Oral pharmacokinetics and bioavailability of $R 72063$ in healthy volunteers. Janssen Research Foundation Climical Report: R 72063/2 (N 56899), Beerse: September 1987 - Van de Velde V, Van Peer A, De Benkelaar F. Van Rooy P, Woestenborghs R, Heykants J, 
Vanden Bussche G. Pharmacokinetics of R 72063 after single oral adminstration of a 100 -mg dose to a healthy volunteer. Janssen Research Foundation Clinical Report: R $72063 / 4$ (N 59724$)$. Beerse: January 1988 a

- Van de Velde V, De Beukelaar F, Van Rooy P, Van Peer A, Woestenborghs R, Heykunts J, Vanden Bussche G. Multiple dose pharmacokinetics of R 72063 in healthy volunteers after oral administration of $50 \mathrm{mg}$ o.d. for 7 days, followed by $10 \mathrm{mg}$ o.d. for 14 days. Manssen Resench Foundation Clinical Report: R $72063 / 5$ (N 62347), Beerse: Apriil $1988 b$

- Van Gool JD, Theunissen PMVM. Foetale echografie en neurale-buisdefecten; morfologische diagnose zonder klinische zekerheid. Ned Tijdschr Geneesk 1990; 50:2447-2448

- Van Heycop ten Ham MW. Epilepsie. Leiden: Stafleu, 1974:67

- Van Peer A, De Beukelaar F, Gasparini R, Woestenborghs $\mathbb{R}$, Pauwels C. Heykants *, Vanden Bussche G., Pharmacokinetics after oral administration of $R 57720$ : a review of available single and repeated doses of $10 \mathrm{mg}, 40 \mathrm{mg}$ and $80 \mathrm{mg}$ in healthy volunteers. Janssen Research Products Information Service R 57 720/4 (N 40617), Beerse: November 1984

- Van Wieringen A, Vrijland CM. Ethosuximide intoxication caused by interaction with isoniazid. Neurology 1983; 33:1227-1228

- Vermeij P. "Dat is niet zo gemakkelijk ait te leggen". In: Engberts DP, Bennebroek Gravenhorst J. Dupuis HM, Vermey P, Wintzen AR, eds. Informed consent: schijn en werkelijkheid. Boerhaave Commissie voor Postacademisch Onderwijs in de Geneeskunde, Boerhaswe cursus. Leiden: Rijksuniversiteit Leiden, 1988:39-51

- Vining EPG. Cognitive dysfunction associated with antiepileptic drug therapy. Epilepsia 1987 ; 28 (suppl 2):S18-S22

- Wagner ML, Graves NM, Marienau K, Holmes GB, Remmel RP, Leppik IE. Discontinuation of phenytoin and carbamazepine in patients recieving felbamate. Epilepsia 1991; 32:398-406

- Wamsley JK, Golden JS, Yamamura HI, Barnett A. Autoradiographic demonstration of the selectivity of two 1-N-trifluoroethyl benzodiazepines for the $\mathrm{BZD}$-1 receptors in the rat brain. Pharmacol Biochem Behav 1985; 23:973-978

- Watanabe K. The localization-related epilepsies: some problems with subclassification, Jpn J Psychiatry Neurol $1989 ; 43: 471-475$

- Waqquier A, Ashton D, Clincke G, Fransen J. R 51 107: a new broad spectrum anticonwulsant. Janssen Research Products Information Service R 51 107/1 (N 31841). Beerse: May 1983

- Waquier A, Fransen J, Melis W, Ashton D, Gillardin JM, Van Clemen G. R 72 063: a broad spectrum anticonvulsant. Janssen Research Information Service Prechinical Research Report: R 72 063/2 (N 56239), Beerse: February 1988 a

- Wauquier A, Melis W, Wan den Broeck W. R 72063 increases the threshold to generalized EEG seizures in dogs induced by metrazol infusion. Janssen Research Foundation Preclinical report: $R$ $72063 / 6$ (N 59941), Beerse: March 1988b

- Wauquier A. Smeyers F. Melis F, Woestenborghs R. R 72063 protects against metrazolinduced convulsions in dogs. Janssen Research Foundation Preclinical Report: R 72 063/7 (N 59942). Beerse: March 1988 c

- Wauquier A, Fransen J, Melis W, Ashton D, Gillardin J-M, Lewi PJ, Van Clemen G, Vaught J, Janssen PAJ. Loreclezole ( $R 72$ 063): an anticonvulsant chemically unrelated to protolype antiepilleptic drugs. Drug Development Research 1990; 19:375-392

- White $\mathrm{BG}_{n}$ Cereghino JJ. Clinical trial design for the efficacy evaluation of antiepileptic therapies. In: Dam M, Gram L. Penry JK. eds. Advances in epileptology: XIIth Epilepsy International Symposium. New York: Raven, 1981:129-134 